Computational study

of interfaces and

edges of 2D makerials

Mojtaba Farmanbar 


\section{Computational study of interfaces and edges of 2D materials}

Mojtaba Farmanbar 


\section{Composition of graduation committee:}

Chairman and secretary:

Supervisor:

Co-supervisor:

Members:
Prof. dr. ir. H. Hilgenkamp (University of Twente)

Prof. dr. P. J. Kelly (University of Twente)

Dr. G. Brocks (University of Twente)

Prof. dr. F. Peeters (University of Antwerpen)

Prof. dr. ir. H. S. J. van der Zant (Delft University of Technology)

Prof. dr. J.-S. Caux (University of Amsterdam)

Prof. dr. W. J. Briels (University of Twente)

Prof. dr. ir. G. Koster (University of Twente)
MESA+

INSTITUTE FOR NANOTECHNOLOGY

\section{UNIVERSITY OF TWENTE.}

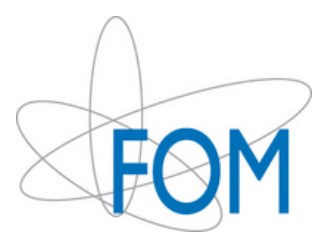

The work described in this thesis was carried out in the computational materials science group, MESA+ Institute for Nanotechnology, University of Twente, the Netherlands.

This work is part of the research programme of the Foundation for Fundamental Research on Matter (FOM), which is part of the Netherlands Organisation for Scientific Research (NWO). The use of supercomputer facilities was sponsored by the Physical Sciences Division (EW) of NWO.

Computational study of interfaces and edges of 2D materials

Mojtaba Farmanbar

PhD thesis University of Twente, Enschede

ISBN: 978-90-365-4125-1

DOI: $10.3990 / 1.9789036541251$

Copyright (CM. Farmanbar, 2016

Published by: M. Farmanbar

Cover Design: Sadaf Nadimi, www.sadafnadimi.com

Printed by: Gildeprint- Enschede 


\title{
COMPUTATIONAL STUDY OF INTERFACES AND EDGES OF 2D MATERIALS
}

\author{
DISSERTATION \\ to obtain \\ the degree of doctor at the University of Twente, \\ on the authority of the rector magnificus, \\ Prof. dr. H. Brinksma, \\ on account of the decision of the graduation committee, \\ to be publicly defended \\ on Friday 13 May 2016 at 16.45 \\ by \\ Mojtaba Farmanbar Gelepordsari \\ born on $16^{\text {th }}$ of December, 1983 \\ in Tehran, Iran.
}


This dissertation has been approved by:

Prof. dr. P. J. Kelly (promotor)

Dr. G. Brocks (assistant promotor) 
This work is dedicated to my father 



\section{Contents}

1 Introduction 1

1.1 Two-dimensional materials . . . . . . . . . . . . . . . 1

1.1.1 Monatomic 2D materials . . . . . . . . . . . . . . 2

1.1.2 Diatomic 2D materials . . . . . . . . . . . . . 3

1.2 Interfaces between $2 \mathrm{D}$ materials and metal contacts $\ldots \ldots \ldots$

1.3 1D edge states in 2D materials . . . . . . . . . . . . . 7

1.4 Computational Methods . . . . . . . . . . . . . . . . . 9

1.4.1 Density Functional Theory . . . . . . . . . . . . . . 9

1.4.2 Exchange and correlation functionals . . . . . . . . . . 10

2 A first-principles study of van der Waals interactions and lattice mismatch $\begin{array}{ll}\text { at } \mathrm{MoS}_{2} / \text { metal interfaces } & 15\end{array}$

2.1 Introduction . . . . . . . . . . . . . . . . . 15

2.2 Calculations ......................... 17

2.2.1 Computational Methods . . . . . . . . . . . . . . . 17

2.2.2 Comparison of DFT Functionals . . . . . . . . . . . . 20

2.2 .3 Lattice Mismatch . . . . . . . . . . . . . . . . . . 22

2.3 Results ........................ 26

2.3.1 Metal/MoS $\mathrm{MoS}_{2}$ interaction ................... 26

2.3.2 Interface Potential Step and Schottky Barrier . . . . . . . . . . . . . 29

$2.3 .3 \quad \mathrm{MoS}_{2} / \mathrm{Ti}(0001) \ldots \ldots \ldots \ldots \ldots$

2.4 Summary and Conclusions . . . . . . . . . . . . 35

3 Controlling the Schottky barrier at $\mathrm{MoS}_{2} \mid$ metal contacts by inserting a BN monolayer 39

3.1 Introduction. . . . . . . . . . . . . . . . . . 39

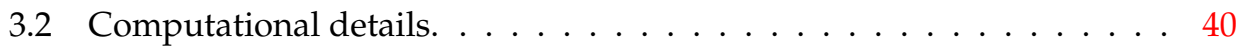

$3.3 \mathrm{MoS}_{2} \mid$ metal interfaces. . . . . . . . . . . . . . . . . 42

$3.4 \mathrm{MoS}_{2} \mid h$-BN|metal interfaces. . . . . . . . . . . . . . . 45

3.5 Conclusions. . . . . . . . . . . . . . . . . 47

4 Ohmic contacts to 2D semiconductors through van der Waals bonding 49

4.1 Introduction . . . . . . . . . . . . . . . . . . 49

4.2 Results and Discussion . . . . . . . . . . . . . . . 51

4.2.1 Van der Waals bonded contacts . . . . . . . . . . . . . 51 
4.2 .2 High electron affinity oxide layers ............ 55

4.2.3 Metallic $\mathrm{M}^{\prime} \mathrm{X}_{2}^{\prime}$ buffer layers . . . . . . . . . . . . . . . . 58

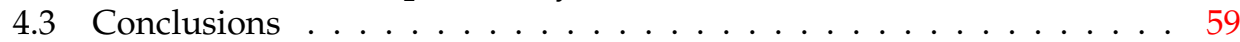

4.4 Computational section . . . . . . . . . . . . . . 60

5 Green's function approach to edge states in transition metal dichalcogenides 63

5.1 Introduction . . . . . . . . . . . . . . . . 63

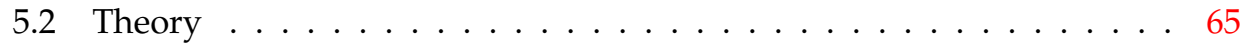

5.2 .1 Green's functions . . . . . . . . . . . . . . . . 65

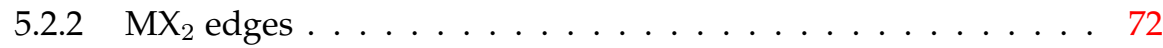

5.2 .3 Tight-binding models . . . . . . . . . . . . . 75

5.3 Results .......................... 77

5.3.1 Three-band model . . . . . . . . . . . . . . . 77

5.3.2 Eleven-band tight-binding model ............. 82

5.4 Summary and Conclusions . . . . . . . . . . . . 88

6 One-dimensional edge states of 2D transition metal dichalcogenide nanorib$\begin{array}{ll}\text { bons } & 91\end{array}$

6.1 Introduction . . . . . . . . . . . . . . . . . 91

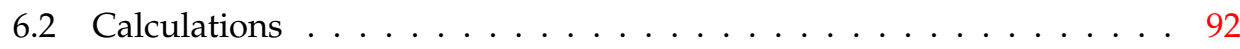

6.2 .1 Edge structures . . . . . . . . . . . . . . . 93

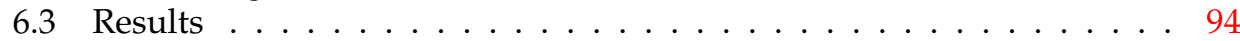

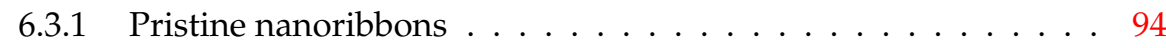

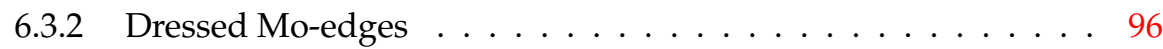

6.3 .3 Reconstructed S-edges . . . . . . . . . . . . . . . . . 98

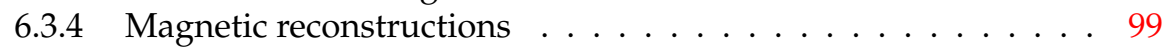

6.3 .5 Other $\mathrm{MX}_{2}$ compounds ................... 101

$\begin{array}{ll}\text { A Tight-binding model } & 105\end{array}$

$\begin{array}{ll}\text { Tight-binding model } & 105\end{array}$

A.1 Three-band tight-binding model . . . . . . . . . . . . . . . 105

A.2 Eleven-band tight-binding model . . . . . . . . . . . . . . . . . 106

A.2.1 Even states . . . . . . . . . . . . . . . . . . . . . . . . . . . . . . . . . . . . . .

A.2.2 Odd states . . . . . . . . . . . . . . . . . 108

$\begin{array}{ll}\text { References } & 111\end{array}$

$\begin{array}{ll}\text { Summary } & 129\end{array}$

$\begin{array}{ll}\text { Samenvatting } & 131\end{array}$

$\begin{array}{ll}\text { Acknowledgement } & 133\end{array}$

$\begin{array}{ll}\text { Publications } & 135\end{array}$ 


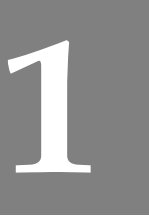

\section{Introduction}

\subsection{Two-dimensional materials}

In future there will be an increasing demand for foldable, bendable, and rollable devices that are wearable as easy as clothes or accessories [1,2]. Flexible devices will have the advantages of lightness, higher space efficiency, and improved comfort. The development of flexible devices requires breakthroughs in materials with desirable electronic and optical properties.

The discovery of graphene as a two-dimensional (2D) material has sparked considerable scientific interest because of its unique properties and potential applications in electronic devices [3, 4]. Graphene has excellent conducting and mechanical properties and shows a good adhesion to organic materials, which promises to boost the field of flexible organic electronics: organic field-effect transistors (OFETs), organic light-emitting diodes (OLEDs), and organic photovoltaic cells (OPVs) [1]. Graphene's unique band structure, the linear dispersion at the conical points in particular, gives rise to novel phenomena such as a room-temperature quantum Hall effect, and has opened up a new field of "Fermi Dirac" physics [5].

The quest for graphene analogues has resulted in the exploration of a variety of other 2D materials, such as the transition metal dichalcogenides (TMDs) [6], hexagonal boron nitride $(h$-BN) [4], and 2D elemental allotropes collectively termed Xenes [7]. The latter include silicene [8, 9], germanene [10] and phosphorene [11]. Materials as the TMDs, h-BN, or phosphorus, form naturally layered crystals with strong covalent bonding between the atoms in one layer, and much weaker van der Waals bonding between the layers. This type of bonding facilitates the isolation of atomically thin 2D sheets that have no dangling surface bonds, which makes them chemically stable under ambient conditions. Such 2D materials promise superior intralayer transport of fundamental excitations (charge, heat, spin and light) [12]. This thesis focuses on TMD layers. 
a) Graphene

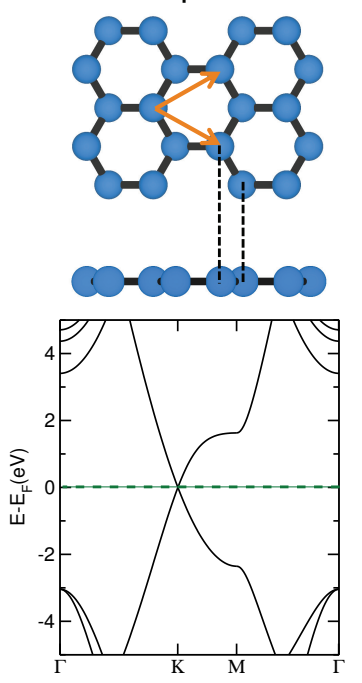

b) Germanene
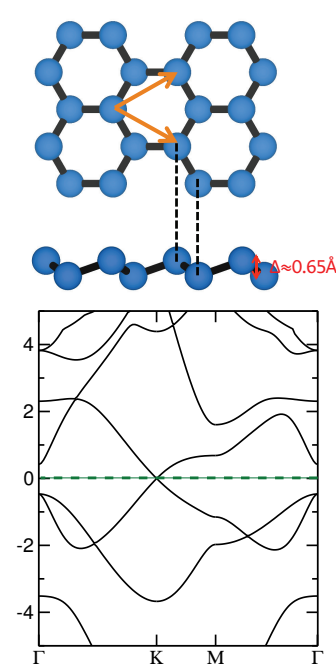

c) Black Phosphorus
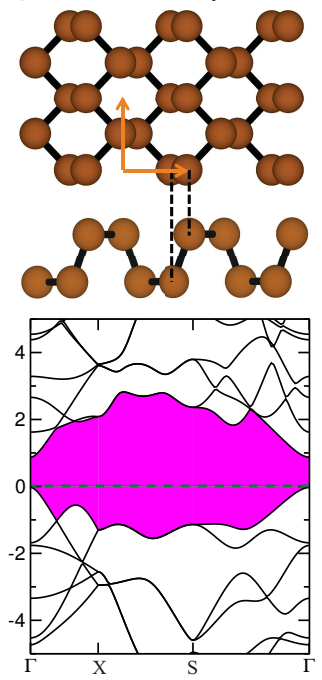

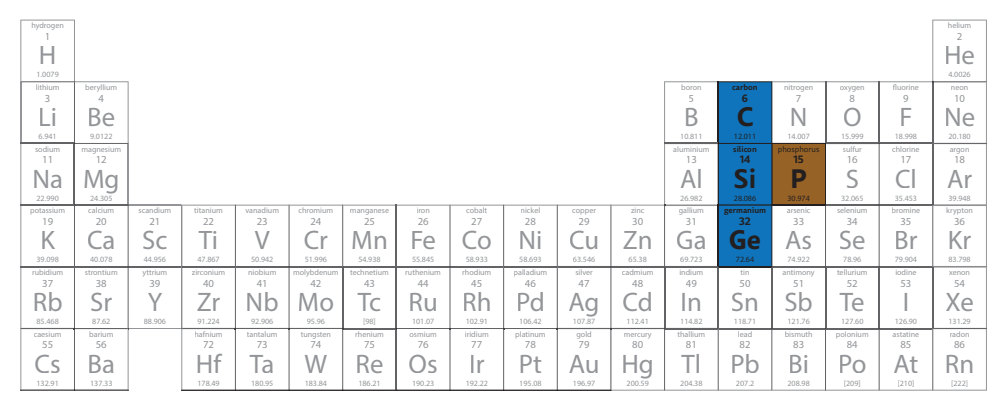

Figure 1.1: a) Top and side views of a layer of graphene, b) germanene, and c) black phosphorus, along with their corresponding band structures and positions in the periodic table.

\subsubsection{Monatomic 2D materials}

Graphene is a 2D atomic layer of $s p^{2}$-hybridized carbon. Its extended honeycomb network (Fig. 1.1) is the basic building block of other important allotropes; graphene can be stacked to form 3D graphite, rolled into 1D nanotubes, and wrapped into 0D fullerenes [4]. Long-range $\pi$-conjugation gives graphene extraordinary electronic, mechanical, and thermal properties, which has become an exciting area of research for experimentalists and theorists alike. The experimental isolation of single-layer graphene with a high carrier mobility has given access to a wealth of interesting physics, for instance, an ambipolar field effect, a quantum Hall effect at room temperature, and the first detection of single molecule adsorption [1,5]. Future applications envision the use of graphene in high-speed logic devices, thermally and electrically conductive reinforced composites, sensors, and transparent electrodes for displays and solar cells [1]. 

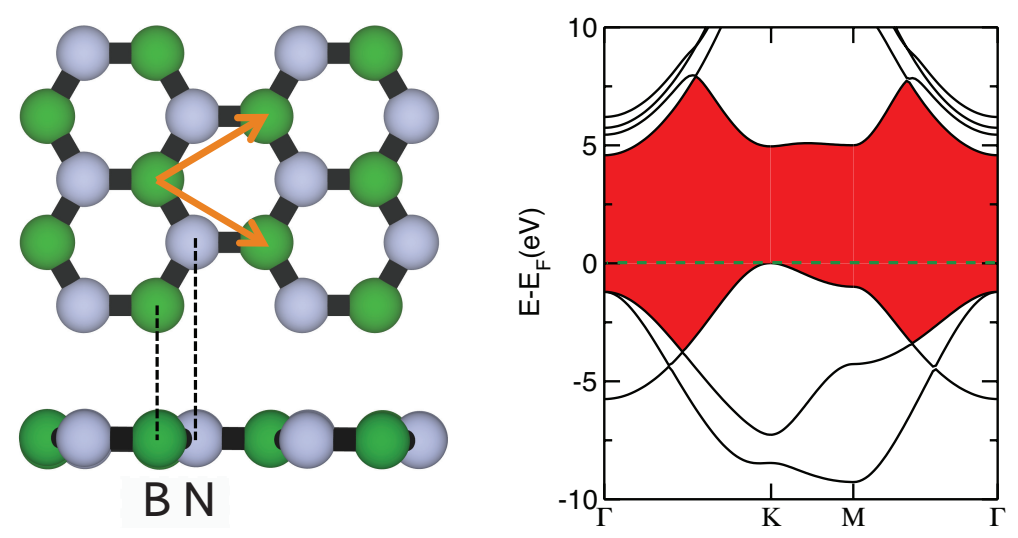

Figure 1.2: (left) Top and side views of $h$-BN and (right) the band structure of $h$-BN.

Silicon, germanium, and tin come from the same chemical family as carbon. Unlike carbon, the elements silicon, germanium, and tin do not naturally form 2D layers. The key element for carbon forming planar 2D honeycomb structures lies in the relative stability of $s p^{2}$ hybridization. In other group IV elements $s p^{3}$ hybridization is more stable, which leads to 3D diamond-like structures, for instance. Nevertheless there is a large interest in making artificial 2D structures from group IV elements, called silicene, germanene, and stanene. Calculations predict that such structures prefer to have a corrugated hexagonal lattice (Fig. 1.1) [13]. Germanene, silicene and graphene share several very peculiar and interesting electronic properties. The electronic levels of these materials near the $\mathrm{K}$ and $\mathrm{K}^{\prime}$ points of the Brillouin zone are described by a linear dispersion relation, and the electrons in the corresponding states behave as relativistic massless particles.

Black phosphorus (black P), which is the most stable allotrope of elemental phosphorus, has recently been rediscovered as a $2 \mathrm{D}$ layered material $[14,15]$. The atomic layers in black $\mathrm{P}$ have a puckered geometry (Fig. 1.1). Phosphorus has one electron per atom more than the group IV elements, which allows for a closed-shell configuration to be formed within a $s p^{3}$-like hybridized 2D lattice of hexagons in chair conformations, as in saturated cyclic hydrocarbons. Black $\mathrm{P}$ is a semiconductor with a direct electronic band gap between 0.3 and $2 \mathrm{eV}$, depending on the number of phosphorus layers [16]. Because of its semiconducting properties, a high carrier mobility, and anisotropic in-plane properties, black $\mathrm{P}$ is promising for novel applications in electronics and photonics [17].

\subsubsection{Diatomic 2D materials}

An atomic layer of hexagonal Boron nitride ( $h$ - $\mathrm{BN})$ has a similar planar hexagonal lattice structure as graphene. A $h$-BN monolayer is a $s p^{2}$-bonded honeycomb lattice, where each hexagon is composed of alternating boron and nitrogen atoms (Figure 1.2). In contrast to graphene, $h$ - $\mathrm{BN}$ is an insulator with a large band gap of approxi- 


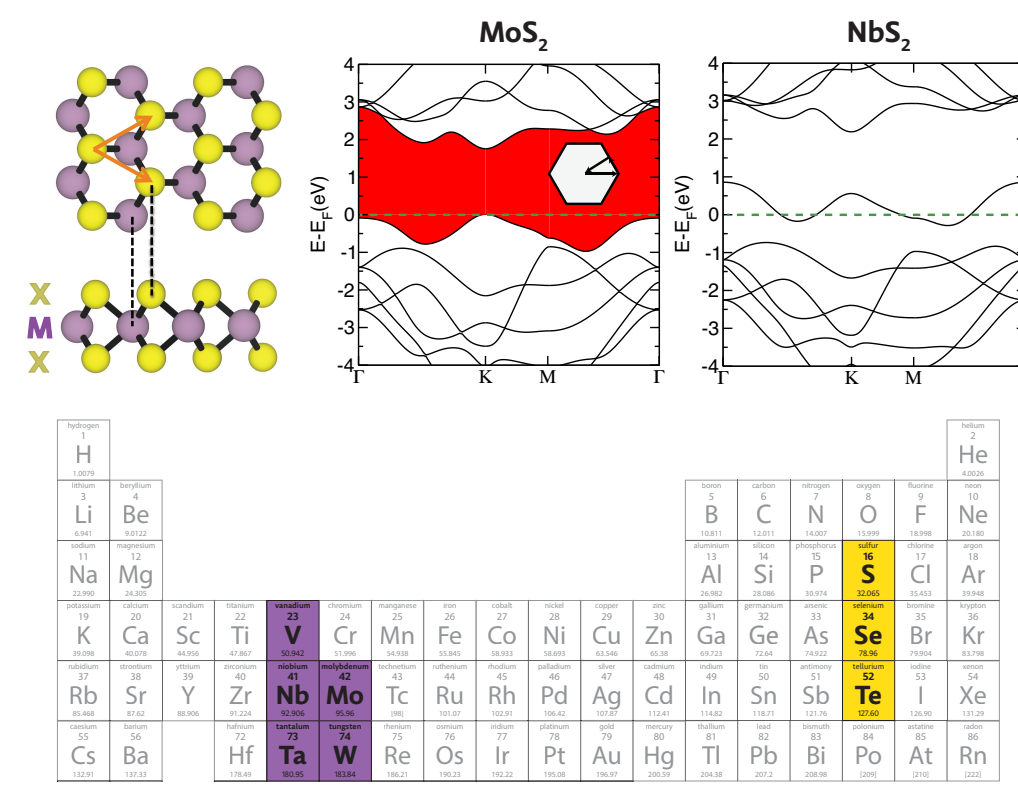

Figure 1.3: (top left) Top and side views of a $\mathrm{MX}_{2}$ monolayer; (top middle and right) band structures of a semiconducting $\mathrm{MX}_{2}\left(\mathrm{MoS}_{2}\right)$ and a metallic $\mathrm{MX}_{2}\left(\mathrm{NbS}_{2}\right)$ monolayer. The green dashed lines indicate the postions of the Fermi levels; (bottom) some of the elements forming stable $\mathrm{MX}_{2}$ layers.

mately $5.9 \mathrm{eV}[4,18]$. Because of its good electrical insulating qualities, bulk $h$-BN has been applied as a charge leakage barrier layer in electronic equipment [4]. The use of $h$-BN thin films as dielectric layers to gate graphene and as an inert flat substrate for graphene transistors has been shown to significantly improve device performance [19]. $h$-BN also shows far ultraviolet light emission, which is attributed to a direct band gap (Fig. 1.2) [20].

Transition metal dichalcogenides (TMDs) $\mathrm{MX}_{2}$ form an interesting classes of materials. Depending on the metal (M) species they display a wide range of physical properties such as semiconductivity, half-metallic magnetism, superconductivity, or charge density waves $[12,21]$. Bulk TMDs have applications in areas such as lubrication, catalysis, photovoltaics, supercapacitors, and rechargeable battery systems [22]. $\mathrm{MoS}_{2}$ is the prototypical TMD material. Bulk $\mathrm{MoS}_{2}$ is used for dry lubrication, as a catalyst for removing sulfur compounds from oil, and for hydrogen evolution in electrolysis [23]. Because of its absorption in the solar spectral region, bulk $\mathrm{MoS}_{2}$ has also attracted interest for its use in photovoltaic and photocatalytic materials $[22,24]$. Interestingly, whereas bulk $\mathrm{MoS}_{2}$ is a semiconductor with an indirect band gap of $1.29 \mathrm{eV}$, a single layer of $\mathrm{MoS}_{2}$ has a direct band gap of $1.86 \mathrm{eV}[24,25]$.

A monolayer of a transition metal dichalcogenide $\mathrm{MX}_{2}$ consists of a layer of metal atoms sandwiched between two layers of chalcogen atoms (Fig. 1.3). Two dominant structures are found, which differ in coordination of the transition metal by the 

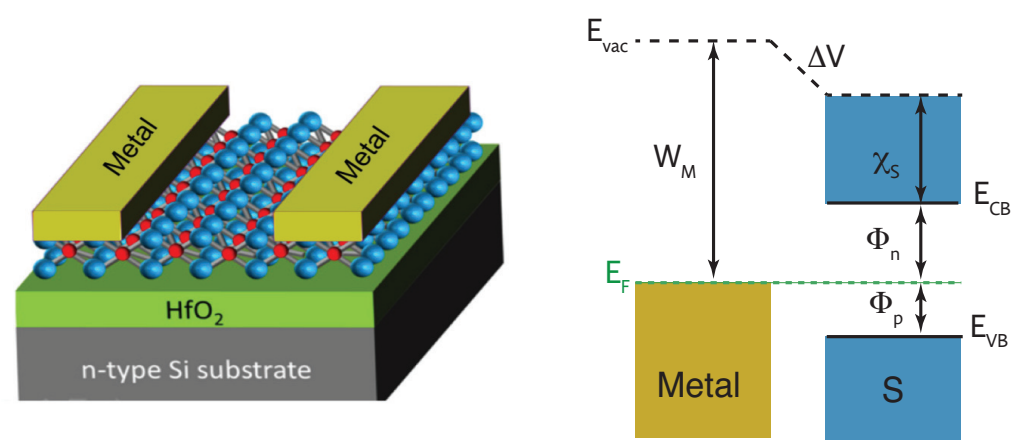

Figure 1.4: (Left) Sketch of a typical FET geometry; (right) sketch of the energy levels at a MS interface. The work function $W_{M}$ of a clean metal is changed by the formation of an interface potential step $\Delta V$, resulting from the interaction between the metal and the semiconductor; $\chi_{S}$ is the electron affinity of the semiconductor; $\Phi_{n}$ and $\Phi_{p}$ are the SBHs for electrons and holes, respectively.

chalcogen atoms [21]. In the $2 \mathrm{H}-\mathrm{MX}_{2}$ stucture, each $\mathrm{M}$ is coordinated by six $\mathrm{X}$ atoms in a trigonal prism with $D_{3 h}$ point group symmetry. From the top the resulting structure then looks like a honeycomb structure similar to $h$ - $\mathrm{BN}$. In the $1 \mathrm{~T}-\mathrm{MX}_{2}$ structures each $\mathrm{M}$ is coordinated by six $\mathrm{X}$ atoms in a octahedron with a $C_{3 v}$ symmetry. Depending on the coordination geometry and the oxidation state of the metal atoms, $\mathrm{MX}_{2}$ compounds can be semiconducting (e.g., $2 \mathrm{H}$ with $\mathrm{M}=\mathrm{Mo}, \mathrm{W}$ ) or metallic (e.g., $2 \mathrm{H}$ with $\mathrm{M}=\mathrm{Nb}$, Ta, or $1 \mathrm{~T}$ with $\mathrm{M}=\mathrm{Mo}$ ). This thesis concerns $2 \mathrm{H}-\mathrm{MX}_{2}$ layers.

\subsection{Interfaces between 2D materials and metal contacts}

It is technologically challenging to obtain defect-free 2D materials that can be used in devices. However, the properties of a device are not only determined by the quality of its components. In a 2D device geometry interfaces between the 2D layers, the substrate, and the electrodes, play a prominent role (Fig. 1.4) [26], and it is a significant challenge is to provide optimum interfaces. Metal-semiconductor (MS) contacts play a key role in electronic and photonic devices, as they markedly influence the transport behavior of charge carriers. A MS contact typically results in the formation of a Schottky barrier at the MS interface, where the Schottky-barrier height (SBH) is a measure of the mismatch of the energy levels for electrons or holes between metal and semiconductor at the MS interface (Fig. 1.4). The SBH controls electronic transport across a MS interface and is therefore of vital importance to the successful operation of any semiconductor device [27, 28, 29].

The electronic states responsible for electrical conduction in the semiconductor depend on the doping of the semiconductor. For $n$-type semiconductors, the electrons near its conduction band minimum are primarily responsible for electrical conduction, and for $p$-type semiconductors, holes near the valence band maximum carry most of the current. Because of the presence of a fundamental transport gap, 
the lowest-lying states for an $n$-type semiconductor that can communicate with electrons in the metal, are at an energy $\Phi_{n}$ above the Fermi level (Fig. 1.4). For electronic transport across the MS interface, this energy offset is the $n$-type SBH. Similarly, $\Phi_{p}$ is the SBH for transport of holes across the MS interface.

The Schottky-Mott limit (SBL) gives the $n$-type SBH between a metal with a work function $W_{M}$ and a semiconductor with an electron affinity $\chi_{S}$ as $[30,31]$

$$
\Phi_{n}=W_{M}-\chi_{S} .
$$

For a fixed semiconductor it has been found experimentally that when varying the metal contact, by and large, metals with larger work functions give larger SBHs than those with lower work functions, as in the Schottky-Mott limit. However, whereas Eq. 1.1 predicts a slope $S=d \Phi_{n} / d W_{M}=1$, experimentally it is found that $S$ is often very much smaller than 1 . For particular semiconductors, one even finds $S \approx 0$. The phrase "Fermi-level pinning" is used to describe the insensitivity of the SBH to the metal work function in those cases [32]. In practice, the characterization of SBHs by a single slope $S$ tends to be misleading, as the relation between $\Phi_{n}$ and $W_{M}$ is generally not linear, and depends on the details of the MS interface structure [28, 30, 33].

The reason that the SBL fails is obvious: the charge distribution at real MS interfaces is significantly different from a simple superposition of the charge distributions of the clean metal and semiconductor surfaces. The metal and semiconductor chemically interact at the interface, and the newly formed chemical bonds significantly modify the charge distribution at the interface. One can attribute the net change in the potential energy $\Delta V$ as a result of charge rearrangement at the interface to the formation of an interface dipole layer, and write (Fig. 1.4)

$$
\Phi_{n}=W_{M}-\chi_{S}-\Delta V,
$$

The interface potential step $\Delta V$ depends on the chemistry of the metal and semiconductor surfaces interacting at the interface $[28,30,34]$. There is no particular reason why $\Delta V$ should be a simple (linear) function of $W_{M}$, and in general it is not.

Some limiting cases can be identified, however. Surfaces of semiconductors such as $\mathrm{Si}$ have a significant density of surface states with energies in the band gap, often resulting from "dangling bonds" on the surface atoms [35, 36]. Upon binding the semiconductor to a metal, such surface states can be broadened into resonances, which fill the band gap if the broadening is sufficiently large. If the resulting density of states (DoS) of these so-called metal-induced gap states (MIGS) at the interface is sufficiently large, it pins the Fermi level, and the SBH is almost independent of the metal species. In other words $\Delta V \approx E_{\mathrm{MIGS}}-W_{M}$, with $E_{\mathrm{MIGS}}$ the pinning level (typically close to the local charge neutrality level of the semiconductor surface $[37,38,39])$. If the DoS of the MIGS is not large enough, but is approximately constant for energies in the band gap, then $\Delta V$ is a linear function of $W_{M}$, and $\Phi_{n}$ can be characterized by a single slope $S$. 
In the absence of a strong chemical interaction at the MS interface, one may expect the MIGS model to break down. If the interaction between metal and semiconductor is weak, one might guess that $\Delta V$ is small, and that the SBH obeys the SBL. This is in fact not true; even for weak van der Waals MS interactions sizable interface potential steps, $\Delta V \approx 1 \mathrm{eV}$, are found. Calculations trace this effect to a charge rearrangment at the MS interface, caused by Pauli exchange repulsion between the electrons of the metal and of the semiconductor surface [40]. The resulting potential step $\Delta V$ has no simple relation to the metal work function, and the SBL does not generally hold. However, for a weak MS interaction with a fixed semiconductor, $\Delta V$ can be modeled approximately as a monotonic function of a single parameter: the equilibrium distance $\left(d_{e q}\right)$ between the metal and semiconductor surfaces at the interface. Metals for which $d_{e q}$ is similar, give a similar $\Delta V$, and the corresponding SBHs then obey a modified SBL with a constant offset $\Delta V$.

Naturally occuring 2D materials, such as the TMDs, have no dangling bonds at their surface and no surface states. Hence the MIGS approach to SBHs is not likely to be valid. The Pauli repulsion model may not work either, because many metals form chalcogenide compounds, and the interaction between a TMD layer and a metal substrate is not necessarily weak.

In chapter 2 the interface properties of $\mathrm{TMD} /$ metal contacts are studied, using the prototype TMD, $\mathrm{MoS}_{2}$, and a range of metal substrates with different work functions and reactivities. The interaction between a $\mathrm{MoS}_{2}$ layer and a metal substrate ranges from strong chemisorption on Ti to weak physisorption on Au. The SBHs for the physisorbed cases obey approximately a modified SBL, whereas chemisorption tends to lead to Fermi level pinning, as shown in chapter 3. The Fermi level can be unpinned, and the SBL can be restored by inserting an inert 2D layer (such as $h-\mathrm{BN}$ ), between $\mathrm{MoS}_{2}$ and the metal substrate. This allows for obtaining a zero SBH between $\mathrm{MoS}_{2}$ and a well-chosen metal. As shown in chapter 4, the idea of inserting an inert 2D layer (such as $\mathrm{NbS}_{2}$ ) also works for designing contacts with zero SBHs to $\mathrm{MX}_{2}$ layers for holes.

\subsection{D edge states in 2D materials}

Whereas 2D materials such as the TMDs have no surface states, finite 2D samples have edges. An interesting feature of finite systems is the possibility of edge states. In particular, edge states with energies in the band gap of a semiconducting TMD are intriguing. As such states cannot exist in the interior the TMD, they must be localized at the edges. Edge states are localized at the material-vacuum boundary and decay exponentially away from it. They are realizations of one-dimensional (1D) electronic systems, and are subjected to the electronic effects induced by electronelectron correlation that are typical of 1D systems.

TMD edges show a surprisingly rich structure of edge states. They are not topologically protected, but they are not easily destroyed either. Even in undoped form many TMD edges give 1D metallic structures. Moreover, as the TMD lattice lacks 


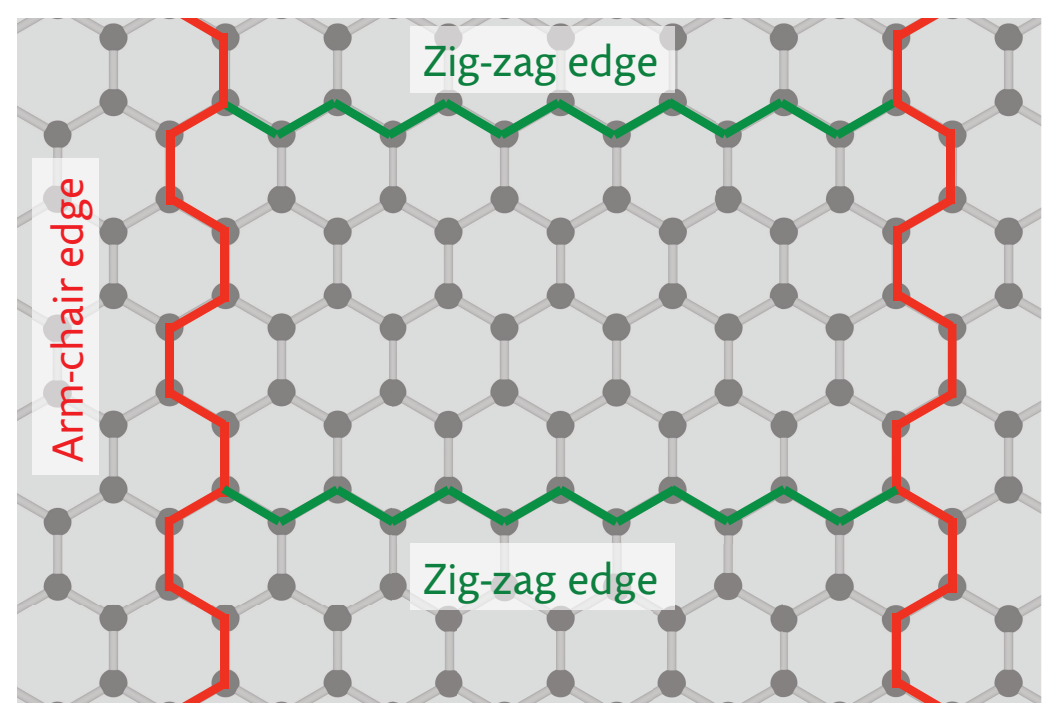

Figure 1.5: 2D honeycomb structure with zigzag edges (green lines) and armchair edges (red lines).

inversion symmetry, it can support an intrinsic polarization, which creates an internal electric field that can drive a transfer of electrons between the edges [41]. This form of "self-doping" in general promotes the metallic character of the edges. In chemistry, metallic TMD edges have been identified as sites of increased catalytic activity.

The nature of the edge states depends not only on the crystal structure, but also on the way it is terminated at the edge. As an example, Fig. 1.5 shows the two basic edge orientations of the honeycomb lattice: the zigzag edge and the armchair edge. For a monatomic 2D material such as graphene, the top and bottom zigzag edges are identical, as are the left and right armchair edges. In contrast, for a diatomic $2 \mathrm{D}$ material $A B$ such as $h$-BN or $\mathrm{MX}_{2}$, the top zigzag edge is terminated by $A$ atoms, whereas the bottom zigzag edge is terminated by $B$ atoms, which makes the two edges electronically different. The left and right armchair edges are still identical though.

In chapter 5 we develop a formalism suitable for calculating the electronic structure of a semi-infinite 2D layer, which is terminated by a single edge. This enables us to calculate the electronic properties of the states belonging to a single edge, without interference from other edges. The M-terminated and the X-terminated zigzag edges of $\mathrm{MX}_{2}$ show a markedly different structure of edge states with energies in the $\mathrm{MX}_{2}$ band gap. Whereas some of these states can be characterized as "dangling bond" states on the $\mathrm{M}$ or $\mathrm{X}$ atoms, others result from a more subtle change in the binding of atoms at or near the edge. Most of the edge orientations, including the zigzag orientations, result in metallic edges, with the exception of the armchair orientation, which gives semiconducting edges. 
As the edge states are not topologically protected, it makes sense to study how the states are affected by structural modifications of the edges. This is best done at the first-principles density-functional-theory (DFT) level, which is not yet possible with the formalism discussed in chapter 5, as so far that is implemented at the tight-binding level only. In our DFT calculations we are forced to use a nanoribbon geometry, where the ribbon is terminated by two edges. These calculations are described in chapter 6 . Using the guidance provided by the tight-binding calculations of chapter 5 one can disentangle the electronic structure of the two edges. Not surprisingly, the dangling-bond edge states are sensitive to structural and chemical changes at the edges, but the other edges states are remarkably robust.

Recent experimental techniques have made possible the materialization of nanoribbons of varying widths with almost smoothly defined edges [42, 43]. Control of growth conditions should enable control of the edge terminations. The boundaries between $2 \mathrm{D} \mathrm{MX}_{2}$ crystal grains typically also show an abundance of 1D metallic states. Often considered to be a nuisance in the growth of 2D semiconductors, such grain boundaries could provide a playground for 1D physics.

\subsection{Computational Methods}

In this thesis, we perform DFT calculations using the Vienna Ab-initio Simulation Package (VASP) [44, 45, 46, 47]. This program solves the Kohn-Sham equations numerically to find the total energy of a given system. To solve these equations efficiently the projector augmented wave method is used. In the following sections, a brief introduction to DFT is given, and to the density functionals used in this thesis.

\subsubsection{Density Functional Theory}

Density functional theory (DFT) is based upon the Hohenberg-Kohn theorems [48, 49], which state 1) that the ground-state total energy is a universal functional of the one-particle electronic density, and 2) that functional has a minimum for the exact ground state density. Kohn and Sham developed DFT into a practical computational scheme by mapping the real interacting electron system with electrons exposed to a real potential, onto a fictitious independent electron system with electrons subjected to an effective potential that is tuned such that the electron densities of the fictitious and the real systems are identical $[50,51,52,53]$. The many-particle wave function of the independent electron system is a Slater determinant of one-electron orbitals $\phi_{n}(\mathbf{r})$ that obey the eigenvalue equations $[53,54]$

$$
\left\{-\frac{\hbar^{2}}{2 m} \nabla^{2}+V_{\text {eff }}(\mathbf{r})\right\} \phi_{n}(\mathbf{r})=\epsilon_{n} \phi_{n}(\mathbf{r}),
$$


with the effective potential

$$
V_{\text {eff }}(\mathbf{r})=V_{\text {ext }}(\mathbf{r})+e^{2} \int \frac{n(\mathbf{r})}{\left|\mathbf{r}-\mathbf{r}^{\prime}\right|} d \mathbf{r}+\frac{\delta E_{x c}[n(\mathbf{r})]}{\delta n(\mathbf{r})} .
$$

Here $V_{\text {ext }}(\mathbf{r})$ is the "external" potential (usually the Coulomb potentials of the nuclei present in the system) and $E_{x c}[n]$ is a universal functional containing the effects of exchange and correlation interactions between the electrons. The density of the independent electron system is given by the usual expression

$$
n(\mathbf{r})=\sum_{n}^{N}\left|\phi_{n}(\mathbf{r})\right|^{2}
$$

Equations (1.3)-(1.5) constitute a set of non-linear equations that have to be solved iteratively. One usually starts by choosing a trial density $n_{0}(\mathbf{r})$ to calculate the potential $V_{\text {eff }}(\mathbf{r})$, Eq. (1.4), which is then used to calculate the orbitals $\phi_{n}(\mathbf{r})$, Eq. (1.3). These are then used to generate a new density via Eq. (1.5). The loop is repeated until the density is converged, i.e., it does not change anymore between cycles. Convergence is usually monitored by the total energy

$$
\begin{aligned}
E_{\mathrm{tot}} & =-\frac{\hbar^{2}}{2 m} \sum_{n=1}^{N}\left\langle\phi_{n}\left|\nabla^{2}\right| \phi_{n}\right\rangle+\frac{e^{2}}{2} \int \frac{n(\mathbf{r}) n\left(\mathbf{r}^{\prime}\right)}{\left|r-r^{\prime}\right|} d \mathbf{r} d \mathbf{r}^{\prime} \\
& +\int v_{e x t} n(\mathbf{r}) d \mathbf{r}+E_{x c}[n(\mathbf{r})] .
\end{aligned}
$$

This is the Kohn-Sham energy functional, which is variational. If each term in this functional would be known explicitly, we would be able to obtain the exact ground state density and total energy of any interacting many-electron system. Unfortunately, there is one unknown term, the exchange-correlation $(x c)$ functional $\left(E_{x c}\right)$. $E_{x c}$ includes the non-classical aspects of the electron-electron interaction along with the component of the kinetic energy of the real system different from the fictitious non-interacting system. It is necessary to approximate $E_{x c}$, which is the focus of the next section.

\subsubsection{Exchange and correlation functionals}

Since the birth of DFT, approximative exchange-correlation functionals have been both the strength and the Achilles heel of DFT calculations. By now there is a long list of functionals at various levels of complexity. In the following a short description is given of those functionals that are used in this thesis.

In the local density approximation (LDA) an inhomogeneous system is divided into infinitesimal volumes, and the electron density in each of the volumes is taken to be constant. The $x c$ energy for the density within each volume is then assumed to be the $x c$ energy obtained from the uniform electron gas of that density. Thus, the 
total $x c$ energy of the system can be written as [50]

$$
E_{x c}^{\mathrm{LDA}}[n]=\int n(\mathbf{r}) \varepsilon_{x c}(n(\mathbf{r})) d \mathbf{r},
$$

where $\varepsilon_{x c}$ is the $x c$ energy per particle of the interacting uniform electron gas of density $n(\mathbf{r})$. In practice, exchange and correlation are treated separately. The analytical expession for the exchange energy is known exactly $[55,56]$

$$
E_{x}^{\mathrm{LDA}}[n]=-\frac{3}{4}\left(\frac{3}{\pi}\right)^{1 / 3} \int n^{4 / 3}(\mathbf{r}) d \mathbf{r} .
$$

The correlation energy is given by a parametrized expression, with parameters obtained by fitting to numerical results on the correlation energy of the interacting uniform electron gas at different densities, derived from quantum Monte Carlo calculations $[55,56]$. Strictly speaking, the LDA is valid only for densities whose variations have a small amplitude or a long wave length. Neither of these is true in atoms, molecules, and solids, but experience has shown that the LDA works surprisingly well for these systems. In general, LDA tends to overbind somewhat, i.e., overestimate cohesive energies and underestimate lattice constants [50]. The overbinding problems of LDA become more severe for weakly bonded systems, such as van der Waals (vdW) or hydrogen bonded systems [57, 58].

As the next level of improvement upon the LDA, Eq. 1.7, it seems obvious to include terms based upon the gradient of the density, $\nabla n(\mathbf{r})$. In the generalized gradient approximation (GGA) this is written as

$$
E_{x c}^{\mathrm{GGA}}[n]=\int n(\mathbf{r}) \varepsilon_{x c}(n(\mathbf{r})) F_{x c}^{\mathrm{GGA}}(n(\mathbf{r}), \nabla n(\mathbf{r})) d \mathbf{r},
$$

where $F_{x c}^{\mathrm{GGA}}$ describes the enhancement or suppression of the LDA value due to a local variation of the density. Becase of their dependence on $\nabla n(\mathbf{r})$, GGAs are called "semi-local" functionals.

A straight-forward expansion of $E_{x c}$ in derivatives of the density can lead to expressions that do not converge monotonically, contain divergencies, or disobey important sum rules (the $x c$-hole density should integrate to -1 , for instance). To avoid these problems one uses expressions that (implicitly) sum over an infinite series of similar terms, and force them to obey physical constraints, such as sum rules and important low/high density limits. There is no unique way of doing this, and consequently a number of different GGA functionals have been developed. One of the most popular GGAs is the Perdew-Burke-Ernzerhof (PBE) functional [59]. Its exchange part has the form

$$
F_{x}^{\mathrm{PBE}}(s)=1+\kappa-\frac{\kappa}{\left(1+\mu s^{2} / \kappa\right)} .
$$


Here $s$ is a dimensionless reduced gradient.

$$
s=\frac{|\nabla n(\mathbf{r})|}{2\left(3 \pi^{2}\right)^{1 / 3} n(\mathbf{r})^{4 / 3}} .
$$

The values for $\kappa=0.804$ and $\mu=0.21951$ in the PBE expression are obtained from physical constraints, which makes the expression non-empirical. If the density gradient is zero, $s=0$ and $F_{x}^{G G A}(0)=1$, we return to the LDA exchange.

The PBE functional gives markedly better binding and cohesive energies than the LDA for strongly bonded systems, which is at the basis of its popularity in chemistry [59]. For many systems it also improves upon the description of the equilibrium geometry (bond lengths and angles). Sometimes it underbinds somewhat; for instance, the in-plane equilibrium lattice constant of $\mathrm{MoS}_{2}$ is $1-2 \%$ too large as compared to experiment. The underbinding becomes severe for weakly bonded systems, where $\mathrm{vdW}$ interactions play an important role. For instance, PBE essentially fails to give any bonding between the graphene layers in graphite [60], or between a $\mathrm{MoS}_{2}$ layer and the $\mathrm{Au}(111)$ surface (chapter 2).

Density functionals based on the local density approximation (LDA) [56] or the semilocal generalized gradient approximation (GGA) [55, 59] do not account for long-range correlations, which are resposible for vdW interactions. The 2D layers of the materials discussed in Sec. 1.1 interact through vdW interactions, and GGA/PBE completely fails to capture the attraction between such layers. In contrast, LDA yields reasonably good results for equillibrium distances and binding energies between graphene layers, between $h$-BN layers, and between $h$-BN or graphene and metal(111) surfaces. But this is accidental and due to the fact that LDA overestimates the range of the exchange interactions, and not because LDA correctly incorporates vdW interactions [61, 62].

Van der Waals density functionals (vdWDF) have been developed that explicitly model non-local vdW correlations [63, 64, 65]. The $x c$ functional is divided into three parts

$$
E_{x c}=E_{c}^{\mathrm{loc}}+E_{c}^{\mathrm{vdW}}+E_{x},
$$

where $E_{c}^{\text {loc }}$ is a local correlation energy, $E_{c}^{\mathrm{vdW}}$ describes nonlocal electron-electron correlations, and $E_{x}$ is the exchange energy. The LDA expression is used for $E_{c}^{\text {loc }}$, and for $E_{c}^{\mathrm{vdW}}$ the vdW kernel developed by Dion et al. [63]

$$
E_{c}^{\mathrm{vdW}}=\frac{1}{2} \int n(\mathbf{r}) \phi\left(\mathbf{r}, \mathbf{r}^{\prime}\right) n\left(\mathbf{r}^{\prime}\right) d \mathbf{r} d \mathbf{r}^{\prime}
$$

In this thesis the exchange parts of the optB88 and optB86b GGA functionals are used for $E_{x}$. The B88 exchange enhancement factor is

$$
F_{x}^{\mathrm{B} 88}(s)=1+\mu s^{2} /(1+\beta s \operatorname{arcsinh}(c s)),
$$

with $c=2^{4 / 3}\left(3 \pi^{2}\right)^{1 / 3}, \mu=0.2743$, and $\beta=9 \mu(6 / \pi)^{1 / 3} / 2 c$. For optB86b, the exchange 
(a)

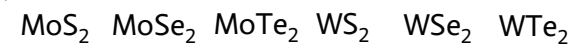

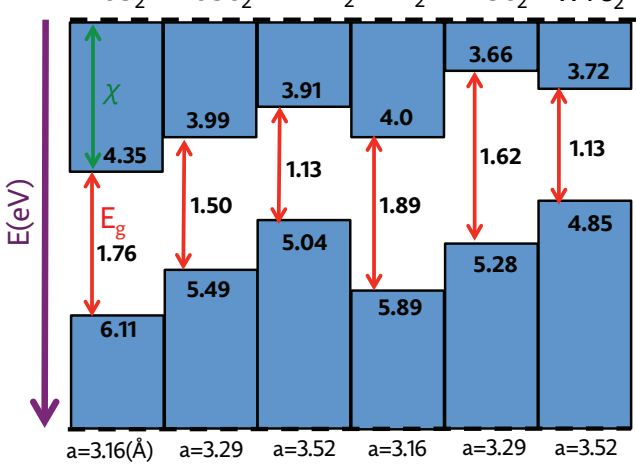

(b)
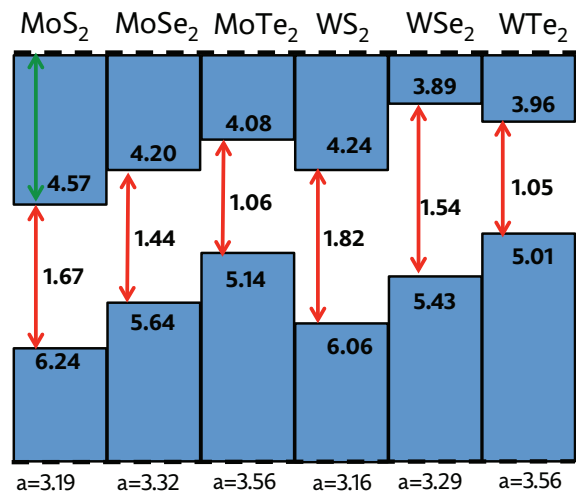

Figure 1.6: Ionization potentials, electron affinities, band gaps, and in-plane lattice constants of $\mathrm{MX}_{2}$ monolayers calculated with (a) the optB86b-vdWDF and (b) the optB88-vdWDF functionals.

factor is

$$
F_{x}^{\mathrm{B} 86 \mathrm{~b}}=1+\frac{\mu s^{2}}{\left(1+\mu s^{2}\right)^{4 / 5}}
$$

with $\mu=0.1234[63,64,66,67]$. These expressions give similar results as the exchange part of the PBE functional, Eq. 1.10, but used in the vdWDF, both optB88 and optB86b yield better equilibrium structures for graphite [60]. In general, vdWDFs give sensible values for the equilibrium structure of layered materials, where the interlayer bonding is $\mathrm{vdW}$, as well as for the interlayer binding energy. The same holds for 2D layers adsorbed on metal substrates, see chapter 2. For strongly bonded systems the vdWDF is generally not an improvement over PBE.

Figure 1.6 shows the optimized in-plane lattice constants, the ionization potentials, the electron affinities and the band gaps of TMD 2D layers, calculated with the optB86b-vdWDF and the optB88-vdWDF functionals. The optB86b-vdWDF in-plane lattice constant is generally $\sim 1 \%$ smaller than the optB88-vdWDF lattice constant, and is in good agreement with the experimental data, see Table 4.2 in chapter 4 . The optB86b-vdWDF band gap is 0.06-0.09 eV larger than the optB88-vdWDF band gap (very likely because of the smaller lattice constant), and is in reasonable agreement with the experimental optical band gap, see Table 4.2 in chapter 4 . The difference between the two functionals in ionization potentials is $\sim 0.15 \mathrm{eV}$ and in electron affinities $\sim 0.2 \mathrm{eV}$. Fortunately this does not result in a corresponding uncertainty in the $\mathrm{SBH}$, Eq. 1.2, as the metal work functions $W_{M}$ show a similar dependence, and the interface potential step $\Delta V$ is almost functional independent. 


\section{2}

\section{A first-principles study of van der Waals interactions and lattice mismatch at $\mathrm{MoS}_{2} /$ metal interfaces}

* We explore the adsorption of $\mathrm{MoS}_{2}$ on a range of metal substrates by means of firstprinciples density functional theory calculations. Including van der Waals forces in the density functional is essential to capture the interaction between $\mathrm{MoS}_{2}$ and a metal surface, and obtain reliable interface potential steps and Schottky barriers. Special care is taken to construct interface structures that have a mismatch between the $\mathrm{MoS}_{2}$ and the metal lattices of $<1 \% . M o S_{2}$ is chemisorbed on the early transition metal Ti, which leads to a strong perturbation of its (electronic) structure and a pinning of the Fermi level $0.54 \mathrm{eV}$ below the $\mathrm{MoS}_{2}$ conduction band due to interface states. MoS 2 is physisorbed on Au, where the bonding hardly perturbs the electronic structure. The bonding of $\mathrm{MoS}_{2}$ on other metals lies between these two extreme cases, with interface interactions for the late $3 \mathrm{~d}$ transition metals $\mathrm{Co}, \mathrm{Ni}$, $\mathrm{Cu}$ and the simple metal $\mathrm{Mg}$ that are somewhat stronger than for the late $4 \mathrm{~d} / 5 \mathrm{~d}$ transition metals Pd, Ag, Pt and the simple metal Al. Even a weak interaction, such as in the case of $A l$, gives interface states, however, with energies inside the $M_{0} S_{2}$ band gap, which pin the Fermi level below the conduction band.

\subsection{Introduction}

Transition metal dichalcogenides (TMDs) such as molybdenum disulfide $\left(\mathrm{MoS}_{2}\right)$ have layered structures, where the atoms within a TMD monolayer form a covalently bonded planar network, and the interaction between these layers is a weak, van der

${ }^{*}$ This chapter has been published as: M. Farmanbar and G. Brocks, A first-principles study of van der Waals interactions and lattice mismatch at $\mathrm{MoS}_{2}$ / metal interfaces, Phys. Rev. B 93, 085304 (2016). 
Waals interaction [4, 21]. A monolayer of $\mathrm{MoS}_{2}$ consists of a layer of molybdenum atoms sandwiched between two layers of sulfur atoms. $\mathrm{MoS}_{2}$ monolayers can be exfoliated through micro-mechanical cleavage, similar to graphene or boron nitride [68]. Unlike graphene (a metal), or boron nitride (an insulator), $\mathrm{MoS}_{2}$ is a semiconductor. Moreover, whereas bulk $\mathrm{MoS}_{2}$ has an indirect band gap $(1.2 \mathrm{eV})$, monolayer $\mathrm{MoS}_{2}$ has a direct band gap ( 1.8-1.9 eV), and shows a strong optical absorption and luminescence $[25,69]$. At present $\mathrm{MoS}_{2}$, and TMDs in general, are vehemently pursued as promising materials for applications in electronics and optoelectronics $[69,70]$.

Contacting $\mathrm{MoS}_{2}$ to metal electrodes proves to be a problem; it tends to produce unexpectedly high interface resistances, indicative of a high Schottky barrier at the interface $[71,72,73,74,75,76]$. A high barrier could be caused by strong interface bonding creating interface states that pin the Fermi level [77], or by weak bonding creating a potential step due to Pauli repulsion at the interface [40, 78]. The nature of the interaction at the $\mathrm{MoS}_{2} /$ metal interface is far from trivial. On the one hand, one could argue that, as $\mathrm{MoS}_{2}$ has no dangling bonds at its surface, its interaction with metal substrates should be weak and van-der-Waals-like. On the other hand, many metal species form (di)chalcogenide compounds [25, 69, 79, 80], and when adsorbing $\mathrm{MoS}_{2}$ onto a metal substrate, there could be a competition between the metal surface and the Mo atoms for interacting with the sulfur atoms at the interface. In that case, not only the $\mathrm{MoS}_{2}$ / metal bonding would be a much stronger chemical bonding, but also the structure and electronic structure of the $\mathrm{MoS}_{2}$ adsorbate could be significantly perturbed.

In this paper we explore the adsorption of $\mathrm{MoS}_{2}$ on a variety of metal substrates by means of first-principles density functional theory (DFT) calculations, following up on work briefly reported in a short paper [77]. Previous DFT studies have concentrated foremost on the Schottky barrier formed at $\mathrm{MoS}_{2} /$ metal interfaces using the local density approximation (LDA) [55, 81, 82, 83, 84, 85]. LDA gives a reasonable description of the adsorption of graphene and $h$-BN on metal surfaces, but such results cannot be generalized to other systems, as it is known that LDA often leads to an unrealistic overbinding $[86,87,88,89,90,91,92,78,40]$. Other studies have used a generalized gradient approximation (GGA) functional, such as PBE [59], which apparently works well for TMDs adsorbed on metals [93, 94, 77], although it generally gives bad results for weakly bonded systems [95, 60].

Here we focus on the interface interaction and its implications for the structure and electronic structure of the $\mathrm{MoS}_{2}$ adsorbate and the Schottky barrier. We choose a wide range of metal substrates: the (111) surfaces of $\mathrm{Al}, \mathrm{Ni}, \mathrm{Cu}, \mathrm{Pd}, \mathrm{Ag}, \mathrm{Pt}$ and $\mathrm{Au}$, and the (0001) surfaces of $\mathrm{Mg}, \mathrm{Ti}$, and $\mathrm{Co}$, which are expected to have a wide range of interaction strengths with the adsorbate. As the interface interaction can vary from weak (physisorption) to strong (chemisorption), it is a priori not clear which DFT functional describes such bonding. We test and compare results obtained with a van der Waals functional, designed to describe weak, van der Waals, interactions $[63,65,64]$, to results obtained with GGA and LDA functionals, which are conven- 
tionally used to describe chemical bonding. We assess the importance of van der Waals interactions for the interface interaction, and evaluate its effect on the structure and electronic structure of the $\mathrm{MoS}_{2}$ adsorbant.

We consider the situation where a $\mathrm{MoS}_{2}$ layer is adsorbed as a whole on a metal substrate, making it more likely that the integrity of the $\mathrm{MoS}_{2}$ layer is preserved in the adsorption process. If the $\mathrm{MoS}_{2} /$ metal interaction is not too strong, and the $\mathrm{MoS}_{2}$ and metal surface lattices are not matched, the interface structure is likely to be incommensurable. In a supercell calculation one is forced to approximate such a structure by a commensurable one. Previous calculations have used small supercells, where in some cases appreciable artificial strain is generated because of the mismatch between the $\mathrm{MoS}_{2}$ and the metal surface lattices [81, 83, 84, 85]. We apply a strategy for choosing supercells such that the artificial strain is minimal, and test the influence of strain on the electronic properties of the interface.

This paper is organized as follows. Section 2.2 describes the DFT calculations, comparing different functionals in Sec. 2.2.2 and discussing the effect of lattice mismatch in Sec. 2.2.3. Results are discussed in Sec. 6.3, with the metal/ $\mathrm{MoS}_{2}$ interaction in Sec. 2.3.1 and its effects on the interface potential step and the Schottky barrier in Sec. 2.3.2. Strong chemisorption is discussed in more detail in Sec. 2.3.3, and a summary and the conclusions are presented in Sec. 2.4.

\subsection{Calculations}

\subsubsection{Computational Methods}

We calculate ground-state energies and optimize geometries at the density functional theory (DFT) level, using projector-augmented waves (PAWs) as implemented in the VASP code [44, 45, 46, 47]. The plane-wave kinetic-energy cutoff is set at $400 \mathrm{eV}$. The surface Brillouin zone is integrated with the Methfessel-Paxton technique using a smearing parameter of $0.05 \mathrm{eV}$ [96], and a $k$-point sampling grid with a spacing of $0.01 \AA^{-1}$. The $\mathrm{MoS}_{2} /$ metal interface is modeled as a slab of 4-6 layers of metal atoms with one or two layers of $\mathrm{MoS}_{2}$ adsorbed on one side and a vacuum region of $\sim 12 \AA$. The in-plane supercell is chosen such as to minimize the mismatch between the $\mathrm{MoS}_{2}$ and metal lattices, which is discussed in more detail in Sec. 2.2.3. A dipole correction is applied to avoid spurious interactions between periodic images of the slab [97]. We allow the positions of the atoms to relax until the force on each atom is smaller than $0.01 \mathrm{eV}^{-1}$, except for the bottom layer of metal atoms, whose positions are kept fixed. The electronic self-consistency criterion is set to $10^{-5} \mathrm{eV}$.

It is well known that commonly used DFT exchange-correlation functionals, based upon LDA [55] or GGA [59], give decent descriptions of covalent and ionic bonding, but they may fail for weakly bonded systems, as such functionals do not contain a description of van der Waals interactions. For example, GGA functionals such as PW91 or PBE [59], do not capture the bonding between $h$-BN or graphene layers, nor that between $h$-BN or graphene and transition metal(111) surfaces $[60,98]$. A 


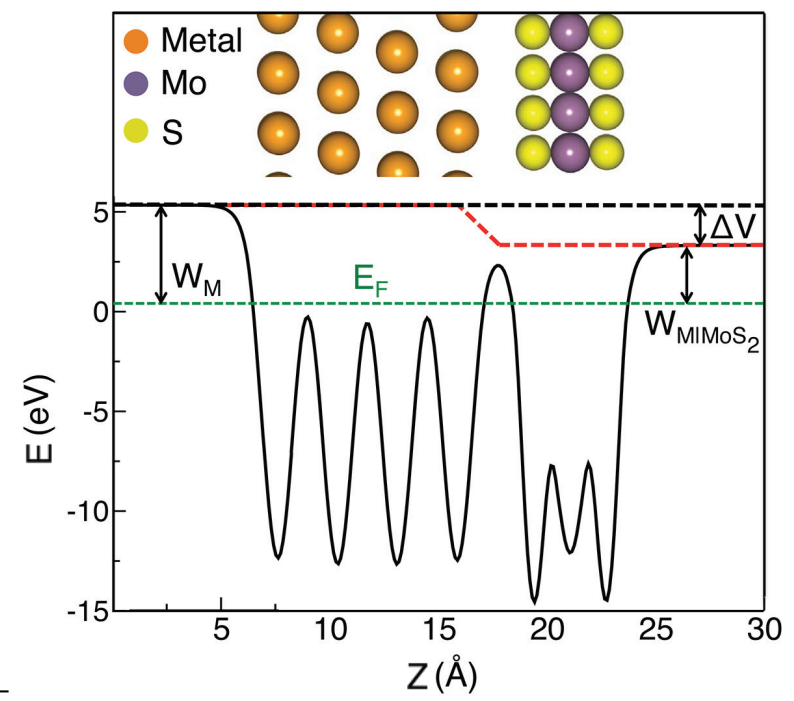

Figure 2.1: (Color online) Side view of metal/ $\mathrm{MoS}_{2}$ structure with corresponding plane-averaged electrostatic potential $\bar{V}(z)$ and $E_{F}$ the Fermi level. The interface potential step $\Delta V$ is reflected in the difference between the work function on the metal side $W_{\mathrm{M}}$ and on the $\mathrm{MoS}_{2}$ side $W_{\mathrm{M} \mid \mathrm{MoS}_{2}}$.

priori we don't know how important van der Waals interactions are in the bonding between $\mathrm{MoS}_{2}$ and a metal surface. In Sec. 2.2.2 we compare results obtained using a van der Waals density functional (vdW-DF) [63, 64, 65], with results obtained with GGA and LDA functionals.

One way of visualizing bonding at a $\mathrm{MoS}_{2}$ / metal interface is by the electron density difference

$$
\Delta n(\mathbf{r})=n_{\mathrm{M} \mid \mathrm{MoS}_{2}}(\mathbf{r})-n_{\mathrm{M}}(\mathbf{r})-n_{\mathrm{MoS}_{2}}(\mathbf{r}),
$$

where $n_{\mathrm{M} \mid \mathrm{MoS}_{2}}(\mathbf{r}), n_{\mathrm{M}}(\mathbf{r})$, and $n_{\mathrm{MoS}_{2}}(\mathbf{r})$ are the electron densities of $\mathrm{MoS}_{2}$ adsorbed on the metal, of the metal surface and of the free standing $\mathrm{MoS}_{2}$, respectively. The system as a whole is neutral, and $\Delta n(\mathbf{r})$ is localized around the metal/MoS $\mathrm{M}_{2}$ interface, i.e. $\Delta n(\mathbf{r}) \rightarrow 0$ for $\mathbf{r}$ sufficiently far from the interface. Solving the Poisson equation with $\Delta n(\mathbf{r})$ as source then gives a potential step across the interface

$$
\Delta V=\frac{e^{2}}{\epsilon_{0} A} \iiint z \Delta n(\mathbf{r}) d x d y d z .
$$

Here $z$ is the direction normal to the interface, $A$ is the interface area, and $\Delta V$ is the difference between the asymptotic values of the potential left and right of the interface. 
(a)

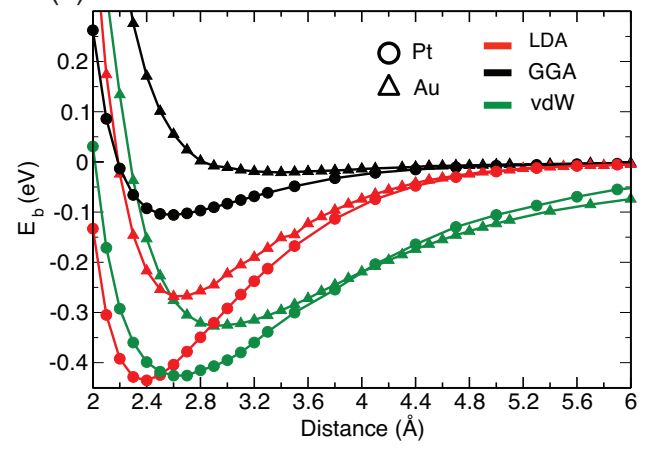

(b)

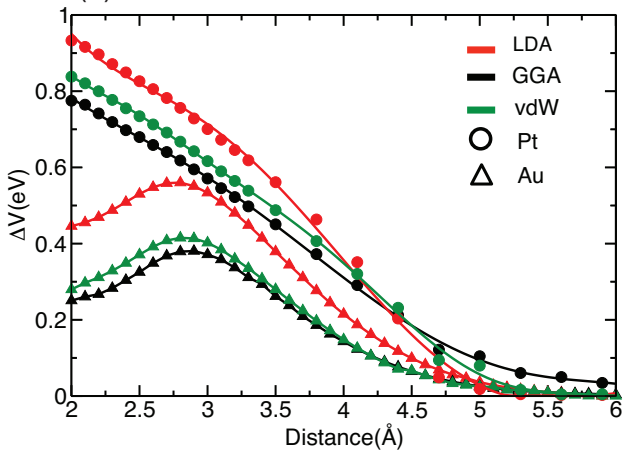

Figure 2.2: (Color online) (a) Binding energy curves $E_{b}(d)$ of $\mathrm{MoS}_{2}$ on $\mathrm{Au}$ and $\mathrm{Pt}(111)$, calculated with the GGA/PBE (black), opt88b-vdw-DF (green), and LDA (red) functionals. (b) Interface potential steps $\Delta V(d)$ for $\mathrm{MoS}_{2}$ on $\mathrm{Au}$ and $\mathrm{Pt}(111)$, calculated with the three functionals. $d$ is the distance between the top metal and the bottom sulfur layers.

Figure 2.1 also illustrates an alternative expression for the potential step

$$
\Delta V=W_{\mathrm{M}}-W_{\mathrm{M} \mid \mathrm{MoS}_{2}}
$$

where $W_{\mathrm{M}}, W_{\mathrm{M} \mid \mathrm{MoS}_{2}}$ are the work functions of the clean metal surface, and of the metal surface covered by $\mathrm{MoS}_{2}$, respectively. A practical way of obtaining work functions from DFT calculations is to track the plane-averaged electrostatic (Hartree) potential $\bar{V}(z)$ into the vacuum, see Fig. 2.1, where typically the asymptotic value is reached with a few $\AA$ from the surface. In converged calculations the expressions of Eqs. 2.2 and 2.3 give results that are with a few meV of one another.

The Schottky barrier height for electrons is defined as

$$
\Phi_{\mathrm{n}}=E_{F}-\chi_{\mathrm{MoS}_{2}}
$$

with $E_{F}$ the Fermi level and $\chi_{\mathrm{MoS}_{2}}$ the electron affinity of $\mathrm{MoS}_{2}$, both defined as distances to the vacuum level, i.e., positive numbers. There are several ways to extract the Schottky barrier height from $\mathrm{MoS}_{2} /$ metal slab calculations.

One could determine $\Phi_{\mathrm{n}}$ by measuring $E_{F}-\chi_{\mathrm{MoS}_{2}}$ in the band structure or in the density of states of the $\mathrm{MoS}_{2} /$ metal slab, as in Refs. [82, 83, 84] and [85]. In order to identify the $\mathrm{MoS}_{2}$ related states, one needs to calculate the amplitudes of the projections of the wave functions of the slab on the $\mathrm{MoS}_{2}$ layer. There is always some arbitrariness involved in such a projection if the adsorbate and the substrate are in close connection. In addition, identification of states belonging to the adsorbate is possible only if its electronic structure is not significantly perturbed in the adsorption process, which is only the case if the adsorbate is (weakly) physisorbed on the 
substrate [40]. In practice we find that this procedure for obtaining the Schottky barrier height at $\mathrm{MoS}_{2} /$ metal interfaces is not sufficiently accurate when applied to the projected density of states, and of practical use only when applied to the projected band structure of a small supercell.

An alternative way of locating the conduction band edge $\chi_{\mathrm{MoS}_{2}}$ in a $\mathrm{MoS}_{2} /$ metal slab calculation, without having to resort to wave function projections, is by aligning the core levels of the Mo or $\mathrm{S}$ atoms in the slab with the corresponding core levels in free-standing $\mathrm{MoS}_{2}$. It allows us to compare the densities of states of free-standing and adsorbed $\mathrm{MoS}_{2}$, see Sec. 2.3.2, and, in principle, this procedure also allows for calculating the Schottky barrier height. Of course, this only makes sense if the $\mathrm{MoS}_{2}$ electronic structure is not perturbed too strongly by the adsorption.

Alternatively, the two quantities $E_{F}$ and $\chi_{\mathrm{MoS}_{2}}$ on the right-hand side of Eq. 2.4 are are easily obtained in separate calculations on an $\mathrm{MoS}_{2} /$ metal slab and a freestanding $\mathrm{MoS}_{2}$ layer, respectively. On the $\mathrm{MoS}_{2}$ side of the slab we have $E_{F}=$ $W_{\mathrm{M} \mid \mathrm{MoS}_{2}}$, see Fig. 2.1. Convergence as a function of slab thickness is usually faster if we use Eq. 2.3, and extract $\Delta V$ from a calculation on an $\mathrm{MoS}_{2} /$ metal slab, and $W_{\mathrm{M}}$ from a separate calculation on a clean metal slab. The Schottky barrier height is then calculated as

$$
\Phi_{\mathrm{n}}=W_{\mathrm{M}}-\chi_{\mathrm{MoS}_{2}}-\Delta V .
$$

Of course, if the $\mathrm{MoS}_{2}$ electronic structure is very strongly perturbed by adsorption, one has to reconsider the definition of the Schottky barrier, see Sec. 2.3.3. In the following the potential step $\Delta V$ is used to characterize the $\mathrm{MoS}_{2} /$ metal interface, along with the binding energy and the structure.

\subsubsection{Comparison of DFT Functionals}

Materials such as graphite, $h$ - $\mathrm{BN}$ and $\mathrm{MoS}_{2}$ have a layered structure, where the atoms within one layer form strong covalent bonds, but the interaction between the layers consists of weak, van der Waals, forces. Common GGA functionals, such as PBE [59], lack a description of van der Waals interactions, which results in a severe underestimation of the interlayer binding energy in graphite and $h-\mathrm{BN}$, and an overestimation of the interlayer bonding distance $[60,95]$. Similar problems are encountered when graphene or $h$-BN are adsorbed on a metal substrate [87, 89, 90, 91, 92]. The LDA functional also lacks a description of van der Waals interactions, but it, somewhat fortuitously, gives reasonable binding energies and geometries for graphite, $h$-BN, and for the adsorption of these materials on metals [40, 86, 88, 78]. In general however, the LDA functional tends to overestimate binding energies, which is regularly accompanied by an underestimation of the bonding distance.

Many of these problems are mitigated when using vdW-DFs [99, 100], which, for instance, describe the bonding in graphite very well [60]. The exchange-correlation energy in a vdW-DF takes the form

$$
E_{\mathrm{xc}}=E_{\mathrm{x}}+E_{\mathrm{c}}^{\mathrm{vdW}}+E_{\mathrm{c}}^{\mathrm{loc}}
$$


Table 2.1: Equilibrium bonding distance $d_{e q}$, binding energy $E_{b}$, and interface potential step $\Delta V$, for $\mathrm{MoS}_{2}$ on metal (111) surfaces, calculated with different functionals.

\begin{tabular}{|c|c|c|c|c|c|c|c|c|c|c|c|c|}
\hline & \multicolumn{3}{|c|}{$\mathrm{Au}$} & \multicolumn{3}{|c|}{$\mathrm{Ag}$} & \multicolumn{3}{|c|}{$\mathrm{Pd}$} & \multicolumn{3}{|c|}{$\mathrm{Pt}$} \\
\hline & $d_{e q}(\AA)$ & $\Delta V(\mathrm{eV})$ & $E_{b}(\mathrm{eV})$ & $d_{e q}(\AA)$ & $\Delta V(\mathrm{eV})$ & $E_{b}(\mathrm{eV})$ & $d_{e q}(\AA)$ & $\Delta V(\mathrm{eV})$ & $E_{b}(\mathrm{eV})$ & $d_{e q}(\AA)$ & $\Delta V(\mathrm{eV})$ & $E_{b}(\mathrm{eV})$ \\
\hline LDA & 2.6 & 0.54 & -0.27 & 2.5 & 0.10 & -0.33 & 2.2 & 0.50 & -0.69 & 2.4 & 0.85 & -0.43 \\
\hline PBE & 3.3 & 0.38 & -0.02 & 2.8 & 0.10 & -0.08 & 2.3 & 0.34 & -0.25 & 2.6 & 0.66 & -0.11 \\
\hline $\mathrm{vdW}$-DF & 2.9 & 0.41 & -0.33 & 2.8 & 0.11 & -0.35 & 2.4 & 0.30 & -0.54 & 2.6 & 0.71 & -0.43 \\
\hline
\end{tabular}

where $E_{\mathrm{x}}, E_{\mathrm{c}}^{\mathrm{loc}}$ and $E_{\mathrm{c}}^{\mathrm{vdW}}$ describe the exchange part, and the local and nonlocal electron-electron correlations, respectively. For $E_{\mathrm{c}}^{\mathrm{vdW}}$ we use the vdW kernel developed by Dion et al. [63] and for $E_{\mathrm{c}}^{\text {loc }}$ the correlation part of the LDA functional. For the exchange part $E_{\mathrm{x}}$ we use the optB88 functional [64]. The opt88-vdW-DF has given good results for binding energies and geometries of graphite, $h$-BN, and the adsorption of these materials on metals $[60,78]$.

In the following we test the GGA/PBE, LDA, and opt88-vdW-DF functionals for the adsorption of $\mathrm{MoS}_{2}$ on metals. As test cases we use the $4 \mathrm{~d}$ and $5 \mathrm{~d}$ metals $\mathrm{Ag}, \mathrm{Au}$, $\mathrm{Pd}$, and Pt. We place a $\mathrm{MoS}_{2}$ monolayer on top of the (111) surface of these metals, choosing a $\sqrt{3} \times \sqrt{3} R 30^{\circ}$ in-plane $\mathrm{MoS}_{2}$ unit cell on top of a $2 \times 2$ (111) surface cell. The in-plane $\mathrm{MoS}_{2}$ lattice parameters are kept at their optimized values for a freestanding layer, and the in-plane metal lattice parameter is adapted accordingly. The size of the adaption is maximal for $\mathrm{Au}$, where it results in a compression of the inplane Au lattice by $4.2 \%$. The effects of this artificial strain are discussed in the next section.

Figure 2.2(a) shows the binding curves of $\mathrm{MoS}_{2}$ on $\mathrm{Au}$ and $\mathrm{Pt}(111)$ for the three functionals. The binding energy is defined as the total energy per $\mathrm{MoS}_{2}$ formula unit of the metal/ $\mathrm{MoS}_{2}$ slab minus the total energies of the clean metal slab and the free-standing $\mathrm{MoS}_{2}$ layer, as a function of the distance $d$ between the top layer of metal atoms and the bottom layer of sulfur atoms. For $\mathrm{MoS}_{2}$ on $\mathrm{Au}(111), \mathrm{PBE}$ gives virtually no bonding, and opt88-vdW-DF gives a sizable binding energy. The opt88vdW-DF result suggests that $\mathrm{MoS}_{2}$ is physisorbed on $\mathrm{Au}(111)$, with van der Waals interactions playing the decisive role in the bonding. PBE does not capture this at all. LDA gives a equilibrium binding distance that is $0.3 \AA$ smaller, and an equilibrium binding energy that is $32 \%$ larger.

For $\mathrm{MoS}_{2}$ on $\mathrm{Pt}(111)$ all three functionals give equilibrium bonding distances that are shorter than for $\mathrm{MoS}_{2}$ on $\mathrm{Au}(111)$, and a bonding that is stronger, which suggests that $\mathrm{MoS}_{2}$ may be weakly chemisorbed on $\mathrm{Pt}(111)$. PBE and opt88-vdW-DF give a similar equilibrium distance, although PBE captures only $26 \%$ of the binding energy, indicating that van der Waals interactions still play an important role here. LDA gives a similar binding energy as opt88-vdW-DF, but an equilibrium binding distance that is $0.2 \AA$ smaller.

Table 2.1 shows the equilibrium binding distances and energies obtained with the three functionals for $\mathrm{MoS}_{2}$ on $\mathrm{Au}, \mathrm{Ag}$, $\mathrm{Pd}$, and $\mathrm{Pt}(111)$. Treating the results for opt88vdW-DF as a benchmark, PBE is seen to severely underestimate binding energies, 
whereas LDA gives quite reasonable binding energies. LDA however gives binding distances that are up to $0.3 \AA$ shorter than those obtained with opt88-vdW-DF, in particular for cases where the bonding is weak, such as Au and Ag. In contrast, PBE gives binding distances that are similar to those obtained with opt88-vdW-DF, except for $\mathrm{Au}$, where PBE essentially fails to give any significant bonding.

Potential steps $\Delta V$ as a function of the distance $d$ between the top layer of metal atoms and the bottom layer of sulfur atoms, calculated according to Eq. 2.3, are shown in Fig. 2.2(b) for $\mathrm{Au}$ and Pt. The curves for the PBE and the opt88-vdWDF functionals are within $0.05 \mathrm{eV}$ of one another in the range $d=2.5-3 \AA$, whereas LDA gives a potential step that is $0.10-0.15 \mathrm{eV}$ higher. In view of the considerable differences in the binding curves for these three functionals, the differences in the potential steps are remarkably small. This is true for all metal substrates listed in Table 2.1.

In Ref. [40] the main contribution to the potential step in the adsorption of $h$-BN on metal substrates was attributed to Pauli repulsion. This can be modeled by an electron density that is obtained by anti-symmetrizing the product of the metal and the adsorbate wave functions. As long as these wave functions do not strongly depend on the functional, the electron density and the potential step are also relatively insensitive to the functional used. This is unlike the total energy, which for a given electron density is very dependent on the functional. For the potential step to be accurate it is however important to obtain the proper equilibrium binding distance $[86,88,78,40]$.

\subsubsection{Lattice Mismatch}

The absolute values of the binding energies given in Table 2.1 are much smaller than what one expects to find for true chemical bonding. The differences between the values obtained with PBE and opt88-vdW-DF indicate that van der Waals interactions play a significant role in the bonding. With such a weak metal/adsorbate bonding it is unlikely that the metal substrate can enforce its lattice periodicity onto the $\mathrm{MoS}_{2}$ overlayer. Therefore, a metal/ $\mathrm{MoS}_{2}$ interface very likely becomes incommensurable if the metal $/ \mathrm{MoS}_{2}$ lattice mismatch is substantial. In electronic structure calculations one is forced to use commensurable structures to model incommensurable systems. Obviously care must be taken to ensure that the artificial strain introduced this way, does not alter the electronic structure in an unrealistic way.

Based upon previous experience, we expect that modifying the in-plane lattice constant of a close-packed metal surface by a few percent only affects its electronic properties mildly [40,78, 86, 88]. In contrast, changing the lattice parameter of $\mathrm{MoS}_{2}$ by just one percent already alters the band gap by $\sim 0.1 \mathrm{eV}$, and changes it from direct to indirect. A larger change in the lattice parameter has an even more dramatic effect. Applying a tensile strain of $\sim 5 \%$ to $\mathrm{MoS}_{2}$ reduces the band gap by $\sim 1 \mathrm{eV}$ [101, 102, 103, 104, 105, 106].

As an example, the PBE optimized in-plane lattice parameters of $\mathrm{MoS}_{2}$ and $\mathrm{Au}(111)$ 


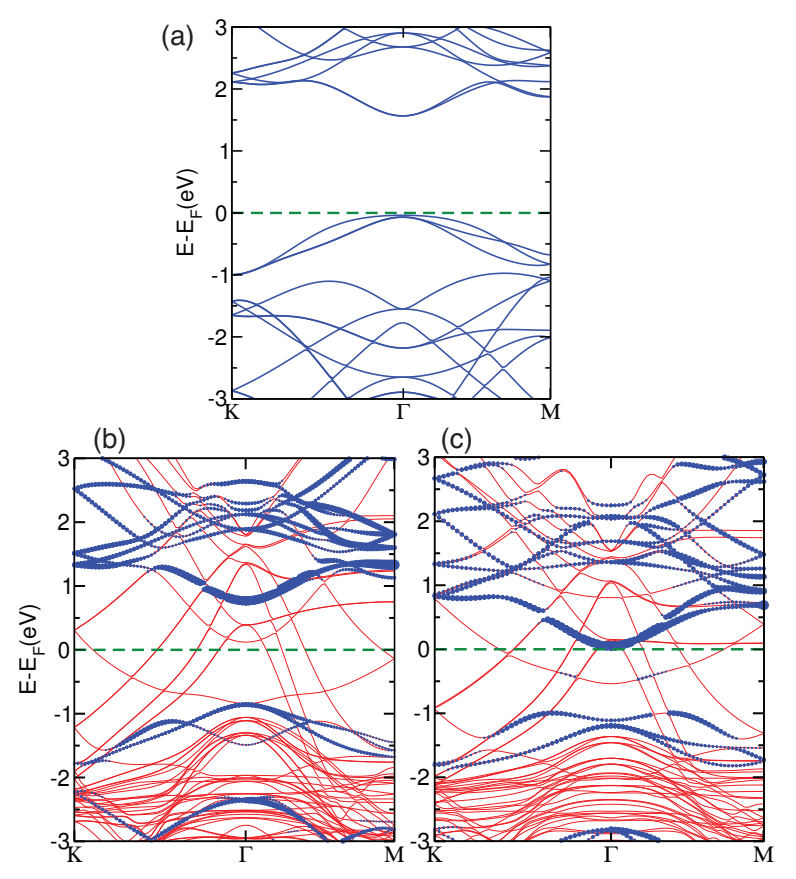

Figure 2.3: (Color online) (a) Band structure of a free-standing $\mathrm{MoS}_{2}$ monolayer in a $\sqrt{3} \times \sqrt{3}$ cell, where the direct band gap appears at $\Gamma$; (b) Band structure of $\mathrm{MoS}_{2} / \mathrm{Au}(111)$ with the in-plane Au lattice compressed by $4.2 \%$ to match the $\mathrm{MoS}_{2}$ lattice; the blue color indicates the weight of a projection of the wave functions on the $\mathrm{MoS}_{2}$ sites; (c) as (b) but with the $\mathrm{MoS}_{2}$ lattice stretched by $4.2 \%$ to match the $\mathrm{Au}(111)$ lattice.

are $3.19 \AA$ and $2.88 \AA$. Placing a $(\sqrt{3} \times \sqrt{3}) R 30^{\circ} \mathrm{MoS}_{2}$ cell on top of $2 \times 2 \mathrm{Au}(111)$ surface cell then leads to a lattice mismatch of $4.2 \%$. Figure $2.3(\mathrm{~b})$ shows the electronic band structure of $\mathrm{MoS}_{2} / \mathrm{Au}(111)$ where the in-plane $\mathrm{Au}(111)$ is compressed by $4.2 \%$ to match the lattice parameter of $\mathrm{MoS}_{2}$. As the interaction between $\mathrm{MoS}_{2}$ and the $\mathrm{Au}$ surface is relatively small, it is not surprising to see that the band structure of adsorbed $\mathrm{MoS}_{2}$ resembles that of free-standing $\mathrm{MoS}_{2}$, shown in Fig. 2.3(a). Note that in the $\sqrt{3} \times \sqrt{3} \mathrm{MoS}_{2}$ cell the bands are folded such that the direct band gap appears at the $\Gamma$ point. The work function of clean $\mathrm{Au}(111)$ is changed by only $0.08 \mathrm{eV}$ by the $4.2 \%$ compression of its lattice.

For comparison, Fig. 2.3(c) shows the band structure of $\mathrm{MoS}_{2} / \mathrm{Au}(111)$ when $\mathrm{MoS}_{2}$ is stretched by $4.2 \%$ to match the $\mathrm{Au}(111)$ lattice. Clearly the band structure of $\mathrm{MoS}_{2}$ is now changed significantly. It no longer shows a direct band gap at $\Gamma$, but an indirect band gap, and the size of the band gap is reduced to $\sim 1 \mathrm{eV}$, which is consistent with previous studies [101, 102, 103, 104, 105, 106]. The Schottky barrier for electrons (the energy difference between the bottom of the conduction band and the Fermi level), which is a sizable $0.7 \mathrm{eV}$ in Fig. 2.3(b), is reduced to zero in 
Table 2.2: In-plane supercell defined by the $\operatorname{MoS}_{2}$ lattice vector $R(\alpha) \vec{T}_{1}$, where $\vec{T}_{1}=$ $n_{1} \vec{a}_{1}+n_{2} \vec{a}_{2}$ and the metal lattice vector $\vec{T}_{1}^{\prime}=m_{1} \vec{b}_{1}+m_{2} \vec{b}_{2} . \delta$ gives the mismatch between the $\mathrm{MoS}_{2}$ and metal lattices, Eq. 2.7 (PBE values).

\begin{tabular}{|c|c|c|c|c|}
\hline & $n_{1}, n_{2}$ & $m_{1}, m_{2}$ & $\alpha$ & $\delta(\%)$ \\
\hline $\mathrm{Mg}$ & 1,0 & 1,0 & $0^{\circ}$ & 0.6 \\
$\mathrm{Al}$ & $4,-1$ & 4,0 & $13.9^{\circ}$ & 0.5 \\
$\mathrm{Ag}$ & $4,-1$ & 4,0 & $13.9^{\circ}$ & 0.15 \\
$\mathrm{Ti}$ & $5,-2$ & 4,0 & $23.4^{\mathrm{o}}$ & 0.7 \\
$\mathrm{Cu}$ & 4,0 & 5,0 & $0^{\circ}$ & 0.3 \\
$\mathrm{Au}$ & $4,-1$ & 4,0 & $13.9^{\circ}$ & 0.15 \\
$\mathrm{Pd}$ & 1,1 & 2,0 & $30^{\circ}$ & 0.3 \\
$\mathrm{Pt}$ & 1,1 & 2,0 & $30^{\circ}$ & 0.3 \\
$\mathrm{Co}$ & $5,-4$ & $4,-3$ & $3^{\mathrm{o}}$ & 0.01 \\
$\mathrm{Ni}$ & $5,-4$ & $4,-3$ & $3^{\mathrm{o}}$ & 0.8 \\
\hline
\end{tabular}

Fig. 2.3(c) as in Ref. [81]. The latter is clearly unphysical: one would not expect a high work-function metal such as Au to form a barrierless contact for electrons. Indeed experimentally $\mathrm{Au}$ is found to form a substantial Schottky barrier with $\mathrm{MoS}_{2}$ [71, 74, 75, 107, 108].

In the following we base the in-plane lattice constant of the $\mathrm{MoS}_{2} /$ metal slab on the optimized values of free-standing $\mathrm{MoS}_{2}$, which are 3.13, 3.18, and $3.19 \AA$ for the LDA, optb88-vdW-DF, and PBE functionals, respectively. Experimentally reported bulk $\mathrm{MoS}_{2}$ lattice constants are in the range 3.13-3.16 $\AA$ [109, 110, 111], suggesting that the LDA result may be more accurate and both PBE and the vdW functional are overestimating the lattice constant somewhat.

In making a commensurable structure we adapt the metal to the $\mathrm{MoS}_{2}$ lattice. To minimize the artificial strain that is introduced by this adaptation, we construct inplane supercells following the procedure of Ref. [112]. We denote a basis vector of a $\mathrm{MoS}_{2}$ supercell by $\vec{T}_{1}=n_{1} \vec{a}_{1}+n_{2} \vec{a}_{2}$, with $\left\{\vec{a}_{1}, \vec{a}_{2}\right\}$ the basis vectors of the primitive cell, and $n_{1}, n_{2}$ integers. Similarly, $\vec{T}_{1}^{\prime}=m_{1} \vec{b}_{1}+m_{2} \vec{b}_{2}$ is a basis vector of a metal surface supercell, with $\left\{\vec{b}_{1}, \vec{b}_{2}\right\}$ the basis vectors of the primitive cell, and $m_{1}, m_{2}$ integers. We search for a set of values for $n_{1}, n_{2}, m_{1}$, and $m_{2}$, such that the difference between the $\mathrm{MoS}_{2}$ and the metal supercell basis vectors is less than a margin $\delta$,

$$
\frac{\left|\vec{T}_{1}\right|-\left|\vec{T}_{1}^{\prime}\right|}{\left|\vec{T}_{1}\right|} \leq \delta
$$

We then rotate the $\mathrm{MoS}_{2}$ lattice by an angle $\alpha$ such, that the directions of the $\vec{T}_{1}$ and $\vec{T}_{1}^{\prime}$ vectors coincide. Because of the symmetry of the lattice the second basis vector of the supercell is easily obtained by a $120^{\circ}$ rotation, $\vec{T}_{2}=R\left(120^{\circ}\right) \vec{T}_{1}$. The commonly used surface science notation of this supercell is a $\sqrt{N} \times \sqrt{N} R \alpha \operatorname{MoS}_{2}$ lattice on top of a $\sqrt{M} \times \sqrt{M}$ metal lattice, where $N=n_{1}^{2}+n_{2}^{2}+n_{1} n_{2}$ and $M=m_{1}^{2}+m_{2}^{2}+m_{1} m_{2}$.

The parameter $\delta$ determines the mismatch between the $\mathrm{MoS}_{2}$ and the metal lat- 


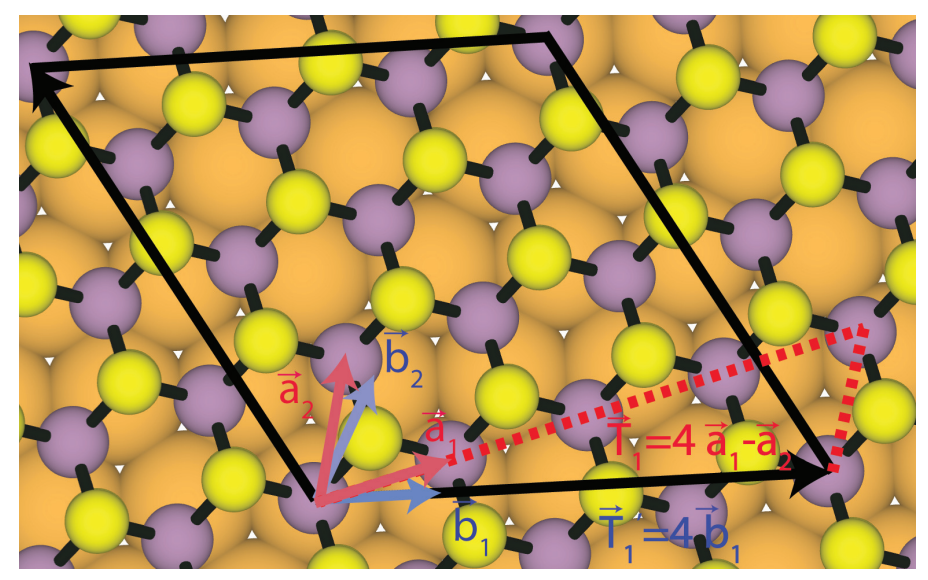

Figure 2.4: (Color online) Top view of $\mathrm{MoS}_{2} / \mathrm{Au}(111)$ interface indicating the supercell (black lines), the primitive basis vectors $\vec{a}_{1}, \vec{a}_{2}$ and $\vec{b}_{1}, \vec{b}_{2}$ of the $\operatorname{MoS}_{2}$ and $\mathrm{Au}(111)$ lattices, respectively, and the basis vector $\vec{T}_{1}$ and $\vec{T}_{1}^{\prime}$ of the supercell.

Table 2.3: Equilibrium bonding distance $d_{e q}$, binding energy $E_{b}$, and interface potential step $\Delta V$, for $\mathrm{MoS}_{2}$ on metal (111) surfaces, calculated with supercell lattices with a different mismatch $\delta$.

\begin{tabular}{|c||ccc|ccc|}
\hline \multirow{2}{*}{$\delta(\%)$} & \multicolumn{4}{|c}{$\mathrm{Au}$} & \multicolumn{4}{c}{$\mathrm{Ag}$} \\
\cline { 2 - 7 } & $d_{e q}(\AA)$ & $\Delta V(e V)$ & $E_{b}(e V)$ & $d_{e q}(\AA)$ & $\Delta V(e V)$ & $E_{b}$ \\
\hline 0.15 & 2.9 & 0.41 & -0.33 & 2.8 & 0.11 & -0.35 \\
4.2 & 3.1 & 0.51 & -0.30 & 2.9 & 0.47 & -0.32 \\
\hline
\end{tabular}

tices, and the strain we apply to the metal lattice. In this study, we choose the smallest supercell for which $\delta<1 \%$. Figure 2.4 gives an example of a supercell for $\mathrm{MoS}_{2}$ on $\mathrm{Au}(111)$ that is constructed this way, and Table 2.2 lists the supercells and the lattice mismatch $\delta$ used in this study for the different metals.

In the calculations discussed in Secs. 2.2.2 and 2.2.3 we have used a $\sqrt{3} \times \sqrt{3} R 30^{\circ}$ $\mathrm{MoS}_{2}$ cell on top of a $2 \times 2 \mathrm{Au}(111)$ cell, which leads to a lattice mismatch of $4.2 \%$. A $\sqrt{13} \times \sqrt{13} R 13.9^{\circ}$ on top of a $4 \times 4$ Au supercell, see Table 2.2 and Fig. 2.4, reduces the lattice mismatch to $0.15 \%$. Figure 2.5 shows that the binding energy curves for the two structures are quite similar. The equilibrium binding energy is increased by $0.03 \mathrm{eV}$ upon compressing the Au lattice by $4.2 \%$, and the equilibrium binding distance is decreased by $0.02 \AA$. Typically the interface potential step is affected by the compression on a scale of $0.1 \mathrm{eV}$, as is shown in Table 2.3. However sometimes the effect is larger, as for Ag. In conclusion, compressing the metal lattice does not generally have the same dramatic effect as stretching the $\mathrm{MoS}_{2}$ lattice has, but large lattice mismatches should be avoided. 


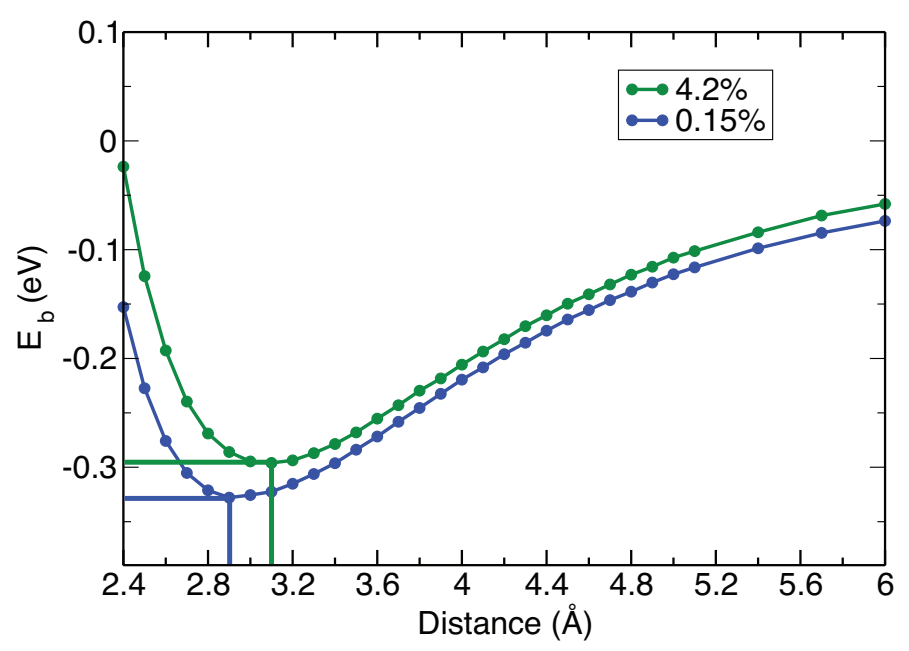

Figure 2.5: (Color online) Binding energy curves $E_{b}(d)(\mathrm{eV})$ of $\mathrm{MoS}_{2}$ on $\mathrm{Au}(111)$, calculated with opt8b-vdW-DF functional for a mismatch of $0.15 \%$ (blue), and $4.2 \%$ (green) between the $\mathrm{MoS}_{2}$ and the $\mathrm{Au}(111)$ lattices.

\subsection{Results}

\subsubsection{Metal/MoS $\mathrm{M}_{2}$ interaction}

Calculated equilibrium binding energies and bonding distances for the $\mathrm{MoS}_{2} /$ metal structures of Table 2.2 are listed in Table 2.4. The binding energies obtained with opt88-vdW-DF are in the range -0.3 to $-0.6 \mathrm{eV}$. These numbers seem somewhat too low in order to classify the bonding as physisorption, yet too high to call it chemisorption. Van der Waals interactions play an important role in the bonding, which becomes especially clear when comparing to the results obtained by PBE. The PBE functional lacks van der Waals interactions, and it typically captures only approximately half the $\mathrm{MoS}_{2} /$ metal binding energy or less.

A noticeable exception is $\mathrm{MoS}_{2} / \mathrm{Ti}(0001)$, where PBE gives approximately double the opt88-vdW-DF binding energy. It suggests that $\mathrm{MoS}_{2}$ is chemisorbed on $\mathrm{Ti}(0001)$, which is described better by PBE. This case will be discussed in more detail in Sec. 2.3.3. In contrast, the PBE functional essentially fails to give bonding for the adsorption of $\mathrm{MoS}_{2}$ on $\mathrm{Au}(111)$, and all bonding comes from van der Waals interactions, so we may classify this case as physisorption. For the other metals it is difficult to make a distinction between physisorption and chemisorption on the basis of the binding energy alone.

In general terms, physisorption is accompanied by a weak perturbation of the electronic structure of the adsorbed layer, whereas chemisorption results in a sizable perturbation of that electronic structure. For graphene and $h$-BN adsorbed on metal 
Table 2.4: Equilibrium binding energy $E_{b}$, and bonding distance $d_{e q}$, for $\mathrm{MoS}_{2}$ on metal (111) and (0001) surfaces in the interface structures of Table 2.2, calculated with the optb88b-vdW-DF and the PBE functionals.

\begin{tabular}{|c|c|c|c|c|}
\hline & $\begin{array}{c}E_{\mathrm{b}(\mathrm{vdW})} \\
(\mathrm{eV})\end{array}$ & $\begin{array}{c}d_{\mathrm{eq}(\mathrm{vdw})} \\
(\AA)\end{array}$ & $\begin{array}{c}E_{\mathrm{b}(\mathrm{PBE})} \\
(\mathrm{eV})\end{array}$ & $\begin{array}{c}d_{\mathrm{eq}(\mathrm{PBE})} \\
(\AA)\end{array}$ \\
\hline $\mathrm{Mg}$ & -0.55 & 2.3 & -0.20 & 2.2 \\
\hline $\mathrm{Al}$ & -0.30 & 2.8 & -0.30 & 2.8 \\
\hline $\mathrm{Ag}$ & -0.35 & 2.8 & -0.08 & 2.9 \\
\hline $\mathrm{Ti}$ & -0.51 & 2.3 & -0.67 & 2.3 \\
\hline $\mathrm{Cu}$ & -0.40 & 2.5 & -0.16 & 2.4 \\
\hline $\mathrm{Au}$ & -0.33 & 2.9 & -0.02 & 3.3 \\
\hline $\mathrm{Pd}$ & -0.54 & 2.4 & -0.25 & 2.3 \\
\hline $\mathrm{Ni}$ & -0.51 & 2.2 & -0.25 & 2.2 \\
\hline Co & -0.57 & 2.2 & -0.29 & 2.2 \\
\hline $\mathrm{Pt}$ & -0.43 & 2.6 & -0.11 & 2.6 \\
\hline
\end{tabular}

surfaces it is possible to correlate that perturbation with the equilibrium bonding distances $d_{\text {eq }}$. Those distances can be divided into two groups separated by a critical binding distance $d_{\mathrm{c}}$. For $d_{\mathrm{eq}}>d_{\mathrm{c}}$, the bonding is physisorption, and for $d_{\mathrm{eq}}<d_{\mathrm{c}}$, the bonding is chemisorption. For graphene and $h$-BN this distinction is successful because there are hardly any cases where $d_{\text {eq }} \approx d_{\mathrm{c}} \approx 2.8 \AA$ as is illustrated in Fig. 2.6. Clearly bonding distances and energies are correlated; a shorter distance generally gives a lower energy.

Plotting the binding energies and distances for $\mathrm{MoS}_{2} /$ metal interfaces in Fig. 2.6, one observes that the distinction between physisorption and chemisorption is much less clear for this case. The binding of $\mathrm{MoS}_{2}$ to a metal substrate is stronger than that of graphene or $h$-BN, reflecting the fact that van der Waals interactions increase with the atomic number. Maybe somewhat surprisingly the bonding distance of $\mathrm{MoS}_{2}$ to a metal substrate is generally shorter than that of graphene or $h$-BN. Graphene and $h$-BN have $\pi$-orbitals that stick out below their respective planes, which give rise to a substantial Pauli repulsion at distances to the metal plane of $\lesssim 3 \AA$ [40]. Apparently the wave functions of $\mathrm{MoS}_{2}$ do not stick out that far below the plane of the bottom sulfur layer.

The bonding distances for $\mathrm{MoS}_{2} /$ metal interfaces cannot easily be simply into two groups, as is the case for graphene and $h$-BN/metal interfaces. Instead there is a more gradual scale. The bonding distances of $\mathrm{MoS}_{2}$ on $\mathrm{Al}, \mathrm{Au}$ and $\mathrm{Ag}$ are on the physisorption side of Fig. 2.6, whereas on $\mathrm{Co}, \mathrm{Ni}, \mathrm{Mg}$, and $\mathrm{Ti}$, they are more on the chemisorption side, with $\mathrm{Pt}, \mathrm{Cu}, \mathrm{Pd}$ as intermediate cases. However, a clear dividing line like for graphene and $h$-BN can not be drawn. Indeed if one considers the $\mathrm{MoS}_{2} /$ metal interface for two similar metals that give rise to a fairly large difference in bonding distance and binding energy: $\mathrm{Ag}$ and $\mathrm{Pd}$, one does not observe a qualitative difference in the the electronic structure of the $\mathrm{MoS}_{2}$ adsorbate, see Fig. 2.7. In both cases the $\mathrm{MoS}_{2}$ bands are perturbed by the metal-MoS $\mathrm{S}_{2}$ interaction, but the 


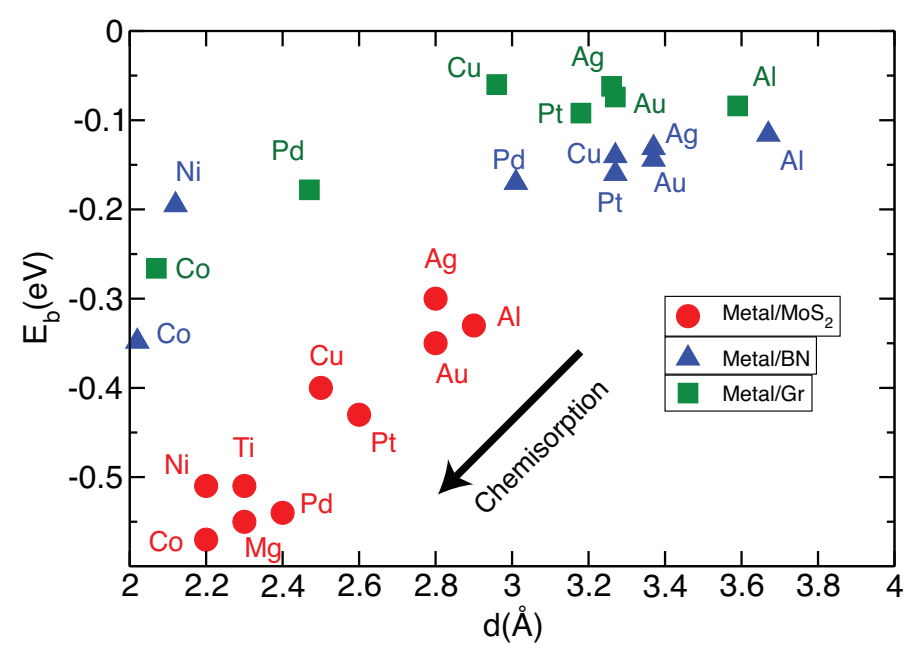

Figure 2.6: (Color online) Binding energy $E_{b}(\mathrm{eV})$ per $\mathrm{MoS}_{2}$ versus equilibrium bonding distance $d_{\text {eq }}(\AA)$ for $\mathrm{MoS}_{2}$ adsorbed on metal(111) and (0001) substrates (red circles), as calculated with the optb88-vdW-DF functional. For comparison, results for $h$-BN (blue triangles) [78], and graphene (green squares) [113], are also shown.

signature of the $\mathrm{MoS}_{2}$ bands can still be recognized. In particular, it still seems to be possible to identify the top of the $\mathrm{MoS}_{2}$ valence band, and the bottom of the conduction band. Nevertheless the $\mathrm{MoS}_{2}$ states do hybridize with those of the metal substrate, as we will discuss in the next section.

Another way of characterizing the bonding is to analyze the geometry of the $\mathrm{MoS}_{2} /$ metal interface. Chemisorption involves the formation of chemical bonds between the adsorbate and the metal, which frequently also leads to a deformation of the adsorbate's structure. We can define a displacement $\Delta_{i}=\left|\mathbf{R}_{\mathbf{i}}-\mathbf{R}_{0, i}\right|$ between the position $\mathbf{R}_{\mathbf{i}}$ of an atom $i$ in the optimized $\mathrm{MoS}_{2} /$ metal structure, and its position $\mathbf{R}_{0, i}$ in the free-standing $\mathrm{MoS}_{2}$ or in the clean metal substrate. The displacements are obtained in a two-step procedure. First the $\mathrm{MoS}_{2} /$ metal structure is optimized while freezing the $\mathrm{MoS}_{2}$ layer and the metal substrate in their free-standing geometries. Once the equilibrium distance $d_{\mathrm{eq}}$ is obtained, as in Figs. 2.2 and 2.5, all atomic positions are relaxed, and this last step defines the displacements. Table 2.4 gives $\bar{\Delta}_{\mathrm{S}}$ and $\bar{\Delta}_{\mathrm{M}}$, which are the average displacements of the bottom layer of sulfur atoms, and of the top layer of metal atoms, respectively, for some representative metal substrates. In addition, this table gives the maximum displacements $\Delta_{\mathrm{S}}^{\max }$ and $\Delta_{\mathrm{M}}^{\max }$.

The displacements are quite large for the $\mathrm{MoS}_{2} / \mathrm{Ti}(0001)$ structure, indicating that there is a significant distortion of the geometries of both the $\mathrm{MoS}_{2}$ adsorbate and the Ti surface, which strongly suggests that $\mathrm{MoS}_{2}$ is chemisorbed on Ti. At the opposite end of the scale we find $\mathrm{MoS}_{2} / \mathrm{Au}(111)$, where the atomic displacements are small, indicating that here we are in the physisorption regime. The behavior of the other metal substrates is in between these two extreme cases but more to the physisorption 

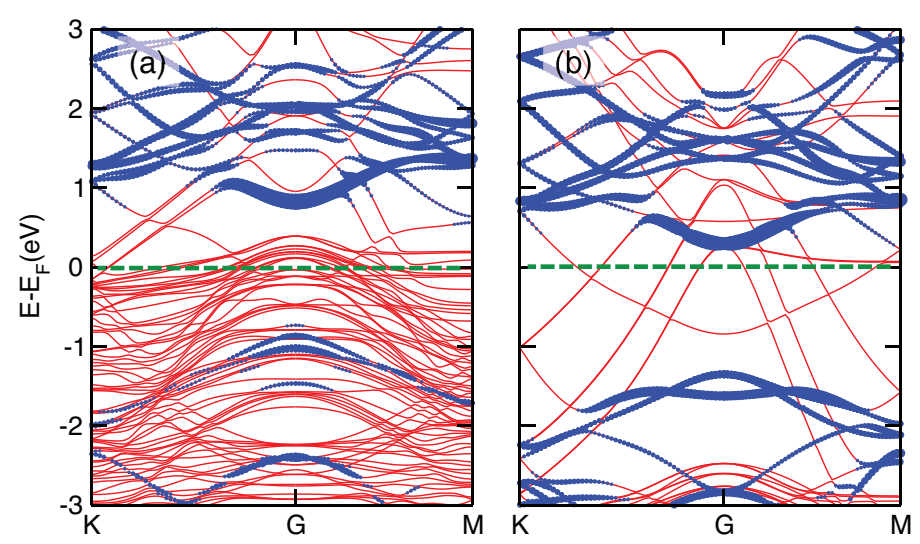

Figure 2.7: (Color online) (a) Band structure of $\mathrm{MoS}_{2} / \mathrm{Pd}(111)$; the blue color indicates the weight of a projection of the wave functions on the $\mathrm{MoS}_{2}$ sites; (b) idem for $\mathrm{MoS}_{2} / \mathrm{Ag}(111)$; for comparison both band structures are shown in a $\sqrt{3} \times \sqrt{3}$ surface cell.

side. The $3 \mathrm{~d}$ transition metals $\mathrm{Co}, \mathrm{Ni}, \mathrm{Cu}$ and the low work function simple metal $\mathrm{Mg}$ show somewhat larger distortions than the $4 \mathrm{~d}$ and $5 \mathrm{~d}$ metals $\mathrm{Pd}, \mathrm{Ag}, \mathrm{Pt}$ and the simple metal Al.

\subsubsection{Interface Potential Step and Schottky Barrier}

Table 2.6 gives the interface potential steps $\Delta V$ created by the adsorption of $\mathrm{MoS}_{2}$ on a metal substrate. This potential step strongly influences the Schottky barrier at metal/MoS 2 contacts, see Eq. 3.1, and as such it plays an important role in the physics of $\mathrm{MoS}_{2}$ semiconductor devices. The potential steps can be divided into two groups, i.e., positive $\Delta V$ for metals with a high work function, and negative $\Delta V$ for low work function metals. A positive $\Delta V$ means that adsorption of $\mathrm{MoS}_{2}$ effectively lowers the work function of the substrate. The $\mathrm{MoS}_{2}$ layer has no intrinsic dipole moment perpendicular to the layer that could create such a potential step. So the work function lowering is a purely electronic effect that results from the displacement of surface electron density into the metal by physisorption of the adsorbate.

This effect is known as the push-back effect or the pillow effect, which is a general phenomenon observed in the physisorption of closed-shell atoms, molecules, and layers on metal substrates. In Ref. [40] we have developed a quantitative model for this effect, based upon an anti-symmetrization of the product of the metal and adsorbate wave functions. When an adsorbate approaches a metal surface, the wave functions of the two systems overlap. Pauli exchange repulsion between these states leads to a spatial redistribution of the electron density, in particular to a decrease of the density in the overlap region. Since the metal wave functions are usually more extended and more easily deformable than those of the adsorbate, the net result of this redistribution is that electrons are pushed back into the metal, which effectively 
Table 2.5: The average displacements $\bar{\Delta}_{\mathrm{S}}$ and $\bar{\Delta}_{\mathrm{M}}$ of the bottom layer of sulfur atoms, and of the top layer of metal atoms, and the corresponding maximum displacements $\Delta_{\mathrm{S}}^{\max }$ and $\Delta_{\mathrm{M}}^{\max }$, after the adsorption.

\begin{tabular}{|c||c|c|c|c|}
\hline & $\bar{\Delta}_{\mathrm{S}}(\AA)$ & $\Delta_{\mathrm{S}}^{\max }(\AA)$ & $\bar{\Delta}_{\mathrm{M}}(\AA)$ & $\Delta_{\mathrm{M}}^{\max }(\AA)$ \\
\hline $\mathrm{Ti}$ & 0.101 & 0.152 & 0.176 & 0.483 \\
$\mathrm{Co}$ & 0.010 & 0.021 & 0.068 & 0.102 \\
$\mathrm{Pt}$ & 0.006 & 0.009 & 0.049 & 0.098 \\
$\mathrm{Ag}$ & 0.002 & 0.005 & 0.030 & 0.058 \\
$\mathrm{Au}$ & 0.001 & 0.004 & 0.019 & 0.044 \\
$\mathrm{Pd}$ & 0.002 & 0.003 & 0.026 & 0.051 \\
$\mathrm{Ni}$ & 0.017 & 0.034 & 0.089 & 0.150 \\
$\mathrm{Al}$ & 0.001 & 0.047 & 0.046 & 0.105 \\
$\mathrm{Mg}$ & 0.022 & 0.022 & 0.091 & 0.091 \\
$\mathrm{Cu}$ & 0.033 & 0.052 & 0.070 & 0.124 \\
\hline
\end{tabular}

lowers the work function.

In the adsorption of graphene and $h$-BN on high work function metals we found potentials steps of up to 1-2 eV. The potential steps for $\mathrm{MoS}_{2}$ adsorbed on the same metals are generally smaller, and more typically around $0.3-0.4 \mathrm{eV}$. The wave functions of first-row elements $(\mathrm{B}, \mathrm{C}, \mathrm{N})$ are compact and not easily deformable, as compared to the wave functions of the metal substrate. The effect of Pauli repulsion in the metal/adsorbate overlap region is then very asymmetric. It is foremost the metal electron density that is deformed, i.e. pushed back, which gives a large work function lowering. If the adsorbate contains heavier elements, such as $\mathrm{MoS}_{2}$, the effect of Pauli repulsion is more symmetric, i.e., both the metal and the adsorbate electrons are pushed out of the overlap region in a more symmetric way. This gives a smaller effect on the work function. Note that if the effect of Pauli repulsion would be completely symmetric, the work function would be unchanged.

Low work function metals experience an increase of the work function upon adsorption of $\mathrm{MoS}_{2}$, i.e. a negative $\Delta V$, which indicates a net transfer of electrons from the metal to the $\mathrm{MoS}_{2}$ adsorbate. As $\mathrm{MoS}_{2}$ is a semiconductor it can only receive electrons in its conduction band. Therefore, for low work function metal substrates one expects the Fermi level to be in the conduction band of $\mathrm{MoS}_{2}$. Analysis of the electronic structure of the $\mathrm{MoS}_{2} /$ metal slab however shows that this is not the case. The interaction between $\mathrm{MoS}_{2}$ and the metal at the interface leads to interface states with energies in the $\mathrm{MoS}_{2}$ band gap. That seems obvious if $\mathrm{MoS}_{2}$ is chemisorbed onto the substrate, as in the case of $\mathrm{MoS}_{2} / \mathrm{Ti}(0001)$, which we will discuss in the next section.

Somewhat surprisingly, a significant density of interface states also forms if the interaction between $\mathrm{MoS}_{2}$ and the metal substrate is relatively weak. For example, as discussed in the previous section, the interaction between $\mathrm{MoS}_{2}$ and $\mathrm{Al}(111)$ can be classified as physisorption, see Fig. 2.6. Nevertheless, states with energies inside the $\mathrm{MoS}_{2}$ band gap are formed at the $\mathrm{MoS}_{2} / \mathrm{Al}(111)$ interface, as is immedi- 
Table 2.6: Metal work function $W_{\mathrm{M}}$, interface potential step $\Delta V$, and Schottky barrier height $\Phi_{\mathrm{n}}$ calculated with the PBE and opt88-vdW-DF functionals, with calculated $\mathrm{MoS}_{2}$ electron affinities of $\chi=4.30 \mathrm{eV}$ and $\chi=4.57 \mathrm{eV}$, respectively.

\begin{tabular}{|c|c|c|c|c|c|c|}
\hline & \multicolumn{2}{|c|}{ PBE } & \multicolumn{4}{|c|}{ vdW-DF } \\
\hline & $W_{\mathrm{M}}(e V)$ & $\Delta V(e V)$ & $\Phi_{\mathrm{n}}(e V)$ & $W_{M}(e V)$ & $\Delta V(e V)$ & $\Phi_{\mathrm{n}}(e V)$ \\
\hline $\mathrm{Mg}$ & 3.78 & -0.77 & 0.25 & 3.96 & -0.74 & 0.13 \\
\hline $\mathrm{Al}$ & 4.00 & -0.54 & 0.24 & 4.20 & -0.56 & 0.19 \\
\hline $\mathrm{Ag}$ & 4.47 & 0.10 & 0.07 & 4.82 & 0.11 & 0.14 \\
\hline $\mathrm{Ti}$ & 4.52 & -0.28 & $0.53^{a}$ & 4.80 & -0.27 & $0.54^{\mathrm{a}}$ \\
\hline $\mathrm{Cu}$ & 4.70 & 0.35 & 0.05 & 5.10 & 0.39 & 0.14 \\
\hline $\mathrm{Au}$ & 5.30 & 0.32 & 0.68 & 5.58 & 0.41 & 0.60 \\
\hline $\mathrm{Pd}$ & 5.35 & 0.35 & 0.70 & 5.48 & 0.30 & 0.61 \\
\hline $\mathrm{Pt}$ & 5.75 & 0.64 & 0.81 & 5.96 & 0.68 & 0.71 \\
\hline $\mathrm{Co}$ & 5.13 & 0.29 & 0.54 & 5.42 & 0.34 & 0.51 \\
\hline $\mathrm{Ni}$ & 5.17 & 0.28 & 0.59 & 5.40 & 0.37 & 0.46 \\
\hline
\end{tabular}

a See Sec. 2.3.3.

ately obvious when comparing the density of states of the interface with that of freestanding $\mathrm{MoS}_{2}$, see Fig. 2.8. The density of these interface states is not extremely high, yet sufficiently high to pin the Fermi level below the $\mathrm{MoS}_{2}$ conduction band, as demonstrated by Fig. 2.8. The density of interface states increases with increasing $\mathrm{MoS}_{2} /$ metal interaction, but even for physisorption it seems sufficiently high to prevent the Fermi level from reaching the $\mathrm{MoS}_{2}$ conduction band.

One can prove that these interface states are indeed responsible for pinning the Fermi level by artificially enlarging the distance between the $\mathrm{MoS}_{2}$ layer and the metal surface. This breaks the direct $\mathrm{MoS}_{2} /$ metal contact that is responsible for the formation of interface states. In the absence of interface states, the Fermi level is at the bottom of the $\mathrm{MoS}_{2}$ conduction band, see Fig. 2.9(d), which is what one would expect if the work function of the metal $W_{\mathrm{M}}$ is smaller than the electron affinity of $\mathrm{MoS}_{2} \chi_{\mathrm{MoS}_{2}}$, see Eq. 3.1. A transfer of electrons between the metal and the $\mathrm{MoS}_{2}$ overlayer then yields a charge distribution that can be associated with a simple interface dipole, see Fig. 2.9(b). In contrast, if interface states are formed, the band structure of adsorbed $\mathrm{MoS}_{2}$ is perturbed, see Fig. 2.9(c), and the pattern of the charge distribution at the interface is much more complicated, as shown in Fig. 2.9(a). In that case, the interface states pin the Fermi level below the bottom of the $\mathrm{MoS}_{2}$ conduction band, see Fig. 2.8.

Schottky barrier heights (SBHs) for electrons, calculated according to Eq. 3.1, are also listed in Table 2.6. The functional causes some uncertainty, as the work functions of the clean metal surfaces obtained with the opt88-vdW-DF functional tend to be somewhat higher than those obtained with the PBE functional. LDA in general gives even higher work functions, so opt88-vdW-DF gives work functions that are in between those of PBE and LDA $[78,88]$. Note that the interface potential steps 


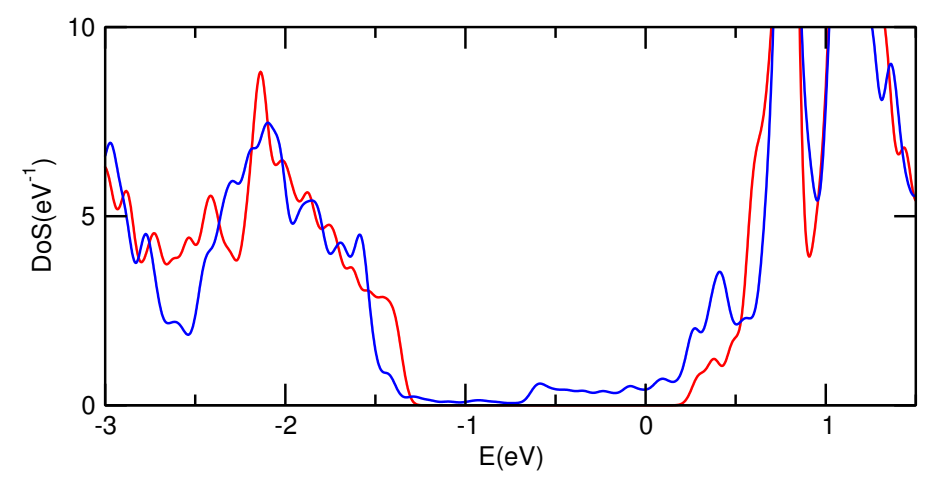

Figure 2.8: (Color online) (blue) The total density of states of the $\mathrm{MoS}_{2} / \mathrm{Al}(111)$ slab; (red) the sum of the densities of states of free-standing $\mathrm{MoS}_{2}$ and of the $\mathrm{Al}(111)$ slab. The densities of states are aligned by aligning the Mo $4 \mathrm{~s}$ core levels, and the Al 2p core levels.

$\Delta V$ do not depend strongly on the functional. As the opt88-vdW-DF functional also gives a larger electron affinity for $\mathrm{MoS}_{2}$, the Schottky barrier $\Phi_{\mathrm{n}}$ according to Eq. 3.1, also does not depend strongly on the functional.

Nevertheless there is an uncertainty in the calculated SBHs coming from the $\mathrm{MoS}_{2}$ electron affinity. One may argue that the electron affinity calculated with either of the functionals is too high, as DFT band gaps are too small, which would give SBHs that are too small. The band gaps of a $\mathrm{MoS}_{2}$ monolayer calculated with the PBE and opt88-vdW-DF functionals are $1.63 \mathrm{eV}$ and $1.67 \mathrm{eV}$, respectively, which can be compared to the experimental optical band gap of $1.86 \mathrm{eV}[25,114]$. Such a comparison is not stricktly fair, as the difference should reflect the exciton binding energy. Exciton binding energies between negligible [25], and $0.5 \mathrm{eV}$ [115], have been reported. Note however that the exciton binding energy strongly depends on screening by the environment $[115,116]$. For $\mathrm{MoS}_{2}$ adsorbed on metals one expects the screening to be large, and consequently the exciton binding energy to be relatively small.

We see that the SBH decreases with decreasing metal work function but does not go to zero. Instead it goes through a minimum for $\mathrm{Cu}$ and $\mathrm{Ag}$, and then increases again for low work function metals like $\mathrm{Al}$ and $\mathrm{Mg}$. As discussed above, this phenomenon is caused by interface states. The only way to get rid of such states is to break the direct interaction between $\mathrm{MoS}_{2}$ and the metal substrate. Ref. [77] discusses a practical way of doing this by inserting an atomic layer between the metal surface and the $\mathrm{MoS}_{2}$ layer. If this intermediate layer is purely van der Waals-bonded to $\mathrm{MoS}_{2}$, no gap states are formed at its interface with $\mathrm{MoS}_{2}$. In addition the intermediate layer should be transparent to electrons, such that the interface resistance is not dramatically increased. A monolayer of $h$-BN or graphene satisfy these criteria [77, 117, 118]. 

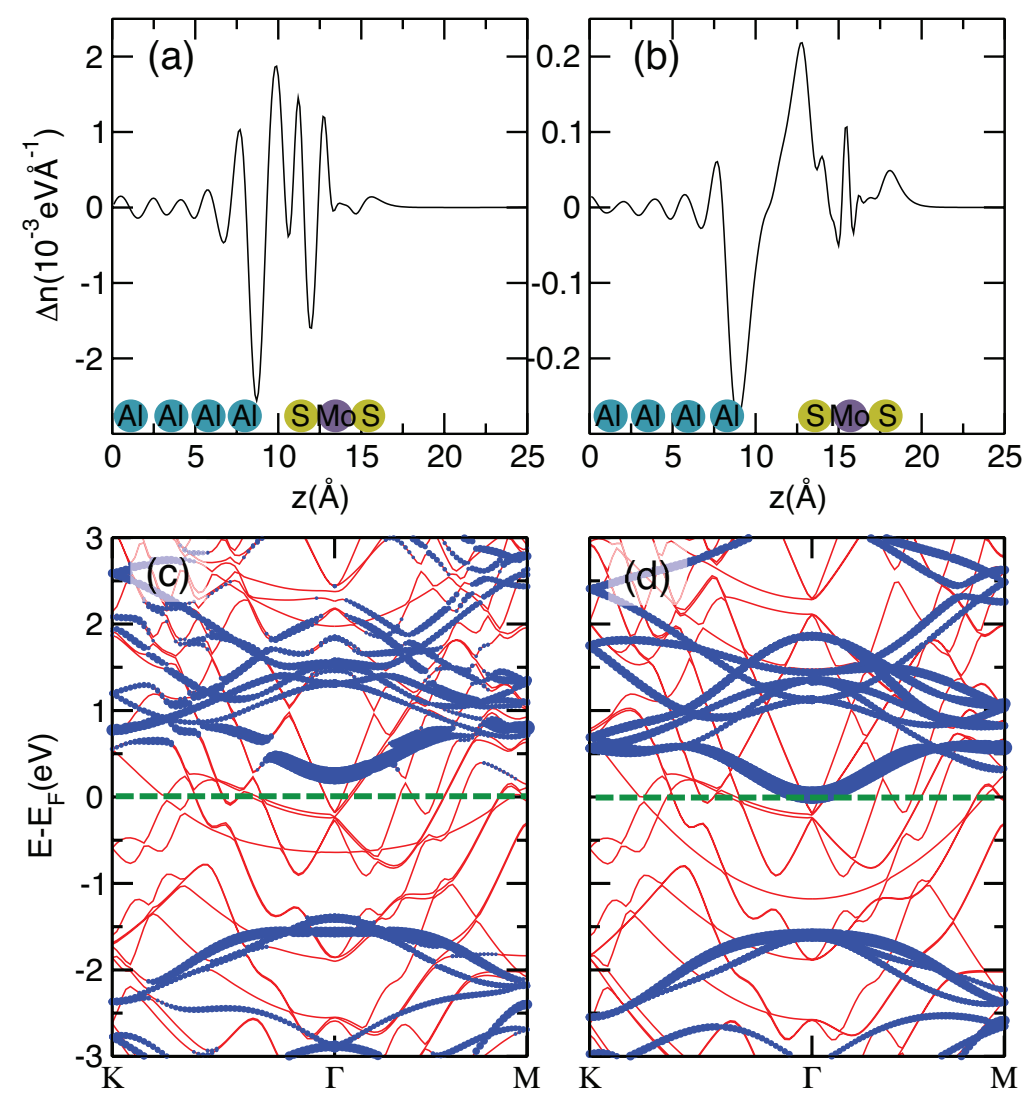

Figure 2.9: (Color online) (a) The electron density difference $\Delta n(z)$ of the $\mathrm{MoS}_{2} / \mathrm{Al}(111)$ interface at the equilibrium distance $d_{e q}=3.2 \AA$, and (b) at a distance $d=6 \AA ;(\mathrm{c}, \mathrm{d})$ the corresponding band structures; the blue color indicates the weight of a projection of the wave functions on the $\mathrm{MoS}_{2}$ sites.

\subsection{3 $\mathrm{MoS}_{2} / \mathrm{Ti}(0001)$}

As discussed in Sec. 2.3.1, $\mathrm{MoS}_{2}$ is chemisorbed in Ti(0001). The binding energy and the equilibrium bonding distance of $\mathrm{MoS}_{2}$ on $\mathrm{Ti}(0001)$ does not seem to be qualitatively different from those for $\mathrm{MoS}_{2}$ on metal substrates such as $\mathrm{Co}(0001)$ or $\mathrm{Ni}(111)$, see Table 2.4 and Fig. 2.6. However, the structural deformation of the $\mathrm{MoS}_{2}$ layer adsorbed on $\mathrm{Ti}(0001)$ is much larger than that of $\mathrm{MoS}_{2}$ on other metals, see Table 2.5. This deformation is illustrated in Fig. 2.10. Atoms of the bottom sulfur layer make a bond with $\mathrm{Ti}$ atoms of the top layer of the substrate, where several of these metal atoms are pulled up from the substrate. The $\mathrm{MoS}_{2}$ and the $\mathrm{Ti}(0001)$ lattices do not fit very well; one needs a $\sqrt{19} \times \sqrt{19} R 23.4^{\circ} \mathrm{MoS}_{2}$ supercell on top of a $4 \times 4$ $\mathrm{Ti}(0001)$ supercell to get a mismatch below $1 \%$, see Table 2.2. The result therefore is a $\mathrm{MoS}_{2} / \mathrm{Ti}(0001)$ interface that contains a substantial local strain, which explains why the binding energy is not very large, despite the bonding being chemisorption. 


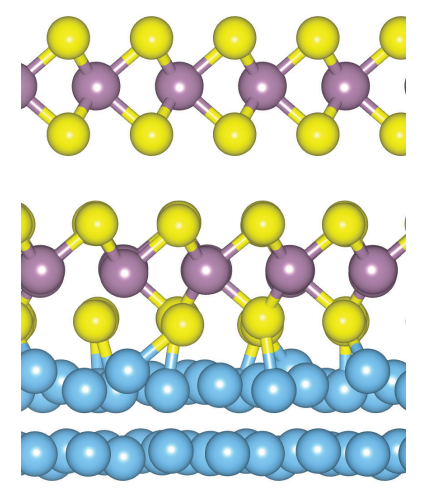

Figure 2.10: (Color online) Side view of two layers of $\mathrm{MoS}_{2}$ adsorbed on $\mathrm{Ti}(0001)$.

The potential step $\Delta V$ at the $\mathrm{MoS}_{2} / \mathrm{Ti}(0001)$ interface is negative, in contrast to the potential step at the $\mathrm{MoS}_{2} / \mathrm{Ag}(111)$ interface for instance, which is positive, despite the fact that the work functions of Ti and Ag are very similar, see Table 2.6. We argued that physisorption should lead to a positive potential step because of the Pauli repulsion effect, and indeed $\mathrm{MoS}_{2}$ is physisorbed on $\mathrm{Ag}(111)$. Chemisorption, as in the case of $\mathrm{MoS}_{2}$ on $\mathrm{Ti}(0001)$, leads to a more drastic reorganization of the charge distribution at the interface, because of the formation of new chemical bonds. Upon the formation of these bonds there is apparently a net displacement of electronic density towards the sulfur atoms, which is not unreasonable as sulfur is more electronegative than Ti. This displacement results in an increase of the work function, i.e., a negative $\Delta V$.

One expects that chemisorption also leads to a strong perturbation of the electronic structure of the adsorbate. Figure 2.11 shows the density of states (DOS) of a $\mathrm{MoS}_{2}$ bilayer projected on the individual $\mathrm{MoS}_{2}$ layers. The DOS of the first (chemisorbed) layer is indeed strongly perturbed as compared to the DOS of a freestanding $\mathrm{MoS}_{2}$ layer. The $\mathrm{MoS}_{2}$ wave functions strongly hybridize with those of the underlying Ti substrate, and the resulting hybridized states give a non-zero DOS for energies all through the $\mathrm{MoS}_{2}$ band gap. It is sometimes argued that such interface states promote having a good (ohmic) $\mathrm{MoS}_{2} /$ metal contact [81, 83]. One could however also argue that chemisorption is harmful to obtaining a good contact, because it damages the integrity of the $\mathrm{MoS}_{2}$ layer. In Ref. [119] it is found that $\mathrm{MoS}_{2} / \mathrm{Ag}$ gives a better contact than $\mathrm{MoS}_{2} / \mathrm{Ti}$, due to a much smoother interface in the former case, suggesting to prefer physisorption over chemisorption.

It is not possible to define a Schottky barrier for a single $\mathrm{MoS}_{2}$ layer adsorbed on $\mathrm{Ti}(0001)$. Chemisorption affects the electronic structure of $\mathrm{MoS}_{2}$ to such an extend that its semiconducting character is lost. It is however possible to define a Schottky barrier for a second $\mathrm{MoS}_{2}$ layer that is adsorbed on the first layer. The first and second $\mathrm{MoS}_{2}$ layer are bonded by a van der Waals interaction, which does not perturb the electronic structure of the second layer significantly. Indeed the DOS of the 


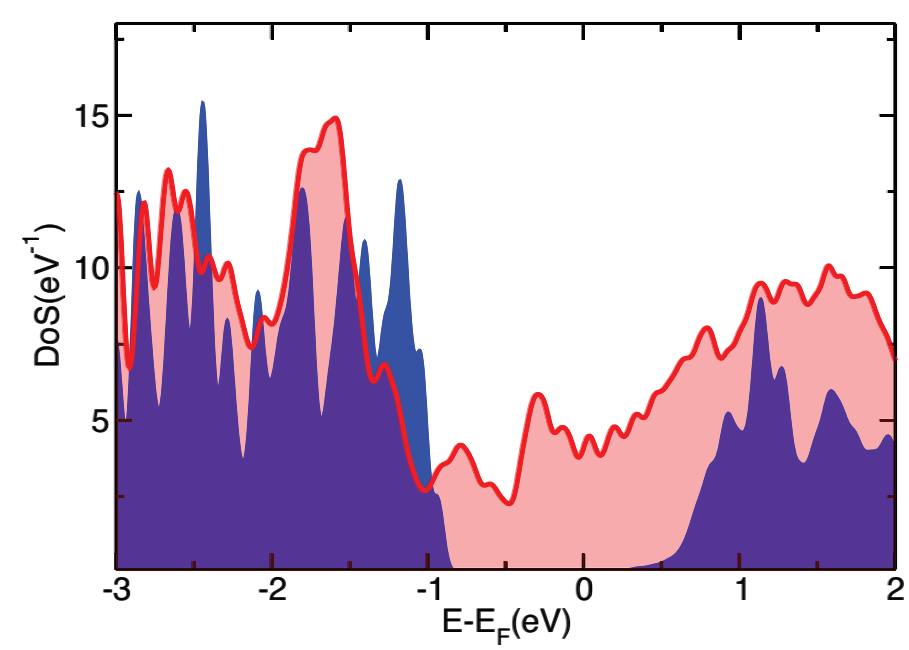

Figure 2.11: (Color online) The red and the blue shaded areas indicate the DOS projected on the first $\mathrm{MoS}_{2}$ layer of $\mathrm{MoS}_{2}$ adsorbed on $\mathrm{Ti}(0001)$ and on the second $\mathrm{MoS}_{2}$ layer, respectively.

second $\mathrm{MoS}_{2}$ layer is quite similar to that of a free-standing $\mathrm{MoS}_{2}$ layer with a clear band gap, see Fig. 2.11. The height of the Schottky barrier to the second layer is a sizable $0.54 \mathrm{eV}$. This mainly results from the fact that the first adsorbed $\mathrm{MoS}_{2}$ layer effectively increases the work function of the Ti substrate, see Table 2.6. The size of the Schottky barrier indicates that it is not possible to make an ohmic contact to pristine (undoped) $\mathrm{MoS}_{2}$ with Ti $[81,83]$.

\subsection{Summary and Conclusions}

In this paper, we explore the adsorption of $\mathrm{MoS}_{2}$ on a range of metal substrates by means of first-principles DFT calculations. The (111) surfaces of $\mathrm{Al}, \mathrm{Ni}, \mathrm{Cu}, \mathrm{Pd}, \mathrm{Ag}$, $\mathrm{Pt}$ and $\mathrm{Au}$, and the (0001) surfaces of $\mathrm{Mg}$, Co and Ti cover a range of metals with different interaction strengths, allowing for a systematic study of the metal-MoS interface.

We compare the results obtained with different DFT functionals. In many cases the GGA/PBE density functional only captures a small part of the binding energy of $\mathrm{MoS}_{2}$ on a metal substrate, as compared to the opt88-vdW-DF van der Waals density functional, which indicates the importance of van der Waals interactions in the interface bonding. Nevertheless, the equilibrium binding distances obtained with both functionals are generally very similar, and so are the interface potential steps and Schottky barrier heights. Exceptions are cases for which van der Waals interactions essentially describe the whole bonding, where PBE fails completely. LDA tends to overbind, leading to shorter binding distances and larger interface potential steps.

The interface structure that results from adsorbing an $\mathrm{MoS}_{2}$ layer on a metal sur- 
face will be incommensurable in most cases, as the two lattices have a mismatch. We investigate the effects of the artificial strain introduced by approximating the lattice using a commensurable supercell. We conclude that these effects are moderate provided the $\mathrm{MoS}_{2}$ lattice parameter is kept at its optimized value, and the metal lattice is strained. Large lattice mismatches should however be avoided, and straining the $\mathrm{MoS}_{2}$ lattice can lead to very unphysical results [81].

Of the metal substrates studied Ti is the one on which $\mathrm{MoS}_{2}$ is clearly chemisorbed. Adsorption of $\mathrm{MoS}_{2}$ on Ti(0001) is accompanied by a clear structural deformation of the Ti surface and of the $\mathrm{MoS}_{2}$ overlayer, due to the formation of bonds between the surface Ti atoms and the sulfur atoms at the interface. Formation of these interface bonds significantly alters the electronic structure of the $\mathrm{MoS}_{2}$ adsorbate. In particular, the interface states fill up the band gap of $\mathrm{MoS}_{2}$, which makes defining a Schottky barrier for this layer meaningless. However, for a second, unperturbed, adsorbed $\mathrm{MoS}_{2}$ layer a Schottky barrier of $0.54 \mathrm{eV}$ can be extracted.

$\mathrm{MoS}_{2}$ is physisorbed on $\mathrm{Au}(111)$, where the bonding is almost completely due to van der Waals interactions, and the structure and electronic structure of $\mathrm{MoS}_{2}$ are hardly perturbed by the adsorption. The properties of $\mathrm{MoS}_{2}$ adsorbed on other metal substrates fall in the range between the two extreme cases ( $\mathrm{Ti}$ and $\mathrm{Au}$ ), without the possibility of drawing a clear dividing line, as has been done for the adsorption of graphene or $h$-BN on metal substrates $[40,86,88,78]$.

Experiments have focused foremost on Schottky barrier heights. Transport measurements on multilayer $\mathrm{MoS}_{2}$ devices generally yield small numbers for the Schottky barrier heights, i.e. 0.03-0.2 eV, for different metals [120, 72, 71, 82, 75], whereas photoemission, photoconduction, and scanning tunneling spectroscopy give higher values $0.2-0.9 \mathrm{eV}[74,107,108,121]$. It has been suggested that the $\mathrm{MoS}_{2}$ samples used in devices is quite defective and inhomogeneous, such that the position of the Fermi level does not reflect an intrinsic property of $\mathrm{MoS}_{2}$ or of the $\mathrm{MoS}_{2} /$ metal contact [121,122], which obstructs a comparison to calculated results.

Our results for the $4 \mathrm{~d}$ and $5 \mathrm{~d}$ metals $\mathrm{Ag}, \mathrm{Au}, \mathrm{Pd}$, and Pt agree qualitatively with those reported in previous calculations [82, 83, 84, 85], provided the $\mathrm{MoS}_{2}$ lattice is not stretched [81]. Quantitatively, the reported Schottky barrier heights for these metals are $\sim 0.3 \mathrm{eV}$ larger than our results. These calculations were based upon the LDA functional, which tends to overbind, and to overestimate the metal work functions $[78,88]$. Compressing the metal lattice, which is sometimes required to accommodate a lattice mismatch in a small supercell, does not help either, as that gives an even higher work function [84]. The same is likely true for simple metals such as $\mathrm{Al}$ and In $[84,83]$. The strong interaction we find for Ti is also found in LDA calculations $[81,83,85]$. In those calculations the lattice mismatch used was large, however, which can alter the interface interactions.

The overall picture emerging from these calculations is that $\mathrm{MoS}_{2}$ interacts strongly with the early transition metals, where it is clearly chemisorbed. The interaction with the late transition metals is much weaker, where the $3 \mathrm{~d}$ metals interact stronger than the $4 \mathrm{~d}$ and $5 \mathrm{~d}$ metals. $\mathrm{MoS}_{2}$ interacts rather weakly with the simple metals, but 
the interaction increases for very low work function metals. In all but the strongly chemisorbed case, van der Waals forces play an important role in the interface interactions.

In case the interface interaction is weak (physisorption) the interface potential step can be understood as resulting from Pauli repulsion, which effectively decreases the substrate work function. The Schottky barrier is then simply calculated from the modified work function. Strong interaction (chemisorption) leads to the formation of bonds between the substrate metal atoms and the adsorbate sulfur atoms. It increases the substrate work function if the electronegativity of the adsorbate is higher than that of the metal. If the $\mathrm{MoS}_{2}$ layer is chemisorbed, its electronic structure is perturbed to an extend that a Schottky barrier cannot be defined. However, a second adsorbed $\mathrm{MoS}_{2}$ layer then shows the characteristics of a single unperturbed layer. 


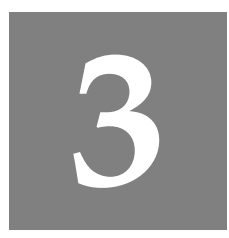

\section{Controlling the Schottky barrier at $\mathrm{MoS}_{2} \mid$ metal contacts by inserting a BN monolayer}

* Making a metal contact to the two-dimensional semiconductor $\mathrm{MoS}_{2}$ without creating a Schottky barrier is a challenge. Using density functional calculations we show that, although the Schottky barrier for electrons obeys the Schottky-Mott rule for high work function $\left(\gtrsim_{4} .7\right.$ $\mathrm{eV}$ ) metals, the Fermi level is pinned at $0.1-0.3 \mathrm{eV}$ below the conduction band edge of $\mathrm{MoS}_{2}$ for low work function metals, due to the metal-MoS $\mathrm{S}_{2}$ interaction. Inserting a boron nitride (BN) monolayer between the metal and the $\mathrm{MoS}_{2}$ disrupts this interaction, and restores the $\mathrm{MoS}_{2}$ electronic structure. Moreover, a BN layer decreases the metal work function of Co and $\mathrm{Ni}$ by $\sim 2 \mathrm{eV}$, and enables a line-up of the Fermi level with the $\mathrm{MoS}_{2}$ conduction band. Surface modification by adsorbing a single BN layer is a practical method to attain vanishing Schottky barrier heights.

\subsection{Introduction.}

Single layers of transition metal dichalcogenides (TMDs) such as molybdenite, $\mathrm{MoS}_{2}$, can be exfoliated through micromechanical cleavage, similar to graphene [68]. In contrast to graphene however, a $\mathrm{MoS}_{2}$ monolayer is a semiconductor with a sizable band gap of $1.8 \mathrm{eV}$ [25], which has triggered a large interest in TMD semiconductor devices $[69,123,124]$. Contacting $\mathrm{MoS}_{2}$ to metal electrodes remains a problem, as it tends to produce unexpectedly high contact barriers and resistances. Early photoemission experiments claimed that the Schottky barriers at $\mathrm{MoS}_{2} \mid$ metal inter-

${ }^{*}$ This chapter has been published as: M. Farmanbar and G. Brocks, Controlling the Schottky barrier at $\mathrm{MoS}_{2} \mid$ metal contacts by inserting a BN monolayer, Phys. Rev. B 91, 161304(R) (2015). 
faces obey the ideal Schottky-Mott rule [107], suggesting the possibility to control the Schottky barrier height (SBH). In particular, the SBH for electrons might be reduced to zero using a metal with a sufficiently low work function. However, the more recent device experiments do not give zero SBHs, neither for metals with high work functions, nor for metals with low work functions [71, 72, 73, 74, 75, 76].

SBHs of metal contacts with conventional semiconductors such as $\mathrm{Si}$ often only weakly depend on the metal species, and the Fermi level is pinned inside the semiconductor band gap [28]. Common models used to explain Fermi level pinning rely upon having a strong (chemical) interaction at the metal-semiconductor interface. This yields a large density of interface states with energies in the semiconductor band gap, which can be suppressed only by inserting an insulating layer between the metal and the semiconductor $[125,126]$. Unlike $\mathrm{Si}, \mathrm{MoS}_{2}$ has no dangling bonds at its surface. Its interaction with metal surfaces should therefore be relatively weak, which makes it rather unlikely that midgap interface states are formed at a high density. Indeed a recent density functional theory (DFT) study claims there is only a weak Fermi level pinning at $\mathrm{MoS}_{2} \mid$ metal interfaces [84]. That still leaves the prospect of a zero SBH using a metal that has a sufficiently low work function.

In this paper we study the Schottky barriers at $\mathrm{MoS}_{2} \mid$ metal interfaces by DFT calculations with the objective of designing a contact with zero SBH. We start from a series of metals covering a wide range of work functions (3.8-5.8 eV). Unlike previous studies we do not so much focus on the chemical interactions with specific metals [82, 83, 127], but on establishing general rules for the SBHs. We show that for clean $\mathrm{MoS}_{2} \mid$ metal interfaces with high work function metals the Fermi level is not pinned in the $\mathrm{MoS}_{2}$ band gap, and the SBH shows Schottky-Mott behavior [28]. This breaks down for low work function metals, and the Fermi level gets pinned just below the $\mathrm{MoS}_{2}$ conduction band, leading to a finite SBH in the range $0.1-0.3 \mathrm{eV}$. The metal- $\mathrm{MoS}_{2}$ interaction at the interface perturbs the electronic structure of $\mathrm{MoS}_{2}$, its conduction bands in particular, creating a density of interface states just below the conduction band that pins the Fermi level.

We "unpin" the Fermi level by inserting a $h$-BN monolayer between the metal surface and $\mathrm{MoS}_{2}$. It breaks the direct metal-MoS interaction and destroys the interface states. Like graphene, $\mathrm{MoS}_{2}$ is physisorbed on the $h$-BN-covered substrate [128, 129], which leaves its electronic structure nearly unperturbed. Moreover, adsorption of $h$-BN on a metal surface commonly decreases the work function considerably. In particular, $h$-BN is lattice matched to $\mathrm{Co}$ and $\mathrm{Ni}(111)$, and turns these high work function metals into low work function substrates. The combined effects of breaking the metal- $\mathrm{MoS}_{2}$ interaction and lowering the metal work function yields zero SBHs for contacts between $h$-BN-covered $\mathrm{Co}$ or $\mathrm{Ni}$ and $\mathrm{MoS}_{2}$.

\subsection{Computational details.}

We use projector augmented waves (PAW) as implemented in the VASP code [44, 45, $46,47]$. The $\mathrm{MoS}_{2} \mid$ metal interface is modeled by a slab of four layers of metal atoms 
with a monolayer $\mathrm{MoS}_{2}$ adsorbed on one side, and a dipole correction to avoid an interaction between repeated images [97]. We optimize all atomic positions, keeping the layer of metal atoms furthest removed from the adsorbant in its bulk geometry, and using energy and force convergence criteria of $10^{-5} \mathrm{eV}$ and $10^{-2} \mathrm{eV} / \AA$, respectively. The surface Brillouin zone is integrated using the Methfessel-Paxton technique with a smearing parameter of $0.05 \mathrm{eV}$ [96], and a $k$-point grid with a spacing of $0.01 \AA^{-1}$. The kinetic energy cutoff for plane waves is set at $400 \mathrm{eV}$. As the properties of $\mathrm{MoS}_{2}$ are sensitive to its lattice parameter [101], we fix the latter to its optimized value and force the metal lattice to be commensurable to the $\mathrm{MoS}_{2}$ lattice, choosing in-plane supercells with a mismatch between the $\mathrm{MoS}_{2}$ and the metal lattices of less than $1 \%$. For instance, putting a $\sqrt{3} \times \sqrt{3} R 30^{\circ} \mathrm{MoS}_{2}$ lattice on top of a $2 \times 2 \mathrm{Au}(111)$ lattice [84, 83], requires decreasing the metal lattice parameter by $4.2 \%$ (PBE), whereas $\sqrt{13} \times \sqrt{13} R 13.9^{\circ} \mathrm{MoS}_{2}$ on $4 \times 4 \mathrm{Au}(111)$, see Fig. 3.1, involves decreasing it by $0.15 \%$. The difference in the interface potential step between these two structures is $\sim 0.1 \mathrm{eV}$.

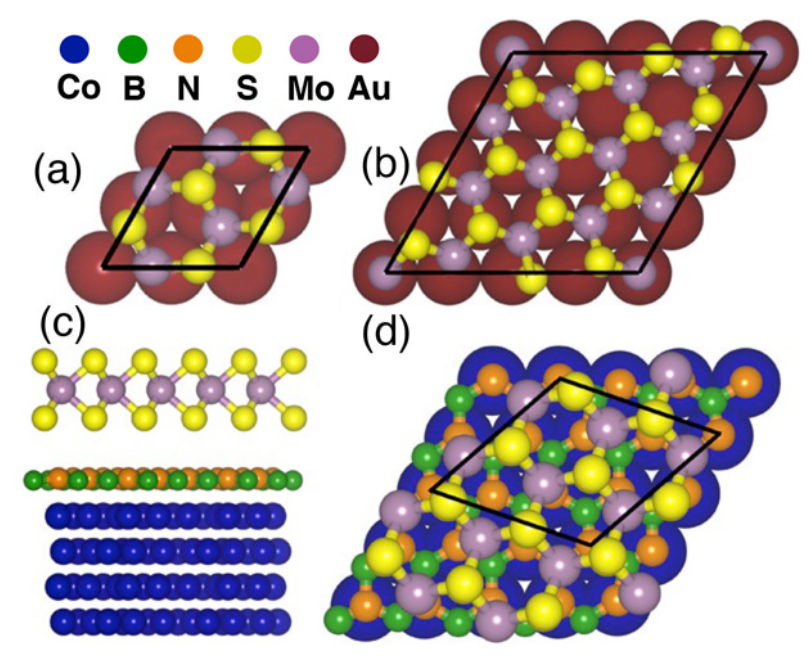

Figure 3.1: (Color online) (a) $\sqrt{3} \times \sqrt{3} R 30^{\circ} \mathrm{MoS}_{2}$ on top of $2 \times 2 \mathrm{Au}(111)$. The black rhombus indicates the surface supercell. (b) $\sqrt{13} \times \sqrt{13} R 13.9^{\circ} \mathrm{MoS}_{2}$ on top of $4 \times 4$ $\mathrm{Au}(111)$. (c,d) Top and side view of the $\mathrm{MoS}_{2}|h-\mathrm{BN}| \mathrm{Co}(111)$ structure.

The metal-adsorbant binding distance is important for obtaining an accurate interface potential profile, and in some cases this distance depends sensitively on the DFT functional [40]. The PBE generalized gradient approximation [59] and the optB88-vdW-DF van der Waals density functional (vdW-DF) [63, 64] give $\mathrm{MoS}_{2} \mid \mathrm{Ag}$, $\mathrm{Au}, \mathrm{Pd}$ and $\mathrm{Pt}(111)$ binding distances within $0.1 \AA$ of one another and interface potential steps within $0.05 \mathrm{eV}$. The local density approximation (LDA) [55] yields on average $\sim 0.2 \AA$ shorter binding distances and $\sim 0.15 \mathrm{eV}$ larger potential steps. We use PBE in the following to avoid the risk of overbinding commonly found with LDA. Whereas the interface potential step does not depend too critically on the DFT 
functional, the work function of a clean metal surface $W_{\mathrm{M}}$ can be more sensitive $[78,84]$, which is then reflected in the calculated SBHs, see Eq. (3.1).

\section{3 $\mathrm{MoS}_{2} \mid$ metal interfaces.}

The SBH for electrons can be written as

$$
\Phi_{\mathrm{n}}=W_{\mathrm{M}}-\chi-\Delta V
$$

with $W_{\mathrm{M}}$ the work function of the clean metal surface, $\chi$ the electron affinity of $\mathrm{MoS}_{2}$, and $\Delta V$ the potential step formed at the $\mathrm{MoS}_{2} \mid$ metal interface, see Fig. 3.2. The potential step can be calculated without resorting to the details of the potential profile across the interface, or its electronic structure, because $\Delta V=W_{\mathrm{M}}-W_{\mathrm{ads} \mid \mathrm{M}}$, where $W_{\text {ads } \mid \mathrm{M}}$ is the work function of the metal surface covered with $\mathrm{MoS}_{2}$ [97]. The results are shown in Fig. 3.2(a). The SBH is then obtained from Eq. (3.1), using the calculated $\chi=4.30 \mathrm{eV}$, cf. Fig. 3.2(b). Two regimes can be distinguished in Fig. 3.2(a). Metals with $W_{\mathrm{M}} \gtrsim 4.7 \mathrm{eV}$ yield a similar $\Delta V$, whereas $\Delta V$ strongly depends on $W_{\mathrm{M}}$ for $W_{\mathrm{M}} \lesssim 4.7 \mathrm{eV}$. The cross-over between the two regimes occurs for $\mathrm{Cu}$, where $W_{\mathrm{Cu}} \approx \chi+\Delta V$ and the $\mathrm{SBH}$ is minimal, see Fig. 3.2(b). The results for the high work function regime are consistent with the common observation that physisorption results in a net decrease of the work function, i.e., a positive $\Delta V$. This has been explained in terms of the Pauli exchange repulsion between the electrons of the metal and those of the overlayer yielding a net pushback of electrons into the metal [40]. The effect is fairly moderate for $\mathrm{MoS}_{2}$, resulting in $\Delta V \approx 0.35 \mathrm{eV}$ for the high work function metals, and a size that does not depend critically on the details of the metal or the interface structure.

With a constant $\Delta V$ the SBH simply follows the work function, i.e., the slope $S=$ $d \Phi_{\mathrm{n}} / d W_{\mathrm{M}} \approx 1$, which is the Schottky-Mott rule. This rule is typically found in the absence of any interface states with energies in the semiconductor band gap, which is consistent with the $\mathrm{MoS}_{2} \mid$ metal interaction being weak. If ideal Schottky-Mott behavior would persist for the low work function metals, the SBH would vanish for $W_{\mathrm{M}}<W_{\mathrm{Cu}}$. Clearly this is not the case in Fig. 3.2(b). The SBH has a minimum at $\mathrm{Cu}$, but it increases again for the low work function metals.

We analyze this behavior for $\mathrm{Mg}$, being the metal with the lowest work function in this study. The band structure of the $(1 \times 1) \mathrm{MoS}_{2} \mid \mathrm{Mg}(0001)$ interface is shown in Fig. 3.3(a). At the optimized equilibrium distance $d=2.2 \AA$, the $\mathrm{MoS}_{2}$ bands are significantly perturbed by the interaction with the substrate. This perturbation can be visualized by comparing the density of states (DoS) of $\mathrm{MoS}_{2} \mid \mathrm{Mg}$ to that of free-standing $\mathrm{MoS}_{2}$, see Fig. 3.3(e). The $\mathrm{MoS}_{2} \mid \mathrm{Mg}$ interaction leads to interface states in the $\mathrm{MoS}_{2}$ band gap that are energetically close to the bottom of the $\mathrm{MoS}_{2}$ conduction band. This is seen most clearly in the difference DoS, i.e. $\Delta \mathrm{DoS}=\operatorname{DoS}_{\mathrm{ads} \mid \mathrm{M}}-\operatorname{DoS}_{\mathrm{ads}}-\operatorname{DoS}_{\mathrm{M}}$, represented by the green curve in Fig. 3.3(e), where $\operatorname{DoS}_{\mathrm{ads} \mid \mathrm{M}}, \mathrm{DoS}_{\mathrm{ads}}, \mathrm{DoS}_{\mathrm{M}}$ are the DoSs of the interface, the free-standing adsor- 


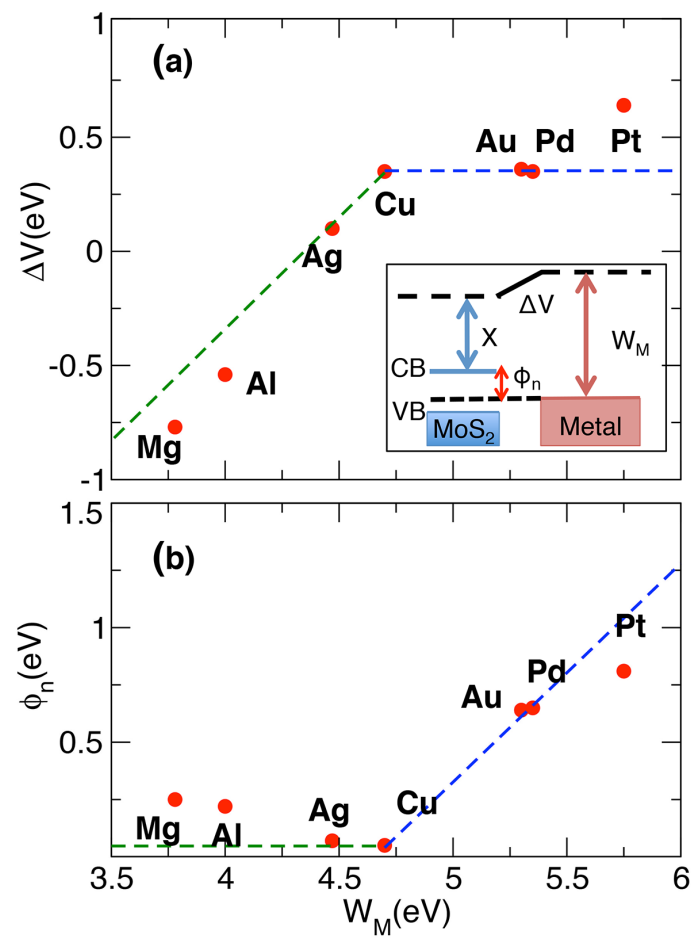

Figure 3.2: (Color online) (a) The potential step $\Delta V$ at the $\mathrm{MoS}_{2} \mid$ metal interface versus $W_{\mathrm{M}}$, the work function of the clean metal surface. (b) The Schottky barrier height (SBH) for electrons $\Phi_{n}$. The blue and green dashed lines in (a) and (b) indicate the Schottky-Mott rule and Fermi level pinning, respectively. Inset: schematic energy diagram of the interface, with $\chi$ the electron affinity of $\mathrm{MoS}_{2}$.

bate, and the clean metal substrate, respectively.

The $\Delta \mathrm{DoS}$ is negligible for energies inside the $\mathrm{MoS}_{2}$ band gap, except for a region $\lesssim 0.4 \mathrm{eV}$ below the conduction band edge (shaded orange in Fig. 3.3(e)). The negative sign of the calculated potential step at the $\mathrm{MoS}_{2} \mid \mathrm{Mg}$ interface, $\Delta V=-0.77$ $\mathrm{eV}$, shows that electronic charge is transferred from the $\mathrm{Mg}$ substrate to the $\mathrm{MoS}_{2}$ overlayer. These electrons populate the interface states, thereby pinning the Fermi level in the band gap. For high work function metals the Fermi level is well within the $\mathrm{MoS}_{2}$ gap, where the density of interface states is negligible. The corresponding $\mathrm{SBH}$ then obey the Schottky-Mott rule. It is only for low work function metals, when the Fermi level approaches the bottom of the $\mathrm{MoS}_{2}$ conduction band, that interface states become noticeable and pin the Fermi level.

One can destroy the interface states by breaking the $\mathrm{MoS}_{2} \mid \mathrm{Mg}$ interaction. This is demonstrated by Fig. 3.3(b), which gives the band structure of $\mathrm{MoS}_{2} \mid \mathrm{Mg}(0001)$ with the adsorbant placed at an artificially large distance $d=5 \AA$ from the metal surface. The $\mathrm{MoS}_{2}$ bands are unperturbed, as there is no chemical interaction with 

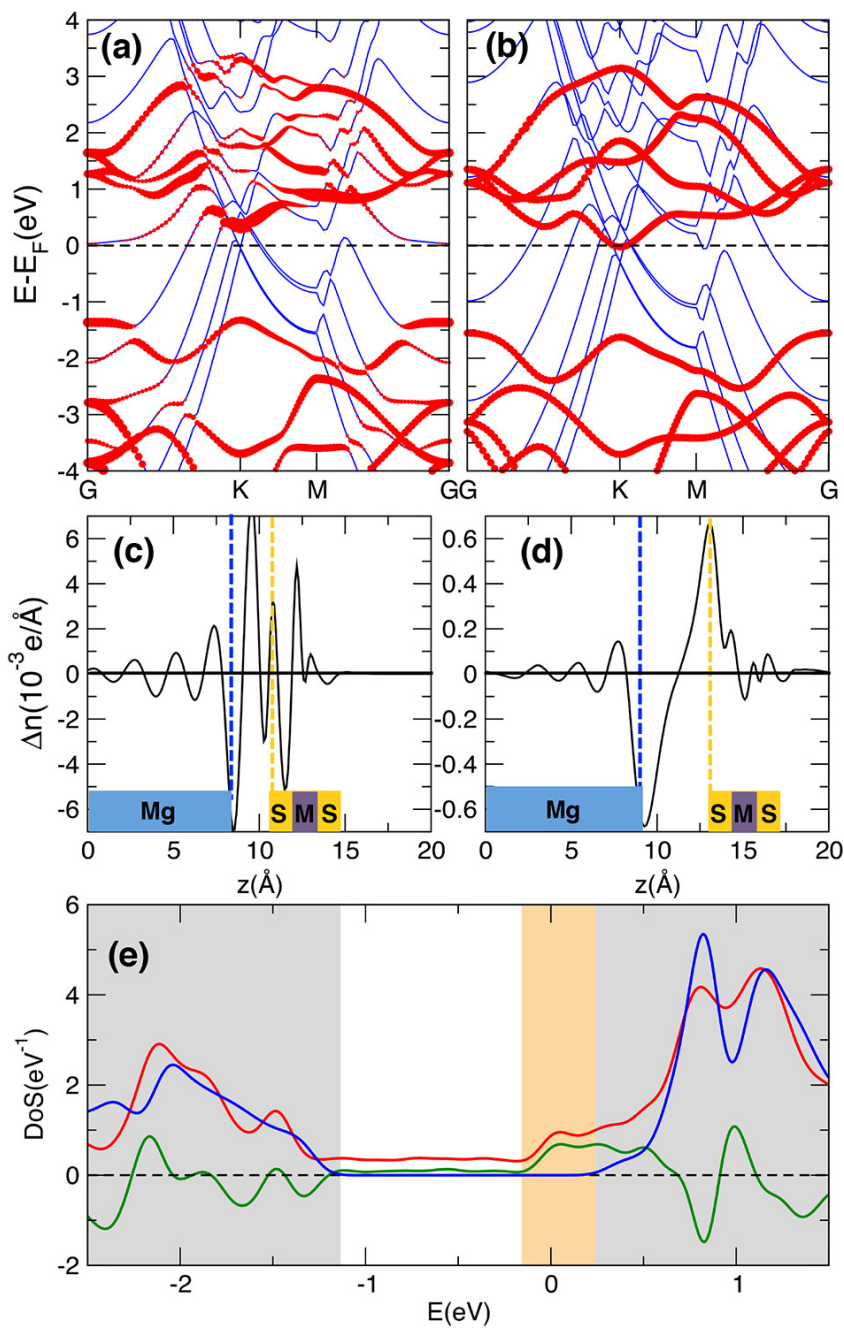

Figure 3.3: (Color online) (a) Band structures of the $\mathrm{MoS}_{2} \mid \mathrm{Mg}(0001)$ interfaces at the equilibrium distance $d_{\mathrm{eq}}=2.2 \AA$ and (b) at $d=5 \AA$. The red color measures a projection of the wave function on the $\mathrm{MoS}_{2}$ orbitals. The Fermi level is set at zero energy. (c,d) The electron difference density $\Delta n(z)$, corresponding to the interface distances of $(a, b)$, respectively. (e) The total density of states (red) corresponding to (a) $\mathrm{DoS}_{\mathrm{ads} \mid \mathrm{Mg}}$, (blue) of free-standing $\mathrm{MoS}_{2} \mathrm{DoS}_{\mathrm{ads}}$, and (green) the difference $\Delta \mathrm{DoS}=\operatorname{DoS}_{\mathrm{ads} \mid \mathrm{Mg}}-\mathrm{DoS}_{\mathrm{ads}}-\mathrm{DoS}_{\mathrm{Mg}}$. The orange shading indicates the position of the gap states, created by the interaction at the interface. 
the substrate at this distance. The Fermi level is in the $\mathrm{MoS}_{2}$ conduction band, which is not surprising as the work function of $\mathrm{Mg}(0001), W_{\mathrm{Mg}}=3.8 \mathrm{eV}$ is much smaller than the $\mathrm{MoS}_{2}$ electron affinity $\chi=4.3 \mathrm{eV}$. The calculated interface potential step, $\Delta V_{\mathrm{ni}}=-0.5 \mathrm{eV}$, indeed corresponds to $W_{\mathrm{Mg}}-\chi$, as it should for electron transfer from $\mathrm{Mg}$ to $\mathrm{MoS}_{2}$ to equilibrate the Fermi level. It results in a zero SBH, according to Eq. (3.1).

This interpretation is confirmed by the electron density difference $\Delta n(z)=n_{\text {ads } \mid \mathrm{M}}(z)-n_{\text {ads }}(z)-n_{\mathrm{M}}(z)$, where $n_{\text {ads } \mid \mathrm{M}}(z), n_{\text {ads }}(z), n_{\mathrm{M}}(z)$ are the planeaveraged electron densities of the interface, the free-standing adsorbate, and the clean metal substrate, respectively. At an $\mathrm{MoS}_{2} \mid \mathrm{Mg}$ distance $d=5 \AA, \Delta n(z)$ shows an accumulation of electrons at the position of $\mathrm{MoS}_{2}$, and an electron depletion at the $\mathrm{Mg}$ surface, see Fig. 3.3(d), consistent with an electron transfer from $\mathrm{Mg}$ to $\mathrm{MoS}_{2}$, which creates an interface dipole and potential step to equilibrate the Fermi level. At the equilibrium $\mathrm{MoS}_{2} \mid \mathrm{Mg}$ distance, $\Delta n(z)$ shows a much more complicated pattern, see Fig. 3.3(c), which is consistent with an interface interaction that alters the electronic structure.

\section{4 $\operatorname{MoS}_{2} \mid \boldsymbol{h}$-BN $\mid$ metal interfaces.}

A Schottky barrier at a $\mathrm{MoS}_{2} \mid$ metal contact is unavoidable, as the interface interaction leads to states that pin the Fermi level below the conduction band. Breaking this interaction by introducing a vacuum spacing at the interface, is not a practical way to make a metal-semiconductor contact. However, inserting an inert layer between the metal and $\mathrm{MoS}_{2}$ can be. This layer has to be sufficiently thin as not to form a high barrier for electron transport. A monolayer of $h$-BN is ideally suited. A single layer of $h$-BN can be deposited or grown on a range of metal substrates, and it is stable under ambient conditions. Substrates consisting of a transition metal (111) surface covered by a $h$-BN monolayer, are widely available. Sandwiching a monolayer of $h$-BN between two metal electrodes gives metallic conduction [130], which indicates that the layer is transparent to electrons.

We build $\mathrm{MoS}_{2} \mid h$-BN|metal structures by putting a $(2 \times 2) \mathrm{MoS}_{2}$ cell on top of a $(\sqrt{7} \times \sqrt{7}) h$-BN $\mid$ Co or $\mathrm{Ni}$ cell, see Fig. 3.1. The $h$-BN layer in a $(1 \times 1)$ registry with the underlying metal surface [40,78], which agrees with experiment [131]. As before, we fix the lattice constant of $\mathrm{MoS}_{2}$, and adapt the lattices of $h$-BN and the metal(111) substrates accordingly, which requires a $4 \%$ squeeze of the $h$-BN lattice. A $h$-BN monolayer is chemisorbed on Co and Ni(111) surfaces, but $\mathrm{MoS}_{2}$ and $h$ $\mathrm{BN}$ are bonded by a weak, van der Waals, interaction. Such interactions are not represented in the PBE functional, so we use the optB88-vdW-DF van der Waals density functional here.

Adsorption of $h$-BN has a dramatic effect on the work function; it reduces the work functions of $\mathrm{Co}$ and $\mathrm{Ni}(111)$ by $1.9 \mathrm{eV}$ and $1.8 \mathrm{eV}$, respectively. These reductions result from large interface dipoles that are formed at the $h-\mathrm{BN} \mid$ metal interfaces, where Pauli exchange repulsion between the electrons at the interface gives an im- 


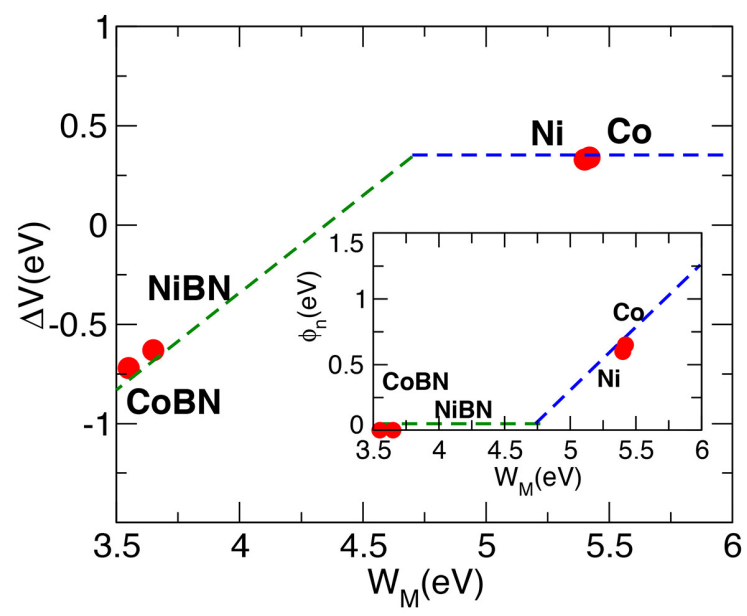

Figure 3.4: (Color online) $\Delta V$ versus $W_{\mathrm{M}}$. Inset: $\Phi_{n}$ versus $W_{\mathrm{M}}$. The blue and green dashed lines indicate the Schottky-Mott rule and Fermi level pinning, as in Fig. 3.2.

portant contribution [40]. Adsorbants in the form of self-assembled monolayers (SAMs) are commonly proposed in order to modify substrate work functions. However, SAMs often suffer from disorder, which diminishes their effect. Adsorption of $h$-BN leads to a well-defined structure that is much less susceptible to disorder, and gives a sizable work function lowering. Moreover $h$-BN presents a surface that not only is chemically relatively inert, but also does not change its structure upon adsorbing further layers.

The implications of work function lowering by $h$-BN adsorption are clearly demonstrated in Fig. 3.4. Direct adsorption of $\mathrm{MoS}_{2}$ on Co and Ni(111) gives a behavior that is typical for high work function metals. The potential step at the $\mathrm{MoS}_{2} \mid$ metal interface is $\Delta V \approx 0.35 \mathrm{eV}$, and the SBHs follow the Schottky-Mott rule. In contrast, adsorption of $\mathrm{MoS}_{2}$ on $h$-BN|Co and $\mathrm{Ni}(111)$ substrates gives a negative $\Delta V$, and it gives a zero SBH. Inserting a $h$-BN layer has not only effectively decreased the substrate work function, but it has also weakened the $\mathrm{MoS}_{2} \mid$ substrate interaction that yielded Fermi level pinning and nonzero SBHs for clean low work function metal substrates. The difference between adsorbing $\mathrm{MoS}_{2}$ directly onto a clean Co surface and onto a $h$-BN covered Co surface is observed in the corresponding electronic structures shown in Fig. 3.5. Direct adsorption onto $\mathrm{Co}(111)$ perturbs the $\mathrm{MoS}_{2}$ bands considerably, due to the interaction at the interface, as shown in Fig. 3.5(a). In contrast, adsorbing $\mathrm{MoS}_{2}$ onto a $h$-BN|Co(111) substrate hardly perturbs the $\mathrm{MoS}_{2}$ bands at all, as demonstrated by Fig. 3.5(b), which is a clear indication that the interaction between $h$-BN and $\mathrm{MoS}_{2}$ is weak.

Adsorbing $\mathrm{MoS}_{2}$ directly onto Co leads to the Fermi level being well within the $\mathrm{MoS}_{2}$ band gap, which is not surprising as Co is a high work function metal. In contrast, adsorbing $\mathrm{MoS}_{2}$ onto a $h-\mathrm{BN} \mid \mathrm{Co}$ substrate yields a Fermi level that is pinned at the bottom of the $\mathrm{MoS}_{2}$ conduction band. The electron density differences $\Delta n(z)$ 

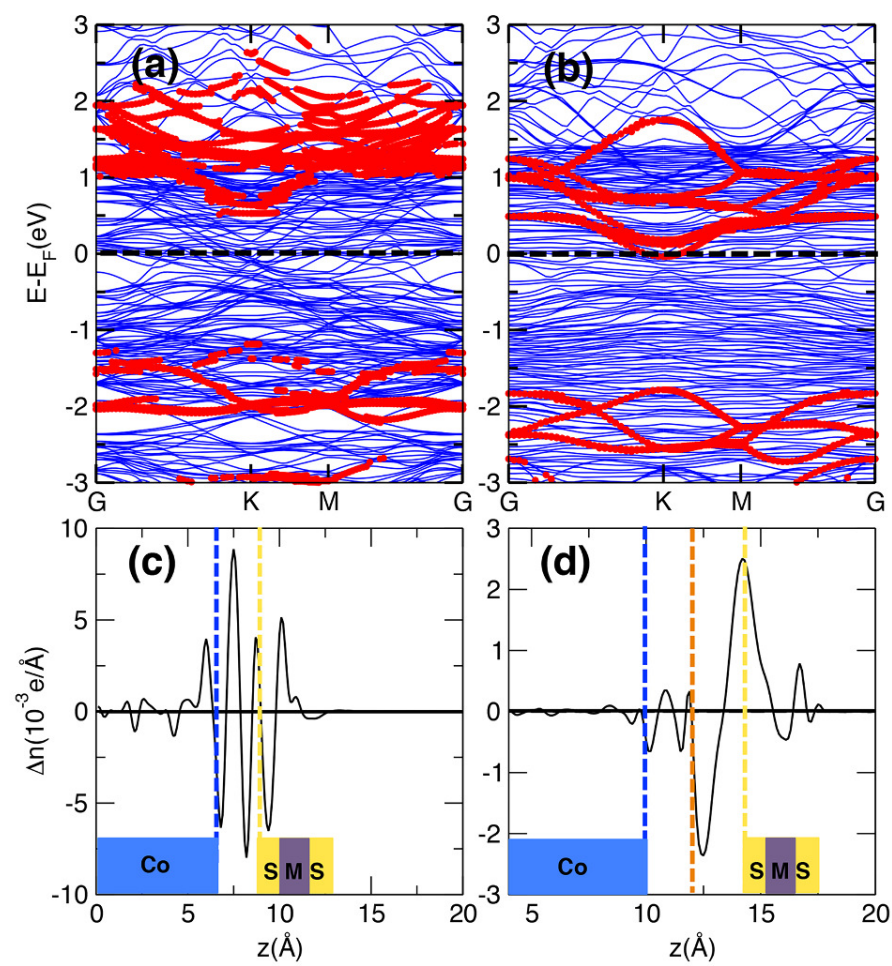

Figure 3.5: (Color online) (a) Band structures of the $\mathrm{MoS}_{2} \mid \mathrm{Co}(111)$ and (b) the $\mathrm{MoS}_{2}|h-\mathrm{BN}| \mathrm{Co}(111)$ interfaces. The red color measures a projection of the wave function on the $\mathrm{MoS}_{2}$ orbitals. The Fermi level is set at zero energy. (c,d) The corresponding electron difference density $\Delta n(z)$. The orange line indicates the position of the $h$-BN layer.

shown in Fig. 3.5(c) and 3.5(d) substantiate this picture. Figure 3.5(c) shows the complicated pattern that is typically associated with a direct $\mathrm{MoS}_{2} \mid$ metal interaction, compare to Fig. 3.3(c). $\Delta n(z)$ for $\mathrm{MoS}_{2}$ on a $h$-BN|Co shows an accumulation of electrons at the position of $\mathrm{MoS}_{2}$, and an electron depletion at the position of the $h$ $\mathrm{BN}$, which reflects an electron transfer from the substrate to $\mathrm{MoS}_{2}$ to equilibrate the Fermi level, compare Figs 3.5(d) and 3.3(d).

\subsection{Conclusions.}

We have shown that contacting $\mathrm{MoS}_{2}$ with low work function metals leads to Fermi level pinning at $0.1-0.3 \mathrm{eV}$ below the conduction band edge. This behavior results from the interaction at the interface between the metal and the $\mathrm{MoS}_{2}$, creating a considerable density of interface states just below the $\mathrm{MoS}_{2}$ conduction band. Inserting a boron nitride (BN) monolayer between the metal and the $\mathrm{MoS}_{2}$ destroys these interface states, and recovers the unperturbed $\mathrm{MoS}_{2}$ band structure. In addi- 
tion, absorbing $h$ - $\mathrm{BN}$ on $\mathrm{Co}(111)$ or $\mathrm{Ni}(111)$ decreases the metal work function by close to $2 \mathrm{eV}$. We predict that contacting $\mathrm{MoS}_{2}$ with $h-\mathrm{BN} \mid \mathrm{Co}$ or $\mathrm{Ni}(111)$ does not give a Schottky barrier. 


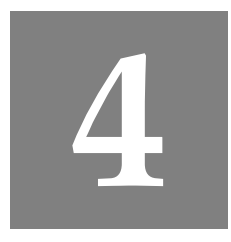

\section{Ohmic contacts to 2D semiconductors through van der Waals bonding}

* High contact resistances have blocked the progress of devices based on $M_{2}(M=M o, W ; X$ $=S, S e, T e) 2 D$ semiconductors. Interface states formed at $M_{2} /$ metal contacts pin the Fermi level, leading to sizable Schottky barriers for p-type contacts in particular. We show that (i) one can remove the interface states by covering the metal by a 2D layer, which is van der Waals-bonded to the $\mathrm{MX}_{2}$ layer, and (ii) one can choose the buffer layer such, that it yields a p-type contact with a zero Schottky barrier height. We identify possible buffer layers such as graphene, a monolayer of $h-B N$, or an oxide layer with a high electron affinity, such as $\mathrm{MoO}_{3}$. The most elegant solution is a metallic $\mathrm{M}^{\prime} \mathrm{X}_{2}^{\prime}$ layer with a high work function. A $\mathrm{NbS}_{2}$ monolayer adsorbed on a metal yields a high work function contact, irrespective of the metal, which gives a barrierless contact to all $\mathrm{MX}_{2}$ layers.

\subsection{Introduction}

Layered transition metal dichalcogenides $\mathrm{MX}_{2}, \mathrm{M}=\mathrm{Mo}, \mathrm{W}, \mathrm{X}=\mathrm{S}, \mathrm{Se}, \mathrm{Te}$, are widely explored because of their unique properties and their potential for applications in electronic devices [21, 132]. $\mathrm{MX}_{2}$ monolayers are direct band gap semiconductors with band gaps in the range of $1-2 \mathrm{eV}$, which have appealing electronic and optoelectronic properties $[69,133] . \mathrm{MX}_{2}$ layers can be obtained via micro-mechanical cleaving [68], by chemical vapor deposition (CVD) [134, 135], or even by spin coating precursor molecules [136]. Important for applications in devices is the ability to have both electron (n-type) and hole (p-type) transport in these 2D materials. Charge carrier transport in $\mathrm{MX}_{2}$ field-effect transistors (FETs) is usually dominated by elec-

*This chapter has been published as: M. Farmanbar and Geert Brocks, Ohmic contacts to 2D semiconductors through van der Waals bonding,Adv. Electron. Mater., 1500405 (2016). 
trons; p-type transport has only been demonstrated in $\mathrm{WSe}_{2}[137,138]$.

A major challenge for $\mathrm{p}$-type transport is that $\mathrm{MX}_{2}$ forms a large Schottky barrier (SB) for holes with metals commonly used for making electrical contacts. A standard way to reduce a metal/semiconductor contact resistance is to heavily dope the semiconductor in the contact region, which effectively decreases the SB width. Local doping of a 2D semiconductor is however very challenging; so far most techniques used for doping 2D materials, such as substitutional doping [139], adsorbed molecules [137, 140, 93], or electrolytes [141, 142], have a limited spatial resolution. Alternatively one tries to decrease the SB height, essentially by covering the metal by a thin layer to increase its work function. Oxides have shown their potential for $\mathrm{p}$ type contacts in organic photovoltaics and light-emitting diodes [143, 144], and have also been tested in $\mathrm{MoS}_{2}$ FETs $[145,146]$. Oxides have also been applied succesfully to reduce the SB height for n-type contacts to $\mathrm{MoS}_{2}$ [82].

Common metals generally give n-type contacts with substantial SB heights, leading to high contact resistances. Although $\mathrm{MX}_{2}$ monolayers are free of dangling bonds, nevertheless they interact with low work-function metals to form a density of interface states with energies inside the $\mathrm{MX}_{2}$ band gap, which is sufficiently large to pin the Fermi level and cause a sizable SB for electrons [77, 83, 84]. We show that high work-function metals yield high SBs for holes by a similar mechanism.

We suggest a practical way to solve the p-type contact problem and tune the SB height by inserting a monolayer of a $2 \mathrm{D}$ material between the metal substrate and the $\mathrm{MX}_{2}$ semiconductor, see figure 4.1. The buffer layer suppresses the metal/ $\mathrm{MX}_{2}$ interface states. 2D materials have certain unique properties not found in buffer layers of 3D materials [68]. As the interlayer bonding is van der Waals, neither the structure of the $2 \mathrm{D}$ buffer layer, nor that of the $\mathrm{MX}_{2}$ semiconductor, is perturbed significantly by stacking them. The $2 \mathrm{D}$ buffer layer need not be lattice matched to the metal or to the $\mathrm{MX}_{2}$ layer, and the structure of the multilayer will in general be incommensurate. Van der Waals interface bonding also promises the absence of interface states. Covering the metal by an adsobant layer such as graphene, a monolayer of hexagonal boron nitride $(h-\mathrm{BN})$ or $T-\mathrm{MoS}_{2}$, has proved to be beneficial for making n-type contacts [77,147]. We show that a 2D buffer layer can be selected to obtain a zero SB height for holes.

A $h$-BN monolayer is a buffer layer that can be used for making n-type contacts, because adsorption of $h$-BN on a metal decreases its work function by up to $1-2 \mathrm{eV}$ [78]. For instance, $\mathrm{Co} / h-\mathrm{BN}$ and $\mathrm{Ni} / h-\mathrm{BN}$ are predicted to form zero SB height $\mathrm{n}$ type contacts to $\mathrm{MX}_{2}$ semiconductors, see figure 4.2 [77]. A decrease of the work function is unfavorable for making $\mathrm{p}$-type contacts. Metal $/ h$-BN gives a p-type contact to $\mathrm{MX}_{2}$ only if the metal work function is sufficiently high, and the $\mathrm{MX}_{2}$ ionization potential is sufficiently low. We find a zero SB height for $\mathrm{Pt} / h$-BN, and $\mathrm{Au} / h$-BN contacts to $\mathrm{MoTe}_{2}$ and a low SB for $\mathrm{Pt} / h$-BN/WSe 2 , see figure 4.2. Alternatively, one can use a graphene buffer layer $[149,150]$, whose behavior is qualitatively similar to that of a $h$-BN monolayer.

To make more universely applicable p-type contacts, one needs a buffer layer that 


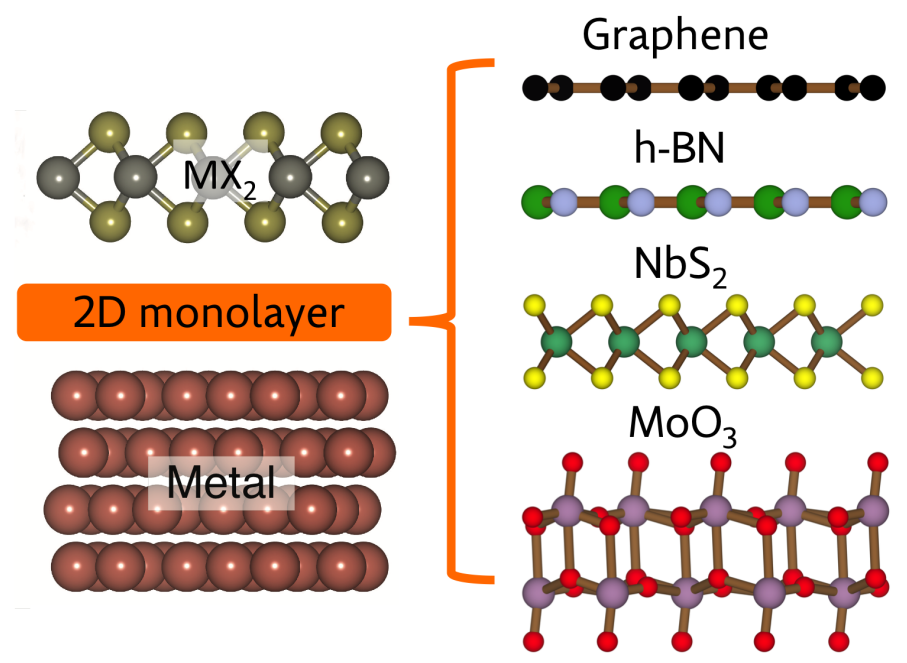

Figure 4.1: Side view of the metal/buffer $/ \mathrm{MX}_{2}$ structure, $\mathrm{M}=\mathrm{Mo}, \mathrm{W}, \mathrm{X}=\mathrm{S}, \mathrm{Se}, \mathrm{Te}$ with possible buffer layers graphene, $h-\mathrm{BN}, \mathrm{NbS}_{2}$, and $\mathrm{MoO}_{3}$. Visualization by VESTA [148].

effectively increases the metal work function. Oxides such as $\mathrm{MoO}_{3}$ are an option. The $\mathrm{MoO}_{3}$ structure consists of bilayers, making it conceivable to cover a metal with a single $\mathrm{MoO}_{3}$ bilayer, see figure 4.1. We find that the electron affinity of $\mathrm{MoO}_{3}$ is sufficiently high to make carrier transport through its conduction band possible, so that a bilayer of $\mathrm{MoO}_{3}$ does not present a tunnel barrier.

A very interesting option for creating p-type contacts is to use metallic $\mathrm{M}^{\prime} \mathrm{X}_{2}^{\prime}$ buffer layers, such as $\mathrm{NbS}_{2}$ or $\mathrm{TaS}_{2}$ [151]. Their structure is similar to that of semiconducting $\mathrm{MX}_{2}$, they are chemically stable, and have work functions close to $6 \mathrm{eV}$. We show that a monolayer $\mathrm{NbS}_{2}$ adsorbed on a metal gives a SB with zero height for contacts to all $\mathrm{MX}_{2}$. The initital work function of the metal substrate is irrelevant; $\mathrm{Au} / \mathrm{NbS}_{2}$ and $\mathrm{Al} / \mathrm{NbS}_{2}$ essentially give the same contact.

\subsection{Results and Discussion}

\subsubsection{Van der Waals bonded contacts}

The obvious way to make a p-type contact to a semiconductor is to use a metal with a high work function. The calculated work function of $\mathrm{Pt}$ is $5.91 \mathrm{eV}$, suggesting that this metal should give a zero $\mathrm{SB}$ to all $\mathrm{MX}_{2}$, except $\mathrm{MoS}_{2}$. In practice this is not true, as $\mathrm{MX}_{2}$ interacts with $\mathrm{Pt}$ to give states at the interface whose energy is within the band gap of $\mathrm{MX}_{2}$. Initially it was thought that $\mathrm{MX}_{2}$ could escape the formation of interface gap states (IGS), as, unlike conventional semiconductors such as $\mathrm{Si}, \mathrm{MX}_{2}$ has no dangling surface bonds that interact strongly with the metal surface [107]. 
n-type contact $\mathrm{MoS}_{2} \quad \mathrm{MoSe}_{2} \quad \mathrm{MoTe}_{2} \mathrm{WS}_{2} \quad \mathrm{WSe}_{2} \quad \mathrm{WTe}_{2} \quad$ p-type contact

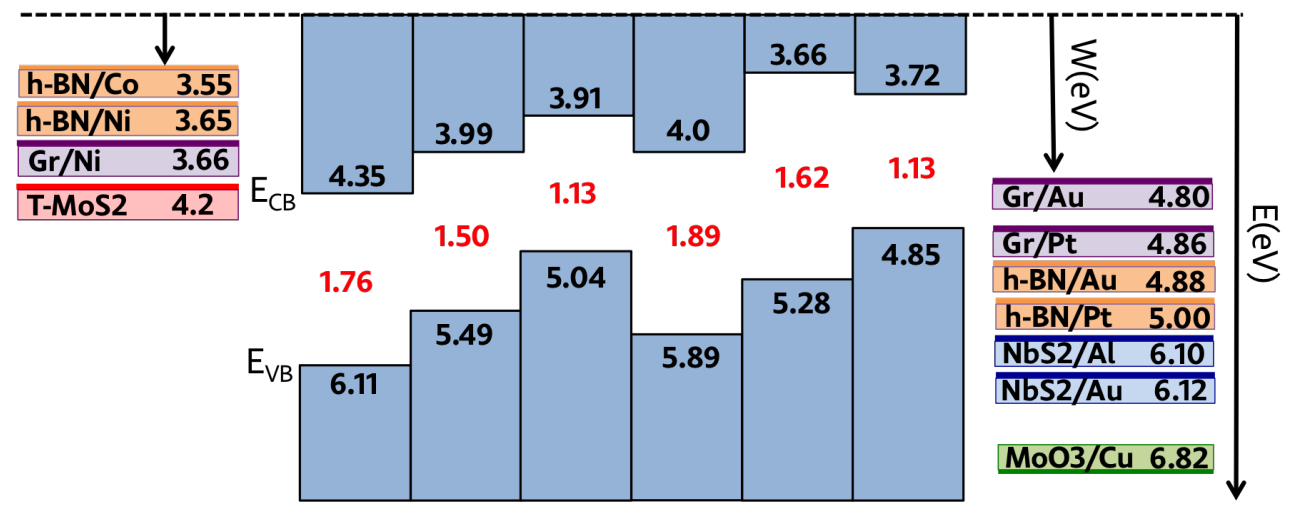

Figure 4.2: Middle: calculated valence band maxima $E_{\mathrm{VB}}$ and conduction band minima $E_{\mathrm{CB}}$ of $H-\mathrm{MX}_{2}$ monolayers (band gaps are given in red), see section 4.4. Left: work functions of n-type metal/buffer contacts [147, 86, 88, 78, 77]. Right: calculated work functions of p-type metal/buffer contacts.

Nevertheless, even a relatively weak interaction yields IGS that pin the Fermi level in the gap, which results in an appreciable SB [77].

As an example, figure 4.3(a) gives the band structure of the $\mathrm{Pt}(111) / \mathrm{MoTe}_{2}$ interface. The direct interaction between $\mathrm{MoTe}_{2}$ and the Pt surface perturbs the band structure of $\mathrm{MoTe}_{2}$ significantly, the valence bands in particular. The perturbation is accompanied by the formation of IGS inside the $\mathrm{MoTe}_{2}$ band gap, figure 4.3(b), which pin the Fermi level. The SB height is defined as

$$
\Phi_{\mathrm{p}}=E_{\mathrm{VB}}-E_{\mathrm{F}},
$$

with $E_{\mathrm{F}}$ the Fermi energy, and $E_{\mathrm{VB}}$ the energy of the top of the valence band (measured as distances, i.e., positive numbers, from the vacuum level). The SB to an electronically perturbed overlayer is of course not extremely well-defined. One can estimate the SB by aligning the core levels of the adsorbed $\mathrm{MoTe}_{2}$ layer to those of a free-standing $\mathrm{MoTe}_{2}$ layer, which gives $\Phi_{\mathrm{p}}=0.49 \mathrm{eV}$. With $E_{\mathrm{VB}}=5.04 \mathrm{eV}$ (figure 4.1) this gives $W_{\mathrm{M}_{\mid \mathrm{WTe}}}=4.55 \mathrm{eV}$ as the work function of of Pt covered by a $\mathrm{MoTe}_{2}$ monolayer. As the calculated work function of clean $\mathrm{Pt}(111)$ is $5.91 \mathrm{eV}$, it implies that adsorbing $\mathrm{MoTe}_{2}$ on Pt creates a large potential step at the interface of 1.36 $\mathrm{eV}$.

The changes in the electronic structure of the adsorbed $\mathrm{MoTe}_{2}$ layer are visualized in figure 4.3(b). Starting from the total density of states (DoS) of the $\mathrm{Pt}(111) / \mathrm{MoTe}_{2}$ system, and subtracting the DoS of the clean $\mathrm{Pt}(111)$ slab, one can compare the result to the $\mathrm{DoS}$ of a free-standing $\mathrm{MoTe}_{2}$ layer. The comparison shows considerable differences in the $\mathrm{MoTe}_{2}$ band gap region, which are direct evidence for the formation of IGS. 
The pattern of these IGS depends on the particular metal/ $\mathrm{MX}_{2}$ combination. We have however not found an elemental high work function metal that does not give IGS. For all high work function metal/ $\mathrm{MX}_{2}$ contacts we have studied, IGS are formed that pin the Fermi level and yield a sizable SB. The same problem has emerged previously for low work function metals and n-type SBs. Introducing a buffer layer can break the interaction between $\mathrm{MX}_{2}$ and the metal and eliminate the IGS. This layer must be sufficiently thin, such that it does not form a large barrier for the charge carriers. In addition, the interaction between $\mathrm{MX}_{2}$ and the buffer layer must not create new IGS.

A single atomic layer of graphene or $h$-BN obeys these criteria. Such a layer presents only a thin barrier that essentially allows for metallic transport through the layer [130]. Inserting graphene or a $h$-BN monolayer between a metal surface and $\mathrm{MX}_{2}$ disrupts the metal-MX $\mathrm{X}_{2}$ chemical interaction, and destroys any metal-induced IGS. Graphene or $h$-BN bind to $\mathrm{MX}_{2}$ via van der Waals forces. One does not expect such an interaction to create new IGS. This is illustrated for $\mathrm{Pt}(111) / h-\mathrm{BN} / \mathrm{MoTe}_{2}$ in figures 4.3(c) and (d). Inserting a monolayer of $h$-BN restores the electronic structure of $\mathrm{MoTe}_{2}$, where the projected bands are essentially those of free-standing $\mathrm{MoTe}_{2}$. The DoS of $\mathrm{Pt}(111) / h-\mathrm{BN} / \mathrm{MoTe}_{2}$ minus that of $\mathrm{Pt}(111)$ is essentially identical to the DoS of a free-standing $\mathrm{MoTe}_{2}$ layer, in particular in the gap region. In other words, it shows no sign of IGS generated by any $h$-BN/MoTe $e_{2}$ interaction.

The concept also works if one uses graphene to cover the metal. The interaction between metal/graphene or metal/ $h$-BN and $\mathrm{MX}_{2}$ is van der Waals, so the electronic structure of any $\mathrm{MX}_{2}$ is preserved, and IGS are absent. A serious drawback however is that adsorption of graphene or $h$-BN generally decreases the metal work function considerably, e.g., by $0.6-1.1 \mathrm{eV}$ for $\mathrm{Pt}$ and $\mathrm{Au}$, see table 4.1. The reduction originates from a dipole layer that is formed at the metal/graphene or metal/h-BN interface, where Pauli exchange repulsion gives an dominant contribution [40].

The reduction is partly canceled by a potential step $\Delta V$ formed at the graphene $/ \mathrm{MX}_{2}$ or $h-\mathrm{BN} / \mathrm{MX}_{2}$ interface, see figure 4.4. Although the weak interaction between $h$ $\mathrm{BN}$ and $\mathrm{MX}_{2}$ does not give IGS, it does lead to an interface potential step, which originates from the Pauli repulsion between the electrons from the $h$-BN layer and those originating from the $\mathrm{MX}_{2}$ layer [40]. As the chalcogenide atoms form the outer atomic layers in $\mathrm{MX}_{2}$, it is then not surprising that $\Delta V$ depends on the chalcogenide species, but not on the metal species of the central atomic layer, see table 4.1. For graphene $/ \mathrm{MX}_{2}$ and $h$ - $\mathrm{BN} / \mathrm{MX}_{2}$ interfaces $\Delta V$ is positive toward $\mathrm{MX}_{2}$ i.e., it decrease the SB. The steps are actually quite moderate, i.e. in the range $0.2-0.3 \mathrm{eV}$, see table 4.1, so they do not cancel out the work function reductions discussed in the previous paragraph.

One obtains a zero SB height only with graphene- or $h$-BN-covered metals with a high work function, such as $\mathrm{Pt}$ and $\mathrm{Au}$, in contact with $\mathrm{MX}_{2}$ that has a sufficiently low ionization potential. The SB height can be calculated from the numbers given in table 4.1 and figure 4.2

$$
\Phi_{\mathrm{p}}=E_{\mathrm{VB}}-W-\Delta V
$$



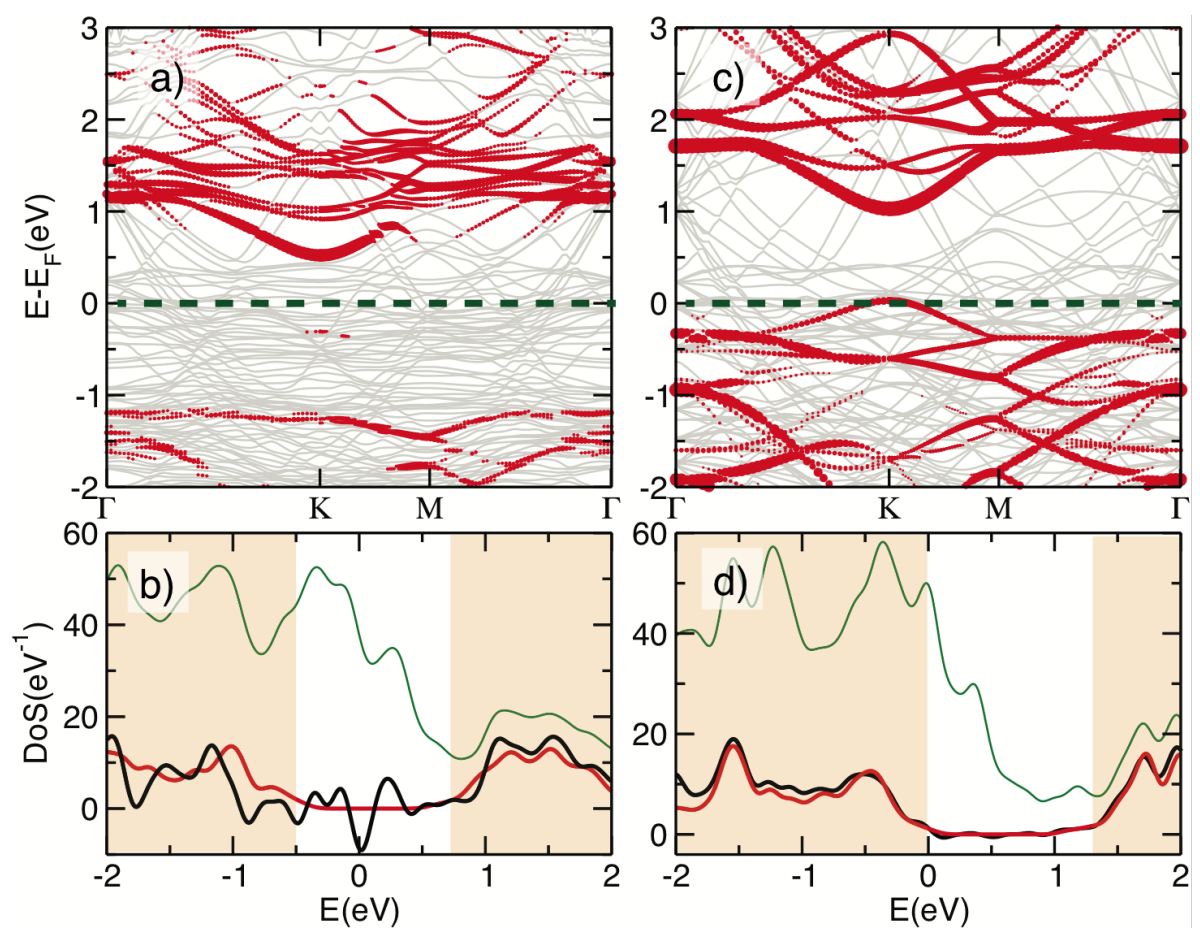

Figure 4.3: (a) Band structure (grey) of the $\mathrm{Pt}(111) / \mathrm{MoTe}_{2}$ slab; the bands are colored red according to the size of a projection of the corresponding wave functions on the $\mathrm{MoTe}_{2}$ atoms. The zero of energy is set at the Fermi level. (b) Green: total density of states, $\mathrm{DoS}_{\text {tot }}$, of the $\mathrm{Pt}(111) / \mathrm{MoTe}_{2}$ slab; black: DoS tot minus the DoS of the clean $\mathrm{Pt}(111)$ slab, $\triangle \mathrm{DoS}=\mathrm{DoS}_{\text {tot }}-\mathrm{DoS}_{\mathrm{Pt}}$; red: the DoS of a free-standing $\mathrm{MoTe}_{2}$ layer, $\mathrm{DoS}_{\mathrm{MoTe}_{2}}$. The $\mathrm{MoTe}_{2}$ band gap region is indicated in white. (c,d) Band structure and DoSs of the $\mathrm{Pt}(111) / h-\mathrm{BN} / \mathrm{MoTe}_{2}$ system.

As by definition $\Phi_{\mathrm{p}} \geq 0$, a negative number indicates a zero SB height, $\Phi_{\mathrm{p}}=0$. Only the tellurides $\mathrm{MoTe}_{2}$ and $\mathrm{WTe}_{2}$ satisfy this criterion. WSe $\mathrm{S}_{2}$ gives a small SB of 0.10 $\mathrm{eV}$ with $\mathrm{Pt} / h$-BN but the other selenide monolayers give appreciable SB heights in the range 0.2-0.5 eV. The sulfides (not shown in table 4.1) have large SBs with heights $\sim 1 \mathrm{eV}$.

One can however expect that the situation becomes more favorable for $\mathrm{MX}_{2}$ multilayers as the band gap of multilayer $\mathrm{MX}_{2}$ is smaller than that of a $\mathrm{MX}_{2}$ monolayer. For $\mathrm{MoS}_{2}$ it has been argued that the band gap reduction is monotonic in the number of layers and that it is equally divided into an upward shift of the valence band and a downward shift of the conduction band [152]. This is likely to hold more generally for all $\mathrm{MX}_{2}$ compounds. Indeed an explicit calculation of bilayer $\mathrm{WSe}_{2}$ on $\mathrm{Pt} / h$-BN gives a SB that is zero. It implies that vanishing SBs to multilayer $\mathrm{WSe}_{2}$ are possible with graphene- or $h$-BN-covered high work function metals. 


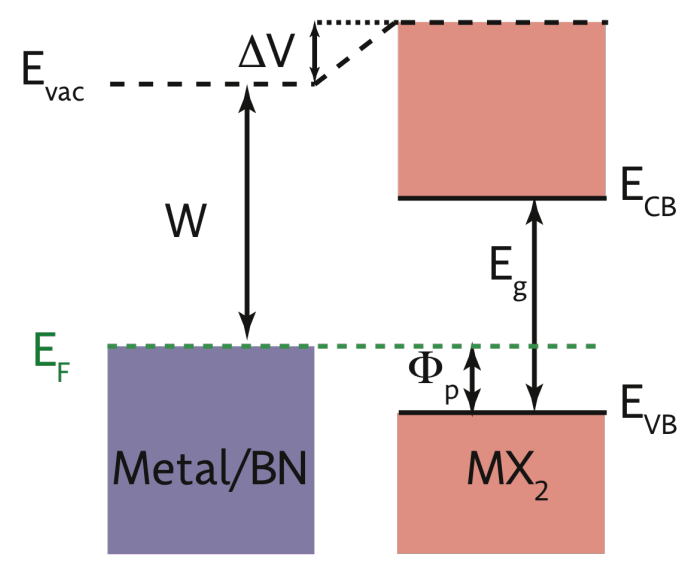

Figure 4.4: Schematic diagram defining the energy parameters used to describe the interface region in metal/ $h-\mathrm{BN} / \mathrm{MX}_{2}$ structures, with $\mathrm{E}_{\mathrm{vac}}$ and $\mathrm{E}_{\mathrm{F}}$ the vacuum level and the Fermi level, $W$ and $\Delta V$ the work function of metal $/ h$-BN and the potential step at the $h-\mathrm{BN} / \mathrm{MX}_{2}$ interface, $\mathrm{E}_{\mathrm{VB}}$ and $\mathrm{E}_{\mathrm{CB}}$, the top of the $\mathrm{MX}_{2}$ valence band and the bottom of the conduction band, $E_{g}$ the band gap, and $\Phi_{\mathrm{p}}$ the SB height for holes.

Adding additional $h$-BN layers on top of a $h$-BN/Au or $h$-BN/Pt substrate does not change the SB height, as the potential steps formed at the interfaces between the $h$-BN layers are negigibly small. By itself $h$-BN is an insulator forming SBs for holes with heights 0.9-1.1 eV and 1.1-1.3 eV with $\mathrm{Pt}$ and $\mathrm{Au}$, respectively [78]. Therefore, $h$-BN acts as a tunnel barrier between $\mathrm{Pt}$ or $\mathrm{Au}$ and $\mathrm{MX}_{2}$. A single $h$-BN layer forms only a thin barrier that is very transparent [130], but the contact resistance is expected to grow exponentionally with the number of $h$-BN layers [153].

The principles outlined above can be extended to other buffer layers besides graphene or $h$-BN. For instance, adsorbing a single $\mathrm{MoS}_{2}$ layer on a high work function metal such as Au or Pt reduces its work function by $0.5-0.7 \mathrm{eV}$. That still leaves us with a substrate that has a sufficiently high work function to yield p-type contacts to the tellurides with a zero SB height, and a small to zero SB height to the selenides, see table 4.1. As the Fermi level for $\mathrm{MoS}_{2}$ adsorbed on Au or Pt is close to the middle of the $\mathrm{MoS}_{2}$ band gap [77], the $\mathrm{MoS}_{2}$ layer then acts as tunnel barrier between the metal and the $\mathrm{MX}_{2}$ layer. Because a $\mathrm{MoS}_{2}$ monolayer is thicker than graphene or $h$ $\mathrm{BN}$, one expects it to present more of a barrier, and yield a higher contact resistance.

\subsubsection{High electron affinity oxide layers}

A buffer layer that effectively decreases a metal work function limits the scope for using it to create p-type contacts. A buffer layer that increases the work function would be more advantageous, which requires a layer that accepts electrons from the underlying metal. Oxides such as $\mathrm{MoO}_{3}$ and $\mathrm{WO}_{3}$ are known for their potential as p- 
Table 4.1: Calculated work functions $W$ of metal/graphene, metal $/ h$-BN and metal/ $\mathrm{MoS}_{2}$ substrates; potential steps $\Delta V$ at graphene/MX,$h-\mathrm{BN} / \mathrm{MX}_{2}$ and $\mathrm{MoS}_{2} / \mathrm{MX}_{2}$ interfaces; SB heights $\Phi_{\mathrm{p}}$ (eq. 4.2). All values in $\mathrm{eV}$; all $\mathrm{MX}_{2}$ in the $\mathrm{H}$ structure. The calculated work functions of clean $\mathrm{Pt}(111)$ and $\mathrm{Au}(111)$ surfaces are 6.04 and $5.58 \mathrm{eV}$, respectively.

\begin{tabular}{|c||c|cc|cc|cc|cc|}
\hline \multicolumn{1}{|c||}{} & & \multicolumn{2}{|c}{$\mathrm{MoSe}_{2}$} & \multicolumn{2}{c}{$\mathrm{WSe}_{2}$} & \multicolumn{2}{c}{$\mathrm{MoTe}_{2}$} & \multicolumn{2}{c}{$\mathrm{WTe}_{2}$} \\
\cline { 3 - 11 } & $W$ & $\Delta V$ & $\Phi_{\mathrm{p}}$ & $\Delta V$ & $\Phi_{\mathrm{p}}$ & $\Delta V$ & $\Phi_{\mathrm{p}}$ & $\Delta V$ & $\Phi_{\mathrm{p}}$ \\
\hline $\mathrm{Pt} / \mathrm{Gr}$ & 4.86 & 0.21 & 0.42 & 0.21 & 0.21 & 0.28 & 0 & 0.28 & 0 \\
$\mathrm{Pt} / h-\mathrm{BN}$ & 5.00 & 0.18 & 0.31 & 0.18 & 0.10 & 0.24 & 0 & 0.24 & 0 \\
$\mathrm{Pt} / \mathrm{MoS}_{2}$ & 5.32 & 0.14 & 0.03 & 0.14 & 0 & 0.18 & 0 & 0.18 & 0 \\
$\mathrm{Au} / \mathrm{Gr}$ & 4.80 & 0.21 & 0.48 & 0.21 & 0.27 & 0.28 & 0 & 0.28 & 0 \\
$\mathrm{Au} / h-\mathrm{BN}$ & 4.88 & 0.18 & 0.43 & 0.18 & 0.22 & 0.24 & 0 & 0.24 & 0 \\
$\mathrm{Au} / \mathrm{MoS}_{2}$ & 5.05 & 0.14 & 0.30 & 0.14 & 0.09 & 0.18 & 0 & 0.18 & 0 \\
\hline
\end{tabular}

type contacts in organic photovoltaics and light-emitting diodes $[143,144]$, and have also been tested in field-effect transistors based upn $\mathrm{MX}_{2}[145,146]$. More specifically, $\alpha-\mathrm{MoO}_{3}$ (the thermodynamically stable phase of $\mathrm{MoO}_{3}$ ) is a layered material, which consists of covalently bonded bilayers, see figure 4.1, that are van der Waals stacked. $\mathrm{MoO}_{3}$ is a large band gap material with an experimental band gap of 3.0 $\mathrm{eV}$. The electron affinity of this material is however an exceptionally high $6.7-6.9 \mathrm{eV}$ $[143,144]$. Therefore, $\mathrm{MoO}_{3}$ is predicted to be an electron acceptor with respect to common metals.

Adsorbing a $\mathrm{MoO}_{3}$-bilayer on a common metal leads to a transfer of electrons from the metal to the $\mathrm{MoO}_{3}$, and sets up a dipole layer that effectively increases the work function, provided that the adsorption process does not destroy the structure and integrity of the $\mathrm{MoO}_{3}$ overlayer. For instance, the calculated electron work function of $\mathrm{Cu}(111) /$ bilayer- $\mathrm{MoO}_{3}$ is $7.08 \mathrm{eV}$, as compared to $4.98 \mathrm{eV}$ for the clean $\mathrm{Cu}(111)$ surface. Moreover, the density of states of the $\mathrm{MoO}_{3}$ conduction band is sufficiently high such as to pin the Fermi level at the bottom of the conduction band, see figure 4.5 .

$\mathrm{A} \mathrm{MoO}_{3}$ bilayer has no dangling bonds. $\mathrm{A} \mathrm{MX}_{2}$ layer adsorbed on $\mathrm{MoO}_{3}$ is therefore likely to be van der Waals-bonded. Indeed from our calculations we find a small $\mathrm{MoO}_{3}-\mathrm{MoS}_{2}$ equilibrium binding energy of $0.17 \mathrm{eV}$ per $\mathrm{MoS}_{2}$ formula unit, and a large equilibrium bonding distance (between to top layer of oxygen atoms and the bottom layer of sulfur atoms) of $3.1 \AA$. The weak $\mathrm{MoS}_{2} / \mathrm{MoO}_{3}$ bonding has little influence on the electronic structure of either layer. Any metal that can be covered by bilayer- $\mathrm{MoO}_{3}$ without disrupting its structure should then give a work function $W$ that is sufficiently high to give a zero $\mathrm{SB}$ height to all $\mathrm{MX}$, see figure 4.2. As $W>E_{\mathrm{VB}}$ electrons are transferred from $\mathrm{MX}_{2}$ to $\mathrm{MoO}_{3}$, thereby pinning the Fermi level at the top of the $\mathrm{MX}_{2}$ valence band, as well as at the bottom of the $\mathrm{MoO}_{3}$ conduction band, see figure 4.5 .

The $\mathrm{MoO}_{3}$ layer does not act as a tunnel barrier, as transport of charge carriers 


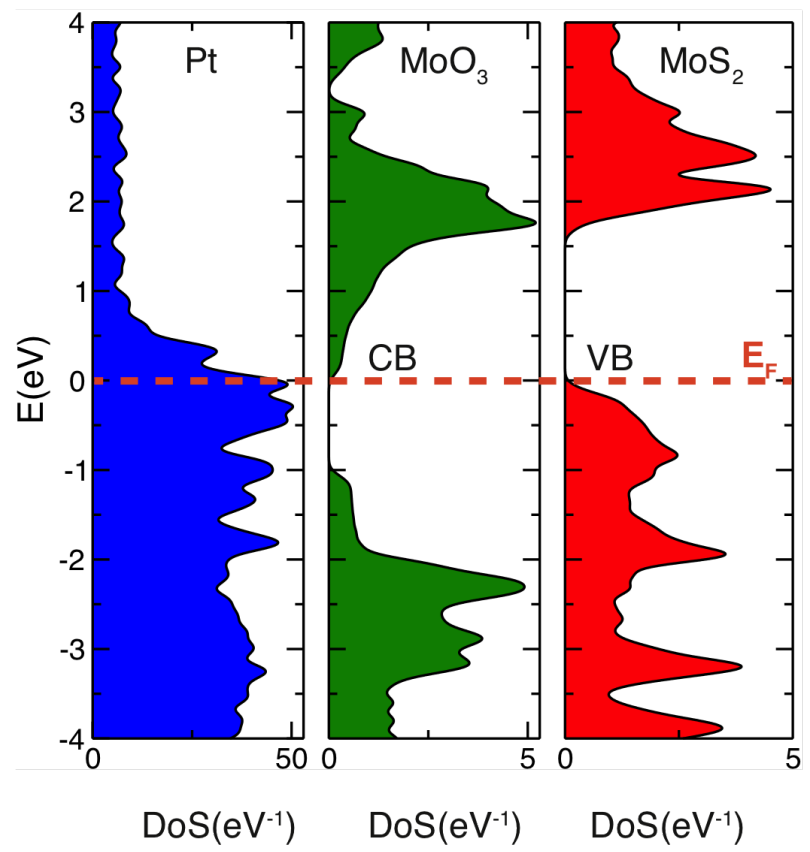

Figure 4.5: Densities of states of $\mathrm{Pt}(111)$ (left), bilayer- $\mathrm{MoO}_{3}$ (middle), and $\mathrm{MoS}_{2}$ (right). In a Pt/bilayer- $\mathrm{MoO}_{3} / \mathrm{MoS}_{2}$ multilayer the Fermi level $E_{\mathrm{F}}$ is pinned at the bottom of the $\mathrm{MoO}_{3}$ conduction band $E_{\mathrm{CB}}$ and at the top of the $\mathrm{MoS}_{2}$ valence band $E_{\mathrm{VB}}$.

takes place through the conduction band of the oxide. If one adds additional $\mathrm{MoO}_{3}$ layers, one expects that for thicknesses below the mean free path of the charge carriers the characteristics for injection from the metal into the $\mathrm{MX}_{2}$ layer remain the same. The contact resistance does increase for thicker $\mathrm{MoO}_{3}$ layers, however, as charge carrier mobilities in oxide layers are typically substantially smaller than in metals [145].

In principle any oxide that has a layered structure can be used this way. One can cover a metal with an oxide monolayer; if the oxide layer bonds to $\mathrm{MX}_{2}$ through van der Waals interactions, and if its electron affinity is sufficiently high, it should give a p-type contact. In contrast to graphene or $h$-BN, the metal species is not very important, as the conduction band of the oxide pins the Fermi level. Besides $\mathrm{MoO}_{3}$, for instance $\mathrm{V}_{2} \mathrm{O}_{5}$ has a layered structure and a high electron affinity of up to $7 \mathrm{eV}$ [143]. A drawback of using such oxide layers are that they are strong oxidizing agents, which can react with molecules in the environment, or with the metal electrodes. For instance, the $\mathrm{Cu} / \mathrm{MoO}_{3}$ interface is metastable and oxidized $\mathrm{Cu}$ ions can diffuse into $\mathrm{MoO}_{3}$ [143]. Other metal/ $\mathrm{MoO}_{3}$ interfaces, such as $\mathrm{Au} / \mathrm{MoO}_{3}$, are stable, however. 


\subsubsection{Metallic $\mathrm{M}^{\prime} \mathrm{X}_{2}^{\prime}$ buffer layers}

A buffer with a high electron affinity that is less reactive than an oxide would be very convenient. Metallic $\mathrm{M}^{\prime} \mathrm{X}_{2}^{\prime}, \mathrm{M}^{\prime}=\mathrm{V}, \mathrm{Nb}, \mathrm{Ta}, \mathrm{X}^{\prime}=\mathrm{S}$, Se compounds have a layered hexagonal structure similar to that of semiconducting $\mathrm{MX}_{2}$. The latter compounds contain a group VI transition metal M, whereas the former compounds contain a group $\mathrm{V}$ transition metal $\mathrm{M}^{\prime}$. The electronic structure of these two compound groups is basically similar, but in $\mathrm{MX}_{2}$ the topmost valence band is completely filled, whereas in $\mathrm{M}^{\prime} \mathrm{X}_{2}^{\prime}$ it is only half-filled because $\mathrm{M}^{\prime}$ has one electron less than $\mathrm{M}$ [151]. This makes the $\mathrm{M}^{\prime} \mathrm{X}_{2}^{\prime}$ compounds metallic with a relatively high work function. For example, $\mathrm{NbS}_{2}$ and $\mathrm{TaS}_{2}$ monolayers have calculated work functions of 6.22 and 6.12, respectively.

Most common metals have a lower work function. If they are covered by a $\mathrm{M}^{\prime} \mathrm{X}_{2}^{\prime}$ monolayer, electrons are transferred from the metal to the $\mathrm{M}^{\prime} \mathrm{X}_{2}^{\prime}$ layer, effectively increasing the work function, as in the case of an $\mathrm{MoO}_{3}$ overlayer. As the density of states at the Fermi level of $\mathrm{M}^{\prime} \mathrm{X}_{2}^{\prime}$ is high, the transferred electrons will hardly modify the Fermi level, which is therefore effectively pinned by the $\mathrm{M}^{\prime} \mathrm{X}_{2}^{\prime}$ layer. For instance, the $\mathrm{Au}(111)$ and $\mathrm{Al}(111)$ surfaces have calculated work functions of 5.58 and $4.24 \mathrm{eV}$, respectively. Adsorbing a $\mathrm{NbS}_{2}$ monolayer gives work functions of $\mathrm{Au} / \mathrm{NbS}_{2}$ and $\mathrm{Al} / \mathrm{NbS}_{2}$ surfaces of 6.11 and $6.06 \mathrm{eV}$, respectively. In other words, the work function differs by $\lesssim 0.15 \mathrm{eV}$ from that of a free-standing $\mathrm{NbS}_{2}$ monolayer, irrespective of the metal substrate.

This remarkable property make metallic $\mathrm{M}^{\prime} \mathrm{X}_{2}^{\prime}$ compounds viable buffer layers for making $\mathrm{p}$-doped contacts to $\mathrm{MX}_{2}$ semiconductors. We envision that $\mathrm{M}^{\prime} \mathrm{X}_{2}^{\prime}$ layers can be deposited on a metal substrate in a similar way as $\mathrm{MX}_{2}$ layers. $\mathrm{A} \mathrm{MX}_{2}$ layer can then be deposited on $\mathrm{M}^{\prime} \mathrm{X}_{2}^{\prime}$ by van der Waals epitaxy, for instance $[134,135]$. The interaction at the $\mathrm{M}^{\prime} \mathrm{X}_{2}^{\prime} / \mathrm{MX}_{2}$ interface is van der Waals, which hardly perturbs the electronic structure of either layer. The potential step at the $\mathrm{M}^{\prime} \mathrm{X}_{2}^{\prime} / \mathrm{MX}_{2}$ interface, $\Delta V \approx 0.1 \mathrm{eV}$, is small and hardly affects the $\mathrm{M}^{\prime} \mathrm{X}_{2}^{\prime}$ work function.

Figure 4.6 shows the calculated band structure of the $\mathrm{Au}(111) / \mathrm{NbS}_{2} / \mathrm{WSe}_{2}$ multilayer. It illustrates the perfect p-type contact formed in this case, with the Fermi level coinciding with the top of the $\mathrm{WSe}_{2}$ valence band. At the same time the Fermi cuts the valence band of $\mathrm{NbS}_{2}$, confirming that it acts as a conducting layer. Indeed, the local DoS calculated at the Fermi energy shows a state that is delocalized over the whole multilayer, see figure 4.6. We expect that similar p-type contacts can be formed with other $\mathrm{MX}_{2}$ layers using $\mathrm{NbS}_{2}$ or $\mathrm{TaS}_{2}$ monolayers as a buffer.

Adding additional $\mathrm{NbS}_{2}$ or $\mathrm{TaS}_{2}$ layers, the charge carrier injection into $\mathrm{MX}_{2}$ should remain the same as long as the thickness of the buffer layers is below the mean free path of the charge carriers. For thicker layers the contact resistance starts to depend on the thickness of the buffer layers. 

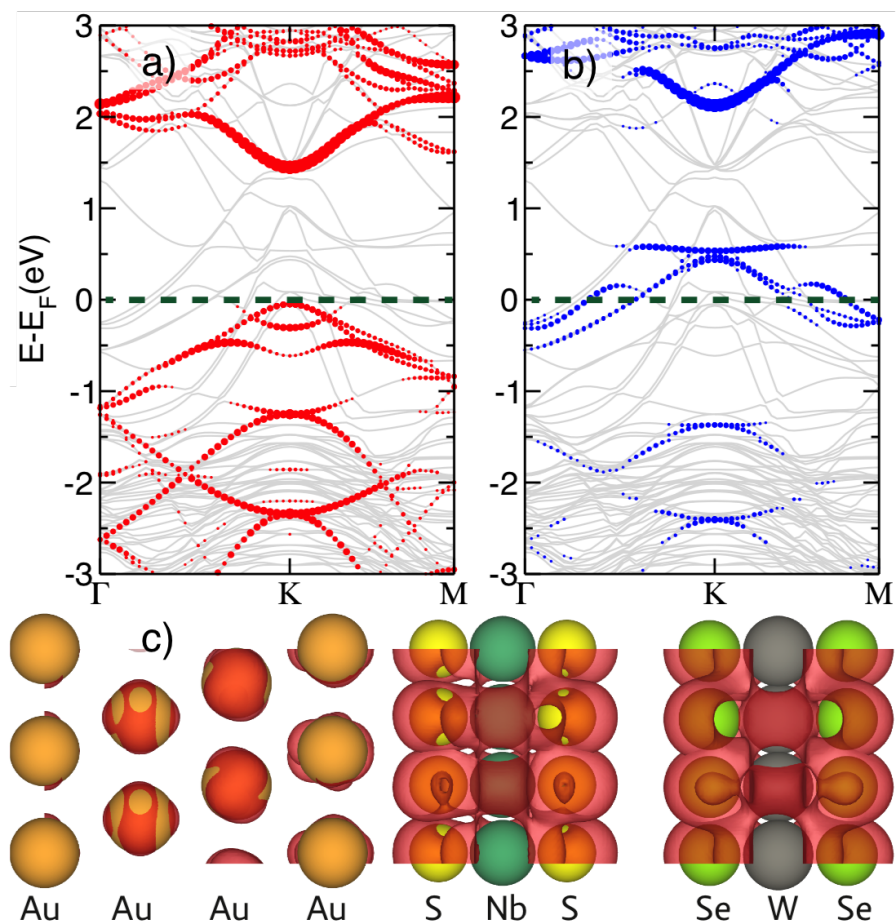

Figure 4.6: $(\mathrm{a}, \mathrm{b})$ Band structure of $\mathrm{Au}(111) / \mathrm{NbS}_{2} / \mathrm{WSe}_{2}$. The red and blue colors indicate projections of the wave functions on the $\mathrm{WSe}_{2}$ and the $\mathrm{NbS}_{2}$ atoms, respectively. The zero of energy is set at the Fermi level. (c) Local density of states calculated in an energy interval of $0.1 \mathrm{eV}$ around the Fermi level.

\subsection{Conclusions}

We propose a particular technique to construct p-type contacts with zero SB heights to $\mathrm{MX}_{2}(\mathrm{M}=\mathrm{Mo}, \mathrm{W} ; \mathrm{X}=\mathrm{S}, \mathrm{Se}, \mathrm{Te}) 2 \mathrm{D}$ semiconductors. Using first-principles DFT calculations we show that a direct metal $/ \mathrm{MX}_{2}$ interaction leads to interface states that pin the Fermi level in the $\mathrm{MX}_{2}$ band gap, giving a sizable SB. Inserting a well-chosen buffer layer between the metal surface and the $\mathrm{MX}_{2}$ layer breaks this interaction, and unpins the Fermi level, if $\mathrm{MX}_{2}$ is bonded to the buffer layer with van der Waals forces.

A monolayer of $h$-BN or graphene constitute only a thin barrier for transport. Adsorbing $h$-BN on a high work function metal such as Pt or Au, gives a zero SB height for contacts to the $\mathrm{MX}_{2}$ tellurides, a low SB to $\mathrm{WSe}_{2}$, and zero SB heights for contacts to telluride and selenide multilayers. Adsorbing graphene works in a similar way, but is somewhat less effective in reducing SBs. To obtain p-type contacts one has to combine these layers with high work function metals, as adsorption of $h$ $\mathrm{BN}$ or graphene substantially reduces the work function of the metal substrate. 
Alternatively one can use a buffer layer of an oxide with a high electron affinity. By explicit calculations on metal/ $\mathrm{MoO}_{3}$ we show that adsorbing a bilayer of $\mathrm{MoO}_{3}$ gives a zero $\mathrm{SB}$ height for contacts to all $\mathrm{MX}_{2}$. The metal substrate is relatively unimportant here, as the Fermi level is pinned at the $\mathrm{MoO}_{3}$ conduction band, which also ensures that bilayer- $\mathrm{MoO}_{3}$ does not act as a tunnel barrier to $\mathrm{MX}$. Bilayer- $\mathrm{MoO}_{3}$ binds to $\mathrm{MX}_{2}$ layers via van der Waals interactions, consistent with the principal idea of this paper.

Strong oxididants such as $\mathrm{MoO}_{3}$ might suffer from chemical instability. The most elegant solution for the p-type contact problem is using a metallic $\mathrm{M}^{\prime} \mathrm{X}_{2}^{\prime}(\mathrm{M}=\mathrm{Nb}$, Ta; $\mathrm{X}=\mathrm{S}$ ) monolayer as a buffer. These compounds are stable and have a high work function. Adsorbed on a metal substrate they yield a high work function contact, which gives a zero $\mathrm{SB}$ height to all $\mathrm{MX}_{2}$ monolayers. The metal substrate is of little importance, as the Fermi level is pinned by the $\mathrm{M}^{\prime} \mathrm{X}_{2}^{\prime}$ layer. $\mathrm{MX}_{2}$ forms a van der Waals bonded contact to $\mathrm{M}^{\prime} \mathrm{X}_{2}^{\prime}$, and the states formed at the Fermi level extend throughout the whole metal $/ \mathrm{M}^{\prime} \mathrm{X}_{2}^{\prime} / \mathrm{MX}_{2}$ multilayer.

\subsection{Computational section}

We perform first-principles density functional theory (DFT) calculations using the VASP code and the projector augmented wave (PAW) datasets from the VASP database [44, 45, 46, 47]. For the computational set-up and the choice of computational parameters (unit cell, $k$-point sampling, etc.) we follow Refs. [77] and [154].

Obviously different 2D materials have different in-plane lattice constants. Van der Waals stacking of 2D materials then generally leads to incommensurable structures, which are observed experimentally in the form of moiré patterns. In electronic structure calculations one is forced to approximate these by commensurable structures, which introduces an artificial strain. As the $\mathrm{MX}_{2}$ electronic structure is very sensitive to strain, we keep the in-plane lattice constant of $\mathrm{MX}_{2}$ at its optimized value, adapting the lattice constant of the metal surfaces and the buffer layers accordingly. The supercells defining the commensurable structures are constructed such that the lattice mismatches between the different layers are minimal, following the technique explained in Ref. [154]. As an example, a $(2 \times 2) \mathrm{MoTe}_{2}$ cell on top of $(3 \times 3) h$-BN on $(\sqrt{7} \times \sqrt{7}) \mathrm{Pt}$ gives lattice mismatches of 6 and $4 \%$ in the $h$-BN and $\mathrm{Pt}$ lattices respectively.

Van der Waals interactions are vital to descibe the bonding between the layers. Here we use the van der Waals density functional opt86b-vdW-DF [63, 64, 65]. The optimized lattice parameters $a$ of $\mathrm{MX}_{2}$ monolayers are in good agreement with the experimental lattice parameters of the bulk hexagonal structures, see table 4.2. (The experimental structure of $\mathrm{WTe}_{2}$ is not hexagonal [161]; it has been added for completeness reasons.) Also in table 4.2 are listed the calculated monolayer band gaps $E_{g}$. DFT calculations usually severely underestimate band gaps, but for $\mathrm{MX}_{2}$ monolayers the differences are tolerable, if one compares to the measured optical gaps $E_{\mathrm{opt}}^{\exp }$ of the monolayers, see table 4.2. 
Table 4.2: Optimized in-plane lattice constant $a(\AA)$ and calculated band gap $E_{g}(\mathrm{eV})$ of the $\mathrm{H}-\mathrm{MX}_{2}$ monolayers. All calculated values are obtained using opt86b-vdw-DF.

\begin{tabular}{|c|c|c|c|c|c|c|c|}
\hline & $\mathrm{MoS}_{2}$ & $\mathrm{MoSe}_{2}$ & $\mathrm{MoTe}_{2}$ & $\mathrm{WS}_{2}$ & $\mathrm{WSe}_{2}$ & $\mathrm{WTe}_{2}$ & $\mathrm{NbS}_{2}$ \\
\hline$a$ & 3.16 & 3.29 & 3.52 & 3.16 & 3.29 & 3.52 & 3.31 \\
\hline$a_{\exp }^{\text {a) }}$ & 3.16 & 3.30 & 3.52 & 3.15 & 3.28 & - & 3.31 \\
\hline$E_{g}$ & 1.76 & 1.50 & 1.13 & 1.89 & 1.62 & 1.13 & - \\
\hline$E_{\mathrm{opt}}^{\exp }$ & $1.86^{\mathrm{b}), \mathrm{c}), \mathrm{d})}$ & $1.63^{d), e)}$ & $1.10^{\mathrm{f})}$ & $1.99 \mathrm{~g})$ & $\left.1.65^{b}\right)$ & - & - \\
\hline
\end{tabular}

Such a comparison is not entirely fair, as $E_{g}-E_{\mathrm{opt}}^{\exp }$ is the exciton binding energy. The latter depends strongly on the environment of the exciton, and decreases if the electrodynamic screening by the environment increases [116]. Values of the exciton binding energy between zero [25, 162], and $0.5 \mathrm{eV}[115,158]$ have been reported. In our case one expects the screening to be strong as the $\mathrm{MX}_{2}$ layers are close to a metal substrate, and we speculate the exciton binding energy to be relatively small.

Comparing to previous calculations, one observes that the opt86b-vdw-DF functional gives an improvement over the GGA/PBE functional regarding optimized lattice constants and band gaps of $\mathrm{MX}_{2}$ [163]. The opt86b-vdw-DF lattice constants are close to the HSE06 values, and the band gaps are in between the GGA/PBE and HSE06 values. Concerning the absolute positions of the energy levels, the valence band maxima $E_{\mathrm{VB}}$ calculated with opt86b-vdw-DF (figure 4.1) and HSE06 [163] are generally with $\sim 0.15 \mathrm{eV}$ of one another. It is difficult to compare these values to experimental data. Note however that the experimentally determined valence band offset of $0.83 \mathrm{eV}$ between $\mathrm{MoS}_{2}$ and $\mathrm{WSe}_{2}$ [158], is in excellent agreement with the $0.83 \mathrm{eV}$ derived from the numbers in figure 4.1 . 


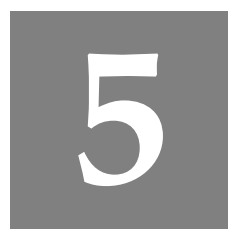

\section{Green's function approach to edge states in transition metal dichalcogenides}

Two-dimensional semiconductors such as transition metal dichalcogenides often show an abundance of one-dimensional metallic edges and grain boundaries. Standard techniques for calculating edge states typically model nanoribbons, and require the use of large supercells. In this paper we formulate a Green's function technique for calculating edge states of (semi-)infinite two-dimensional systems with a single well-defined edge or grain boundary. We express Green's functions in terms of Bloch matrices, constructed from the solutions of a quadratic eigenvalue equation. The technique can be applied to any localized basis representation of the Hamiltonian. Here we use it to calculate edge states of $M X_{2}$ monolayers by means of tight-binding models. Besides the basic zigzag and armchair edges, we study edges with a more general orientation, structurally modifed edges, and grain boundaries.

\subsection{Introduction}

In the wake of graphene a whole new class has emerged of materials that are essentially two-dimensional (2D) in nature [4, 164]. The subset of materials with honeycomblike structures alone contains metals such as graphene, insulators such as boron nitride $(h-\mathrm{BN})$, and semiconductors such as the transition metal dichalcogenides $\left(\mathrm{MX}_{2} ; \mathrm{M}=\mathrm{Mo}, \mathrm{W}, \mathrm{X}=\mathrm{S}, \mathrm{Se}, \mathrm{Te}\right)[21,68]$. It becomes more and more feasible to grow nanostructures and in-plane heterostructures of $2 \mathrm{D}$ materials in a controlled way $[165,166,167,168,169,170,171]$. As a result the electronic structure of edges and grain boundaries attracts increasing attention.

Graphene edges, for instance, are predicted to have remarkable one-dimensional 
electronic and magnetic properties $[172,173]$. The edges and grain boundaries of $\mathrm{MX}_{2}$ sheets are generally metallic [170,174, 175, 176, 177, 178, 179, 180]. As the bulk $2 \mathrm{D} \mathrm{MX}$ materials are semiconducting, the metallicity is truly localized at the edge or grain boundary, giving access to one-dimensional (1D) physics. In chemistry $\mathrm{MoS}_{2}$ edges have been identified as sites that show a special catalytic activity $[174,176,181]$. Such experimental developments have motivated a large number of calculations on the electronic structure of edge states, both first-principles calculations, as well as model calculations.

The electronic structure of graphene edges in particular have been studied extensively. As graphene has a relatively simple electronic structure, some features of the edge states in graphene can be studied by analytical or simple numerical techniques $[182,183,184]$. The edges of $\mathrm{MoS}_{2}$ have also attracted special attention $[174,175,176,177,178,185,186,179,187,188,94,189,180,190,191,192]$. Here the complexity of the electronic structure requires more extensive calculations, even for relatively simple modeling at the tight-binding level. A standard technique for calculating edge states uses supercells to model nanoribbons of a finite width. Drawbacks of this approach are that the electronic structure of the edge states is mixed in with that of the bulk-like interior of the nanoribbon, and that the two edges of the nanoribbon can interact electronically. The ribbon has therefore to be sufficiently wide in order to electronically separate the two edges from one another and from the bulk. It requires the use of large supercells, which in particular if the materials are modeled from first principles, leads to time-consuming calculations and analysis of the results.

Green's function techniques constitute an alternative approach. They enable calculations on semi-infinite structures with a single well-defined edge or infinite structures around a grain boundary, and they generally do not require the use of large supercells. Green's function techniques have been formulated and explored for calculations of surface states of 3D materials [193, 194, 195, 196, 197]. Here we formulate a special Green's function technique for calculating edge and grain boundary states. This technique is inspired by the Green's function formalism that has been introduced for calculating electronic transport through nanostructures [198, 199, 200, 201, 202].

We express both edge Green's functions and bulk Green's functions in terms of Bloch matrices, which are constructed from the eigenvalues and eigenfunctions of a quadratic eigenvalue problem [198, 199, 200, 203, 201, 202]. Structural and chemical modifications at the edges and grain boundaries are then tackled by connecting the modified edges to semi-infinite bulk structures. The method allows for a clean separation of edge and bulk properties at a moderate computational cost.

Our Green's function approach requires a representation of the Hamiltonian on a localized basis, such as atomic orbitals or Wannier functions, or a real space grid. It can be applied to tight-binding, as well as first-principles models. In this paper we illustrate its use on tight-binding models for $\mathrm{MX}_{2}$ monolayers, $\mathrm{MoS}_{2}$ in particular. We apply the approach to the three-band model for the simplified electronic 
structure of $\mathrm{MoS}_{2}$, developed by Mattheis [151] and Liu et al. [204]. We study the electronic structures of the elementary (zigzag, armchair) $\mathrm{MoS}_{2}$ edges, and of edges of a more general orientation. We illustrate the effect on the electronic structure of edge modifications and of the formation of grain boundaries. For comparison we also study edges within an eleven-band model comprising all $\mathrm{MoS}_{2}$ valence orbitals, developed by Rostami et al. [205] and in the present paper.

The paper is organized as follows. In Sec. 5.2.1 we formulate the technique of calculating Green's functions by Bloch matrices. How to apply this technique to $\mathrm{MX}_{2}$ edges and grain boundaries is described in Sec. 5.2.2. The specific tight-binding models are discussed in Sec. 5.2.3, and appendices A.1 and A.2. We discuss results obtained with these models for $\mathrm{MX}_{2}$ edges and grain boundaries in Secs. 5.3.1 and 5.3.2.

\subsection{Theory}

\subsubsection{Green's functions}

We divide the 2D layers into 1D strips, see Fig. 5.1 [195]. Assuming translational symmetry with period $a$ along the strip, the Hamiltonian matrix can be labeled by a Bloch wave number $-\frac{\pi}{a}<k \leq \frac{\pi}{a}$. For clarity of notation we suppress the label $k$ in the following. The thickness of the strips is chosen such that a direct interaction exists between neighboring strips only. We label the strips by an index $i$, and divide the Hamiltonian matrix into blocks $\mathbf{H}_{i, j}$, with an $i, j=-\infty, \ldots, \infty$ for an inifinite system. Having only nearest neighbor interactions between strips means that the Hamiltonian matrix is block tridiagonal, i.e., $\mathbf{H}_{i, j}=0 ; j \neq i, i \pm 1$. For a unit cell in the strip containing $N$ orbitals, all these matrix blocks are $N \times N$.

The columns of the retarded Green's function matrix blocks $\mathbf{G}_{i, j}$ obey

$$
-\mathbf{H}_{i, i-1} \mathbf{G}_{i-1, j}+\left(E^{+} \mathbf{I}-\mathbf{H}_{i, i}\right) \mathbf{G}_{i, j}-\mathbf{H}_{i, i+1} \mathbf{G}_{i+1, j}=\mathbf{I} \delta_{i j}
$$

where $\mathbf{I}$ is the $N \times N$ identity matrix, and $E^{+}=E+i \eta$ with $\eta$ the usual infinitesimal positive real number. Note that we assume a representation based upon an orthogonal basis set. We are foremost interested in the layer resolved density of states, given by the usual expression $n_{i}=-\pi^{-1} \operatorname{Im} \operatorname{Tr} \mathbf{G}_{i, i}$. Obviously, besides on the Bloch wave number $k$, the Green's function matrix also depends on the energy $E$. Again for ease of notation we often omit both these labels in the following.

In an infinite system with translational symmetry between the layers, the strips are identical, and Eq. 5.1 then becomes

$$
-\mathbf{B G}_{i-1, j}+\left(E^{+} \mathbf{I}-\mathbf{H}\right) \mathbf{G}_{i, j}-\mathbf{B}^{\dagger} \mathbf{G}_{i+1, j}=\mathbf{I} \delta_{i j},
$$

with $\mathbf{B}=\mathbf{H}_{i, i-1}, \mathbf{H}=\mathbf{H}_{i, i}$, and $\mathbf{B}^{\dagger}=\mathbf{H}_{i, i+1}$ for $i=-\infty, \ldots, \infty$, see Fig. 5.1. For a left semi-infinite system with $i=-\infty$ to $i=0$, the equations remain the same, 


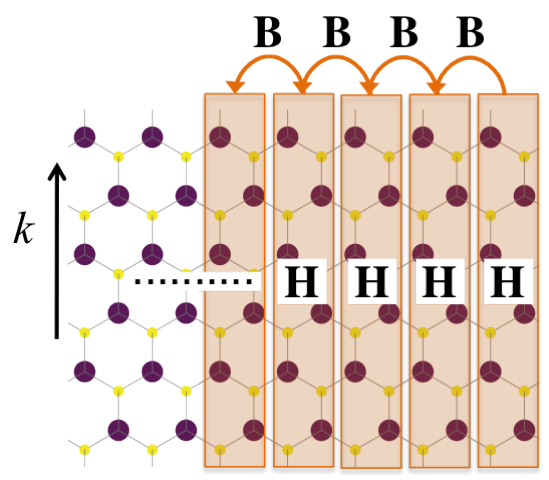

Figure 5.1: (Color online) A 2D layer is divided in 1D strips. Translation symmetry along the strips gives the Bloch wave number $k$. Translation symmetry between the strips results in identical on-strip Hamiltonian matrix blocks $\mathbf{H}(k)$ and hopping matrix blocks $\mathbf{B}(k)$.

except for the $i=0$ equation, which becomes

$$
-\mathbf{B G}_{-1, j}^{L}+\left(E^{+} \mathbf{I}-\mathbf{H}\right) \mathbf{G}_{0, j}^{L}=\mathbf{I} \delta_{0 j} .
$$

In surface science the matrix block $\mathbf{g}_{L}=\mathbf{G}_{0,0}^{L}$ is called the surface Green's function. In the context of 2D structures we call it the edge Green's function.

In a similar way, for a right semi-infinite system with $i=0, \infty$, only the equation for $i=0$ is different from the bulk equation, Eq. 5.2

$$
\left(E^{+} \mathbf{I}-\mathbf{H}\right) \mathbf{G}_{0, j}^{R}-\mathbf{B}^{\dagger} \mathbf{G}_{1, j}^{R}=\mathbf{I} \delta_{0 j},
$$

with the matrix block $\mathbf{g}_{R}=\mathbf{G}_{0,0}^{R}$ the edge Green's function. Note that if the 2D layer has no two-fold symmetry, such as inversion, a mirror plane perpendicular to the $2 \mathrm{D}$ layer, or a two-fold rotation axis perpendicular to the $2 \mathrm{D}$ layer, then $\mathbf{g}_{R} \neq \mathbf{g}_{L}$, i.e., a right edge is different from a left edge. by

The density of states of the edge strip of a left/right semi-infinite system is given

$$
n_{L / R}^{(S)}=\sum_{\alpha=1}^{N} n_{\alpha} ; \quad n_{\alpha}=-\pi^{-1} \operatorname{Im}\left[\mathbf{g}_{L / R}\right]_{\alpha, \alpha},
$$

where $n_{\alpha}$ is the density of states projected on a single orbital $\alpha$.

Partitioning the infinite system into a right and a left semi-infinite halve, the onstrip matrix blocks of the Green's function of the infinite system can be expressed as

$$
\mathbf{G}_{i, i}=\left[\mathbf{g}_{L}^{-1}-\mathbf{B}^{\dagger} \mathbf{g}_{R} \mathbf{B}\right]^{-1} .
$$




\section{Eigenmodes and Bloch matrices}

We will express the Green's function matrices of (semi)infinite systems in terms of Bloch matrices [198]. Equation 5.2 for $i \neq j$ is the same as the Schrödinger equation in tight-binding representation

$$
-\mathbf{B} \mathbf{c}_{i-1}+(E \mathbf{I}-\mathbf{H}) \mathbf{c}_{i}-\mathbf{B}^{\dagger} \mathbf{c}_{i+1}=0,
$$

where $\mathbf{c}_{i}$ is the $N$-dimensional vector of orbital coefficients of the wave function on strip $i$. With translational symmetry between the strips, the elementary solution is a Bloch wave, and $\mathbf{c}_{i+1}=\lambda \mathbf{c}_{i}$, with $\lambda$ a complex constant. For an inifinite system this holds for all $i$, and for a left (right) semi-infinite system for $i<0(i>0)$. Using this relation as an ansatz in Eq. 5.7 gives a quadratic eigenvalue equation in $\lambda$ of dimension $N$.

$$
\mathbf{A}(\lambda) \mathbf{u}=0 ; \quad \mathbf{A}(\lambda)=-\mathbf{B}+\lambda(E \mathbf{I}-\mathbf{H})-\lambda^{2} \mathbf{B}^{\dagger} .
$$

As $\mathbf{A}(\lambda)=\lambda^{2} \mathbf{A}^{\dagger}\left(1 / \lambda^{*}\right)$, if $\lambda$ is a root of $\operatorname{det} \mathbf{A}(\lambda)$, then so is $1 / \lambda^{*}$. Numerically, the solutions are usually found by solving an equivalent linear generalized eigenvalue equation of dimension $2 N$ [203],

$$
\left[\left(\begin{array}{cc}
\mathbf{I} & \mathbf{0} \\
E \mathbf{I}-\mathbf{H} & -\mathbf{B}
\end{array}\right)-\lambda\left(\begin{array}{cc}
\mathbf{0} & \mathbf{I} \\
\mathbf{B}^{\dagger} & \mathbf{0}
\end{array}\right)\right] \mathbf{z}=\mathbf{0}
$$

which resembles the eigenvalue equation for the transfer matrix [195, 196, 206, 207, 208, 209].

The maximally $2 N$ solutions of this equation can be divided into two classes, i.e. right-going modes and left-going modes, labeled respectively by + and - superscripts in the following. Right-going modes are either evanescent waves that decay to the right, $\left|\lambda_{n}^{+}\right|<1$, or waves that travel to the right, meaning $\left|\lambda_{n}^{+}\right|=1$ and a positive group velocity. Left-going modes either decay to the left, $\left|\lambda_{n}^{-}\right|>1$, or travel to the left, $\left|\lambda_{n}^{-}\right|=1$ with negative group velocity. With $\mathbf{u}_{n}$ the eigenvector belonging to the eigenvalue $\lambda_{n}$ in Eq. 5.9, the group velocity is given by [200]

$$
v_{n}=-\frac{2 a}{\hbar} \operatorname{Im}\left[\lambda_{n} \mathbf{u}_{n}^{\dagger} \mathbf{B}^{\dagger} \mathbf{u}_{n}\right],
$$

Only for traveling waves is the group velocity non-zero.

We divide the eigenvectors into a set of $N^{+}$right-going modes $\mathbf{u}_{n}^{+}$and a set of $N^{-}$ left-going modes $\mathbf{u}_{n}^{-}$. The evanescent waves always come in pairs of a right-going and a left-going mode, i.e., if $\lambda_{n}^{+}$is the eigenvalue of a right-going mode, then $\lambda_{n}^{-}=$ $1 /\left(\lambda_{n}^{+}\right)^{*}$ gives a left-going solution. Traveling waves do not necessarily come in such pairs, and the numbers of right- and left-going traveling waves may be different. Neither right-going modes or left-going modes necessarily form a complete set in $\mathrm{N}$-dimensional space, nor are they an orthogonal set in general. 
One can use these two sets of modes to form the two $N \times N^{ \pm}$matrices

$$
\mathbf{U}^{ \pm}=\left(\mathbf{u}_{1}^{ \pm}, \mathbf{u}_{1}^{ \pm}, \ldots, \mathbf{u}_{N^{ \pm}}^{ \pm}\right)
$$

and construct the two Bloch matrices

$$
\mathbf{F}^{ \pm}=\mathbf{U}^{ \pm} \boldsymbol{\Lambda}^{ \pm} \widetilde{\mathbf{U}}^{ \pm}
$$

where $\Lambda^{ \pm}$are the $N^{ \pm} \times N^{ \pm}$diagonal matrices with the eigenvalues $\lambda_{n}^{ \pm}$on the diagonal, and $\widetilde{\mathbf{U}}^{ \pm}$are the $N^{ \pm} \times N$ (pseudo)inverses of $\mathbf{U}^{ \pm}$.[210] The Bloch matrices have the convenient property

$$
\left(\mathbf{F}^{ \pm}\right)^{p}=\mathbf{U}^{ \pm}\left(\boldsymbol{\Lambda}^{ \pm}\right)^{p} \widetilde{\mathbf{U}}^{ \pm}
$$

for any integer $p$, as $\widetilde{\mathbf{U}^{ \pm}} \mathbf{U}^{ \pm}=\mathbf{I}_{M^{ \pm}}$, the $M^{ \pm} \times M^{ \pm}$identity matrix, with $M^{ \pm} \leq N^{ \pm}$. It follows that

$$
\mathbf{c}_{i}=\left(\mathbf{F}^{+}\right)^{i} \mathbf{c}_{0}^{+}+\left(\mathbf{F}^{-}\right)^{i} \mathbf{c}_{0}^{-},
$$

satisfies the tight-binding equation, Eq. 5.7, where $\mathbf{c}_{0}^{ \pm}$set the boundary conditions in strip number 0 . We assume that all relevant solutions can be expressed this way.

\section{Green's functions in terms of Bloch matrices}

The general expression of Eq. 5.14 also applies to the columns of the Green's function matrices, compare Eqs. 5.2 and 5.7. The boundary conditions require that a retarded Green's function comprises traveling waves moving outwards from its point source and/or evanescent waves decaying away from the source. For left and right semiinfinite systems this gives

$$
\begin{aligned}
\mathbf{G}_{i, 0}^{L} & =\left(\mathbf{F}^{-}\right)^{i} \mathbf{g}_{L}, i<0, \\
\mathbf{G}_{i, 0}^{R} & =\left(\mathbf{F}^{+}\right)^{i} \mathbf{g}_{R}, i>0 .
\end{aligned}
$$

Using Eq. 5.15 in Eqs. 5.2 and 5.3 then leads to

$$
\begin{aligned}
& {\left[-\mathbf{B}\left(\mathbf{F}^{-}\right)^{-1}+E^{+} \mathbf{I}-\mathbf{H}\right] \mathbf{g}_{L}=\mathbf{I},} \\
& {\left[-\mathbf{B}\left(\mathbf{F}^{-}\right)^{-1}+E^{+} \mathbf{I}-\mathbf{H}-\mathbf{B}^{\dagger} \mathbf{F}^{-}\right]\left(\mathbf{F}^{-}\right)^{-1} \mathbf{g}_{L}=\mathbf{0} .}
\end{aligned}
$$

Solving these two equations gives the edge Green's function of a left semi-infinite system as

$$
\mathbf{g}_{L}=\left[\mathbf{B}^{\dagger} \mathbf{F}^{-}\right]^{-1}
$$

where the inversion should be treated as a pseudoinversion if $\mathbf{B}^{\dagger}$ is singular.[210] Using Eq. 5.16 in Eqs. 5.2 and 5.4 gives the edge Green's function of a right semiinfinite system

$$
\mathbf{g}_{R}=\left[\mathbf{B}\left(\mathbf{F}^{+}\right)^{-1}\right]^{-1}
$$


Finally, using Eq. 5.6 gives the on-strip Green's function matrix block of an infinite system

$$
\mathbf{G}_{i, i}=\left[\mathbf{B}^{\dagger} \mathbf{F}^{-}-\mathbf{B}^{\dagger} \mathbf{F}^{+}\right]^{-1}
$$

\section{Ideal edge states}

One cannot have traveling Bloch waves for energies in the band gap of a semiconductor. In semi-infite systems one can however have solutions in the form of evanescent states that originate from the edge of the system. One can find the energies of these edge states from the edge Green's functions, Eqs. 5.18 and 5.19, which have isolated poles at these energies. Obviously in numerical calculations one has to work at complex energies $E+i \eta$ to avoid these poles, but $\eta$ can be chosen small.

Alternatively, edge states can be obtained from the solutions of the eigenvalue problem, Eq. 5.8, solved at real energies $E$ [195, 208, 211, 212]. As edge states should decay away from the edge, only the $\mathbf{u}_{n}^{-}$modes can contribute to an edge state for a left semi-infinite system, and only the $\mathbf{u}_{n}^{+}$modes for a right semi-infinite system. An edge state of a left semi-infinite system has amplitude zero beyond the edge of that system, i.e., $\mathbf{c}_{1}=\mathbf{F}^{-} \mathbf{c}_{0}^{-}=0$ (Eq. 5.14). This means $\operatorname{rank}\left(\mathbf{F}^{-}\right)<N^{-}$, the number of left-going solutions of Eq. 5.8. Because $\operatorname{rank}\left(\mathbf{F}^{-}\right)=\operatorname{rank}\left(\mathbf{U}^{-}\right)$, see Eq. 5.12 with $\operatorname{rank}\left(\Lambda^{-}\right)=N^{-}$and $\operatorname{rank}\left(\mathbf{U}^{-}\right)=\operatorname{rank}\left(\widetilde{\mathbf{U}}^{-}\right)$, a necessary and sufficient condition for the existence of an edge state is that the eigenmodes $\mathbf{u}_{n}^{-}$are linearly dependent. A similar reasoning holds for the edge states of a right semi-infinite system. The number of edge states at a particular energy $E$ and wave number $k$ of a left/right semi-infinite system is then given by $N_{e}^{L / R}=N^{-/+}-\operatorname{rank}\left(\mathbf{U}^{-/+}\right)$.

\section{Ideal grain boundaries}

A model for an ideal grain boundary is shown in Fig. 5.2. Space is divided into two parts with $\mathbf{B}^{\prime}$ the hopping matrix block connecting the left and right halves. We assume that the on-strip Hamiltonian matrix blocks of all the strips in the left half are given by $\mathbf{H}_{L}$ right up to the boundary, and that the hopping matrix blocks between all nearest neighbor strips in the left part are given by $\mathbf{B}_{L}$. The corresponding matrix blocks for the right half are $\mathbf{H}_{R}$ and $\mathbf{B}_{R}$, respectively. The Green's function matrix blocks $\mathbf{g}_{L}^{I}$ and $\mathbf{g}_{R}^{I}$ pertaining to the two strips just left and right of the grain boundary interface can be derived like Eq. 5.6

$$
\begin{aligned}
& \mathbf{g}_{L}^{I}=\left[\mathbf{g}_{L}^{-1}-\mathbf{B}^{\prime \dagger} \mathbf{g}_{R} \mathbf{B}^{\prime}\right]^{-1} \\
& \mathbf{g}_{R}^{I}=\left[\mathbf{g}_{R}^{-1}-\mathbf{B}^{\prime} \mathbf{g}_{L} \mathbf{B}^{\prime \dagger}\right]^{-1},
\end{aligned}
$$

where $\mathbf{g}_{L}$ and $\mathbf{g}_{R}$ are the edge Green functions of the left and right semi-infinite systems, respectively. With Eqs. 5.18 and 5.19 one can express the interface Green's 


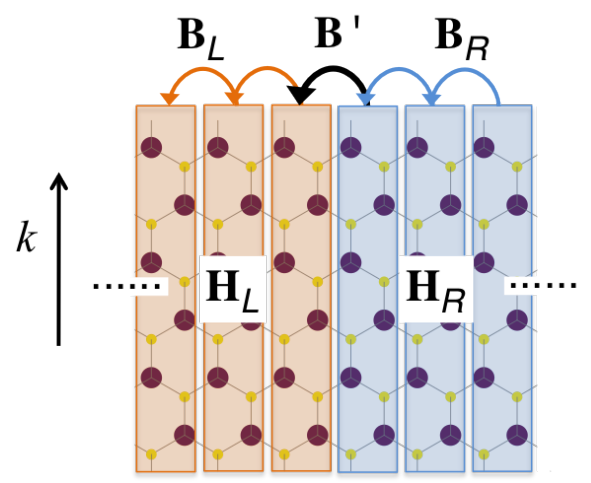

Figure 5.2: (Color online) Grain boundary between a left semi-infinite system with on-strip Hamiltonian matrix blocks $\mathbf{H}_{L}(k)$ and hopping matrix blocks $\mathbf{B}_{L}(k)$ and a right semi-infinite system with matrix blocks $\mathbf{H}_{R}(k)$ and $\mathbf{B}_{R}(k)$. The coupling between left and right parts is given by the hopping matrix block $\mathbf{B}^{\prime}(k)$.

functions in terms of Bloch matrices

$$
\begin{aligned}
& \mathbf{g}_{L}^{I}=\left[\mathbf{B}_{L}^{\dagger} \mathbf{F}_{L}^{-}-\mathbf{B}^{\prime}\left(\mathbf{B}_{R}\left(\mathbf{F}_{R}^{+}\right)^{-1}\right)^{-1} \mathbf{B}^{\prime \dagger}\right]^{-1}, \\
& \mathbf{g}_{R}^{I}=\left[\mathbf{B}_{R}\left(\mathbf{F}_{R}^{+}\right)^{-1}-\mathbf{B}^{\prime}\left(\mathbf{B}_{L} \mathbf{F}_{L}^{-}\right)^{-1} \mathbf{B}^{\prime \dagger}\right]^{-1} .
\end{aligned}
$$

\section{Modified edges}

So far we have assumed that all layers are identical right up to an edge or grain boundary. The formation of an edge or boundary often involves an electronic and a structural reconstruction, which makes the Hamiltonian matrix blocks of the strips adjacent to an edge or grain boundary different from those of the bulk strips.

We illustrate this on a left semi-infinite system with an edge layer $(i=0)$ that is different from the bulk. The tight-binding equations for the two layers closest to the edge are

$$
\begin{aligned}
-\mathbf{B} \mathbf{c}_{-2}+(E \mathbf{I}-\mathbf{H}) \mathbf{c}_{-1}-\mathbf{B}^{\prime \dagger} \mathbf{c}_{0}= & 0 \\
-\mathbf{B}^{\prime} \mathbf{c}_{-1}+\left(E \mathbf{I}-\mathbf{H}^{\prime}\right) \mathbf{c}_{0} & =0,
\end{aligned}
$$

where $\mathbf{H}^{\prime} \neq \mathbf{H}$ is the on-site Hamiltonian of the edge layer, and $\mathbf{B}^{\prime} \neq \mathbf{B}$ is the coupling of this layer to the rest of the system, see Fig. 5.3. Using $\mathbf{c}_{-2}=\left(\mathbf{F}^{-}\right)^{-1} \mathbf{c}_{-1}$, see Eqs. 5.14 and 5.15, transforms Eq. 5.25 into

$$
\left(\begin{array}{cc}
E \mathbf{I}-\mathbf{H}-\mathbf{B}\left(\mathbf{F}^{-}\right)^{-1} & -\mathbf{B}^{\prime \dagger} \\
-\mathbf{B}^{\prime} & E \mathbf{I}-\mathbf{H}^{\prime}
\end{array}\right)\left(\begin{array}{c}
\mathbf{c}_{-1} \\
\mathbf{c}_{0}
\end{array}\right)=\left(\begin{array}{l}
\mathbf{0} \\
\mathbf{0}
\end{array}\right) .
$$




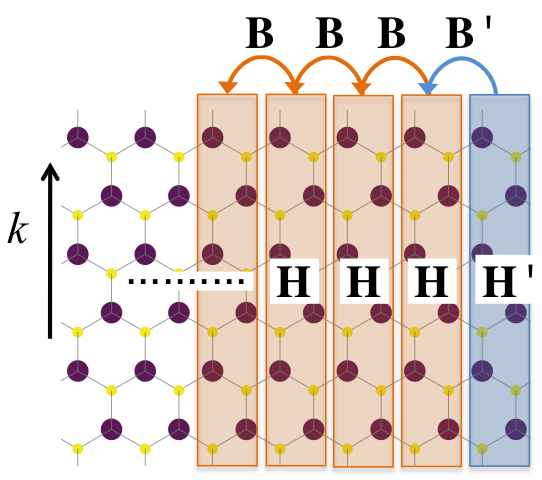

Figure 5.3: (Color online) Left semi-infinite system with a modified edge. $\mathbf{H}(k)$ and $\mathbf{B}(k)$ are the matrix blocks of the unperturbed system; $\mathbf{H}^{\prime}(k)$ and $\mathbf{B}^{\prime}(k)$ are the matrix blocks of the perturbed edge strip.

In the terminology used in non-equilibrium Green's function (NEGF) transport calculations, the term $\boldsymbol{\Sigma}_{L}(E) \equiv \mathbf{B}\left(\mathbf{F}^{-}\right)^{-1}$ is called the self-energy of the left lead [200]. The Green's function matrix blocks pertaining to the two layers closest to the edge are then given by

$$
\mathbf{G}_{L}=\left(\begin{array}{cc}
E \mathbf{I}-\mathbf{H}-\mathbf{B}\left(\mathbf{F}^{-}\right)^{-1} & -\mathbf{B}^{\prime \dagger} \\
-\mathbf{B}^{\prime} & E \mathbf{I}-\mathbf{H}^{\prime}
\end{array}\right)^{-1}
$$

The Green's function gives the density of states in the usual way, cf. Eq. 5.5. The density of states is zero for energies inside the band gap, except for isolated poles at particular energies, which represent the edge states of the modified edges. This formalism can be adapted in an obvious way to model modified edges of right semiinfinite systems, or grain boundaries where the strips left and right of the interface are modified.

\section{Charge neutrality level}

Experimentally the Fermi level in $\mathrm{MX}_{2}$ compounds is often determined by unintentional doping due to defects [146, 213, 214]. In addition, as $\mathrm{MX}_{2}$ compounds are polar materials, an internal electric field is created if a crystallite is terminated by polar edges [41, 215]. Such an electric field can cause a long range charge transfer between different edges, even if the bulk material does not contain any impurities. The position of the Fermi level can then become dependent on the size and the shape of the sample, which makes the intrinsic Fermi level an ill-defined quantity.

Each edge or grain boundary has a well-defined energy level at which that edge or grain boundary is electrically neutral, the charge neutrality level (CNL). We use the CNL as a reference point in the following. The charge neutrality level $E_{\mathrm{CNL}}$ is 
defined as the energy at which

$$
N\left(E_{\mathrm{CNL}}\right)=N_{\text {strip }}
$$

where $N_{\text {strip }}$ is the number of electrons that makes the strip neutral, and $N(E)$ is the electron counting function

$$
N(E)=\int_{-\infty}^{E} n\left(E^{\prime}\right) d E^{\prime}
$$

with $n(E)$ is the $k$-integrated density of states

$$
n(E)=\frac{a}{2 \pi} \int_{-\frac{\pi}{a}}^{\frac{\pi}{a}} n(E, k) d k .
$$

The $k$-resolved density of states $n(E, k)$ can be obtained from Eq. 5.5 for edge strips, and a similar expression for a bulk strip.

\subsection{2 $\mathrm{MX}_{2}$ edges}

The hexagonal lattice of $\mathrm{MX}_{2}$ is shown in Fig. 5.4. We specify an edge starting from a supercell spanned by vectors $\mathbf{T}_{1}$ and $\mathbf{T}_{2}$. This supercell is used to define a semiinfinite system, choosing one of the vectors as the translation vector parallel to the edge.

\section{Zigzag and armchair edges}

Similar to graphene the basic-type edges of the $\mathrm{MX}_{2}$ lattice are the zigzag and armchair edges as defined in Fig. 5.4. A zigzag edge is defined by $\mathbf{T}_{1}=\mathbf{a}_{1}$ and $\mathbf{T}_{2}=\mathbf{a}_{2}$, with $\mathbf{T}_{2}$ as the vector parallel to the edge. The matrix blocks discussed in section 5.2.1 become

$$
\begin{aligned}
\mathbf{H} & =\mathbf{H}_{0,0}+\mathbf{H}_{0,1} e^{i 2 \pi k}+\mathbf{H}_{0-1} e^{-i 2 \pi k}, \\
\mathbf{B} & =\mathbf{H}_{-1,0}+\mathbf{H}_{-1,-1} e^{-i 2 \pi k},
\end{aligned}
$$

where $\mathbf{H}_{p, q}$ denotes the real space Hamiltonian matrix block that describes the interaction between atoms in the unit cell situated at the origin and atoms in the unit cell situated at $p \mathbf{a}_{1}+q \mathbf{a}_{2}$. The matrix elements of $\mathbf{H}_{p, q}$ depend on the specific tightbinding model that is used to represent the electronic structure of $\mathrm{MX}_{2}$. They are given in the appendices.

Note that by solving the quadratic eigenvalue equation, Eqs. 5.8 and 5.9, one has simultaneous access to the Green's functions of both the edges of a right and a left semi-infinite system via Eqs. 5.18 and 5.19. Unlike graphene the $\mathrm{MX}_{2}$ monolayer lacks inversion symmetry, which means that the zigzag edge termination of a right semi-infinite system is different from that of a left semi-infinite system, see Fig. 5.4. 


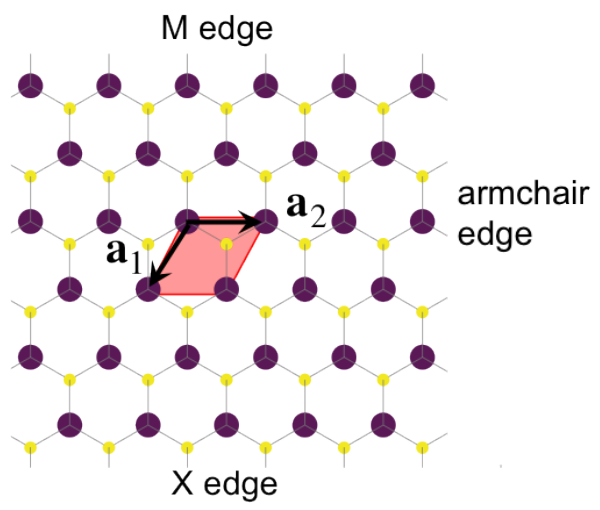

Figure 5.4: (Color online) Top view of the $\mathrm{MX}_{2}$ lattice with $\mathrm{M}$ and $\mathrm{X}$ atoms in purple and yellow, respectively. The lattice vectors $\mathbf{a}_{1}$ and $\mathbf{a}_{2}$ are indicated by arrows. The $M$ and $X$ zigzag edges runs parallel to $\mathbf{a}_{2}$ and are the edges of right and left semiinfinite systems, respectively. The armchair edge runs parallel to $2 \mathbf{a}_{1}+\mathbf{a}_{2}$.

The zigzag edge of a right semi-infinite system is terminated by metal atoms. We call this the M-edge, consistent with previous studies on $\mathrm{MoS}_{2}$, where it is called the Mo-edge $[174,175,176]$. The zigzag edge of a left semi-infinite system is then called the X-edge, as it is terminated by chalcogen atoms. In previous studies on $\mathrm{MoS}_{2}$, it has been called the S-edge.

An edge in armchair orientationcan be constructed from $\mathbf{T}_{1}=2 \mathbf{a}_{1}+\mathbf{a}_{2}$ and $\mathbf{T}_{2}=$ $\mathbf{a}_{2}$ with $\mathbf{T}_{1}$ the translation vector parallel to the edge, see Fig. 5.4. The corresponding supercell is twice as large as the unit cell, so the dimension of the matrix blocks defining the edge are twice the dimension of the blocks defining the zigzag edge.

$$
\begin{gathered}
\mathbf{H}=\left(\begin{array}{cc}
\mathbf{H}_{0,0} & \mathbf{H}_{1,1}+\mathbf{H}_{-1,0} e^{-i 2 \pi k} \\
\mathbf{H}_{1,0} e^{i 2 \pi k}+\mathbf{H}_{-1,-1} & \mathbf{H}_{0,0}
\end{array}\right), \\
\mathbf{B}=\left(\begin{array}{cc}
\mathbf{H}_{0,-1} & \mathbf{H}_{1,0}+\mathbf{H}_{-1,-1} e^{-i 2 \pi k} \\
0 & \mathbf{H}_{0,-1}
\end{array}\right) .
\end{gathered}
$$

Note that the termination at the edge is controlled by the contents of the cell used to define the primitive vectors $\mathbf{a}_{1}, \mathbf{a}_{2}$. In particular, the cell defined in Fig. 5.4 does not lead to a pristine armchair edge, but one with additional $\mathrm{M}$ or $\mathrm{X}$ atoms attached to the edge. It is straightforward to remove these atoms and by applying the technique outlined in Sec. 5.2.1 obtain the electronic structure of a pristine armchair edge. 


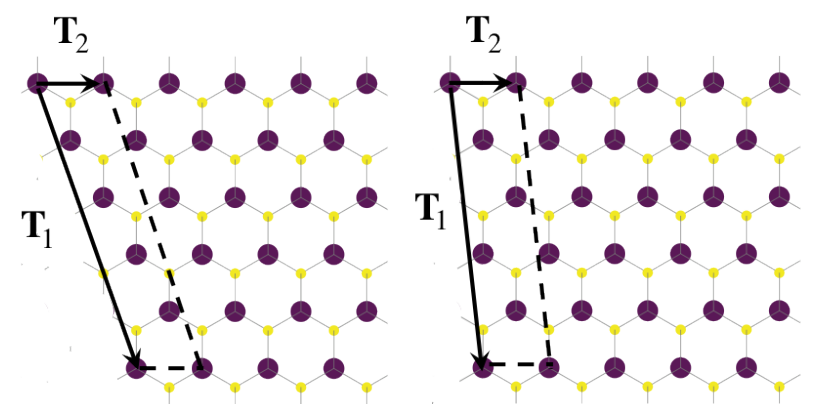

Figure 5.5: (Color online) Left: generalized zigzag edge parallel to $\mathbf{T}_{1}=3\left(\mathbf{a}_{1}+\mathbf{a}_{2}\right)+$ $\left(2 \mathbf{a}_{1}+\mathbf{a}_{2}\right)$. Right: generalized armchair edge parallel to $\mathbf{T}_{1}=\left(\mathbf{a}_{1}+\mathbf{a}_{2}\right)+2\left(2 \mathbf{a}_{1}+\mathbf{a}_{2}\right)$.

\section{General edges}

Edges with a somewhat more general orientation are defined by the supercell

$$
\mathbf{T}_{1}=m\left(\mathbf{a}_{1}+\mathbf{a}_{2}\right)+n\left(2 \mathbf{a}_{1}+\mathbf{a}_{2}\right) ; \mathbf{T}_{2}=\mathbf{a}_{2},
$$

with $\mathbf{T}_{1}$ the translation vector parallel to the edge. It is defined as $m$ zigzag vectors plus $n$ armchair vectors, see Fig. 5.5. The angle with the direction of $\mathbf{a}_{2}$ is given by

$$
\theta=\arccos \left(\frac{\frac{1}{2} m}{\sqrt{m^{2}+3 m n+3 n^{2}}}\right) .
$$

Because of the symmetry of the lattice one only has to cover the $30^{\circ}$ angle between a zigzag orientation $m=1, n=0, \theta=60^{\circ}$, and an armchair orientation $m=0, n=1$, $\theta=90^{\circ}$. Left and right edges (M-type and X-type edges) are then obtained by using $\mathbf{T}_{2}=\mathbf{a}_{2}$ and $\mathbf{T}_{2}=-\mathbf{a}_{2}$, respectively.

A series of edge orientations is obtained by setting $n=1$ and varying $m$, where the translation vector along the edge is the sum of $m$ zigzag vectors and one armchair vector. We call this a generalized zigzag edge, see Fig. 5.5. The supercell defined by the lattice vectors, Eq. 5.35, contains $m+2$ unit cells. For instance, values $m=$ 1,2 give angles $\theta=79.1^{\circ}, 70.9^{\circ}$, respectively. The construction of the Hamiltonian matrix blocks defining the edge is straightforward. As an example, the Hamiltonian matrix blocks for $m=2, n=1$ are

$$
\mathbf{H}=\left(\begin{array}{cccc}
\mathbf{H}_{0,0} & \mathbf{H}_{1,1} & 0 & \mathbf{H}_{-1,0} e^{-i 2 \pi k} \\
\mathbf{H}_{-1,-1} & \mathbf{H}_{0,0} & \mathbf{H}_{1,1} & 0 \\
0 & \mathbf{H}_{-1,-1} & \mathbf{H}_{0,0} & \mathbf{H}_{1,1} \\
\mathbf{H}_{1,0} e^{i 2 \pi k} & 0 & \mathbf{H}_{-1,-1} & \mathbf{H}_{0,0}
\end{array}\right),
$$




$$
\mathbf{B}=\left(\begin{array}{cccc}
\mathbf{H}_{0-1} & \mathbf{H}_{1,0} & 0 & \mathbf{H}_{-1,-1} e^{-i 2 \pi k} \\
0 & \mathbf{H}_{0,-1} & \mathbf{H}_{1,0} & 0 \\
0 & 0 & \mathbf{H}_{0,-1} & \mathbf{H}_{1,0} \\
0 & 0 & 0 & \mathbf{H}_{0,-1}
\end{array}\right)
$$

To generate edges with an orientation closer to the armchair edge $\left(\theta=90^{\circ}\right)$ one can use the series with $m=1$ and vary $n$. The translation vector parallel to the edge is then the sum of one zigzag vector and $n$ armchair vectors, which we call a generalized armchair edge, see Fig. 5.5. The supercell then contains $2 n+1$ unit cells. As an example, $m=1, n=3$ gives $\theta=85.3^{\circ}$. The edge termination can be controlled by adding and removing atoms at the edge, and apply the technique outlined in Sec. 5.2.1.

\subsubsection{Tight-binding models}

We consider tight-binding models with spin degeneracy, i.e., we neglect spin-orbit coupling. The main contributions to the valence and conduction bands of $\mathrm{MX}_{2}$ around the band gap come from the valence $d$-shell of the $\mathrm{M}$ atom and the $p$-shell of the $\mathrm{X}$ atoms. A minimal basis set then comprises eleven orbitals: five metal $d$ orbitals, and six $p$-orbitals of the two chalcogen atoms [104]. Monolayer $\mathrm{MX}_{2}$ has $D_{3 h}$ point-group symmetry. The trigonal prismatic coordination of the metal atom splits the $d$-states into three groups: $A_{1}^{\prime}\left\{d_{3 z^{2}-r^{2}}\right\}, E^{\prime}\left\{d_{x y}, d_{x^{2}-y^{2}}\right\}$, and $E^{\prime \prime}\left\{d_{x z}, d_{y z}\right\}$. The six $p$-orbitals of the two chalcogen atoms split into the groups $A_{1}^{\prime}\left\{p_{z}^{-}=p_{z}\left(\mathrm{X}_{1}\right)-\right.$ $\left.p_{z}\left(\mathrm{X}_{2}\right)\right\}, E^{\prime}\left\{p_{x}^{+}=p_{x}\left(\mathrm{X}_{1}\right)+p_{x}\left(\mathrm{X}_{2}\right), p_{y}^{+}=p_{y}\left(\mathrm{X}_{1}\right)+p_{y}\left(\mathrm{X}_{2}\right)\right\}, A_{2}^{\prime \prime}\left\{p_{z}^{+}=p_{z}\left(\mathrm{X}_{1}\right)+p_{z}\left(\mathrm{X}_{2}\right)\right\}$ and $E^{\prime \prime}\left\{p_{x}^{-}=p_{x}\left(\mathrm{X}_{1}\right)-p_{x}\left(\mathrm{X}_{2}\right), p_{y}^{-}=p_{y}\left(\mathrm{X}_{1}\right)-p_{y}\left(\mathrm{X}_{2}\right)\right\}$. Mirror symmetry in the plane of the metal atoms, $\sigma_{h}$, allows for hybridization between orbitals that are even with respect to $\sigma_{h}$, i.e., $A_{1}^{\prime}$ and $E^{\prime}$ orbitals, or between orbitals that are odd, i.e., $A_{2}^{\prime \prime}$ and $E^{\prime \prime}$ orbitals.

The set of orbitals with even symmetry thus comprises the six orbitals $d_{3 z^{2}-r^{2}}$, $d_{x y}, d_{x^{2}-y^{2}}, p_{z}^{-}, p_{x}^{+}$, and $p_{y}^{+}$, and the set with odd symmetry the five orbitals $d_{x z}, d_{y z}$, $p_{z}^{+}, p_{x}^{-}$, and $p_{y}^{-}$. As the even/odd symmetry is conserved for the edges and grain boundaries considered in this paper, all corresponding Hamiltonian matrices are blocked, and the even/odd solutions can be obtained separately. The matrix blocks $\mathbf{H}_{p, q}$ required for constructing the Hamiltonian matrices of Sec. 5.2.2, are given in appendix A.2. The values of the tight-binding parameters have been obtained by fitting the bulk band structure to bands obtained from density functional theory (DFT) calculations with the generalized gradient approximation (GGA/PBE) functional [59]. For the even states we use the parameters given by Rostami et al.,[205] and for the odd states we use the parameters given in appendix A.2.

The eleven band model can be simplified further. From early theoretical studies and recent first-principles calculations one observes that the $\mathrm{MX}_{2}$ bands at the top of the valence band and at the bottom of the conduction band, are dominated by the metal $d$-orbitals, in particular those with even symmetry, i.e., $d_{3 z^{2}-r^{2}}, d_{x y}$, and $d_{x^{2}-y^{2}}$ $[151,216,104]$. Contributions to the bands around the gap from the $d_{x z}$ and $d_{y z}$ or- 

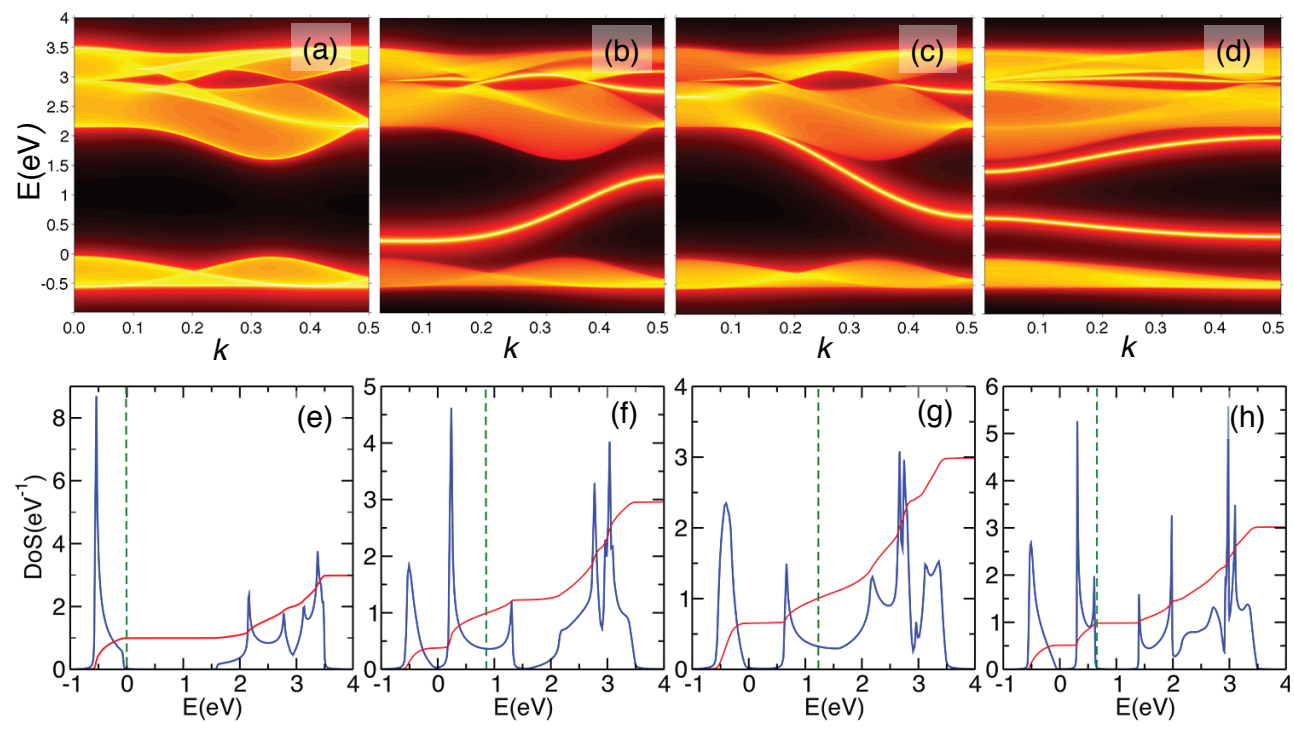

Figure 5.6: (Color online) (a) $k$-resolved density of states (DoS) per $\mathrm{MoS}_{2}$ unit of bulk $\mathrm{MoS}_{2}$ in the three band tight-binding model, with the $k$-vector parallel to the zigzag edge, and the zero of energy at the top of the valence band. The DoS plotted is on a logarithmic scale (right-hand side $n$ denotes amplitude $10^{-n}$ ) using a broadening parameter $\eta=0.05 \mathrm{eV}$ (Eq. 5.1). (b), (c), (d) $k$-resolved DoSs of the Mo-edge, the Sedge, and the armchair edge, respectively. (e),(f),(g),(h) $k$-integrated DoS per $\mathrm{MoS}_{2}$ unit of bulk $\mathrm{MoS}_{2}$, the Mo-edge, the S-edge, and the armchair edge, all plotted on a linear scale $(\eta=0.05 \mathrm{eV})$. The red solid lines give the counting function and the green dashed lines indicate the charge neutrality level (CNL), Eqs. 5.28-5.30.

bitals and from the chalcogen $p$-orbitals are much smaller. Matheiss has constructed an effective tight-binding model for $\mathrm{MX}_{2}$, where only the metal sites are taken into account explicitly [151]. These sites form a two-dimensional triangular lattice, and the presence of the $\mathrm{X}$ atoms lowers the symmetry of this lattice from $D_{6 h}$ to $D_{3 h}$. Only the metal orbitals with even symmetry, $d_{3 z^{2}-r^{2}}, d_{x y}$, and $d_{x^{2}-y^{2}}$, are used to construct an effective three-band model. The matrix blocks $\mathbf{H}_{p, q}$ of this model are given in appendix A.1. We use the parameters given by Liu et al.,[204], which have been obtained by fitting the bulk bands to GGA/PBE results. 


\subsection{Results}

\subsubsection{Three-band model}

\section{Zigzag and armchair edges}

The eigenvalues and eigenvectors of Eq. 5.8, are used to construct the Bloch matrices, Eq. 5.12. The bulk density of states $n(k, E)$ is shown in Fig. 5.6(a), as calculated from the Green's function matrix of a bulk strip, Eqs. 5.2 and 5.20, in the zigzag edge orientation. In the three-band model, the lowest band is the valence band, and the overlapping two upper bands form the conduction band. Note that the zero of energy is chosen at the top of the valence band. The same Bloch matrices give access to the Green's function matrices of the zigzag edge strips, Eqs. 5.18 and 5.19. Here $\mathbf{g}_{R}(k, E)$ and $\mathbf{g}_{L}(k, E)$ are the energy and $k$-resolved Green's function of the M-edge and the X-edge, respectively, see Fig. 5.4. Again using $\mathrm{MoS}_{2}$ as an example, the associated densities of state $n_{R}(k, E)$ and $n_{L}(k, E)$ of the Mo-edge, respectively the S-edge, are shown in Fig. 5.6(b) and (c).

The Mo-edge has a prominent edge state inside the bulk band gap dispersing upwards from $k=0$ to $k=\frac{1}{2}$. The S-edge has an edge state that starts in the bulk conduction band at $k=0$, and disperses downward to $k=\frac{1}{2}$. In surface physics such states are termed Shockley states [217]. These edge states are also found in the more detailed eleven-band model with a similar dispersion, see Sec. 5.3.2. The latter model shows a richer edge state structure, originating from bands that are omitted in the simple three-band model. Nevertheless, the three-band model finds prominent Mo-edge and the S-edge states with the correct dispersion. Edge states are also found at $\sim 3 \mathrm{eV}$, which is in the hybridization gap between the two conduction bands.

The structure of a bulk strip in armchair orientation has a mirror plane perpendicular to the $\mathrm{MX}_{2}$-plane and along the armchair orientation, see see Fig. 5.4. It follows that the densities of states of right and left edges, $n_{R}(k, E)$ and $n_{L}(k, E)$, are identical for the armchair orientation. The $k$-resolved density of states of the $\mathrm{MoS}_{2}$ armchair edge, Eq. 5.33-5.34, is shown in Fig. 5.6(d). There are two clear edge states with energies in the band gap. One edge state is just below the conduction band and roughly follows the dispersion of the conduction band edge, whereas the other one is positioned at $\sim 0.5 \mathrm{eV}$ above the valence band, following the dispersion of valence band edge.

The $k$-integrated densities of states, Eq. 5.30, of a bulk $\mathrm{MoS}_{2}$ strip in zigzag orientation, the Mo-edge, the S-edge, and the armchair edge, are given in Figs. 5.6(e)-(h), respectively. These densities of states show the van Hove singularities at the band edges that are typical of 1D structures. With the zero of energy at the top of the valence band, the bulk valence band lies in the energy range $-0.5-0.0 \mathrm{eV}$, and the two conduction bands in the range 1.6-3.5 eV, see Fig. 5.6(e). The Mo-edge shows edge bands in the energy range $0.1-1.4 \mathrm{eV}$, and around $3 \mathrm{eV}$. The S-edge has an edge band starting at $0.5 \mathrm{eV}$, which merges with the conduction band at higher energies, and additional edge states with energies 2.7-3.0 eV. The armchair edge has two edge 
band in the gap at in the energy ranges $0.3-0.6 \mathrm{eV}$ and $1.4-2.0 \mathrm{eV}$, respectively, and an edge state around $3.1 \mathrm{eV}$.

Also shown in Figs. 5.6(e)-(h) are the electron counting function (red curves), Eq. 5.29, and the charge neutrality level (CNL; green lines), Eq. 5.28. Obviously for the bulk the CNL is at the top of the valence band, see Fig. 5.6(e). The position of the CNL at the Mo-edge corresponds to a 2/3 filled edge band in the gap, see Fig. 5.6(f). One might interpret this as effectively two-third of the bonds being broken for a Mo atom at the Mo-edge. Likewise, the position of the CNL at the S-edge corresponds to a 1/3 filled edge band in the gap, see Fig. 5.6(g). This correlates with one-third of the bonds being broken for a Mo atom at the S-edge. The supercell of the armchair edge has two Mo atoms along the edge, where the local environments of one is similar to that of a Mo atom at an Mo-edge, and the local environment of the other is similar to that of a Mo atom at the S-edge, see Fig. 5.4. Summing the $2 / 3$ and $1 / 3$ edge state occupations at the Mo- and S-edges, one predicts that the armchair edge has one completely filled edge band. The calculated CNL shows that this is indeed the case; the armchair edge has one fully occupied edge band, and one empty edge band, see see Fig. 5.6(h).

The CNLs of all three basic edges are quite close, cf. Figs. 5.6(f)-(h). This is an artefact of the three-band model, and of disregarding the odd bands in particular. The latter play an important role in setting the CNLs in $\mathrm{MX}_{2}$ edges, as we will discuss in Sec 5.3.2. In polar lattices such as $\mathrm{MX}_{2}$ the CNL does not fix the intrinsic Fermi level, unlike in a non-polar lattice such as graphene $[41,215]$. In a finite-sized $\mathrm{MX}_{2}$ sample electrons can be redistributed among all the edges in the sample, driven by the internal electric field set up by the intrinsic polarization of the material.

So far we have focused on edges of $\mathrm{MoS}_{2}$, but all $\mathrm{MX}_{2}$ compounds with a similar structure ( $D_{3 h}$ point group, trigonal prismatic coordination of $\mathrm{M}$ atoms by $\mathrm{X}$ atoms) have similar edge structures. Figure 5.7 shows the densities of states of the $\mathrm{W}$ and $\mathrm{Te}$ zigzag edges of $H-\mathrm{WTe}_{2}$ as an example. These densities of states are very similar to those of the corresponding Mo- and S-edges of $\mathrm{MoS}_{2}$, see Figs. 5.6(b), (c), (f) and (g). The band gap of bulk WTe 2 is somewhat smaller than that of $\mathrm{MoS}_{2}$, and the band widths are somewhat larger. $\mathrm{WTe}_{2}$ has a band gap of $1.1 \mathrm{eV}$ (GGA/PBE), a valence band in the range $-0.7-0.0 \mathrm{eV}$, and conduction bands in the range 1.1-3.7 eV. The $\mathrm{W}$ edge has a state in the gap in the energy range $0.0-1.4 \mathrm{eV}$, and the Te-edge has a state in the gap in the range $0.5-2.0 \mathrm{eV}$. As is the case for the bulk bands, the band widths of these edge states are somewhat larger than the corresponding states in $\mathrm{MoS}_{2}$.

\section{General edges and grain boundaries}

The electronic structure of the more general edges defined in Sec. 5.2.2 can be calculated along the same lines. Figs. 5.8(a) and (b) give the densities of states of generalized zigzag edges of $\mathrm{MoS}_{2}$ with translation vectors along the edge defined by $m=1$ and 3, respectively, and $n=1$, see Eq. 5.35 and Fig. 5.5. The generalized zigzag edge has a rich structure of edge states within the bulk band gap. The peak 

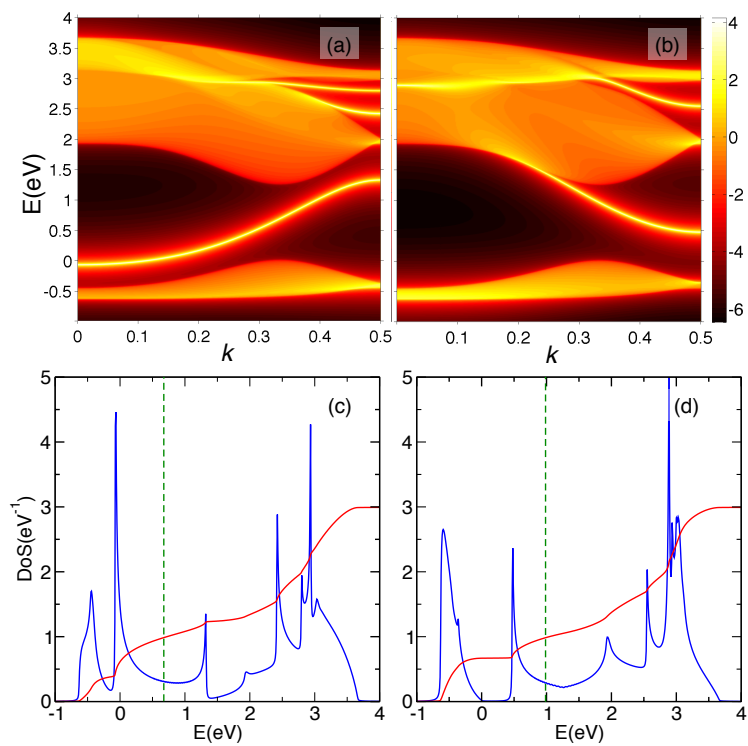

Figure 5.7: (Color online) (a),(b) $k$-resolved DoSs of the W-edge and the Te-edge within the three band tight-binding model of $H$-WTe 2 . (c),(d) $k$-integrated DoSs of the $\mathrm{W}$-edge and the Te-edge. The same conventions and parameters are used as in Fig. 5.6.

at $0.4 \mathrm{eV}$ in Figs. 5.8(a) and (b) results from the armchair part of this edge, compare to Fig. 5.6(h). The band in the range $1-1.5 \mathrm{eV}$ in $5.8(\mathrm{a})$, and the structure of bands in the range $0.8-1.9 \mathrm{eV}$ in 5.8(b) originates mainly from the zigzag parts.

The counting system for the three-band model, as outlined in the previous section, gives a filling 2/3 per Mo atom at a Mo-edge and a filling 1 per two Mo atoms at an armchair edge. For a generalized zigzag edge one would then predict the CNL to correspond to $2 m / 3+n$ filled edge states. This would mean that the CNL is inside an edge band, unless $m$ is a multiple of three. This is confirmed by a calculation of the CNL according to Eq. 5.28. In Fig. 5.6(a), where $m=1$, the position of the CNL corresponds to a 2/3-filled edge band, whereas in Fig. 5.6(b), where $m=3$, the CNL is in a gap between two edge states. The gaps between the edge states of the general edges are quite small however. In a sample that involves long range charge transfer between the edges, as discussed above, these semiconducting edges easily become doped.

Figures 5.8(c)and (d) show the densities of states of generalized armchair edges of $\mathrm{MoS}_{2}$, with translation vectors along the edge with $m=1$ and $n=2$ and 3, respectively, see Eq. 5.35 and Fig. 5.5. Again this general edge has a rich structure of states within the bulk band gap. In particular the peaks around $0.4 \mathrm{eV}$ and 1.7 $\mathrm{eV}$ have a strong armchair character. The counting model gives $1 / 3+n$ filled edge states, so it predicts that the CNL always lies inside an edge band. Calculations of the 

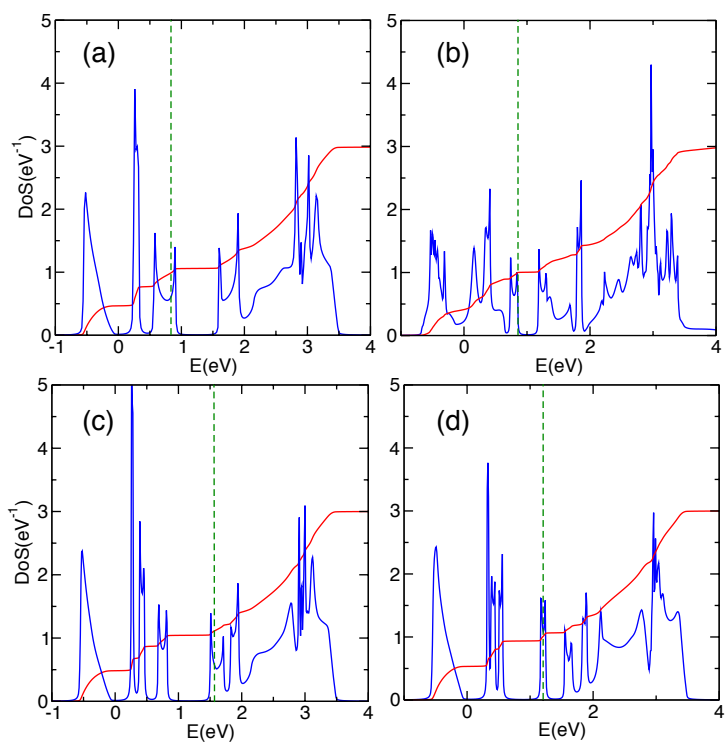

Figure 5.8: (Color online) $k$-integrated DoSs of the generalized zigzag edges defined by Eq. 5.35 with (a) $m=1, n=1$ and (b) $m=3, n=1$, and of the generalized armchair edges with (c) $m=1, n=2$ and (d) $m=1, n=3$.

CNL according to Eq. 5.28 confirm this, as shown in Fig. 5.8(c) and (d). In conclusion, within the three-band tight-binding model, pristine charge neutral edges are metallic for most edge orientations.

A similar analysis can be applied to the states found at grain boundaries. We illustrate the use of Eq. 5.21 for calculating the states at grain boundaries using a simple grain boundary in $\mathrm{MoS}_{2}$. It consists of a left semi-infinite part, terminated by a S-edge, connected to a right semi-infinite part, terminated by a Mo-edge. As hopping matrix defining the coupling between left and right parts, Fig. 5.2, we choose a scaled version of the bulk hopping matrix, Eq. 5.32.

$$
\mathbf{B}^{\prime}=\alpha \mathbf{B} ; 0 \leq \alpha \leq 1 .
$$

Obviously $\alpha=0$ gives two uncoupled S- and Mo-edges with corresponding edge states, Figs. 5.6(b), (c), (f) and (g), whereas $\alpha=1$ gives the bulk electronic structure, Figs. 5.6(a) and (e), without any edge states. The values $0<\alpha<1$ then represent a simple model for a weak link with zigzag orientation.

Figure 5.9(a) gives the $k$-resolved density of states for $\alpha=0.2$, which represents a relatively weak coupling between the left and right parts. In the band gap one observes the two edge bands that are typical of the Mo-edge and the S-edge, see Figs. 5.6(b) and (c). Due to the coupling between the S- and Mo-edges at the grain boundary, the two bands interact, which results in an avoided crossing between the 

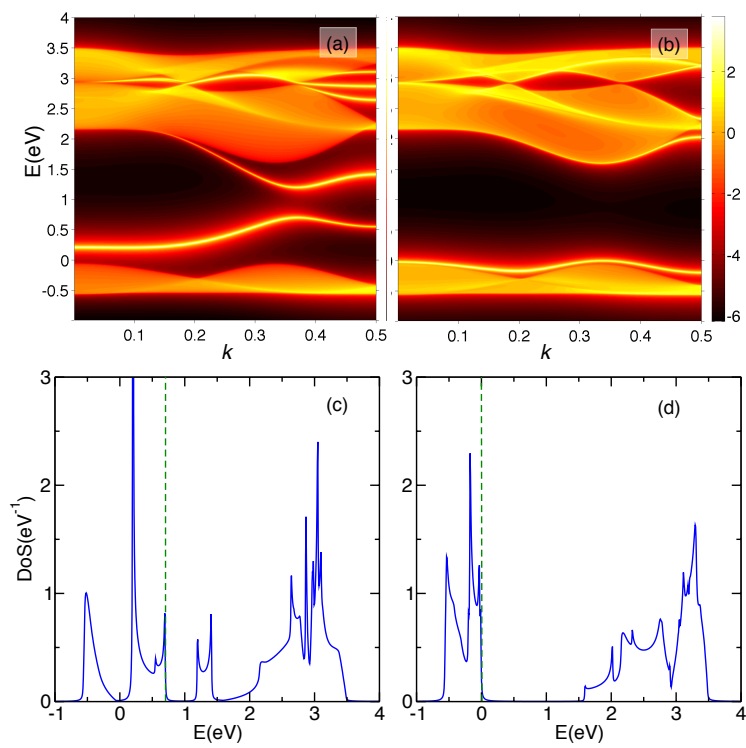

Figure 5.9: (Color online) (a) $k$-resolved DoS of a model grain boundary between a Mo-edge and a S-edge, with a coupling between the edges $\alpha=0.2$, Eq. 5.32. (b) $k$-resolved DoS with $\alpha=0.8$. (c),(d) the corresponding $k$-integrated DoSs.

two edge states at $k \approx 0.35$ and $E \approx 1 \mathrm{eV}$. The avoided crossing creates an energy gap in the range $0.7-1.2 \mathrm{eV}$, which is clearly visible in the $k$-integrated density of states for $\alpha=0.2$, shown in Fig. 5.9(c). The counting model predicts 2/3, respectively $1 / 3$ filling per Mo atom of an edge state at the Mo-edge and the S-edge, if the grain boundary is charge neutral. This implies that the lower edge band is fully occupied, whereas the upper edge band is empty. A calculation of the CNL according to Eq. 5.28 confirms this, see Fig. 5.9(c).

Figure 5.9(b) gives the $k$-resolved density of states for a strong coupling between the left and right parts, $\alpha=0.8$. The interaction between the two edge bands is now much stronger than for the case discussed in the previous paragraph, which results in a much larger gap in the range 0.2-1.6 eV, see Fig. 5.9(d). The two edge bands are pushed toward the valence band and the conduction band, respectively, and they more closely follow the dispersions of the valence and conduction band edges. The occupancy of a charge neutral grain boundary is the same as before, i.e., a fully occupied lower edge band and an empty upper edge band.

As a final point in this section we illustrate the technique introduced in Sec. 5.2.1 for handling edges that involve an electronic or a structural reconstruction. For the zigzag edges we have found a $2 / 3$ respectively a $1 / 3$ occupied edge band for the Mo-edge and the S-edge. It suggests that a reconstruction that triples the translational period along the edge, may lead to fully occupied edge states for both edges. As a proof of principle, we test a very simple reconstruction, where one in three 

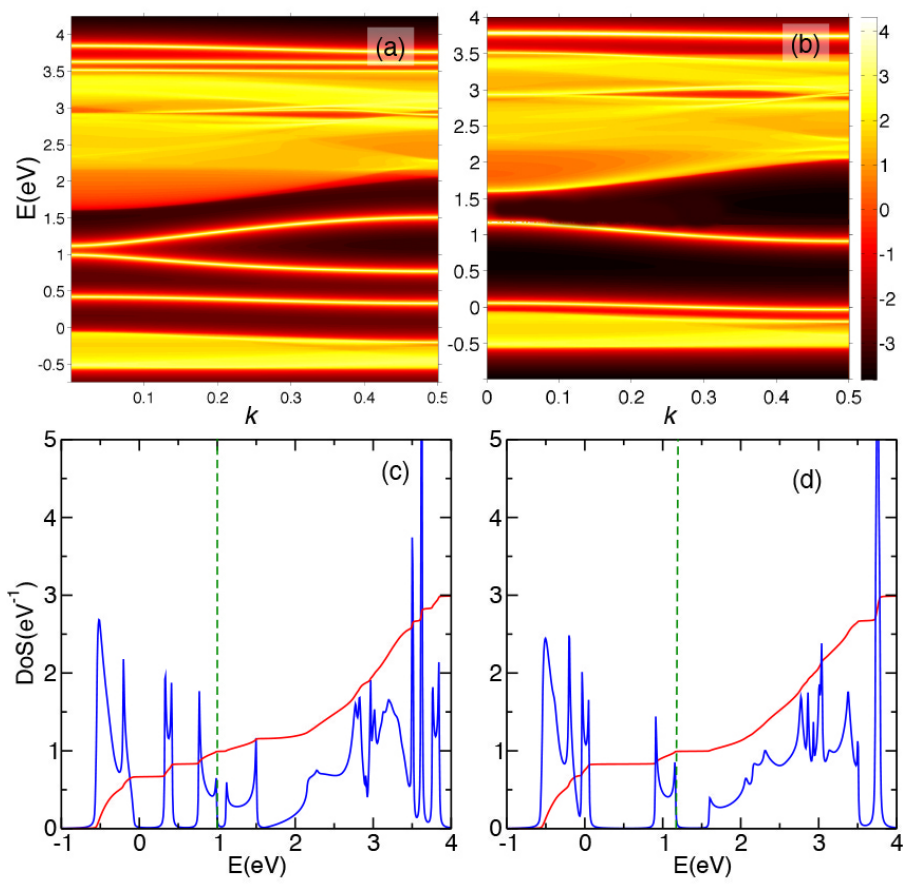

Figure 5.10: (Color online) (a,c) $k$-resolved and $k$-integrated DoS of a Mo-edge with a $3 \times$ reconstruction, where the on-site energy of every third Mo-atom is $1 \mathrm{eV}$ lower than that of the other two; $(b, d)$ the same information for the S-edge.

Mo atoms at the Mo-edge or the S-edge has a different on-site energy. The Green's function matrix is calculated from Eq. 5.25. The densities of states of the modified edge layers are shown in Fig. 5.10(a) and (c) for the Mo-edge and (b) and (d) for the S-edge. Obviously increasing the periodicity from $1 \times$ to $3 \times$ folds the edge band, so one observes three edge states instead of one. Decreasing the on-site energy of every third Mo atom at the edge by $1 \mathrm{eV}$ introduces energy gaps between the edge states.

By calculating the CNL we observe that at the Mo-edge the two lowest edge bands, starting at $0.4 \mathrm{eV}$ and $0.7 \mathrm{eV}$ in Fig. 5.10(b) and (d), are filled, whereas the third band, starting at $1.1 \mathrm{eV}$, is empty. The lowest edge band of the S-edge at $0.0 \mathrm{eV}$, Fig. 5.10(a) and (c), is filled, whereas the two upper bands, starting at $0.9 \mathrm{eV}$ and 1.6 $\mathrm{eV}$, respectively, are empty.

\subsubsection{Eleven-band tight-binding model}

As discussed in Sec. 5.2.3, the eleven-band tight-binding model of $\mathrm{MX}_{2}$ uses a basis set composed of the five valence $d$-orbitals of the $\mathrm{M}$ atom, and the six valence $p$ orbitals of the two $\mathrm{X}$ atoms. Because mirror symmetry $\sigma_{h}$ in the $\mathrm{MX}_{2}$ plane holds both for monolayers as well as for edges, states that are even or odd with respect 

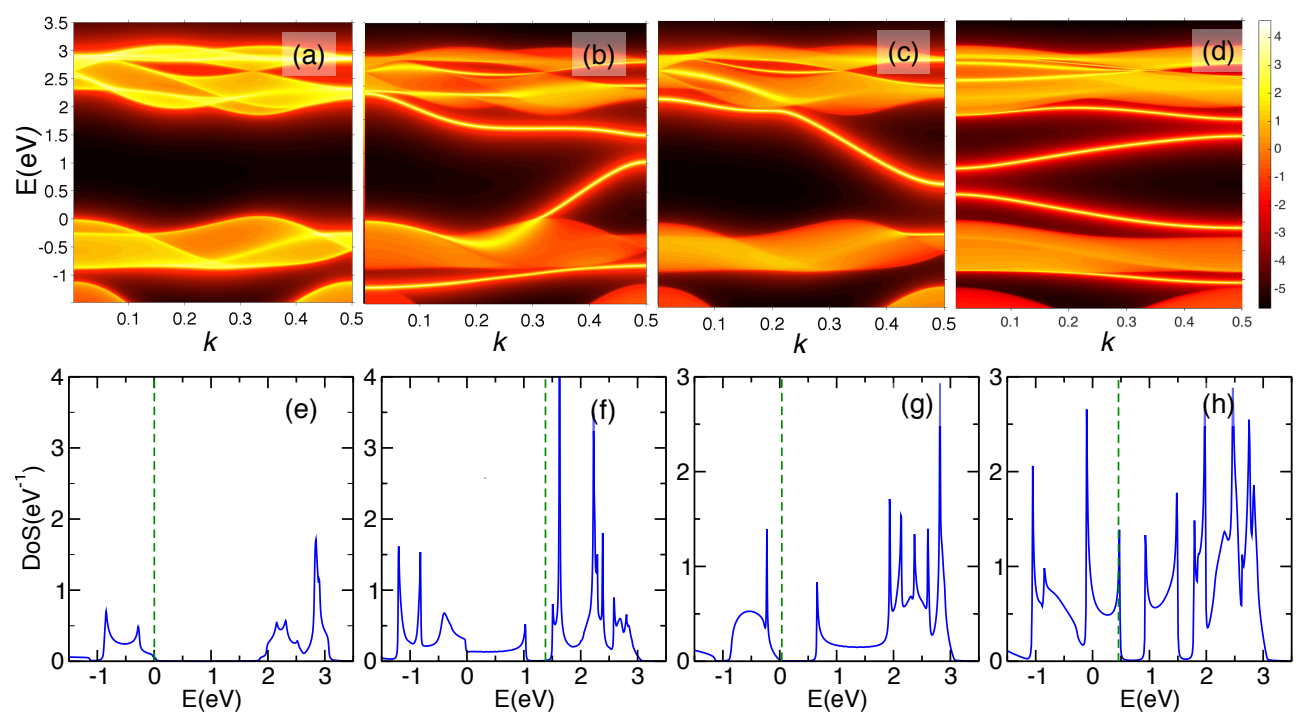

Figure 5.11: (Color online) As Fig. 5.6 but for the states within the eleven band model with even symmetry with respect to a mirror plane through the plane of the Mo atoms. (a),(b),(c),(d) $k$-resolved DoS per $\mathrm{MoS}_{2}$ unit of bulk $\mathrm{MoS}_{2}$, the Mo-edge, the S-edge, and the armchair edge, respectively. (e),(f),(g),(h) The corresponding $k$ integrated DoSs of bulk $\mathrm{MoS}_{2}$, the Mo-edge, the S-edge, and the armchair edge. The same conventions and parameters are used as in Fig. 5.6.

this mirror symmetry are treated separately. The even symmetry set comprises the six orbitals $d_{3 z^{2}-r^{2}}, d_{x y}, d_{x^{2}-y^{2}}, p_{z}^{-}, p_{x}^{+}$, and $p_{y}^{+}$, and the odd symmetry set the five orbitals $d_{x z}, d_{y z}, p_{z}^{+}, p_{x}^{-}$, and $p_{y}^{-}$, leading to six- and five-dimensional Hamiltonian matrices, respectively.

Again we use $\mathrm{MoS}_{2}$ as an example of a $\mathrm{MX}_{2}$ monolayer. The procedure for obtaining the Green's functions and the densities of states are the same as those described in the previous section. In the following we will only discuss the results obtained for the basic edges, the zigzag and the armchair edges, and compare those to the results obtained with the three-band model.

\section{Even bands}

The $k$-resolved density of states of the even states of a bulk strip in the zigzag edge orientation is shown in Fig. 5.11(a) in an energy region around the band gap, and the corresponding $k$-integrated density of states is given in Fig. 5.11(e). As before, the zero of energy is positioned at the top of the valence band. Qualitatively, these densities of states are similar to those of the three-band model, compare to Figs. 5.6(a) and (e). Quantitatively, the eleven-band model gives a highest valence band that is wider by $\sim 0.3 \mathrm{eV}$, whereas the two lowest conduction bands are narrower by $\sim 0.5$ 

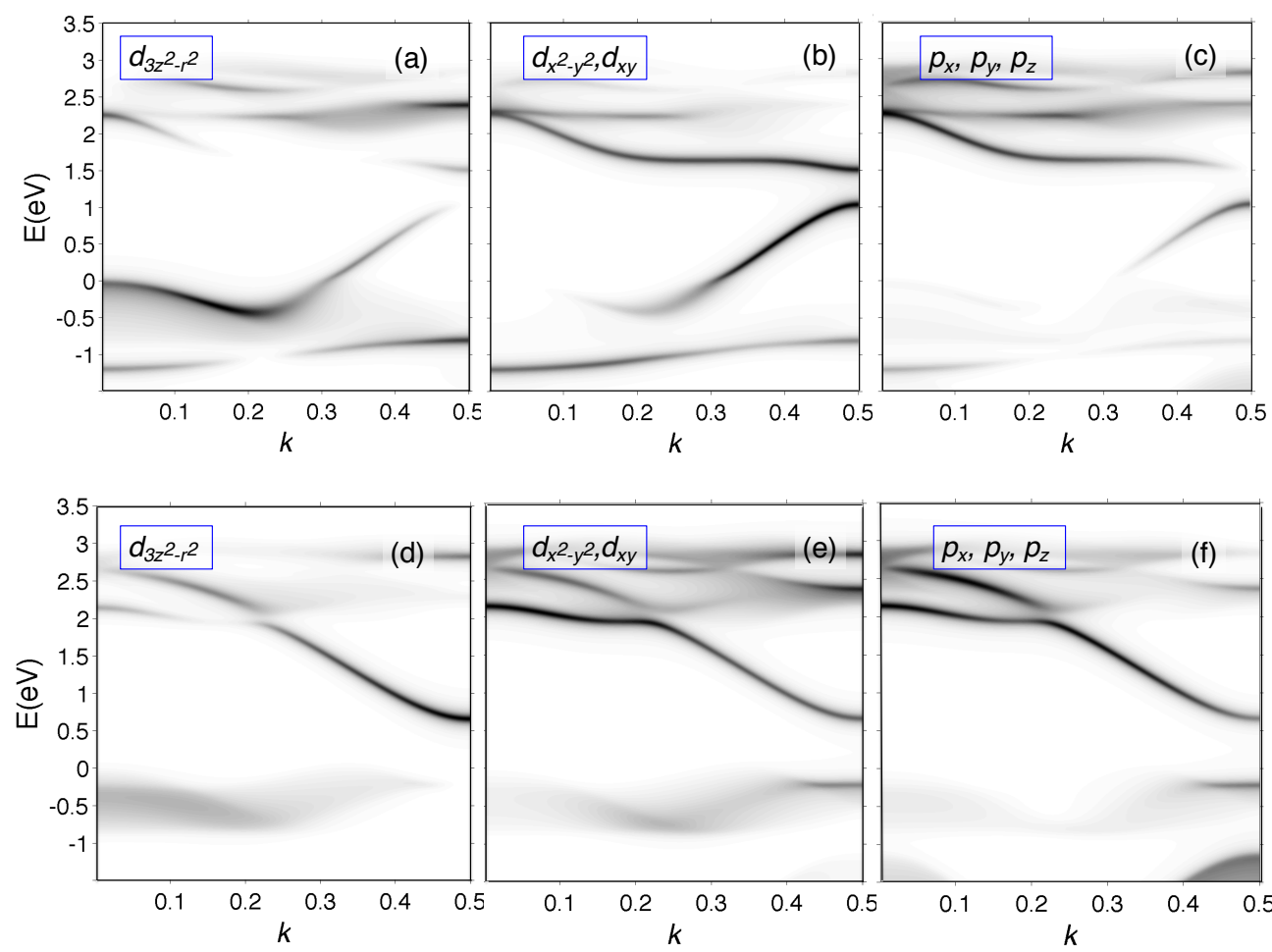

Figure 5.12: (Color online) Projected densities of states (PDoS) calculated according to Eq. 5.5. (a),(b),(c) For the Mo-edge, projected on the Mo $d_{3 z^{2}-r^{2}}$ orbital, the $d_{x y}$ and $d_{x^{2}-y^{2}}$ orbitals, and the $p$ orbitals of the S atoms, respectively. (d),(e),(f) The same information for the S-edge. The PDoSs are calculated using a broadening parameter $\eta=0.1 \mathrm{eV}$, Eq. 5.1, and are plotted with a linear grayscale.

$\mathrm{eV}$ in total.

Figures 5.11(b) and (f) show the $k$-resolved and $k$-integrated densities of states of the Mo-edge. There are two prominent edge bands with energies in the $\mathrm{MoS}_{2}$ band gap. One edge band emerges from the bulk valence band at $k \approx 0.3$, and disperses upward with increasing $k$ to $1 \mathrm{eV}$. A second band disperses downward from the conduction band to $1.6 \mathrm{eV}$. The first edge band is also found in the threeband model, compare Figs. 5.6(b) and (f), albeit at a slightly higher energy, such that it is completely isolated from the bulk valence band. The second edge band is absent in the three-band model. We will discuss the character of these bands in more detail below. Like in the three-band model, there are also edge states at other energies, for instance just below the highest valence band at $\sim-1.1 \mathrm{eV}$, or in the hybridization gaps of the conduction bands.

The $k$-resolved and $k$-integrated densities of states of the S-edge are given in Figs. 5.11(c) and (g). At energies in the $\mathrm{MoS}_{2}$ band gap there is one prominent edge 
state, which starts at $k=0$ in the conduction band, and disperses downward with increasing $k$ to $0.7 \mathrm{eV}$. The three-band model shows the same edge state with more or less the same dispersion, compare to Figs. 5.6(c) and (g). In the eleven-band model this state is somewhat more prominently isolated from the conduction band. For the S-edge the eleven-band model does not give additional even edge states in the band gap as compared to the three-band model.

Finally, Figs. 5.11(d) and (h) show the $k$-resolved and $k$-integrated densities of states of the armchair edge. There are three edge bands with energies in the $\mathrm{MoS}_{2}$ band gap. One band is situated at $-0.1-0.5 \mathrm{eV}$ and a second edge band lies at 0.9-1.5 $\mathrm{eV}$. These two edge bands roughly correspond to the ones that are found in the threeband model of the armchair edge, see Figs. 5.6(d) and (h). In the eleven-band model these two edge bands are found at a slightly lower energy in the gap. In addition the present model finds a third edge band in the gap, just below the conduction band at 1.7-1.9 eV.
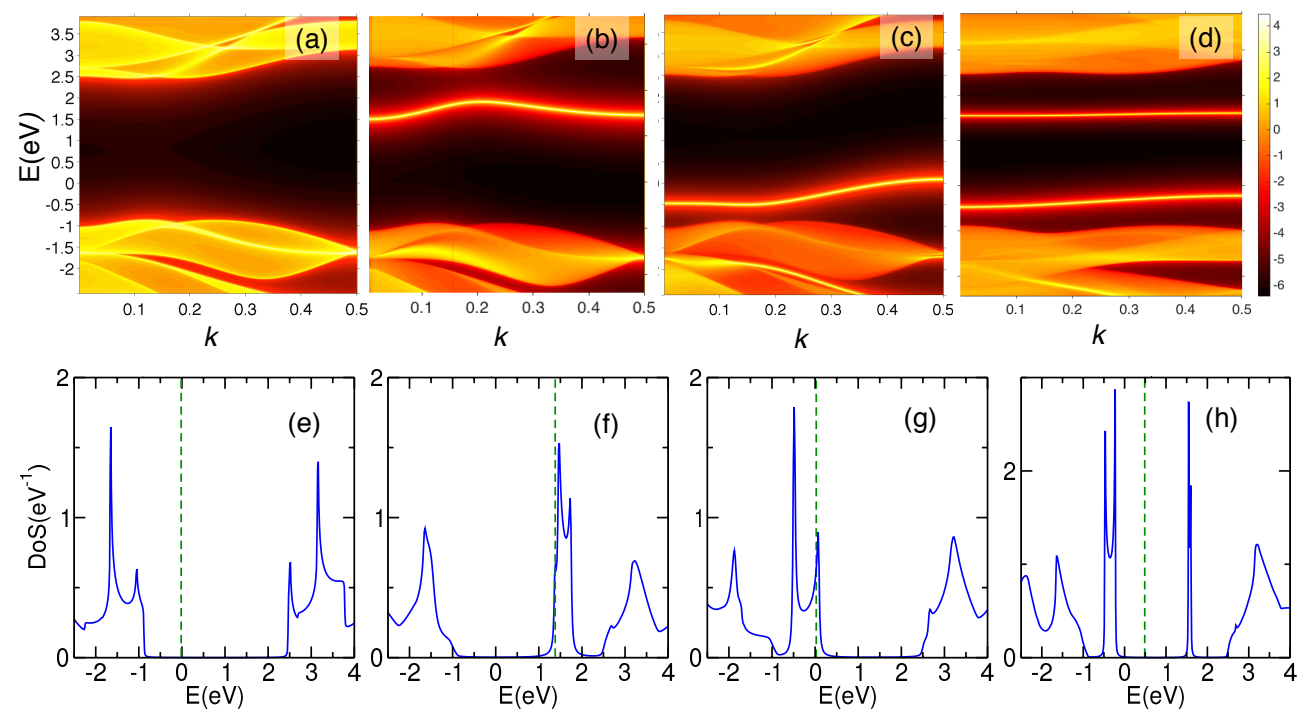

Figure 5.13: (Color online) As Fig. 5.11, but for the states with odd symmetry with respect to a mirror plane through the plane of the Mo atoms.

In conclusion, although there are quantitative differences between the electronic structures found with the eleven-band model and the three-band model, qualitatively they give similar results concerning the prominent edge states of even symmetry found in the $\mathrm{MoS}_{2}$ band gap. For the Mo-edge and the armchair edge, the eleven-band model gives an additional edge state, as compared to the three-band model, with energies just below the conduction band.

Figures 5.11(e)-(h) also show the CNLs. One needs of course all the bands to calculate the CNLs, including the odd bands to be discussed in the next section. A 
comparison to Fig. 5.6 reveals that the odd bands are in fact instrumental in fixing the CNLs. For instance, the CNLs of the Mo-edge and the S-edge differ by $1.4 \mathrm{eV}$, Figs. 5.11(f) and (g), whereas the corresponding CNLs in Figs. 5.6(f) and (g) differ by $0.3 \mathrm{eV}$ only. The eleven-band model gives one completely occupied edge band for the Mo-edge one one empty one, see Fig. 5.11(f), and for the S-edge it gives one completely empty edge band, see Fig. 5.11(g), which is unlike the partially filled edge bands found from the three-band model. As we will see in the next section, edge states of odd symmetry, which are absent in the three-band model, pin the CNLs of the Mo-edge and the S-edge, and make these edges metallic. The CNL at the armchair edge is in the gap between the two edge bands, see Fig. 5.11(h).

The orbital character of the edge states can be analyzed using the projected density of states, as calculated according to Eq. 5.5. The projections on the Mo $d_{3 z^{2}-r^{2}}$ orbital, the $d_{x y}$ and $d_{x^{2}-y^{2}}$ orbitals, and the $p$ orbitals of the $\mathrm{S}$ atoms are given in Figs. 5.12(a), (b), and (c), respectively, for the Mo-edge. The results indicate that both edge states in the $\mathrm{MoS}_{2}$ band gap have a mixed Mo and $\mathrm{S}$ character. The dominant Mo contributions clearly come from the $d_{x y}$ and $d_{x^{2}-y^{2}}$ orbitals. The upper edge state has $S p$ character mixed in, in particular for $k<0.4$, whereas the lower edge state has some $S p$ character mixed in for $k>0.4$. There is little $d_{3 z^{2}-r^{2}}$ character mixed in these edge bands, except at the band edges.

The projected densities of states of the S-edge, projected on the same orbitals, are given in Figs. 5.12(d), (e), and (f). Also here the edge state in the $\mathrm{MoS}_{2}$ band gap has a mixed Mo and $\mathrm{S}$ character. As for the Mo contribution, for $k=0$ the dominant character is Mo $d_{x y}$ and $d_{x^{2}-y^{2}}$. That changes somewhat for larger $k$; at $k=0.5$ the dominant character is Mo $d_{3 z^{2}-r^{2}}$. The contribution of the S-orbitals is fairly constant throughout the whole edge band.

\section{Odd bands}

The $k$-resolved and $k$-integrated bulk densities of states $n(k, E)$ corresponding to the odd states are given in Figs. 5.13(a) and (e). The band gap between the odd states is $3.3 \mathrm{eV}$, which is significantly larger than the gap between the even states. The top of the highest valence band of the odd states is approximately $0.8 \mathrm{eV}$ below the top of the highest valence band of the even states, whereas the bottom of the lowest conduction band of the odd states is approximately $0.6 \mathrm{eV}$ above that of the even states.

The $k$-resolved densities of state of the odd bands are shown in Figs. 5.13(b) and (c) for the Mo-edge and of the S-edge, respectively, and the corresponding $k$ integrated densities of states are shown in Figs. 5.13(f) and (g). Both edges have a prominent edge band with moderate dispersion inside the gap between the odd states. The edge band of the Mo-edge lies close to the conduction band between 1.3 and $1.8 \mathrm{eV}$, whereas the edge band of the S-edge is close to the top of the valence band between -0.5 and $0.2 \mathrm{eV}$.

If we also take the bulk band structure of the even bands into consideration, 

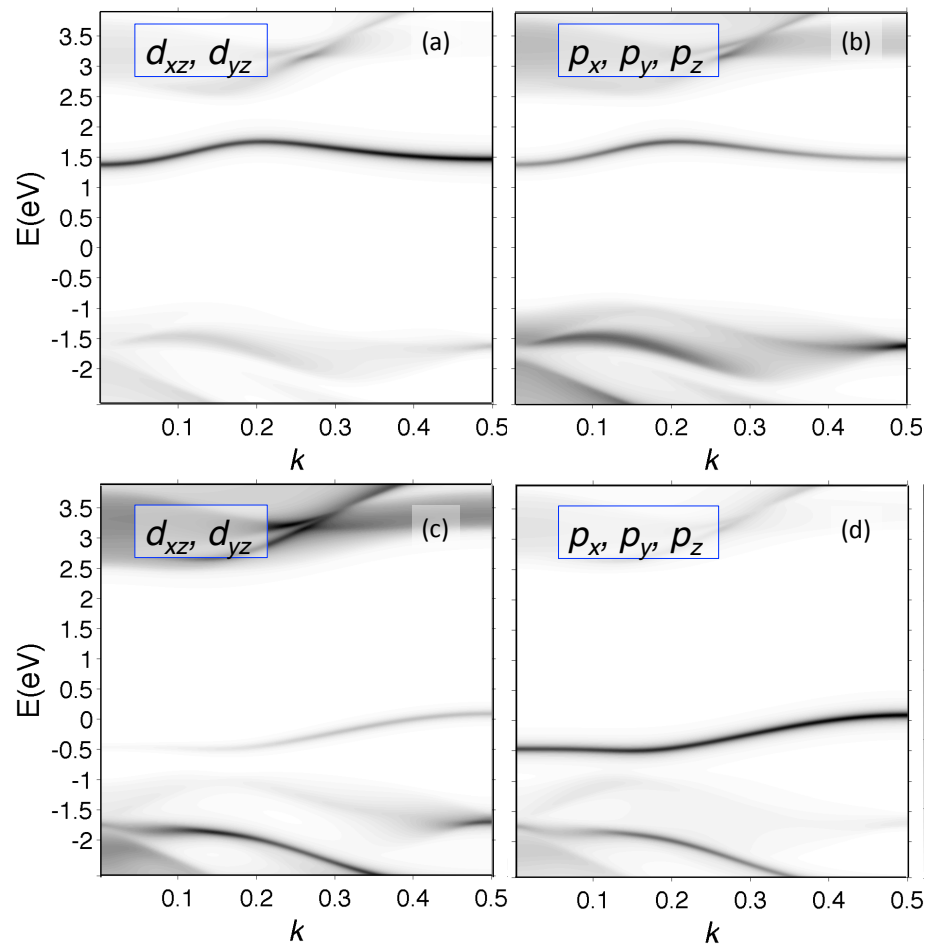

Figure 5.14: (Color online) As Fig. 5.12, but for the states with odd symmetry with respect to a mirror plane through the plane of the Mo atoms.

Figs. 5.11(a) and (d), then most of the edge band at the S-edge, Figs. 5.13(c) and (g) overlaps with the valence band. It is still a true edge state though, because the interaction between the odd edge state and the even bulk bands is symmetry forbidden. The edge band at the Mo-edge, Figs. 5.13(b) and (f) partially overlaps with the conduction band of the even states. Also this is a true edge state over the whole 1D Brillouin zone, as interaction with the bulk states is symmetry forbidden.

Figures 5.13(d) and (h) give the $k$-resolved and $k$-integrated densities of states corresponding to the armchair edge. These results show two edge bands in the gap region with a modest to small dispersion. The lowest of these edge bands has a dipersion of $0.4 \mathrm{eV}$, and taking also the even bulk bands into consideration, it lies in the $\mathrm{MoS}_{2}$ valence band. The upper edge band is near dispersionless; it is found in the gap at $1.6 \mathrm{eV}$.

The calculated CNLs are also given in Figs. 5.13(e)-(h). From these figures it becomes clear that the odd edge states are instrumental in controlling the CNLs of the edges. The CNL of the Mo-edge is found in the edge band that is close to the $\mathrm{MoS}_{2}$ conduction band, see Fig. 5.13(f), and the CNL of the S-edge lies in the edge band close to the $\mathrm{MoS}_{2}$ valence band, see Fig. 5.13(g). Within the eleven-band model both 
these edges are metallic in their charge neutral state. The CNL of the armchair edge lies between the two edge bands, Fig. 5.13(h), which means that the neutral armchair edge is semiconducting.

The orbital character of the odd symmetry edge states is identified using the projected densities of state. Figures 5.14(a) and (b) show the projections on the $d$ - and $p$-orbitals of the density of states of the Mo-edge, and Figs. 5.14(c) and (d) show the projections for the S-edge. The edge state at the Mo-edge has a mixed character, but it is still dominated by Mo $d$-orbitals. In contrast, the edge state at the S-edge has a clear $\mathrm{S} p$ character.

\subsection{Summary and Conclusions}

Growth of 2D semiconductors, such as the transition metal dichalcogenides $\mathrm{MX}_{2}$, commonly results in sheets that contain quasi-1D metallic structures, which are located at the edges of 2D crystallites, or at the boundaries between 2D crystal grains. Such edges and grain boundaries then form a practical realization of 1D metals. Metalic edges have also been identified as the active sites in $\mathrm{MX}_{2}$ catalysts. A standard technique for modeling edge or grain boundary states starts from nanoribbon structures. Nanoribbons involve the use of large supercells if one aims at obtaining converged results that enable the separation between edge and bulk properties. Moreover, as a nanoribbon has two edges, electron transfer between the edges can occur in order to equilibrate the system, which complicates identifying the properties of single edges.

In this paper we formulate a Green's function technique for calculating edge states of (semi-)infinite 2D systems with a single well-defined edge or grain boundary. We express bulk, edge and boundary Green's functions in terms of Bloch matrices. The latter are constructed from the solutions of a quadratic eigenvalue equation, which gives the traveling and evanescent Bloch states of the system. Electronic and structural reconstructions of edges or grain boundaries are easily incorporated in the technique. The formalism can be realized in any localized basis representation of the Hamiltonian.

Here we use it to calculate edge and grain boundary states of $\mathrm{MX}_{2}$ monolayers by means of tight-binding models. A simple three-band model is used to explore the electronic structures of the basic pristine zigzag and armchair edges in $\mathrm{MX}_{2}$. Within the three-band model the zigzag edges are metallic in their charge neutral state, whereas the armchair edge is semiconducting. We also analyze the electronic structures of $\mathrm{MoS}_{2}$ edges with a more general orientation, which are generally metallic, and the electronic structures of grain boundaries and of structurally modifed edges.

The three-band model captures part of the rich electronic structure of $\mathrm{MoS}_{2}$ edges, but more complete information is obtained from an eleven-band tight-binding model comprising the $d$ metal valence orbitals and the $p$ valence orbitals of the chalcogen atoms. Edge states in the band gap with even symmetry (with respect to a mirror 
through the plane of $\mathrm{M}$ atoms) are qualitatively similar to those found in the threeband model. The eleven-band model also gives edge states of odd symmetry in the band gap. The odd states are instrumental in fixing the charge neutrality level, and are most likely found at or near the Fermi level under experimental conditions. Charge neutral zigzag edges are metallic and the armchair edge is semiconducting. 


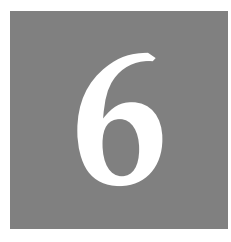

\section{One-dimensional edge states of 2D transition metal dichalcogenide nanoribbons}

We analyze the electronic structures of edges of transition metal dichalcogenide (TMD) nanoribbons using first-principles density functional theory calculations. We focus on edge states of $\mathrm{MoS}_{2}$ in particular. Pristine edges in zigzag orientation are metallic, whereas armchair edges are semiconducting. Saturating the Mo-edge with $S$ atoms annihilates danglingbond states, but preserves its metallic character. The same holds for reconstructions at the S-edge, which modify the number of edge states and their dispersions. Magnetic moments are created at the Mo atoms at the edges of some of the structures, either by exchange (Stoner) or by correlation (Hubbard) effects. The electronic structures of other TMD nanoribbons are similar to that of $\mathrm{MoS}_{2}$.

\subsection{Introduction}

As it becomes feasible to grow nanostructures and in-plane heterostructures of transition metal dichalcogenides $\left(\mathrm{MX}_{2} ; \mathrm{M}=\mathrm{Mo}, \mathrm{W}, \mathrm{X}=\mathrm{S}, \mathrm{Se}, \mathrm{Te}\right)$, the edges of these materials attract increasing attention [174, 170, 186, 218, 219]. The $\mathrm{MX}_{2}$ compounds are semiconducting, and their edges show a surprisingly rich structure of electronic states with energies in the $\mathrm{MX}_{2}$ band gap $[176,181]$. These states are localized on the first few atomic rows along the edges, and they have an one-dimensional character. Unlike the surface states of simple semiconductors, the edge states are often not associated simply with dangling bonds, but they reflect subtle changes in the electronic structure close to the edges $[177,187,220,221]$.

In many cases the edges are metallic, in particular for edge orientations as they 
are typically found in growth experiments [170,222]. The metallic edge states are not topologically protected, but they are not easily destroyed either. Even after saturating the edges with chalcogen atoms, and allowing for structural reconstructions, the edges generally remain metallic [223]. The states of pristine $\mathrm{MX}_{2}$ edges have been discussed in the previous chapter at the tight-binding level. Studying chemically or structurally modified edges is best done at the first-principles level, as structural optimization requires reliable calculations of total energies. At present we can do such calculations only on nanoribbons of a finite width, which has the drawback that the two edges of the nanoribbon can interact electronically.

The relatively low symmetry of the $\mathrm{MX}_{2}$ lattice $\left(D_{3 h}\right)$ implies that in many orientations nanoribbons have two chemically or structurally different edges with different electronic structures. Even if the ribbon is sufficiently wide, such that electronic states of the two edges do not overlap, one can still have a transfer of electrons between the edges to equilibrate the system. In addition, the $\mathrm{MX}_{2}$ lattice carries a polarization, which for terminations with polar edges creates an electric field across the ribbon that drives a transfer of electrons [41, 215, 224]. The inequivalence of the two edges complicates the interpretation of their electronic structure.

In the following we analyze the electronic structures of $\mathrm{MX}_{2}$ nanoribbon edges, obtained from first-principles density functional theory (DFT) calculations. We focus on $\mathrm{MoS}_{2}$ nanoribbons, and on the so-called zigzag edges in particular, which form the orientation that emerges most prominently in growth experiments. We consider pristine, i.e., bulk-terminated, edges and various chemical and structural modifications. Computational details and structures are discussed in Sec. 6.2; electronic structures are analyzed in Sec. 6.3. Some edge structures lead to possible magnetic effects, which is discussed toward the end of Sec. 6.3.

\subsection{Calculations}

We calculate electronic ground state energies within the framework of the projector augmented wave (PAW) method [45], as implemented in the Vienna Ab initio Simulation Package (VASP) [44, 46, 47], at the level of the DFT generalized gradient approximation (GGA), using the Perdew-Burke-Ernzerhof (PBE) functional [59]. The plane-wave kinetic-energy cutoff is set at $400 \mathrm{eV}$. The surface Brillouin zone is integrated using the Methfessel-Paxton technique [96] with a smearing parameter of 0.05 $\mathrm{eV}$, and a $k$-point sampling grid with a spacing of $0.01 \AA^{-1}$. Structures are optimized until the forces on the atoms are smaller than $0.01 \mathrm{eV} / \AA$. The energy convergence criterion is set at $10^{-5} \mathrm{eV}$.

$\mathrm{MoS}_{2}$ nanoribbons are modeled in a periodic supercell geometry. The supercell contains a ribbon of ten $\mathrm{MoS}_{2}$ units across (the $y$-direction in Fig.6.1), and one to six units along the edges (the $x$-direction) depending on the reconstruction. Periodic boundary conditions are used in all three directions. Ribbons in neighboring supercells are separated by $15 \AA(13 \AA)$ vacuum in the $z$ - (y)-direction. 
Side-view

S-edge

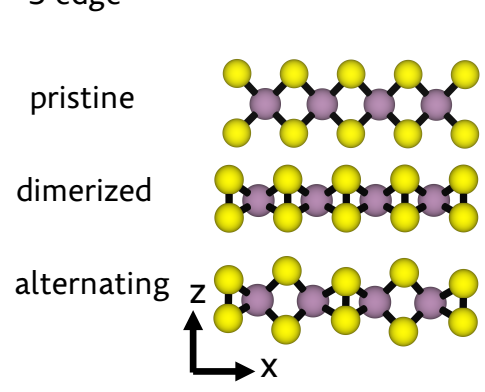

Top-view

Mo-edge

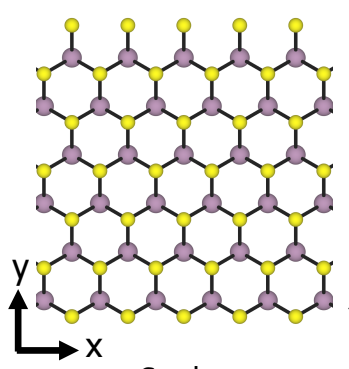

S-edge

\section{Side-view}

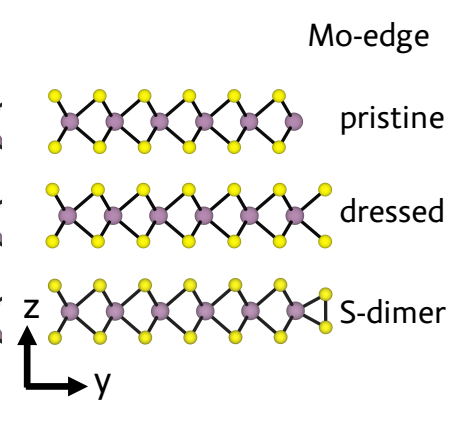

Figure 6.1: Middle: $\mathrm{MoS}_{2}$ nanoribbon with Mo and $\mathrm{S}$ zigzag edges along $x$; armchair edges are along $y$. Right, top: pristine Mo-edge, middle: dressed Mo-edge, bottom: dressed Mo-edge with dimerized S pairs. Left, top: pristine S-edge, middle: S-edge with dimerized S pairs, bottom: S-edge with alternating dimerized and undimerized S pairs.

\subsubsection{Edge structures}

Obviously the $D_{3 h}$ symmetry of a $\mathrm{MX}_{2}$ monolayer is broken in a ribbon geometry. All structures we are considering here however keep the mirror symmetry $\sigma_{h}$ with respect to a plane through the $\mathrm{M}$ atoms, and consequently all electronic states can be classified as even or odd with respect to $\sigma_{h}$. The edges of an hexagonal lattice with (10) and (12) orientation are called zigzag edges, respectively armchair edges, see Fig. 6.1. A nanoribbon with edges in armchair orientation has two identical edges. However, a nanoribbon with edges in zigzag orientation lacks an in-plane twofold symmetry, so the (10) and (10) edges are structurally different. The (10) edge is commonly called the M-edge, as in its pristine form it is terminated by metal atoms, see Fig. 6.1. Similarly the (10) edge is called the X-edge; it is terminated by chalcogen atoms.

The pristine M-edge exposes a row of metal atoms that have a coordination of four chalcogen atoms only. The metal edge atoms prefer to have sixfold coordination, allowing for the adsorption of two additional chalcogen atoms per metal edge atom, which is particularly favorable under chalcogen-rich growth conditions $[176,181]$. We called this the dressed M-edge. The incomplete coordination of the chalcogen atoms dressing the M-edge can be partially offset by dimerization of the chalcogen edge atoms, see Fig. 6.1. In fact, the undimerized form of the dressed M-edge is not stable, and dimerization occurs spontaneously during geometry optimization. Dimerization of the sulfur atoms at the Mo-edge of $\mathrm{MoS}_{2}$ decreases the 
total energy by $-0.43 \mathrm{eV} / \mathrm{S}$-dimer.

The chalcogen atoms at the X-edge can also dimerize. Here, however, it does not occur spontaneously, and the undimerized form is metastable. Nevertheless, the dimerized form of the S-edge of $\mathrm{MoS}_{2}$ is $-0.65 \mathrm{eV} /$ dimer lower in energy. Dimerization decreases the energy because new chemical bonds are formed, but it also introduces strain in the lattice at the X-edge. Edge structures that are only partially dimerized relieve this strain and can be lower in energy. A S-edge structure of $\mathrm{MoS}_{2}$ that consists of alternating dimerized and undimerized sulfur pairs, see Fig. 6.1, gives a structure that is $-0.95 \mathrm{eV} / \mathrm{S}$-dimer lower in energy than the fully dimerized form. In the following we discuss the electronic structures of pristine zigzag and armchair edges, dressed M-edges, and of pristine and reconstructed X-edges.

\subsection{Results}

\subsubsection{Pristine nanoribbons}

Figures 6.2(a) and (b) show the band structures of pristine $\mathrm{MoS}_{2}$ nanoribbons with edges in zigzag and in armchair orientations, respectively. The nanoribbon with edges in zigzag orientation contain a Mo-edge, as well as a S-edge. Consequently, the band structure contains the edge states associated with both these edges. In the following we use the colors red and blue for states that are localized at the Mo-edge, respectively the S-edge of the ribbon.

The zigzag nanoribbon shows four edge bands with energies inside the $\mathrm{MoS}_{2}$ gap. Two of these, numbered (1) and (2) in Fig. 6.2(a), belong to the Mo-edge. They are of even and odd symmetry, respectively. Projecting the corresponding wave functions on local atomic orbitals shows that these edge states have a large Mo $d$ contribution; $\left(d_{x y}, d_{x^{2}-y^{2}}, d_{3 z^{2}-r^{2}}\right)$ for the even states (1), and $\left(d_{x z}, d_{y z}\right)$ for the odd states (2). The latter has a dangling bond character, but the former is chemically more robust, as we will see in the next section. The two other bands, numbered (3) and (4) belong to the S-edge. Edge band (3) has even symmetry and it has a mixed S- $p$ and Mo- $d$ character, whereas edge band (4) has odd symmetry and has a dominant S- $p$ character. The latter is a dangling bond state with a significant $p_{z}$ contribution, hence its small dispersion.

These four edge bands are also found in the eleven-band tight-binding model used to describe edges of semi-infinite systems in the previous chapter. In some cases the tight-binding model gives these edge bands at slightly different energies. For most bands the differences with the DFT calculations are smaller than $0.5 \mathrm{eV}$, with the exception of the odd band (2) at the Mo-edge, where the DFT result for the nanoribbon is $\sim 1 \mathrm{eV}$ lower in energy, as compared to the tight-binding result. Shifts such as these may come from the difference in local potential experienced by edge and bulk atoms, which is an effect captured in the DFT, but not in the tight-binding, calculations.

The band dispersions obtained by DFT and tight-binding calculations are also 

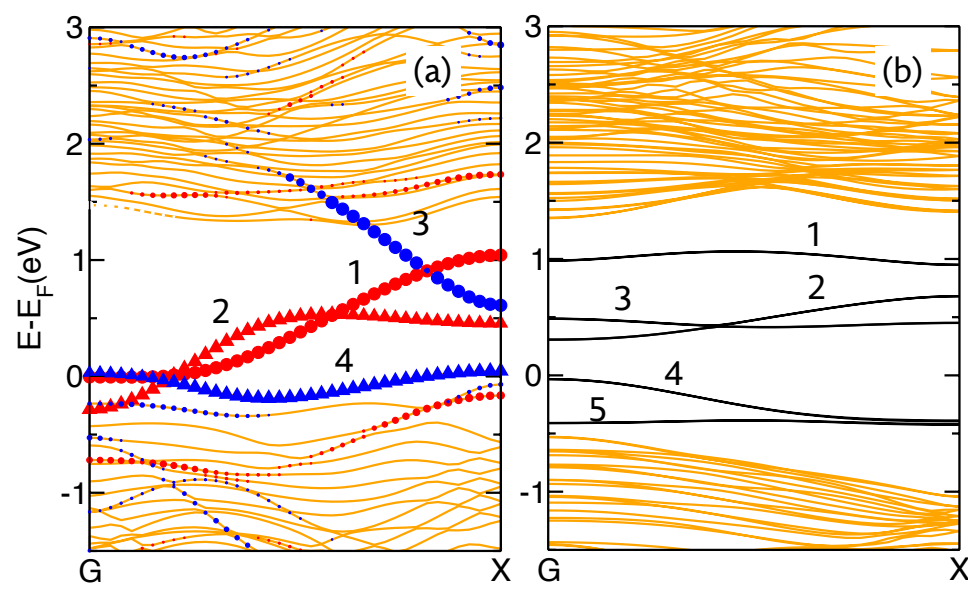

Figure 6.2: (a) The energy bands of a pristine $\mathrm{MoS}_{2}$ nanoribbon in zigzag orientation. Red and blue colors indicate edge bands resulting from the Mo-edge and the S-edge, respectively. The size of the symbols reflects projections of the wave functions on the $\mathrm{MoS}_{2}$ unit at the edges. Circles indicate even states, and triangles indicate odd states with respect to $\sigma_{h}$ symmetry. The zero of energy is set at the Fermi level. (b) The energy bands of a pristine $\mathrm{MoS}_{2}$ nanoribbon in armchair orientation with the edge bands in the gap indicated in black.

slightly different, yet the agreement between the two is sufficiently strong to use the tight-binding results of the previous chapter for identifying the states of the nanoribbon. The tight-binding model shows additional edge bands with energies close to the $\mathrm{MoS}_{2}$ band edges, for instance, two states of even symmetry at the Mo-edge, one close to the bottom of the conduction band, and one not far from the top of the valence band. With some effort these states can also be identified in the electronic structure of the nanoribbon, Fig. 6.2(a).

The band structure of a nanoribbon terminated by armchair edges, is shown in Fig. 6.2(b). For a nanoribbon in armchair orientation the two edges are equivalent, and they give identical edge states (provided the ribbon is sufficiently wide). The electronic structure reveals five bands per edge in the gap region, which agrees well with the tight-binding results for the armchair edge, discussed in the previous chapter. Bands (1), (2) and (4) have even symmetry, and bands (3) and (5) have odd symmetry. Bands (2) and (4) have a dispersion of $\sim 0.4 \mathrm{eV}$, and have a mixed Mo$d$ and S- $p$ character. The remaining three bands have a very small dispersion and their states are localized on the edge atoms. Bands (1) and (3) have a dominant Mo- $d$ character, whereas band (5) has a dominant S- $p$ character.

According the the DFT calculations, the pristine nanoribbon in zigzag orientation is metallic, whereas that in armchair orientation is semiconducting. The position of the Fermi level in the armchair nanoribbon corresponds to the charge neutrality level of the armchair edge found in the tight-binding calculation discussed in the previous 

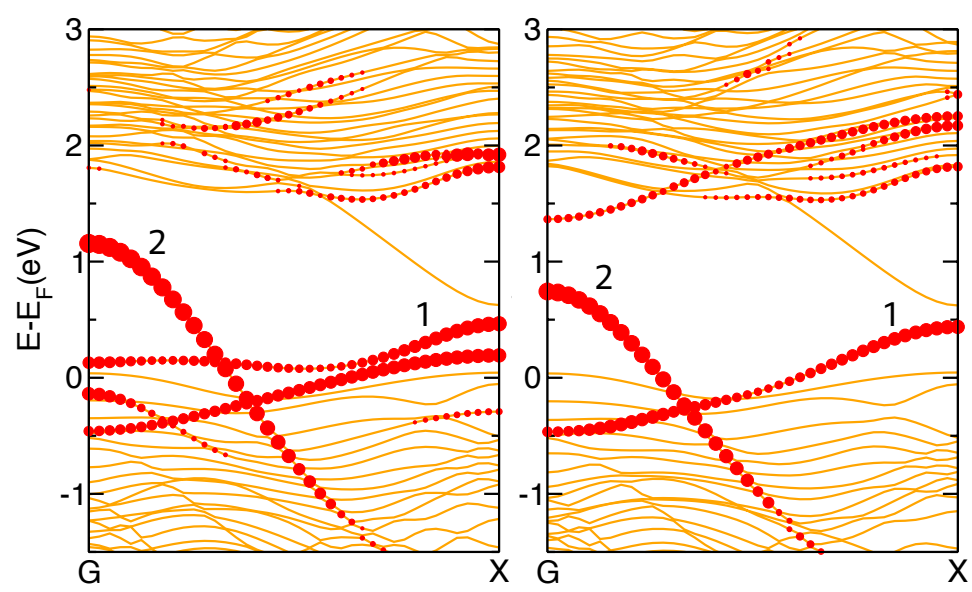

Figure 6.3: (a) The energy bands of a $\mathrm{MoS}_{2}$ nanoribbon with a dressed Mo-edge. The size of the red circles reflects projections of the wave functions (even or odd) on the $\mathrm{MoS}_{2}$ unit at the Mo-edge. The zero of energy is set at the Fermi level. (b) The same for a ribbon with dimerized $S$ pairs at the dressed Mo-edge.

chapter. For the zigzag nanoribbon the Fermi level is close to valence band. If one starts from the positions of the charge neutrality levels of the Mo- and the S-edge calculated with tight-binding, this suggests that equilibration between the edges of the nanoribbon should give a transfer of electrons from the Mo- to the S-edge. The position of the Fermi level in Fig. 6.2(a) is consistent with that.

\subsubsection{Dressed Mo-edges}

Dressing the Mo-edge of a nanoribbon with $\mathrm{S}$ atoms in bulk-like positions gives the band structure shown in Fig. 6.3(a). Not surprisingly, the band structure of the Moedge is strongly modified. Most prominent is an edge band (2) that starts above midgap at $k=0$ and disperses downward with increasing $k$ into the valence band. The corresponding states are almost completely localized on the sulfur atoms dressing the Mo-edge. The states have odd symmetry and are composed mainly of S- $p_{x}$ orbitals (with $x$ the direction parallel to the edge, Fig. 6.1), see Fig. 6.4. The edge band show a dispersion that is typical of a $p \sigma$-interaction, with the high energy antibonding state at $k=0$, and increasing bonding character (decreasing energy) with increasing $k$.

Below the Fermi level the edge band extends into the $\mathrm{MoS}_{2}$ valence band, where due to its interaction with states belonging to the interior of the nanoribbon, it becomes a resonance. From the part of the edge band that can be traced one estimates its total width as $\sim 3 \mathrm{eV}$. In addition we find in Fig. 6.3(a) a pair of edge bands (1) close to the top of the valence band. The pair can be interpreted as one band dispersing from -0.5 to $0.5 \mathrm{eV}$, crossing an almost dispersionless band. The latter is likely a 


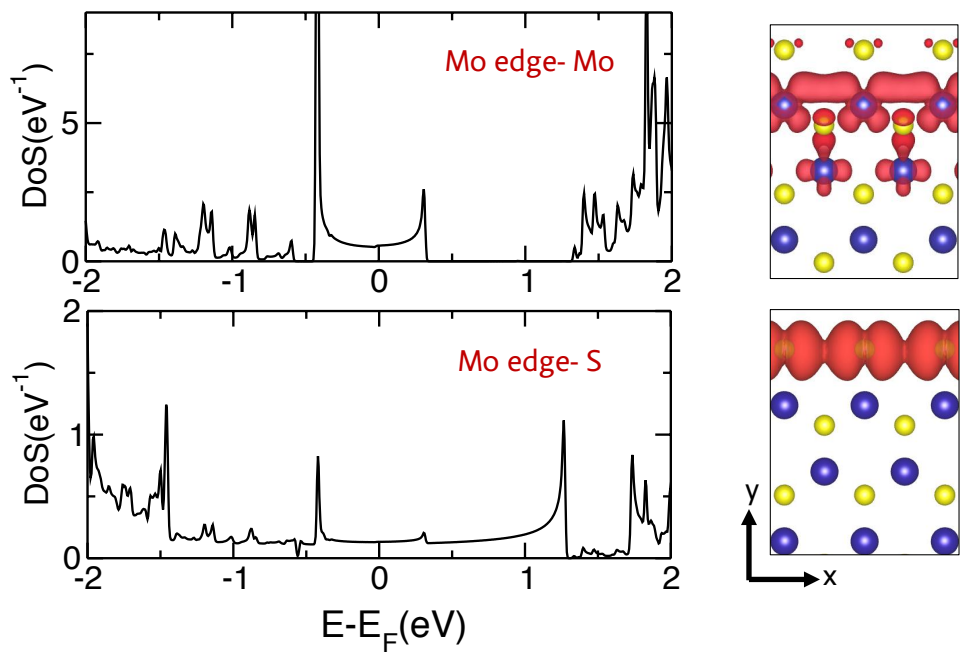

Figure 6.4: (a) Density of states (DoS) of a $\mathrm{MoS}_{2}$ nanoribbon with a dressed Mo-edge, projected on the row of Mo atoms at the edge. (b) DoS projected on the dressing $S$ atoms at the Mo-edge. (c) Local DoS at $E=0$. (d) Local DoS at $E=1 \mathrm{eV}$.

S dangling bond state with a significant $p_{z}$ contribution, similar to band (4) in Fig. 6.2 (a). The character of band (1) is very similar to edge band (1) found for the pristine Mo-edge, Fig. 6.2(a).

Optimizing the structure of the dressed Mo-edge results in a dimerization of the dressing $S$ atoms, see Fig. 6.1. The resulting band structure is shown in Fig. 6.3(b). The prominent edge band (2) remains, but it is shifted downward in energy by $0.5 \mathrm{eV}$, as compared to the edge with undimerized $\mathrm{S}$ atoms shown in Fig. 6.3(a). The character of the band remains unchanged upon dimerization, i.e., its has odd symmetry and is formed by a $\sigma$-interaction of S- $p_{x}$ orbitals.

Of the pair of bands (1) of the undimerized dressing, only one edge state remains in the dimerized case, which is the band with a dispersion $\sim 1 \mathrm{eV}$ that is similar to edge band (1) of the pristine Mo-edge. It should be emphasized that the edge states at the dressed Mo-edge with dimerized S pair, are not a consequence of dangling bonds, since the edge atoms are now fully coordinated.

To further characterize the edge bands (1) and (2), Figs. 6.4(a) and (b) show the density of states projected on the Mo and S atoms at the Mo-edge. Edge state (1) has a large contribution from the Mo atom and no contribution from the $S$ atoms at the edge, see Fig. 6.4(a). The Van Hove singularities at the band edges $( \pm 0.4 \mathrm{eV})$ vividly demonstrate the one-dimensional character of the edge states. In contrast, edge state (2) has a large contribution from the edge $S$ atoms, Fig. 6.4(b).

*The latter also seems to have some contribution from the Mo atoms, but this is an artefact coming from the projection method. Projections in VASP proceed by decomposing each plane wave in the basis set into spherical partial waves in a sphere around an atom. The radius of such a sphere is of course not 
Plotting the wave function densities of these two edge states confirms their character. An example of a wave function density belonging to a state at $E=0 \mathrm{eV}$ in edge band (1) is given in Fig. 6.4(c). It contains a bonding interaction between $d_{x y}$ orbitals of neighboring Mo atoms, and an anti-bonding interaction with $p_{y}$ orbitals on the second row of $\mathrm{S}$ atoms and the $d_{x^{2}-y^{2}}$ orbitals of the second row of Mo atoms. Remarkably the density has virtually no contribution from the $S$ atoms right at the edge.

As mentioned before, the character of edge state (1) of the dressed Mo-edge is very similar to that of edge state (1) of the pristine Mo-edge. The main difference is that edge state (1) in the dressed Mo-edge is $0.5 \mathrm{eV}$ lower in energy than in the pristine Mo-edge. It suggests that the presence in the dressed Mo-edge of an outer row of $S$ atoms results in a more attractive potential at the Mo atomic sites, which decreases the energy of edge state (1).

An example of a wave function density belonging to a state at $E=1 \mathrm{eV}$ in edge band (2) is given in Fig. 6.4(d). It is consistent with an anti-bonding $\sigma$-interaction between $p_{x}$ orbitals on neighboring $\mathrm{S}$ atoms, as expected.

\subsubsection{Reconstructed S-edges}

Pristine S-edges are not dressed under clean growth conditions, but they are subjected to reconstructions. A reconstruction consisting of alternating dimerized and undimerized S pairs at the edge, Fig. 6.1, yields the band structure shown in Fig. 6.5(a). Dimerizing all the $S$ atoms at the edge gives the band structure shown in Fig. 6.5(b). In both these cases the band structures have changed considerably, as compared to the band structure of the undimerized S-edge, Fig. 6.2(a), apart from the trivial folding of the bands resulting from doubling the period by the reconstruction.

For the fully dimerized case there is only one folded edge band (3) with energies in the $\mathrm{MoS}_{2}$ gap, as compared to the two bands (3) and (4) at the S-edge for the undimerized case. Dimerization has removed edge band (4) (S- $p$ character, odd symmetry) from the gap region, which in the undimerized case we have identified as corresponding to dangling bonds. In addition, dimerization has lowered band (3) (mixed Mo- $d$, S- $p$ character, even symmetry) in energy. The Fermi level now cuts through edge band (3), and it is the band that determines the metallicity of the Sedge.

As discussed in Sec 6.2.1, a reconstruction consisting of alternating dimerized and undimerized S pairs, has a lower energy than the fully dimerized or the fully undimerized forms. The band structure corresponding to this alternating structure, Fig. 6.5(a), shows three edge bands with little dispersion in the band gap. Edge band (4), which stems from the undimerized S pairs, reappears, and edge band (3) splits up into two bands, $(3)$ and $\left(3^{\prime}\right)$. The band (3) at the Fermi level corresponds to states that are localized on the dimerized $S$ pairs (and the Mo atoms at the edge, see below),

uniquely defined. If choosing it small, one throws away most of the information. Choosing it large results in picking up information belonging to neighboring atoms. 

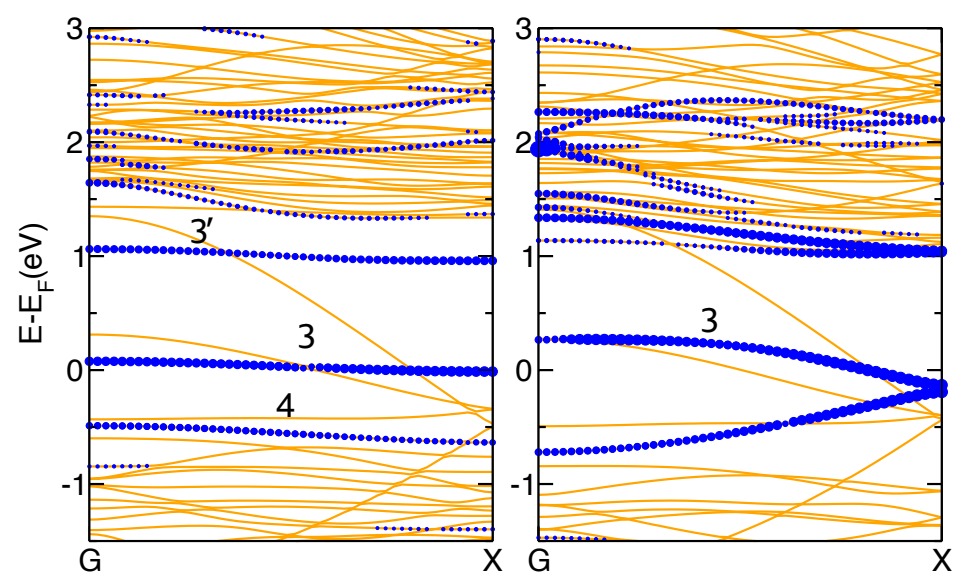

Figure 6.5: (a) The energy bands of a $\mathrm{MoS}_{2}$ nanoribbon with a reconstructed S-edge, consisting af alternating dimerized and undimerised $S$ pairs. The size of the blue circles reflects projections of the wave functions (even or odd) on the $\mathrm{MoS}_{2}$ unit at the S-edge. The zero of energy is set at the Fermi level. (b) The same for a ribbon with all dimerized $S$ pairs at the S-edge.

whereas the edge band $\left(3^{\prime}\right)$ at $\sim 1 \mathrm{eV}$ corresponds to states that are localized on the undimerized dimerized $S$ pairs (and the Mo atoms at the edge). All of these edge bands have a small dispersion, which means we are dealing with localized states.

The density of states, projected on the Mo and S atoms at the S-edge with this reconstruction, is given in Figs. 6.6(a) and (b). It clearly shows that edge band (4) has $S$ character, whereas band (3) and ( $\left.3^{\prime}\right)$ have a mixed Mo and $S$ character. Of the latter two, band (3) has a dominant Mo character, whereas for band ( $\left.3^{\prime}\right)$ the Mo and $S$ contributions are more evenly matched. The wave function density belonging to a state in band (3) is given in Fig 6.6(b). It clearly shows a state that is localized on the dimerized $S$ pair, and the pair of Mo atoms bonded to it. The state has even symmetry and has dominant contributions from Mo $d_{3 z^{2}-r^{2}}$ and $d_{x^{2}-y^{2}}$, and $\mathrm{S} p_{x}$.

\subsubsection{Magnetic reconstructions}

Bulk $\mathrm{MoS}_{2}$ is nonmagnetic, but the edge states shown in Fig. 6.6 have a very small band width, which makes it worth while to investigate whether this S-edge is subjected to magnetic instabilities resulting from exchange or on-site Coulomb interactions. A spin-polarized calculation gives no exchange splitting for the edge states at the dressed Mo-edge, Fig. 6.4, but it gives a sizable splitting for the states at the reconstructed S-edge. The result is shown in Fig. 6.7. The flat band at the Fermi level splits up into spin-up and spin-down bands with an exchange splitting of $\sim 0.4 \mathrm{eV}$, and the band at $\sim 1 \mathrm{eV}$ acquires an exchange splitting roughly half that size.

The partially filled edge state at the Fermi level results in local magnetic mo- 


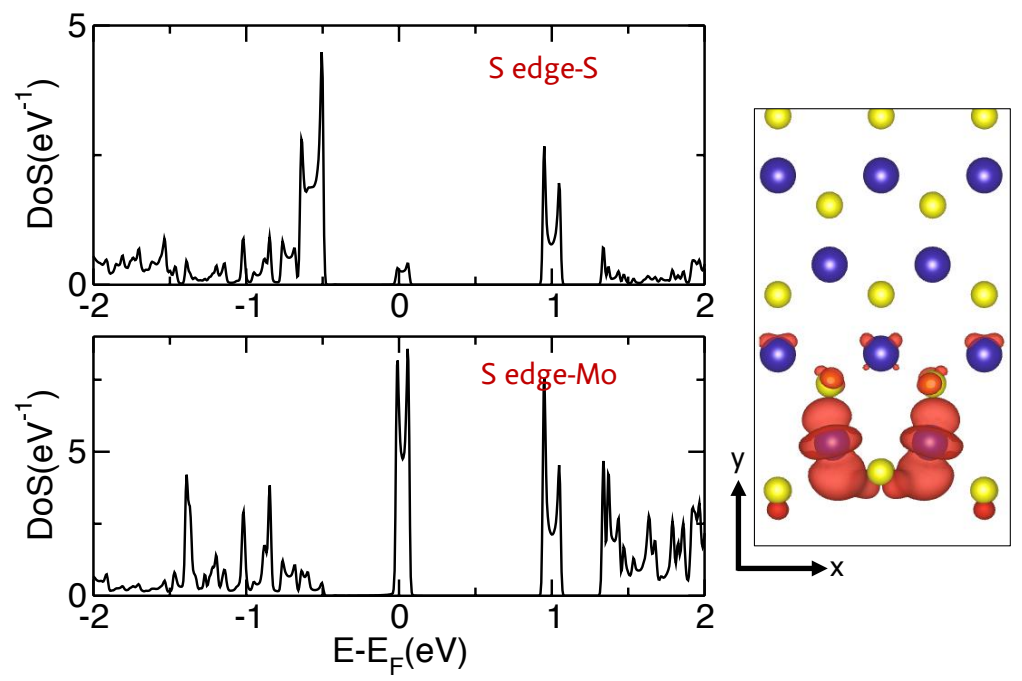

Figure 6.6: (a) Density of states (DoS) of a $\mathrm{MoS}_{2}$ nanoribbon with a reconstructed S-edge of alternating dimerized and undimerized $S$ pairs, projected on the row of $S$ atoms at the edge. (b) DoS projected on the Mo atoms at the S-edge. (c) Local DoS at $E=0$.

ments, which are almost exclusively carried by the Mo atoms at the reconstructed edge, see Fig. 6.6(b) The calculated magnetic moments on these Mo atoms are 0.31 $\mu_{B}$. The $\mathrm{S}$ atoms of the dimerized pairs at the edge carry a very small magnetic moment of $-0.03 \mu_{B}$, whereas the undimerized $S$ atoms have a zero magnetic moment. The lack of dispersion of the polarized edge state at the Fermi level clearly indicates that the magnetic moments are localized. It suggests that the coupling between those moments is small, so that the paramagnetic state is much more likely to form than any long-range magnetic ordering. A purely one-dimensional system would not have a long-range ordering anyway, of course (according to the Mermin-Wagner theorem).

For flat bands such as they are found at the reconstructed S-edge one may expect that electron correlations due to on-site Coulomb interactions are relatively important. In particular, the edge state at the Fermi level with a close to $1 / 3$ electron per Mo atom suggests the possibility of a Mott-Hubbard transition and a mixed-valence compound, if one triples the unit cell along the edge. We study this possibility by GGA $+U$ calculations on a nanoribbon with $3 \times$ periodicity along the edge, with a Hubbard $U=1$ to $4 \mathrm{eV}$ on the Mo atoms. No qualitative changes from the homogeneous state described in the previous paragraph are found, i.e., no tendency to form Mo atoms with different valences. For a sufficiently large $U$ the Mo-edge also becomes spin-polarized, as edge band (1) splits up into a lower and an upper Hubbard band, Fig. 6.5(b). A more definite conclusion requires an explicit calculation of $U$ in this structure, which is outside the scope of the present study. 

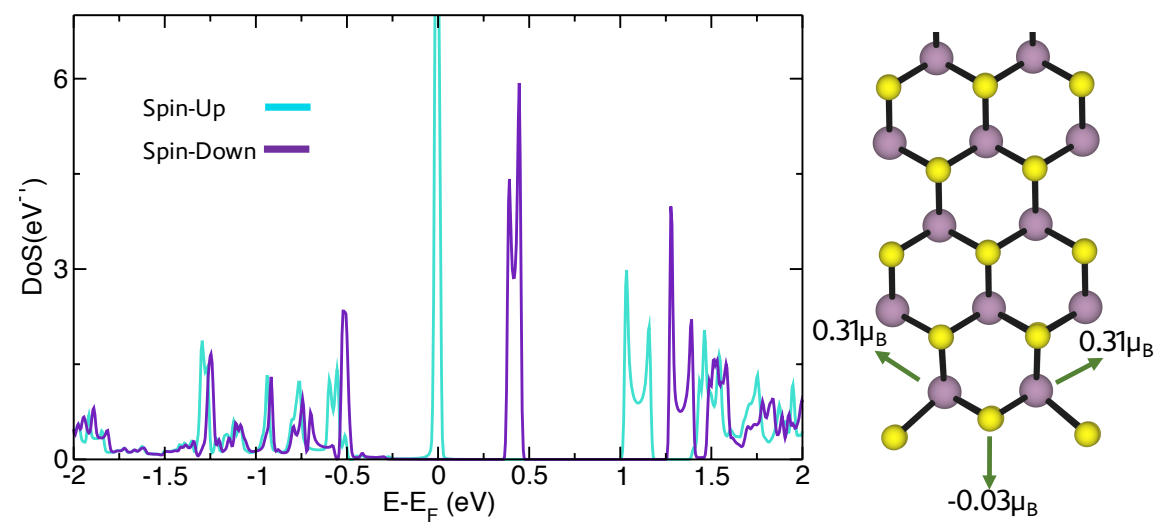

Figure 6.7: (a) Spin-polarized DoS of a $\mathrm{MoS}_{2}$ nanoribbon with a reconstructed S-edge of alternating dimerized and undimerized $S$ pairs, projected on the row of $S$ atoms at the edge. (b) Magnetic moments on the edge atoms.

We also studied the influence of spin-orbit coupling on the edge states in $\mathrm{MoS}_{2}$. The corresponding band structure of a nanoribbon with a dressed Mo-edge, and a reconstructed S-edge, is shown in Fig. 6.8 (here $U$ is set to zero again). The Mo-edge shows the edge bands (1) and (2) we have identified Fig. 6.3(b). The bands are folded in Fig. 6.8, because we have doubled the unit cell along the edge. Spin-orbit coupling splits band (1) by 50-60 meV, but it hardly splits band (2) at all. This is consistent with the character of these bands. The states of band (1) have a sizable Mo- $d$ character, whereas those in band (2) have a pure S- $p$ character, see Fig. 6.4. Obviously the spinorbit coupling on the Mo atom is much larger than that on the $\mathrm{S}$ atom.

The bands at the S-edge are already spin-split before we apply spin-orbit coupling, see Fig. 6.7. Bands (3) and (3'), see Fig. 6.5(a), remain spin-split, and are only slightly shifted by spin-orbit coupling. These bands have important Mo- $d$ contributions. Band (4) is hardly affected at all, as it is mainly composed of S- $p$ states.

\subsubsection{Other $\mathrm{MX}_{2}$ compounds}

$\mathrm{MX}_{2}(\mathrm{M}=\mathrm{Mo}, \mathrm{W}, \mathrm{X}=\mathrm{S}, \mathrm{Se}, \mathrm{Te})$ monolayers have the same basic structure as $\mathrm{MoS}_{2}$ and similar chemical properties. Therefore, one may expect the electronic structure of their nanoribbon edges to be similar to that of $\mathrm{MoS}_{2}$. This is indeed the case, as we see in Fig. 6.9. The dressed M-edges of $\mathrm{MoSe}_{2}$ and $\mathrm{WS}_{2}$ show the edge bands (1) and (2) identified for $\mathrm{MoS}_{2}$ in Fig. 6.3(b). In Fig. 6.9 these bands are folded, as we have used a unit cell along the edge of double size here. The states in bands (1) and (2) have the same character as the $\mathrm{MoS}_{2}$ bands shown in Fig. 6.4. States in band (1) have even symmetry, and have a considerable M- $d$ character. The corresponding band has a moderate dispersion of $0.7-1.0 \mathrm{eV}$. States in band (2) have odd symmetry, and foremost consist of the $p_{x}$ orbitals of the $\mathrm{X}$ atoms dressing the edge. Band (2) has 


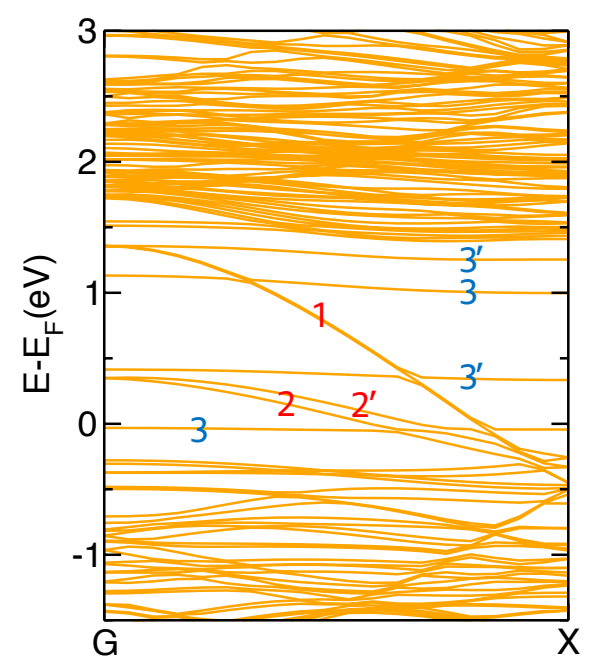

Figure 6.8: The energy bands of a $\mathrm{MoS}_{2}$ nanoribbon with a dresssed Mo-edge and a reconstructed S-edge, including spin-orbit coupling.

a dispersion $\sim 3 \mathrm{eV}$; only the top half of the band lies within the band gap.

The reconstructed X-edges display the edge bands (3) and (4) we found for $\mathrm{MoS}_{2}$ in Fig. 6.5(a). The states in band (3) have the character shown in Fig 6.6, and have strong M- $d$ contributions. They are susceptible to possible magnetic instabilities, as
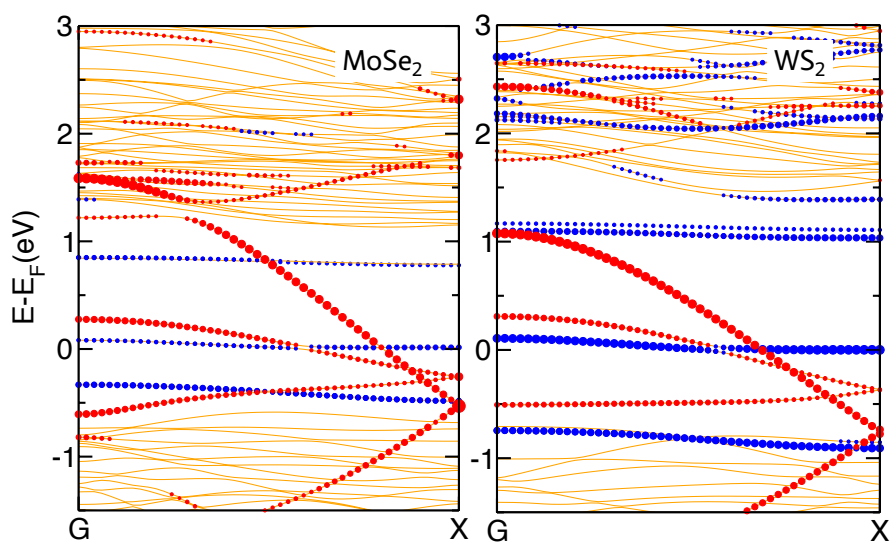

Figure 6.9: (a) The energy bands of a $\mathrm{MoSe}_{2}$ nanoribbon with a dressed Mo-edge and a reconstructed Se-edge. The size of the blue and red circles reflect projections of the wave functions on the $\mathrm{MoSe}_{2}$ unit at the Se-edge and Mo-edge respectively. The zero of energy is set at the Fermi level. (b) The same for a $\mathrm{WS}_{2}$ nanoribbon. 
discussed in the previous section. Band (4) has a X-atom dangling-bond character. 


\section{Tight-binding model}

\section{A.1 Three-band tight-binding model}

Following Mattheis, the three-band tight binding model discussed in Sec 5.2.3 only explicitly uses the sublattice of $\mathrm{M}$ atoms and the $d$-orbitals of $A_{1}^{\prime}$ and $E^{\prime}$ symmetry, i.e., the orbitals that are symmetric with respect to $\sigma_{h}$, mirror symmetry in the $\mathrm{MoS}_{2}$ plane [151]. We number these orbitals as

$$
d_{3 z^{2}-r^{2}}=|0\rangle, d_{x y}=|1\rangle, d_{x^{2}-y^{2}}=|2\rangle \text {. }
$$

The matrix blocks $\mathbf{H}_{p, q}$ discussed in Sec. 5.2.2 are then $3 \times 3$ matrices, where $\mathbf{H}_{p, q}$ denotes the real space Hamiltonian matrix block pertaining to the interaction between atoms in the unit cell at the origin and atoms in the unit cell at $p \mathbf{a}_{1}+q \mathbf{a}_{2}$. Using only nearest neighbor interactions, one has $|p|,|q| \leq 1$. Defining the tight-binding parameters $\epsilon_{i}=\langle i|h| i\rangle ; i=0,1,2$ and $t_{i j}=\langle i|h| j\rangle ; i, j=0,1,2$, and making use of the rotation properties of the $d$-orbitals, one derives the matrix blocks

$$
\begin{aligned}
\mathbf{H}_{0,0}= & {\left[\begin{array}{ccc}
\epsilon_{0} & 0 & 0 \\
0 & \epsilon_{1} & 0 \\
0 & 0 & \epsilon_{2}
\end{array}\right] ; } \\
\mathbf{H}_{1,0}= & {\left[\begin{array}{ccc}
t_{00} & \frac{\sqrt{3}}{2} t_{02}+\frac{1}{2} t_{01} & -\frac{1}{2} t_{02}+\frac{\sqrt{3}}{2} t_{01} \\
\frac{\sqrt{3}}{2} t_{02}-\frac{1}{2} t_{01} & \frac{1}{4} t_{11}+\frac{3}{4} t_{22} & \frac{\sqrt{3}}{4}\left(t_{11}-t_{22}\right)-t_{12} \\
-\frac{1}{2} t_{02}-\frac{\sqrt{3}}{2} t_{01} & \frac{\sqrt{3}}{4}\left(t_{11}-t_{22}\right)+t_{12} & \frac{1}{4} t_{22}+\frac{3}{4} t_{11}
\end{array}\right] ; }
\end{aligned}
$$




$$
\begin{aligned}
\mathbf{H}_{0,1}= & {\left[\begin{array}{ccc}
t_{00} & -t_{01} & t_{02} \\
t_{01} & t_{11} & -t_{12} \\
t_{02} & t_{12} & t_{22}
\end{array}\right] ; } \\
\mathbf{H}_{1,1}= & {\left[\begin{array}{ccc}
t_{00} & -\frac{\sqrt{3}}{2} t_{02}-\frac{1}{2} t_{01} & -\frac{1}{2} t_{02}+\frac{\sqrt{3}}{2} t_{01} \\
-\frac{\sqrt{3}}{2} t_{02}+\frac{1}{2} t_{01} & \frac{1}{4} t_{11}+\frac{3}{4} t_{22} & -\frac{\sqrt{3}}{4}\left(t_{11}-t_{22}\right)+t_{12} \\
-\frac{1}{2} t_{02}-\frac{\sqrt{3}}{2} t_{01} & -\frac{\sqrt{3}}{4}\left(t_{11}-t_{22}\right)-t_{12} & \frac{1}{4} t_{22}+\frac{3}{4} t_{11}
\end{array}\right] . }
\end{aligned}
$$

The coupling associated with matrix elements $t_{00}, t_{02}, t_{22}, t_{11}$ is even with respect to inversion and the one associated with $t_{01}, t_{12}$ is odd. It is easy to see that

$$
\mathbf{H}_{-p,-q}=\left(\mathbf{H}_{p, q}\right)^{T}
$$

The values of the tight-binding parameters are taken from Liu et al.,[204] which have been obtained by fitting the tight-binding band structure of a $\mathrm{MX}_{2}$ sheet to a DFT GGA/PBE band structure [59], using the highest valence band and the two lowest conduction bands in the fit. GGA/PBE gives an optimized lattice parameter of $3.19 \AA$ for $\mathrm{MoS}_{2}$, which is 1-2 \% larger than the reported experimental values [109, 110, 111]. With this lattice parameter the calculated band gap is $1.63 \mathrm{eV}$, which is smaller than the experimental optical band gap of $1.85 \mathrm{eV}$ [25].

\section{A.2 Eleven-band tight-binding model}

The eleven-band tight-binding model of $\mathrm{MX}_{2}$ uses a basis set composed of all five $d$-orbitals of the $\mathrm{M}$ atom, and the six $p$ orbitals of the two $\mathrm{X}$ atoms. Following the approach of Cappelluti et al.,[104, 225] we include next nearest neighbor interactions, and use the Slater-Koster two-center approximation for the hopping matrix elements [226]. The real space matrix blocks $\mathbf{H}_{p, q}$ discussed in Sec. 5.2.2 then have the form

$$
\begin{aligned}
\mathbf{H}_{0,0} & =\left[\begin{array}{cc}
\boldsymbol{\epsilon}_{d} & \mathbf{t}_{d p}^{0,0} \\
\left(\mathbf{t}_{d p}^{0,0}\right)^{T} & \boldsymbol{\epsilon}_{p}
\end{array}\right], \mathbf{H}_{1,0}=\left[\begin{array}{cc}
\mathbf{t}_{d d}^{1,0} & \mathbf{0} \\
\mathbf{0} & \mathbf{t}_{p p}^{1,0}
\end{array}\right], \mathbf{H}_{0,1}=\left[\begin{array}{cc}
\mathbf{t}_{d d}^{0,1} & \mathbf{0} \\
\left(\mathbf{t}_{d p}^{0,1}\right)^{T} & \mathbf{t}_{p p}^{0,1}
\end{array}\right], \\
\mathbf{H}_{1,1} & =\left[\begin{array}{cc}
\mathbf{t}_{d d}^{1,1} & \mathbf{0} \\
\left(\mathbf{t}_{d p}^{1,1}\right)^{T} & \mathbf{t}_{p p}^{1,1}
\end{array}\right],
\end{aligned}
$$

with the remaining blocks constructed according to Eq. A.4. The bulk Hamiltonian for an infinite layer with two-dimensional translation symmetry is written in this notation as

$$
\mathbf{H}\left(k_{1}, k_{2}\right)=\mathbf{H}_{0,0}+\mathbf{A}+\mathbf{A}^{\dagger} ; \quad \mathbf{A}=\mathbf{H}_{1,0} e^{i 2 \pi k_{1}}++\mathbf{H}_{0,1} e^{i 2 \pi k_{2}}++\mathbf{H}_{1,1} e^{i 2 \pi\left(k_{1}+k_{2}\right)} .
$$


The parameters in the model can be found by fitting the tight-binding band structure obtained with this bulk Hamiltonian to a band structure obtained from a DFT calculation. The model turns out to be too restrictive to obtain a satisfactory fit for all eleven bands with one parameter set. A good fit can however be obtained if we divide the bands into a set of even symmetry, and a set of odd symmetry, and use different parameters for the two sets. Mirror symmetry $\sigma_{h}$ in the $\mathrm{MX}_{2}$ plane holds for monolayers, as well as for edges, so states that are even or odd with respect to $\sigma_{h}$, can be treated separately. In Sec. A.2.1 we give the expressions for the matrix blocks $\boldsymbol{\epsilon}_{a}$ and $\mathbf{t}_{a b}^{p, q}$ in Eq. A.5 for the even states, and in Sec. A.2.2 for the odd states.

\section{A.2.1 Even states}

The even states are composed of orbitals of $E^{\prime}$ and $A_{1}^{\prime}$ symmetry,

$$
\begin{aligned}
& d_{3 z^{2}-r^{2}}=|0\rangle, d_{x y}=|1\rangle, d_{x^{2}-y^{2}}=|2\rangle \\
& \frac{1}{\sqrt{2}}\left[p_{x}\left(\mathrm{X}_{1}\right)+p_{x}\left(\mathrm{X}_{2}\right)\right]=|3\rangle, \frac{1}{\sqrt{2}}\left[p_{y}\left(\mathrm{X}_{1}\right)+p_{y}\left(\mathrm{X}_{2}\right)\right]=|4\rangle \\
& \frac{1}{\sqrt{2}}\left[p_{z}\left(\mathrm{X}_{1}\right)-p_{z}\left(\mathrm{X}_{2}\right)\right]=|5\rangle
\end{aligned}
$$

The matrix blocks $\epsilon_{a}$ and $\mathbf{t}_{a b}^{p, q}$ in Eq. A.5 are then all $3 \times 3$

$$
\boldsymbol{\epsilon}_{d}=\left[\begin{array}{ccc}
\epsilon_{3 z^{2}-r^{2}} & 0 & 0 \\
0 & \epsilon_{x y} & 0 \\
0 & 0 & \epsilon_{x y}
\end{array}\right], \boldsymbol{\epsilon}_{p}=\left[\begin{array}{ccc}
\epsilon_{p_{x}}+V_{p p \pi} & 0 & 0 \\
0 & \epsilon_{p_{x}}+V_{p p \pi} & 0 \\
0 & 0 & \epsilon_{p_{z}}-V_{p p \sigma}
\end{array}\right]
$$

where $\epsilon_{a}$ are the on-site orbital energies and $V_{a b \alpha}$ are the Slater-Koster two-center integrals [226]. Similarly,

$$
\begin{gathered}
\mathbf{t}_{d p}^{0,0}=\frac{\sqrt{2}}{7 \sqrt{7}}\left[\begin{array}{rrr}
9 V_{p d \pi}-\sqrt{3} V_{p d \sigma} & 3 \sqrt{3} V_{p d \pi}-V_{p d \sigma} & 12 V_{p d \pi}+\sqrt{3} V_{p d \sigma} \\
-V_{p d \pi}-3 \sqrt{3} V_{p d \sigma} & -5 \sqrt{3} V_{p d \pi}-3 V_{p d \sigma} & -6 V_{p d \pi}+3 \sqrt{3} V_{p d \sigma} \\
-5 \sqrt{3} V_{p d \pi}-3 V_{p d \sigma} & 9 V_{p d \pi}-\sqrt{3} V_{p d \sigma} & -2 \sqrt{3} V_{p d \pi}+3 V_{p d \sigma}
\end{array}\right], \\
\mathbf{t}_{d d}^{1,0}=\left[\begin{array}{ccc}
\frac{1}{4}\left(3 V_{d d \delta}+V_{d d \sigma}\right) & \frac{3}{8}\left(-V_{d d \delta}+V_{d d \sigma}\right) & \frac{\sqrt{3}}{8}\left(-V_{d d \delta}+V_{d d \sigma}\right) \\
\frac{3}{8}\left(-V_{d d \delta}+V_{d d \sigma}\right) & \frac{1}{16}\left(3 V_{d d \delta}+4 V_{p p \pi}+9 V_{d d \sigma}\right) & \frac{\sqrt{3}}{16}\left(V_{d d \delta}-4 V_{d d \pi}+3 V_{d d \sigma}\right) \\
\frac{\sqrt{3}}{8}\left(-V_{d d \delta}+V_{d d \sigma}\right) & \frac{\sqrt{3}}{16}\left(V_{d d \delta}-4 V_{d d \pi}+3 V_{d d \sigma}\right) & \frac{1}{16}\left(V_{d d \delta}+12 V_{p p \pi}+3 V_{d d \sigma}\right)
\end{array}\right], \\
\mathbf{t}_{p p}^{1,0}=\left[\begin{array}{rrr}
\frac{1}{4}\left(3 V_{p p \pi}+V_{p p \sigma}\right) & \frac{\sqrt{3}}{4}\left(V_{p p \pi}-V_{p p \sigma}\right) & 0 \\
\frac{\sqrt{3}}{4}\left(V_{p p \pi}-V_{p p \sigma}\right) & \frac{1}{4}\left(V_{p p \pi}+3 V_{p p \sigma}\right) & 0 \\
0 & 0 & V_{p p \pi}
\end{array}\right],
\end{gathered}
$$




$$
\begin{aligned}
& \mathbf{t}_{d d}^{0,1}=\left[\begin{array}{ccc}
\frac{1}{4}\left(3 V_{d d \delta}+V_{d d \sigma}\right) & \frac{3}{8}\left(V_{d d \delta}-V_{d d \sigma}\right) & \frac{\sqrt{3}}{8}\left(-V_{d d \delta}+V_{d d \sigma}\right) \\
\frac{3}{8}\left(V_{d d \delta}-V_{d d \sigma}\right) & \frac{1}{16}\left(3 V_{d d \delta}+4 V_{p p \pi}+9 V_{d d \sigma}\right) & \frac{\sqrt{3}}{16}\left(-V_{d d \delta}+4 V_{d d \pi}-3 V_{d d \sigma}\right) \\
\frac{\sqrt{3}}{8}\left(-V_{d d \delta}+V_{d d \sigma}\right) & \frac{\sqrt{3}}{16}\left(-V_{d d \delta}+4 V_{d d \pi}-3 V_{d d \sigma}\right) & \frac{1}{16}\left(V_{d d \delta}+12 V_{p p \pi}+3 V_{d d \sigma}\right)
\end{array}\right], \\
& \mathbf{t}_{p p}^{0,1}=\left[\begin{array}{ccc}
\frac{1}{4}\left(3 V_{p p \pi}+V_{p p \sigma}\right) & \frac{\sqrt{3}}{4}\left(-V_{p p \pi}+V_{p p \sigma}\right) & 0 \\
\frac{\sqrt{3}}{4}\left(-V_{p p \pi}+V_{p p \sigma}\right) & \frac{1}{4}\left(V_{p p \pi}+3 V_{p p \sigma}\right) & 0 \\
0 & 0 & V_{p p \pi}
\end{array}\right], \\
& \mathbf{t}_{d p}^{0,1}=\frac{2 \sqrt{2}}{7 \sqrt{7}}\left[\begin{array}{ccc}
0 & -3 \sqrt{3} V_{p d \pi}+V_{p d \sigma} & 6 V_{p d \pi}+\frac{1}{2} \sqrt{3} V_{p d \sigma} \\
V_{p d \pi} & 0 & 0 \\
0 & -3 V_{p d \pi}-2 \sqrt{3} V_{p d \sigma} & 2 \sqrt{3} V_{p d \pi}-3 V_{p d \sigma}
\end{array}\right], \\
& \mathbf{t}_{d d}^{1,1}=\left[\begin{array}{ccc}
\frac{1}{4}\left(3 V_{d d \delta}+V_{d d \sigma}\right) & 0 & \frac{\sqrt{3}}{4}\left(V_{d d \delta}-V_{d d \sigma}\right) \\
0 & V_{d d \pi} & 0 \\
\frac{\sqrt{3}}{4}\left(V_{d d \delta}-V_{d d \sigma}\right) & 0 & \frac{1}{4}\left(V_{d d \delta}+3 V_{d d \sigma}\right)
\end{array}\right], \quad \mathbf{t}_{p p}^{1,1}=\left[\begin{array}{ccc}
V_{p p \sigma} & 0 & 0 \\
0 & V_{p p \pi} & 0 \\
0 & 0 & V_{p p \pi}
\end{array}\right], \\
& \mathbf{t}_{d p}^{1,1}=\frac{\sqrt{2}}{7 \sqrt{7}}\left[\begin{array}{ccc}
-9 V_{p d \pi}+\sqrt{3} V_{p d \sigma} & 3 \sqrt{3} V_{p d \pi}-V_{p d \sigma} & 12 V_{p d \pi}+\sqrt{3} V_{p d \sigma} \\
-V_{p d \pi}-3 \sqrt{3} V_{p d \sigma} & 5 \sqrt{3} V_{p d \pi}+3 V_{p d \sigma} & 6 V_{p d \pi}-3 \sqrt{3} V_{p d \sigma} \\
5 \sqrt{3} V_{p d \pi}+3 V_{p d \sigma} & 9 V_{p d \pi}-\sqrt{3} V_{p d \sigma} & -2 \sqrt{3} V_{p d \pi}+3 V_{p d \sigma}
\end{array}\right] \text {. }
\end{aligned}
$$

We use values of the parameters as obtained by Rostami at al. from fitting the even tight-binding bands to DFT GGA/PBE bands [205]. A lattice parameter of $3.16 \AA$ for $\mathrm{MoS}_{2}$ has been used in these calculations, which is $1 \%$ smaller than the optimized GGA/PBE value, but close to the reported experimental values. It results in a calculated band gap of $1.76 \mathrm{eV}$, which is slightly smaller than the experimental optical band gap of $1.85 \mathrm{eV}$ [25]. We have shifted the tight-binding bands such, that the zero of energy coincides with the top of the valence band.

\section{A.2.2 Odd states}

The odd states are composed of orbitals of $E^{\prime \prime}$ and $A_{1}^{\prime \prime}$ symmetry,

$$
\begin{aligned}
& d_{x z}=\left|1^{\prime}\right\rangle, d_{y z}=\left|2^{\prime}\right\rangle, \\
& \frac{1}{\sqrt{2}}\left[p_{x}\left(\mathrm{X}_{1}\right)-p_{x}\left(\mathrm{X}_{2}\right)\right]=\left|3^{\prime}\right\rangle, \frac{1}{\sqrt{2}}\left[p_{y}\left(\mathrm{X}_{1}\right)-p_{y}\left(\mathrm{X}_{2}\right)\right]=\left|4^{\prime}\right\rangle \\
& \frac{1}{\sqrt{2}}\left[p_{z}\left(\mathrm{X}_{1}\right)+p_{z}\left(\mathrm{X}_{2}\right)\right]=\left|5^{\prime}\right\rangle .
\end{aligned}
$$

The matrix blocks $\epsilon_{a}$ in Eq. A.5 are $2 \times 2$ if $a=d$ and $3 \times 3$ if $a=p$, whereas the matrix blocks $\mathbf{t}_{a b}^{p, q}$ are $2 \times 2$ if $a b=d d, 3 \times 3$ if $a b=p p$, and $2 \times 3$ if $a b=d p$

$$
\boldsymbol{\epsilon}_{d}=\left[\begin{array}{cc}
\epsilon_{x z}^{\prime} & 0 \\
0 & \epsilon_{x z}^{\prime}
\end{array}\right], \boldsymbol{\epsilon}_{p}=\left[\begin{array}{ccc}
\epsilon_{p_{x}}^{\prime}-V_{p p \pi}^{\prime} & 0 & 0 \\
0 & \epsilon_{p_{x}}^{\prime}-V_{p p \pi}^{\prime} & 0 \\
0 & 0 & \epsilon_{p_{z}}^{\prime}+V_{p p \sigma}^{\prime}
\end{array}\right]
$$




$$
\begin{aligned}
& \mathbf{t}_{d p}^{0,0}=\frac{\sqrt{2}}{7 \sqrt{7}}\left[\begin{array}{ccc}
\sqrt{3} V_{p d \pi}^{\prime}+9 V_{p d \sigma}^{\prime} & -6 V_{p d \pi}^{\prime}+3 \sqrt{3} V_{p d \sigma}^{\prime} & -\sqrt{3} V_{p d \pi}^{\prime}-9 V_{p d \sigma}^{\prime} \\
-6 V_{p d \pi}^{\prime}+3 \sqrt{3} V_{p d \sigma}^{\prime} & 5 \sqrt{3} V_{p d \pi}^{\prime}+3 V_{p d \sigma}^{\prime} & -V_{p d \pi}^{\prime}-3 \sqrt{3} V_{p d \sigma}^{\prime}
\end{array}\right], \\
& \mathbf{t}_{d d}^{1,0}=\left[\begin{array}{cc}
\frac{1}{4}\left(3 V_{d d \delta}^{\prime}+V_{d d \pi}^{\prime}\right) & \frac{\sqrt{3}}{4}\left(V_{d d \delta}^{\prime}-V_{d d \pi}^{\prime}\right) \\
\frac{\sqrt{3}}{4}\left(V_{d d \delta}^{\prime}-V_{d d \pi}^{\prime}\right) & \frac{1}{4}\left(V_{d d \delta}^{\prime}+3 V_{p p \pi)}^{\prime}\right.
\end{array}\right] \\
& \mathbf{t}_{p p}^{1,0}=\left[\begin{array}{ccc}
\frac{1}{4}\left(3 V_{p p \pi}^{\prime}+V_{p p \sigma}^{\prime}\right) & \frac{\sqrt{3}}{4}\left(V_{p p \pi}^{\prime}-V_{p p \sigma}^{\prime}\right) & 0 \\
\frac{\sqrt{3}}{4}\left(V_{p p \pi}^{\prime}-V_{p p \sigma}^{\prime}\right) & \frac{1}{4}\left(V_{p p \pi}^{\prime}+3 V_{p p \sigma}^{\prime}\right) & 0 \\
0 & 0 & V_{p p \pi}^{\prime}
\end{array}\right] \text {, } \\
& \mathbf{t}_{d d}^{0,1}=\left[\begin{array}{cc}
\frac{1}{4}\left(3 V_{d d \delta}^{\prime}+V_{d d \pi}^{\prime}\right) & \frac{\sqrt{3}}{4}\left(-V_{d d \delta}^{\prime}+V_{d d \pi}^{\prime}\right) \\
\frac{\sqrt{3}}{4}\left(-V_{d d \delta}^{\prime}+V_{d d \pi}^{\prime}\right) & \frac{1}{4}\left(V_{d d \delta}^{\prime}+3 V_{d d \pi}^{\prime}\right)
\end{array}\right] \\
& \mathbf{t}_{p p}^{0,1}=\left[\begin{array}{ccc}
\frac{1}{4}\left(3 V_{p p \pi}^{\prime}+V_{p p \sigma}^{\prime}\right) & \frac{\sqrt{3}}{4}\left(-V_{p p \pi}^{\prime}+V_{p p \sigma}^{\prime}\right) & 0 \\
\frac{\sqrt{3}}{4}\left(-V_{p p \pi}^{\prime}+V_{p p \sigma}^{\prime}\right) & \frac{1}{4}\left(V_{p p \pi}^{\prime}+3 V_{p p \sigma}^{\prime}\right) & 0 \\
0 & 0 & V_{p p \pi}^{\prime}
\end{array}\right] \text {, } \\
& \mathbf{t}_{d p}^{0,1}=\frac{\sqrt{2}}{7 \sqrt{7}}\left[\begin{array}{ccc}
\sqrt{3} V_{p d \pi}^{\prime} & 0 & 0 \\
0 & -\sqrt{3} V_{p d \pi}^{\prime}+12 V_{p d \sigma}^{\prime} & 2 V_{p d \pi}^{\prime}+6 \sqrt{3} V_{p d \sigma}^{\prime}
\end{array}\right] \\
& \mathbf{t}_{d d}^{1,1}=\left[\begin{array}{cc}
V_{d d \pi}^{\prime} & 0 \\
0 & V_{d d \delta}^{\prime}
\end{array}\right], \mathbf{t}_{p p}^{1,1}=\left[\begin{array}{ccc}
V_{p p \sigma}^{\prime} & 0 & 0 \\
0 & V_{p p \pi}^{\prime} & 0 \\
0 & 0 & V_{p p \pi}^{\prime}
\end{array}\right] \\
& \mathbf{t}_{d p}^{1,1}=\frac{\sqrt{2}}{7 \sqrt{7}}\left[\begin{array}{ccc}
\sqrt{3} V_{p d \pi}^{\prime}+9 V_{p d \sigma}^{\prime} & 6 V_{p d \pi}^{\prime}-3 \sqrt{3} V_{p d \sigma}^{\prime} & \sqrt{3} V_{p d \pi}^{\prime}+9 V_{p d \sigma}^{\prime} \\
6 V_{p d \pi}^{\prime}-3 \sqrt{3} V_{p d \sigma}^{\prime} & 5 \sqrt{3} V_{p d \pi}^{\prime}+3 V_{p d \sigma}^{\prime} & -V_{p d \pi}^{\prime}-3 \sqrt{3} V_{p d \sigma}^{\prime}
\end{array}\right] .
\end{aligned}
$$

We obtain values of the parameters by fitting the tight-binding bands of odd symmetry to bands obtained from a density functional theory (DFT) calculation with the generalized gradient GGA/PBE functional [59, 46, 47], using the same lattice parameter as for the even bands. The optimal parameters are given in table A.1, and the quality of the fit can be judged from Fig. A.1. 
Table A.1: Values of the tight-binding parameters $(\mathrm{eV})$ used for the bands of odd symmetry.

\begin{tabular}{|c|c||c|c|}
\hline$\epsilon_{p_{x}}^{\prime}$ & -2.188 & $\epsilon_{p_{z}}^{\prime}$ & -0.682 \\
$\epsilon_{x z}^{\prime}$ & 0.604 & & \\
$V_{d d \pi}^{\prime}$ & -0.075 & $V_{d d \delta}^{\prime}$ & 0.051 \\
$V_{p p \sigma}^{\prime}$ & 1.166 & $V_{p p \pi}^{\prime}$ & -0.389 \\
$V_{p d \sigma}^{\prime}$ & 2.115 & $V_{p d \pi}^{\prime}$ & -0.626 \\
\hline
\end{tabular}

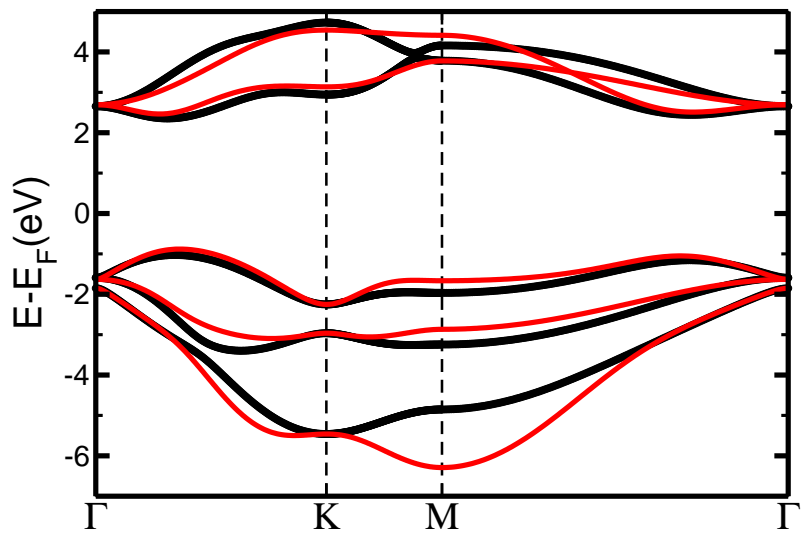

Figure A.1: (Color online) GGA/PBE band structure (black) of the odd symmetry bands of $\mathrm{MoS}_{2}$; (red) tight-binding fit. 


\section{References}

[1] S. J. Kim, K. Choi, B. Lee, Y. Kim, and B. H. Hong, "Materials for Flexible, Stretchable Electronics: Graphene and 2D Materials," Annu. Rev. Mater. Res. 45, 63-84 (2015).

[2] D. Akinwande, N. Petrone, and J. Hone, "Two-dimensional flexible nanoelectronics," Nat. Commun. 5, 5678 (2014).

[3] H. Wang, F. Liu, W. Fu, Z. Fang, W. Zhou, and Z. Liu, "Two-dimensional heterostructures: fabrication, characterization, and application," Nanoscale 6, 12250-12272 (2014).

[4] M. Xu, T. Liang, M. Shi, and H. Chen, "Graphene-like two-dimensional materials," Chem. Rev. 113, 3766-3798 (2013).

[5] S. Z. Butler, S. M. Hollen, L. Cao, Y. Cui, J. A. Gupta, H. R. Gutirrez, T. F. Heinz, S. S. Hong, J. Huang, A. F. Ismach, E. Johnston-Halperin, M. Kuno, V. V. Plashnitsa, R. D. Robinson, R. S. Ruoff, S. Salahuddin, J. Shan, L. Shi, M. G. Spencer, M. Terrones, W. Windl, and J. E. Goldberger, "Progress, Challenges, and Opportunities in Two-Dimensional Materials Beyond Graphene," ACS Nano 7, 2898-2926 (2013).

[6] S. Das, J. A. Robinson, M. Dubey, H. Terrones, and M. Terrones, "Beyond Graphene: Progress in Novel Two-Dimensional Materials and van der Waals Solids," Annu. Rev. Mater. Res. 45, 1-27 (2015).

[7] G. R. Bhimanapati, Z. Lin, V. Meunier, Y. Jung, J. Cha, S. Das, D. Xiao, Y. Son, M. S. Strano, V. R. Cooper, L. Liang, S. G. Louie, E. Ringe, W. Zhou, S. S. Kim, R. R. Naik, B. G. Sumpter, H. Terrones, F. Xia, Y. Wang, J. Zhu, D. Akinwande, N. Alem, J. A. Schuller, R. E. Schaak, M. Terrones, and J. A. Robinson, "Recent Advances in Two-Dimensional Materials beyond Graphene," ACS Nano 9, 11509-11539 (2015).

[8] S. Balendhran, S. Walia, H. Nili, S. Sriram, and M. Bhaskaran, "Elemental analogues of graphene: Silicene, germanene, stanene, and phosphorene," Small 11, 640-652 (2015). 
[9] A. Dimoulas, "Silicene and germanene: Silicon and germanium in the flatland," Microelectron. Eng. 131, 68-78 (2015).

[10] A. Acun, L. Zhang, P. Bampoulis, M. Farmanbar, A. van Houselt, A. N. Rudenko, M. Lingenfelder, G. Brocks, B. Poelsema, M. I. Katsnelson, and H. J. W. Zandvliet, "Germanene: the germanium analogue of graphene," J. Phys.: Condens. Matter 27, 443002 (2015).

[11] X. Ling, H. Wang, S. Huang, F. Xia, and M. S. Dresselhaus, "The renaissance of black phosphorus," Proc. Natl. Acad. Sci. U.S.A. 112, 4523-4530 (2015).

[12] G.-B. Liu, D. Xiao, Y. Yao, X. Xu, and W. Yao, “Electronic structures and theoretical modelling of two-dimensional group-VIB transition metal dichalcogenides," Chem. Soc. Rev. 44, 2643-2663 (2015).

[13] S. Cahangirov, M. Topsakal, E. Aktürk, H. Şahin, and S. Ciraci, "Two- and one-dimensional honeycomb structures of silicon and germanium," Phys. Rev. Lett. 102, 236804 (2009).

[14] J. Kim, S. S. Baik, S. H. Ryu, Y. Sohn, S. Park, B.-G. Park, J. Denlinger, Y. Yi, H. J. Choi, and K. S. Kim, "Observation of tunable band gap and anisotropic dirac semimetal state in black phosphorus," Science 349, 723-726 (2015).

[15] L. Li, Y. Yu, G. J. Ye, Q. Ge, X. Ou, H. Wu, D. Feng, X. H. Chen, and Y. Zhang, "Black phosphorus field-effect transistors," Nature Nanotech. 9, 372-377 (2014).

[16] H. Liu, A. T. Neal, Z. Zhu, Z. Luo, X. Xu, D. Tománek, and P. D. Ye, "Phosphorene: An Unexplored 2D Semiconductor with a High Hole Mobility," ACS Nano 8, 4033-4041 (2014).

[17] L. C. Gomes and A. Carvalho, "Phosphorene analogues: Isoelectronic twodimensional group-IV monochalcogenides with orthorhombic structure," Phys. Rev. B 92, 085406 (2015).

[18] K. Watanabe, T. Taniguchi, and H. Kanda, "Direct-bandgap properties and evidence for ultraviolet lasing of hexagonal boron nitride single crystal," Nature Mater. 3, 404-409 (2004).

[19] C. R. Dean, A. F. Young, I. Meric, C. Lee, L. Wang, S. Sorgenfrei, K. Watanabe, T. Taniguchi, P. Kim, K. L. Shepard, and J. Hone, "Boron nitride substrates for high-quality graphene electronics," Nature Nanotech. 5, 722-726 (2010).

[20] Y. Kubota, K. Watanabe, O. Tsuda, and T. Taniguchi, "Deep ultraviolet lightemitting hexagonal boron nitride synthesized at atmospheric pressure," Science 317, 932-934 (2007). 
[21] M. Chhowalla, H. S. Shin, G. Eda, L.-J. Li, K. P. Loh, and H. Zhang, "The chemistry of two-dimensional layered transition metal dichalcogenide nanosheets," Nat. Chem. 5, 263275 (2013).

[22] D. Jariwala, V. K. Sangwan, L. J. Lauhon, T. J. Marks, and M. C. Hersam, "Emerging device applications for semiconducting two-dimensional transition metal dichalcogenides," ACS Nano 8, 1102-1120 (2014).

[23] R. Ganatra and Q. Zhang, "Few-Layer $\mathrm{MoS}_{2}$ : A Promising Layered Semiconductor," ACS Nano 8, 4074-4099 (2014).

[24] Y. Huang, J. Guo, Y. Kang, Y. Ai, and C. M. Li, "Two dimensional atomically thin $\mathrm{MoS}_{2}$ nanosheets and their sensing applications," Nanoscale 7, 1935819376 (2015).

[25] K. F. Mak, C. Lee, J. Hone, J. Shan, and T. F. Heinz, "Atomically thin $\mathrm{MoS}_{2}$ : A new direct-gap semiconductor," Phys. Rev. Lett. 105, 136805 (2010).

[26] D. Tománek, "Interfacing graphene and related 2D materials with the 3D world," J. Phys.: Condens. Matter 27, 133203 (2015).

[27] L. Brillson, "The structure and properties of metal-semiconductor interfaces," Surf. Sci. Rep. 2, 123 - 326 (1982).

[28] R. T. Tung, "The physics and chemistry of the schottky barrier height," Appl. Phys. Rep. 1, 011304 (2014).

[29] J. Tersoff, "Schottky barriers and semiconductor band structures," Phys. Rev. B 32, 6968-6971 (1985).

[30] R. T. Tung, "Recent advances in schottky barrier concepts," Mat. Sci. Eng. R. $35,1-138$ (2001).

[31] W. Mönch, "Metal-semiconductor contacts: electronic properties," Surf. Sci. 299, 928 - 944 (1994).

[32] H. Hasegawa, "Fermi Level Pinning and Schottky Barrier Height Control at Metal-Semiconductor Interfaces of InP and Related Materials," Jpn. J. Appl. Phys. 38, 1098 (1999).

[33] S. G. Louie, J. R. Chelikowsky, and M. L. Cohen, "Ionicity and the theory of schottky barriers," Phys. Rev. B 15, 2154-2162 (1977).

[34] W. Mönch, "On the physics of metal-semiconductor interfaces," Rep. Prog. Phys 53, 221 (1990).

[35] O. F. Sankey, R. E. Allen, S.-. Ren, and J. D. Dow, “Dangling bonds and schottky barriers," J. Vac. Sci. Technol. B 3, 1162-1166 (1985). 
[36] V. Heine, “Theory of surface states," Phys. Rev. 138, A1689-A1696 (1965).

[37] T. Nishimura, K. Kita, and A. Toriumi, "Evidence for strong fermi-level pinning due to metal-induced gap states at metal/germanium interface," Appl. Phys. Lett. 91, 123123 (2007).

[38] A. Dimoulas, P. Tsipas, A. Sotiropoulos, and E. K. Evangelou, "Fermi-level pinning and charge neutrality level in germanium," Appl. Phys. Lett. 89, 252110 (2006).

[39] J. Tersoff, "Schottky barrier heights and the continuum of gap states," Phys. Rev. Lett. 52, 465-468 (1984).

[40] M. Bokdam, G. Brocks, and P. J. Kelly, "Large potential steps at weakly interacting metal-insulator interfaces," Phys. Rev. B 90, 201411 (2014).

[41] F. Güller, A. M. Llois, J. Goniakowski, and C. Noguera, "Polarity effects in unsupported polar nanoribbons," Phys. Rev. B 87, 205423 (2013).

[42] J. Wu, W. Pisula, , and K. Müllen, "Graphenes as potential material for electronics," Chem. Rev. 107, 718-747 (2007).

[43] S. Dutta and S. K. Pati, "Novel properties of graphene nanoribbons: a review," J. Mater. Chem. 20, 8207-8223 (2010).

[44] G. Kresse and J. Hafner, "Ab-initio molecular-dynamics for liquid-metals," Phys. Rev. B 47, 558-561 (1993).

[45] P. E. Blöchl, "Projector augmented-wave method," Phys. Rev. B 50, 17953 17979 (1994).

[46] G. Kresse and J. Furthmüller, "Efficient iterative schemes for ab initio totalenergy calculations using a plane-wave basis set," Phys. Rev. B 54, 11169-11186 (1996).

[47] G. Kresse and D. Joubert, "From ultrasoft pseudopotentials to the projector augmented-wave method," Phys. Rev. B 59, 1758-1775 (1999).

[48] P. Hohenberg and W. Kohn, "Inhomogeneous electron gas," Phys. Rev. 136, B864-B871 (1964).

[49] W. Kohn and L. J. Sham, "Self-consistent equations including exchange and correlation effects," Phys. Rev. 140, A1133-A1138 (1965).

[50] R. O. Jones and O. Gunnarsson, "The density functional formalism, its applications and prospects," Rev. Mod. Phys. 61, 689-746 (1989).

[51] W. Kohn, "Nobel lecture: Electronic structure of matter-wave functions and density functionals," Rev. Mod. Phys. 71, 1253-1266 (1999). 
[52] N. Argaman and G. Makov, "Density functional theory: An introduction," Am. J. Phys. 68, 69-79 (2000).

[53] J. Kohanoff, Electronic Structure Calculations for Solids and Molecules: Theory and Computational Methods (Cambridge University Press, 2006).

[54] R. M. Martin, Electronic Structure: Basic Theory and Practical Methods (Cambridge University Press, 2008).

[55] J. P. Perdew and A. Zunger, "Self-interaction correction to density-functional approximations for many-electron systems," Phys. Rev. B 23, 5048-5079 (1981).

[56] D. M. Ceperley and B. J. Alder, "Ground state of the electron gas by a stochastic method," Phys. Rev. Lett. 45, 566-569 (1980).

[57] M. T. Yin and M. L. Cohen, "Structural theory of graphite and graphitic silicon," Phys. Rev. B 29, 6996-6998 (1984).

[58] T. Björkman, A. Gulans, A. V. Krasheninnikov, and R. M. Nieminen, “Are we van der waals ready?" J. Phys.: Condens. Matter 24, 424218 (2012).

[59] J. P. Perdew, K. Burke, and M. Ernzerhof, "Generalized gradient approximation made simple," Phys. Rev. Lett. 77, 3865-3868 (1996).

[60] E. Hazrati, G. A. de Wijs, and G. Brocks, "Li intercalation in graphite: A van der waals density-functional study," Phys. Rev. B 90, 155448 (2014).

[61] H. Peelaers and C. V. de Walle, "First-principles study of van der Waals interactions in $\mathrm{MoS}_{2}$ and $\mathrm{MoO}_{3}$," J. Phys.: Condens. Matter 26, 305502 (2014).

[62] J. Harris, "Simplified method for calculating the energy of weakly interacting fragments," Phys. Rev. B 31, 1770-1779 (1985).

[63] M. Dion, H. Rydberg, E. Schröder, D. C. Langreth, and B. I. Lundqvist, "Van der waals density functional for general geometries," Phys. Rev. Lett. 92, 246401 (2004).

[64] J. Klimeš, D. R. Bowler, and A. Michaelides, "Van der waals density functionals applied to solids," Phys. Rev. B 83, 195131 (2011).

[65] T. Thonhauser, V. R. Cooper, S. Li, A. Puzder, P. Hyldgaard, and D. C. Langreth, "Van der waals density functional: Self-consistent potential and the nature of the van der waals bond," Phys. Rev. B 76, 125112 (2007).

[66] K. Lee, E. D. Murray, L. Kong, B. I. Lundqvist, and D. C. Langreth, "Higheraccuracy van der waals density functional," Phys. Rev. B 82, 081101 (2010).

[67] J. Klimeš, D. R. Bowler, and A. Michaelides, "Chemical accuracy for the van der waals density functional," J. Phys.: Condens. Matter 22, 022201 (2010). 
[68] A. K. Geim and I. V. Grigorieva, "Van der waals heterostructures," Nature 499, 419-425 (2013).

[69] Q. H. Wang, K. Kalantar-Zadeh, A. Kis, J. N. Coleman, and M. S. Strano, "Electronics and optoelectronics of two-dimensional transition metal dichalcogenides," Nat. Nanotechnol. 7, 699-712 (2012).

[70] D. Lembke, S. Bertolazzi, and A. Kis, "Single-layer $\mathrm{MoS}_{2}$ electronics," Accounts Chem. Res. 48, 100-110 (2015).

[71] H. Liu, A. T. Neal, and P. D. Ye, "Channel length scaling of MoS 2 MOSFETs," ACS Nano 10, 8563-8569 (2012).

[72] S. Das, H.-Y. Chen, A. V. Penumatcha, and J. Appenzeller, "High performance multilayer $\mathrm{MoS}_{2}$ transistors with scandium contacts," Nano Lett. 13, 100-105 (2013).

[73] J.-R. Chen, P. M. Odenthal, A. G. Swartz, G. C. Floyd, K. Y. L. Hua Wen, and R. K. Kawakami, "Control of schottky barriers in single layer $\mathrm{MoS}_{2}$ transistors with ferromagnetic contacts," Nano Lett. 13, 3106-3110 (2013).

[74] M. Fontana, T. Deppe, A. K. Boyd, M. Rinzan, A. Y. Liu, M. Paranjape, and P. Barbara, "Electron-hole transport and photovoltaic effect in gated $\mathrm{MoS}_{2}$ schottky junctions," Sci. Rep. 3, 113505 (2013).

[75] N. Kaushik, A. Nipane, F. Basheer, S. Dubey, S. Grover, M. M. Deshmukh, and S. Lodha, "Schottky barrier heights for $\mathrm{Au}$ and $\mathrm{Pd}$ contacts to $\mathrm{MoS}_{2}$," Appl. Phys. Lett. 105, 113505 (2014).

[76] J. Kang, W. Liu, and K. Banerjee, "High-performance $\mathrm{MoS}_{2}$ transistors with low-resistance molybdenum contacts," Appl. Phys. Lett. 104, 093106 (2014).

[77] M. Farmanbar and G. Brocks, "Controlling the schottky barrier at $\mathrm{MoS}_{2} /$ metal contacts by inserting a bn monolayer," Phys. Rev. B 91, 161304 (2015).

[78] M. Bokdam, G. Brocks, M. I. Katsnelson, and P. J. Kelly, "Schottky barriers at hexagonal boron nitride/metal interfaces: A first-principles study," Phys. Rev. B 90, 085415 (2014).

[79] C. Ataca, H. Şahin, and S. Ciraci, "Stable, single-layer $\mathrm{MX}_{2}$ transition-metal oxides and dichalcogenides in a honeycomb-like structure," J. Phys. Chem. C 116, 8983-8999 (2012).

[80] W. A. Saidi, "Influence of strain and metal thickness on metal-MoS ${ }_{2}$ contacts," J. Chem. Phys. 141, 094707 (2014).

[81] I. Popov, G. Seifert, and D. Tománek, "Designing electrical contacts to $\mathrm{MoS}_{2}$ monolayers: A computational study," Phys. Rev. Lett. 108, 156802 (2012). 
[82] W. Chen, E. J. G. Santos, W. Zhu, E. Kaxiras, and Z. Zhang, "Tuning the electronic and chemical properties of monolayer $\mathrm{MoS}_{2}$ adsorbed on transition metal substrates," Nano Lett. 13, 509-514 (2013).

[83] J. Kang, W. Liu, D. Sarkar, D. Jena, and K. Banerjee, “Computational study of metal contacts to monolayer transition-metal dichalcogenide semiconductors," Phys. Rev. X 4, 031005 (2014).

[84] C. Gong, L. Colombo, R. M. Wallace, and K. Cho, "The unusual mechanism of partial fermi level pinning at metal/ $\mathrm{MoS}_{2}$ interfaces," Nano Lett. 14, 17141720 (2014).

[85] Z. Li, X. Li, and J. Yang, "Comparative study on electronic structures of Sc and Ti contacts with monolayer and multilayer $\mathrm{MoS}_{2}, "$ ACS Appl. Mater. Interfaces 7, 12981-12987 (2015).

[86] G. Giovannetti, P. A. Khomyakov, G. Brocks, V. M. Karpan, J. van den Brink, and P. J. Kelly, "Doping graphene with metal contacts," Phys. Rev. Lett. 101, 026803 (2008).

[87] R. Laskowski, P. Blaha, and K. Schwarz, "Bonding of hexagonal bn to transition metal surfaces: An ab initio density-functional theory study," Phys. Rev. B 78, 045409 (2008).

[88] P. A. Khomyakov, G. Giovannetti, P. C. Rusu, G. Brocks, J. van den Brink, and P. J. Kelly, "First-principles study of the interaction and charge transfer between graphene and metals," Phys. Rev. B 79, 195425 (2009).

[89] D. Stradi, S. Barja, C. Díaz, M. Garnica, B. Borca, J. J. Hinarejos, D. SánchezPortal, M. Alcamí, A. Arnau, A. L. Vázquez de Parga, R. Miranda, and F. Martín, "Role of dispersion forces in the structure of graphene monolayers on Ru surfaces," Phys. Rev. Lett. 106, 186102 (2011).

[90] T. Olsen, J. Yan, J. J. Mortensen, and K. S. Thygesen, “Dispersive and covalent interactions between graphene and metal surfaces from the random phase approximation," Phys. Rev. Lett. 107, 156401 (2011).

[91] M. Andersen, L. Hornekær, and B. Hammer, "Graphene on metal surfaces and its hydrogen adsorption: A meta-GGA functional study," Phys. Rev. B 86, 085405 (2012).

[92] P. Janthon, F. Viñes, S. M. Kozlov, J. Limtrakul, and F. Illas, “Theoretical assessment of graphene-metal contacts," J. Chem. Phys. 138, 244701 (2013).

[93] D. Çakır, C. Sevik, and F. M. Peeters, "Engineering electronic properties of metal-MoSe $e_{2}$ interfaces using self-assembled monolayers," J. Mater. Chem. C 2, 9842-9849 (2014). 
[94] D. Çakır and F. M. Peeters, "Dependence of the electronic and transport properties of metal-MoSe 2 interfaces on contact structures," Phys. Rev. B 89, 245403 (2014).

[95] B. Sachs, T. O. Wehling, M. I. Katsnelson, and A. I. Lichtenstein, "Adhesion and electronic structure of graphene on hexagonal boron nitride substrates," Phys. Rev. B 84, 195414 (2011).

[96] M. Methfessel and A. T. Paxton, "High-precision sampling for brillouin-zone integration in metals," Phys. Rev. B 40, 3616-3621 (1989).

[97] J. Neugebauer and M. Scheffler, "Adsorbate-substrate and adsorbateadsorbate interactions of $\mathrm{Na}$ and $\mathrm{K}$ adlayers on $\mathrm{Al}(111)$," Phys. Rev. B 46, 16067-16080 (1992).

[98] G. Giovannetti, P. A. Khomyakov, G. Brocks, P. J. Kelly, and J. van den Brink, "Substrate-induced band gap in graphene on hexagonal boron nitride: Ab initio density functional calculations," Phys. Rev. B 76, 073103 (2007).

[99] T. Björkman, A. Gulans, A. V. Krasheninnikov, and R. M. Nieminen, "van der waals bonding in layered compounds from advanced density-functional firstprinciples calculations," Phys. Rev. Lett. 108, 235502 (2012).

[100] I. Hamada, "van der waals density functional made accurate," Phys. Rev. B 89, 121103 (2014).

[101] H. Shi, H. Pan, Y.-W. Zhang, and B. Yakobson, "Quasiparticle band structures and optical properties of strained monolayer $\mathrm{MoS}_{2}$ and $\mathrm{WS}_{2}$," Phys. Rev. B 87, 155304 (2013).

[102] T. Li, "Ideal strength and phonon instability in single-layer $\mathrm{MoS}_{2}$," Phys. Rev. B 85, 235407 (2012).

[103] W. S. Yun, S. W. Han, S. C. Hong, I. G. Kim, and J. D. Lee, “Thickness and strain effects on electronic structures of transition metal dichalcogenides: $2 \mathrm{H}-\mathrm{MoS}_{2}$ semiconductors (M= Mo, W; X = S, Se, Te)," Phys. Rev. B 85, 033305 (2012).

[104] E. Cappelluti, R. Roldán, J. A. Silva-Guillén, P. Ordejón, and F. Guinea, “Tightbinding model and direct-gap/indirect-gap transition in single-layer and multilayer $\mathrm{MoS}_{2}, "$ Phys. Rev. B 88, 075409 (2013).

[105] F. Hüser, T. Olsen, and K. S. Thygesen, "How dielectric screening in twodimensional crystals affects the convergence of excited-state calculations: Monolayer MoS 2 ,' Phys. Rev. B 88, 245309 (2013).

[106] H. J. Conley, B. Wang, J. I. Ziegler, J. Richard F. Haglund, S. T. Pantelides, and K. I. Bolotin, "Bandgap engineering of strained monolayer and bilayer $\mathrm{MoS}_{2}$," Nano Lett. 13, 3626-3630 (2013). 
[107] J. R. Lince, D. J. Carré, and P. D. Fleischauer, "Schottky-barrier formation on a covalent semiconductor without fermi-level pinning: The metal- $\mathrm{MoS}_{2}(0001)$ interface," Phys. Rev. B 36, 1647-1656 (1987).

[108] C. Maurel, F. Ajustron, R. Pchou, G. Seine, and R. Coratger, "Electrical behavior of the $\mathrm{Au} / \mathrm{MoS}_{2}$ interface studied by light emission induced by scanning tunneling microscopy," Surf. Sci. 600, $442-447$ (2006).

[109] P. A. Young, "Lattice parameter measurements on molybdenum disulphide," J. Phys. D Appl. Phys. 1, 936 (1968).

[110] A. Al-Hilli and B. Evans, "The preparation and properties of transition metal dichalcogenide single crystals," J. Cryst. Growth 15, 93 - 101 (1972).

[111] T. Böker, R. Severin, A. Müller, C. Janowitz, R. Manzke, D. Voß, P. Krüger, A. Mazur, and J. Pollmann, "Band structure of $\mathrm{MoS}_{2}, \mathrm{MoSe}_{2}$, and $\mathrm{MoTe}_{2}$ : angle-resolved photoelectron spectroscopy and ab initio calculations," Phys. Rev. B 64, 235305 (2001).

[112] H.-P. Komsa and A. V. Krasheninnikov, "Electronic structures and optical properties of realistic transition metal dichalcogenide heterostructures from first principles," Phys. Rev. B 88, 085318 (2013).

[113] C. Gong, G. Lee, B. Shan, E. M. Vogel, R. M. Wallace, and K. Cho, "Firstprinciples study of metal/graphene interfaces," J. Appl. Phys. 108, 123711 (2010).

[114] S. Tongay, J. Zhou, C. Ataca, K. Lo, T. S. Matthews, J. Li, J. C. Grossman, and $\mathrm{J}$. Wu, "Thermally Driven Crossover from Indirect toward Direct Bandgap in 2D Semiconductors: $\mathrm{MoSe}_{2}$ versus $\mathrm{MoS}_{2}$," Nano Lett. 12, 5576-5580 (2012).

[115] M. M. Ugeda, A. J. Bradley, S.-F. Shi, F. H. da Jornada, Y. Zhang, D. Y. Qiu, W. Ruan, S.-K. Mo, Z. Hussain, Z.-X. Shen, F. Wang, S. G. Louie, and M. F. Crommie, "Giant bandgap renormalization and excitonic effects in a monolayer transition metal dichalcogenide semiconductor," Nat. Mater. 13, 1091 (2014).

[116] J.-W. van der Horst, P. A. Bobbert, M. A. J. Michels, G. Brocks, and P. J. Kelly, "Ab Initio calculation of the electronic and optical excitations in polythiophene: Effects of intra- and interchain screening," Phys. Rev. Lett. 83, 44134416 (1999).

[117] W. S. Leong, X. Luo, Y. Li, K. H. Khoo, S. Y. Quek, and J. T. L. Thong, "Low Resistance Metal Contacts to $\mathrm{MoS}_{2}$ Devices with Nickel-Etched-Graphene Electrodes," ACS Nano 9, 869-877 (2015). 
[118] Y. Du, L. Yang, J. Zhang, H. Liu, K. Majumdar, P. Kirsch, and P. Ye, “ $\mathrm{MoS}_{2}$ Field-Effect Transistors With Graphene/Metal Heterocontacts," Electron. Devic. Lett. 35, 599-601 (2014).

[119] H. Yuan, G. Cheng, L. You, H. Li, H. Zhu, W. Li, J. J. Kopanski, Y. S. Obeng, A. R. H. Walker, D. J. Gundlach, C. A. Richter, D. E. Ioannou, and Q. Li, “Influence of metal $\mathrm{MoS}_{2}$ interface on $\mathrm{MoS}_{2}$ transistor performance: Comparison of Ag and Ti contacts," ACS Appl. Mater. Interfaces 7, 1180-1187 (2015).

[120] H. Qiu, L. Pan, Z. Yao, J. Li, Y. Shi, and X. Wang, “Electrical characterization of back-gated bi-layer $\mathrm{MoS}_{2}$ field-effect transistors and the effect of ambient on their performances," Appl. Phys. Lett. 100, 123104 (2012).

[121] S. McDonnell, R. Addou, C. Buie, R. M. Wallace, and C. L. Hinkle, "Defectdominated doping and contact resistance in $\mathrm{MoS}_{2}$," ACS Nano 8, 2880-2888 (2014).

[122] M. Yankowitz, S. Larentis, K. Kim, J. Xue, D. McKenzie, S. Huang, M. Paggen, M. N. Ali, R. J. Cava, E. Tutuc, and B. J. LeRoy, "Intrinsic disorder in graphene on transition metal dichalcogenide heterostructures," Nano Lett. 15, 1925-1929 (2015).

[123] B. Radisavljevic, A. Radenovic, J. Brivio, V. Giacometti, and A. Kis, "Singlelayer $\mathrm{MoS}_{2}$ transistors," Nat. Nanotechnol. 6, 147-150 (2011).

[124] H. Wang, L. Yu, Y.-H. Lee, Y. Shi, A. Hsu, M. L. Chin, L.-J. Li, M. Dubey, J. Kong, and T. Palacios, "Integrated circuits based on bilayer $\mathrm{MoS}_{2}$ transistors," Nano Lett. 12, 4674-4680 (2012).

[125] D. Connelly, C. Faulkner, P. A. Clifton, and D. E. Grupp, "Fermi-level depinning for low-barrier Schottky source/drain transistors," Appl. Phys. Lett. 88, 012105 (2006).

[126] R. R. Lieten, S. Degroote, M. Kuijk, and G. Borghs, "Ohmic contact formation on n-type Ge," Appl. Phys. Lett. 92, 022106 (2008).

[127] I. Popov, G. Seifert, and D. Tománek, "Designing electrical contacts to $\mathrm{MoS}_{2}$ monolayers: A computational study," Phys. Rev. Lett. 108, 156802 (2012).

[128] M. Bokdam, P. A. Khomyakov, G. Brocks, Z. Zhong, and P. J. Kelly, "Electrostatic doping of graphene through ultrathin hexagonal boron nitride films," Nano Lett. 11, 4631-4635 (2011).

[129] R. Gillen, J. Robertson, and J. Maultzsch, "Indirect doping effects from impurities in $\mathrm{MoS}_{2}$ /h-BN heterostructures," Phys. Rev. B 90, 075437 (2014).

[130] O. Yazyev and A. Pasquarello, "Magnetoresistive junctions based on epitaxial graphene and hexagonal boron nitride," Phys. Rev. B 80, 035408 (2009). 
[131] D. Leuenberger, H. Yanagisawa, S. Roth, J. Osterwalder, and M. Hengsberger, "Disentanglement of electron dynamics and space-charge effects in time-resolved photoemission from $h$-BN/Ni(111)," Phys. Rev. B 84, 125107 (2011).

[132] Y. Cui, R. Xin, Z. Yu, Y. Pan, Z.-Y. Ong, X. Wei, J. Wang, H. Nan, Z. Ni, Y. Wu, T. Chen, Y. Shi, B. Wang, G. Zhang, Y.-W. Zhang, and X. Wang, "HighPerformance Monolayer $\mathrm{WS}_{2}$ Field-Effect Transistors on High- $\kappa$ Dielectrics," Adv. Mater. 27, 5230-5234 (2015).

[133] W. Zhang, J.-K. Huang, C.-H. Chen, Y.-H. Chang, Y.-J. Cheng, and L.-J. Li, "High-Gain Phototransistors Based on a CVD $\mathrm{MoS}_{2}$ Monolayer," Adv. Mater. 25, 3456-3461 (2013).

[134] Y.-H. Lee, X.-Q. Zhang, W. Zhang, M.-T. Chang, C.-T. Lin, K.-D. Chang, Y.-C. Yu, J. T.-W. Wang, C.-S. Chang, L.-J. Li, and T.-W. Lin, "Synthesis of LargeArea $\mathrm{MoS}_{2}$ Atomic Layers with Chemical Vapor Deposition," Adv. Mater. 24, 2320-2325 (2012).

[135] J. Chen, B. Liu, Y. Liu, W. Tang, C. T. Nai, L. Li, J. Zheng, L. Gao, Y. Zheng, H. S. Shin, H. Y. Jeong, and K. P. Loh, "Chemical vapor deposition of large-sized hexagonal WSe ${ }_{2}$ crystals on dielectric substrates," Adv. Mater. 27, 6722-6727 (2015).

[136] A. S. George, Z. Mutlu, R. Ionescu, R. J. Wu, J. S. Jeong, H. H. Bay, Y. Chai, K. A. Mkhoyan, M. Ozkan, and C. S. Ozkan, "Wafer scale synthesis and high resolution structural characterization of atomically thin $\mathrm{MoS}_{2}$ layers," Adv. Func. Mater. 24, 7461-7466 (2014).

[137] H. Fang, S. Chuang, T. C. Chang, K. Takei, T. Takahashi, and A. Javey, "Highperformance single layered $\mathrm{WSe}_{2}$ p-FETs with chemically doped contacts," Nano Lett. 12, 3788-3792 (2012).

[138] S. H. H. Shokouh, P. J. Jeon, A. Pezeshki, K. Choi, H. S. Lee, J. S. Kim, E. Y. Park, and S. Im, "High-Performance, Air-Stable, Top-Gate, p-Channel WSe ${ }_{2}$ Field-Effect Transistor with Fluoropolymer Buffer Layer," Adv. Func. Mater. 25, 7208-7214 (2015).

[139] S. Joonki, P. Tae-Eon, L. Der-Yuh, F. Deyi, P. Joonsuk, J. J. Hee, C. Yabin, K. Changhyun, J. Chaun, S. Yinghui, S. Robert, C. Joonyeon, T. Sefaattin, and W. Junqiao, "Doping against the native propensity of $\mathrm{MoS}_{2}$ : Degenerate hole doping by cation substitution," Nano Lett. 14, 6976-6982 (2014).

[140] D.-H. Kang, M.-S. Kim, J. Shim, J. Jeon, H.-Y. Park, W.-S. Jung, H.-Y. Yu, C.-H. Pang, S. Lee, and J.-H. Park, "High-performance transition metal dichalcogenide photodetectors enhanced by self-assembled monolayer doping," Adv. Func. Mater. 25, 4219-4227 (2015). 
[141] A. Allain and A. Kis, "Electron and hole mobilities in single-layer WSe ${ }_{2}$," ACS Nano 8, 7180-7185 (2014).

[142] D. Braga, I. G. Lezama, H. Berger, and A. F. Morpurgo, "Quantitative determination of the band gap of $\mathrm{WS}_{2}$ with ambipolar ionic liquid-gated transistors," Nano Lett. 12, 5218-5223 (2012).

[143] M. T. Greiner, L. Chai, M. G. Helander, W.-M. Tang, and Z.-H. Lu, "Metal/metal-oxide interfaces: How metal contacts affect the work function and band structure of $\mathrm{MoO}_{3}$," Adv. Func. Mater. 23, 215-226 (2013).

[144] M. Kröger, S. Hamwi, J. Meyer, T. Riedl, W. Kowalsky, and A. Kahn, "Role of the deep-lying electronic states of $\mathrm{MoO}_{3}$ in the enhancement of hole-injection in organic thin films," Appl. Phys. Lett. 95, 123301 (2009).

[145] S. Chuang, C. Battaglia, A. Azcatl, S. McDonnell, J. S. Kang, X. Yin, M. Tosun, R. Kapadia, H. Fang, R. M. Wallace, and A. Javey, "MoS 2 p-type Transistors and Diodes Enabled by High Work Function $\mathrm{MoO}_{x}$ Contacts," Nano Lett. 14, 1337-1342 (2014).

[146] S. McDonnell, R. Addou, C. Buie, R. M. Wallace, and C. L. Hinkle, "Defectdominated doping and contact resistance in $\mathrm{MoS}_{2}$," ACS Nano 8, 2880-2888 (2014).

[147] R. Kappera, D. Voiry, S. E. Yalcin, B. Branch, G. Gupta, A. D. Mohite, and M. Chhowalla, "Phase-engineered low-resistance contacts for ultrathin $\mathrm{MoS}_{2}$ transistors," Nat. Mat. 13, 1128-1134 (2014).

[148] K. Momma and F. Izumi, "VESTA: a three-dimensional visualization system for electronic and structural analysis," J. Appl. Cryst. 41, 653-658 (2008).

[149] H.-J. Chuang, X. Tan, N. J. Ghimire, M. M. Perera, B. Chamlagain, M. M.-C. Cheng, J. Yan, D. Mandrus, D. Tománek, and Z. Zhou, “High mobility WSe ${ }_{2}$ p- and n-type field-effect transistors contacted by highly doped graphene for low-resistance contacts," Nano Lett. 14, 3594-3601 (2014).

[150] W. S. Leong, X. Luo, Y. Li, K. H. Khoo, S. Y. Quek, and J. T. L. Thong, “Low resistance metal contacts to $\mathrm{MoS}_{2}$ devices with nickel-etched-graphene electrodes," ACS Nano 9, 869-877 (2015).

[151] L. F. Mattheiss, "Band structures of transition-metal-dichalcogenide layer compounds," Phys. Rev. B 8, 3719-3740 (1973).

[152] S.-L. Li, K. Komatsu, S. Nakaharai, Y.-F. Lin, M. Yamamoto, X. Duan, and K. Tsukagoshi, "Thickness Scaling Effect on Interfacial Barrier and Electrical Contact to Two-Dimensional MoS 2 Layers," ACS Nano 8, 12836-12842 (2014). 
[153] L. Britnell, R. V. Gorbachev, R. Jalil, B. D. Belle, F. Schedin, M. I. Katsnelson, L. Eaves, S. V. Morozov, A. S. Mayorov, N. M. R. Peres, A. H. C. Neto, J. Leist, A. K. Geim, L. A. Ponomarenko, and K. S. Novoselov, "Electron tunneling through ultrathin boron nitride crystalline barriers," Nano Lett. 12, 1707-1710 (2012).

[154] M. Farmanbar and G. Brocks, "First-principles study of van der Waals interactions and lattice mismatch at $\mathrm{MoS}_{2}$ /metal interfaces," Phys. Rev. B 93, 085304 (2016).

[155] T. Böker, R. Severin, A. Müller, C. Janowitz, R. Manzke, D. Voß, P. Krüger, A. Mazur, and J. Pollmann, "Band structure of $\mathrm{MoS}_{2}, \mathrm{MoSe}_{2}$, and $\alpha-\mathrm{MoTe}_{2}$ : Angle-resolved photoelectron spectroscopy and ab initio calculations," Phys. Rev. B 64, 235305 (2001).

[156] W. Schutte, J. D. Boer, and F. Jellinek, "Crystal structures of tungsten disulfide and diselenide," J. Solid State Chem. 70, 207 - 209 (1987).

[157] Y. Ohno, "Scanning tunneling microscopy of the misfit-layer compound $(\mathrm{SmS})_{1.19} \mathrm{NbS}_{2}$," Phys. Rev. B 58, 8042-8049 (1998).

[158] M.-H. Chiu, C. Zhang, H.-W. Shiu, C.-P. Chuu, C.-H. Chen, C.-Y. S. Chang, C.H. Chen, M.-Y. Chou, C.-K. Shih, and L.-J. Li, "Determination of band alignment in the single-layer $\mathrm{MoS}_{2} / \mathrm{WSe}_{2}$ heterojunction," Nat. Commun. 6, 7666 (2015).

[159] C. Ruppert, O. B. Aslan, and T. F. Heinz, "Optical Properties and Band Gap of Single- and Few-Layer MoTe 2 Crystals," Nano Lett. 14, 6231-6236 (2014).

[160] H. R. Gutiérrez, N. Perea-López, A. L. Elías, A. Berkdemir, B. Wang, R. Lv, F. López-Urías, V. H. Crespi, H. Terrones, and M. Terrones, "Extraordinary Room-Temperature Photoluminescence in Triangular $\mathrm{WS}_{2}$ Monolayers," Nano Lett. 13, 3447-3454 (2013).

[161] W. G. Dawson and D. W. Bullett, "Electronic structure and crystallography of $\mathrm{MoTe}_{2}$ and WTe $\mathrm{WT}_{2}$ " J. Phys. C: Solid State Phys. 20, 6159 (1987).

[162] Y. Zhang, T.-R. Chang, B. Zhou, Y.-T. Cui, H. Yan, Z. Liu, F. Schmitt, J. Lee, R. Moore, Y. Chen, H. Lin, H.-T. Jeng, S.-K. Mo, Z. Hussain, A. Bansil, and Z.$X$. Shen, "Direct observation of the transition from indirect to direct bandgap in atomically thin epitaxial $\mathrm{MoSe}_{2}, "$ Nat. Nanotechnol. 9, 111 (2014).

[163] J. Kang, S. Tongay, J. Zhou, J. Li, and J. Wu, "Band offsets and heterostructures of two-dimensional semiconductors," Appl. Phys. Lett. 102, 012111 (2013).

[164] A. K. Geim and K. S. Novoselov, “The rise of graphene,” Nat. Mater. 6, 183-191 (2007). 
[165] S. Helveg, J. V. Lauritsen, E. Lægsgaard, I. Stensgaard, J. K. Nørskov, B. S. Clausen, H. Topsøe, and F. Besenbacher, "Atomic-scale structure of singlelayer $\mathrm{MoS}_{2}$ nanoclusters," Phys. Rev. Lett. 84, 951-954 (2000).

[166] L. Ci, L. Song, C. Jin, D. Jariwala, D. Wu, Y. Li, A. Srivastava, Z. F. Wang, K. Storr, L. Balicas, F. Liu, and P. M. Ajayan, "Atomic layers of hybridized boron nitride and graphene domains," Nat. Mater. 9, 430-435 (2010).

[167] M. P. Levendorf, C.-J. Kim, L. Brown, P. Y. Huang, R. W. Havener, D. A. Muller, and J. Park, "Graphene and boron nitride lateral heterostructures for atomically thin circuitry," Nature 488, 627-632 (2012).

[168] A. M. van der Zande, P. Y. Huang, D. A. Chenet, T. C. Berkelbach, Y. You, G.-H. Lee, T. F. Heinz, D. R. Reichman, D. A. Muller, and J. C. Hone, "Grains and grain boundaries in highly crystallinemonolayer molybdenum disulphide," Nat. Mater. 12, 554-561 (2013).

[169] S. Najmaei, Z. Liu, W. Zhou, X. Zou, G. Shi, S. Lei, B. I. Yakobson, J.-C. Idrobo, P. M. Ajayan, and J. Lou, "Vapour phase growth and grain boundary structure of molybdenum disulphide atomic layers," Nat. Mater. 12, 754-759 (2013).

[170] H. Liu, L. Jiao, F. Yang, Y. Cai, X. Wu, W. Ho, C. Gao, J. Jia, N. Wang, H. Fan, W. Yao, and M. Xie, "Dense network of one-dimensional midgap metallic modes in monolayer $\mathrm{MoSe}_{2}$ and their spatial undulations," Phys. Rev. Lett. 113, 066105 (2014).

[171] M.-Y. Li, Y. Shi, C.-C. Cheng, L.-S. Lu, Y.-C. Lin, H.-L. Tang, M.-L. Tsai, C.-W. Chu, K.-H. Wei, J.-H. He, W.-H. Chang, K. Suenaga, and L.-J. Li, “Epitaxial growth of a monolayer $\mathrm{WSe}_{2}-\mathrm{MoS}_{2}$ lateral p-n junction with an atomically sharp interface," Science 349, 524-528 (2015).

[172] Y.-W. Son, M. L. Cohen, and S. G. Louie, "Half-metallic graphene nanoribbons," Nature 444, 347-349 (2006).

[173] O. V. Yazyev and M. I. Katsnelson, "Magnetic correlations at graphene edges: Basis for novel spintronics devices," Phys. Rev. Lett. 100, 047209 (2008).

[174] M. V. Bollinger, J. V. Lauritsen, K. W. Jacobsen, J. K. Nørskov, S. Helveg, and F. Besenbacher, "One-dimensional metallic edge states in $\mathrm{MoS}_{2}$," Phys. Rev. Lett. 87, 196803 (2001).

[175] M. V. Bollinger, K. W. Jacobsen, and J. K. Nørskov, “Atomic and electronic structure of $\mathrm{MoS}_{2}$ nanoparticles," Phys. Rev. B 67, 085410 (2003).

[176] A. Vojvodic, B. Hinnemann, and J. K. Nørskov, “Magnetic edge states in MoS $_{2}$ characterized using density-functional theory," Phys. Rev. B 80, 125416 (2009). 
[177] A. R. Botello-Méndez, F. López-Urías, M. Terrones, and H. Terrones, “Metallic and ferromagnetic edges in molybdenum disulfide nanoribbons," Nanotechnology 20, 325703 (2009).

[178] K. Andersen, K. W. Jacobsen, and K. S. Thygesen, "Plasmons on the edge of $\mathrm{MoS}_{2}$ nanostructures," Phys. Rev. B 90, 161410 (2014).

[179] X. Zou, Y. Liu, and B. I. Yakobson, "Predicting dislocations and grain boundaries in two-dimensional metal-disulfides from the first principles," Nano Lett. 13, 253-258 (2013).

[180] W. Zhou, X. Zou, S. Najmaei, Z. Liu, Y. Shi, J. Kong, J. Lou, P. M. Ajayan, B. I. Yakobson, and J.-C. Idrobo, "Intrinsic structural defects in monolayer molybdenum disulfide," Nano Lett. 13, 2615-2622 (2013).

[181] H. Schweiger, P. Raybaud, G. Kresse, and H. Toulhoat, "Shape and edge sites modifications of $\mathrm{MoS}_{2}$ catalytic nanoparticles induced by working conditions: A theoretical study," J. Catal. 207, 76 - 87 (2002).

[182] K. Nakada, M. Fujita, G. Dresselhaus, and M. S. Dresselhaus, "Edge state in graphene ribbons: Nanometer size effect and edge shape dependence," Phys. Rev. B 54, 17954-17961 (1996).

[183] A. R. Akhmerov and C. W. J. Beenakker, "Boundary conditions for dirac fermions on a terminated honeycomb lattice," Phys. Rev. B 77, 085423 (2008).

[184] P. Delplace, D. Ullmo, and G. Montambaux, "Zak phase and the existence of edge states in graphene," Phys. Rev. B 84, 195452 (2011).

[185] C. Ataca, H. Şahin, E. Aktürk, and S. Ciraci, "Mechanical and electronic properties of $\mathrm{MoS}_{2}$ nanoribbons and their defects," J. Phys. Chem. C 115, 3934-3941 (2011).

[186] Z. Wang, H. Li, Z. Liu, Z. Shi, J. Lu, K. Suenaga, S.-K. Joung, T. Okazaki, Z. Gu, J. Zhou, Z. Gao, G. Li, S. Sanvito, E. Wang, and S. Iijima, "Mixed Low-Dimensional Nanomaterial: 2D Ultranarrow $\mathrm{MoS}_{2}$ Inorganic Nanoribbons Encapsulated in Quasi-1D Carbon Nanotubes," J. Am. Chem. Soc. 132, 13840-13847 (2010).

[187] L. Kou, C. Tang, Y. Zhang, T. Heine, C. Chen, and T. Frauenheim, "Tuning magnetism and electronic phase transitions by strain and electric field in zigzag $\mathrm{MoS}_{2}$ nanoribbons," J. Phys. Chem. Lett. 3, 2934-2941 (2012).

[188] L. F. Seivane, H. Barron, S. Botti, M. A. Lopes Marques, Á. Rubio, and $\mathrm{X}$. López-Lozano, "Atomic and electronic properties of quasi-one-dimensional $\mathrm{MoS}_{2}$ nanowires," J. Mater. Res. 28, 240-249 (2013). 
[189] R.-L. Chu, G.-B. Liu, W. Yao, X. Xu, D. Xiao, and C. Zhang, "Spin-orbitcoupled quantum wires and majorana fermions on zigzag edges of monolayer transition-metal dichalcogenides," Phys. Rev. B 89, 155317 (2014).

[190] G. Xu, J. Wang, B. Yan, and X.-L. Qi, “Topological superconductivity at the edge of transition-metal dichalcogenides," Phys. Rev. B 90, 100505 (2014).

[191] M. C. Lucking, J. Bang, H. Terrones, Y.-Y. Sun, and S. Zhang, "Multivalencyinduced band gap opening at $\mathrm{MoS}_{2}$ edges," Chem. Mater. 27, 3326-3331 (2015).

[192] S. Pavlović and F. M. Peeters, "Electronic properties of triangular and hexagonal $\mathrm{MoS}_{2}$ quantum dots," Phys. Rev. B 91, 155410 (2015).

[193] J. Bernholc and S. T. Pantelides, "Scattering-theoretic method for defects in semiconductors. i. tight-binding description of vacancies in $\mathrm{Si}, \mathrm{Ge}$, and GaAs," Phys. Rev. B 18, 1780-1789 (1978).

[194] J. Pollmann and S. T. Pantelides, "Scattering-theoretic approach to the electronic structure of semiconductor surfaces: The (100) surface of tetrahedral semiconductors and $\mathrm{siO}_{2}, "$ Phys. Rev. B 18, 5524-5544 (1978).

[195] D. H. Lee and J. D. Joannopoulos, "Simple scheme for surface-band calculations. I," Phys. Rev. B 23, 4988-4996 (1981).

[196] D. H. Lee and J. D. Joannopoulos, "Simple scheme for surface-band calculations. II. The Green's function," Phys. Rev. B 23, 4997-5004 (1981).

[197] Y.-C. Chang and J. N. Schulman, "Complex band structures of crystalline solids: An eigenvalue method," Phys. Rev. B 25, 3975-3986 (1982).

[198] T. Ando, "Quantum point contacts in magnetic fields," Phys. Rev. B 44, 80178027 (1991).

[199] P. A. Khomyakov and G. Brocks, "Real-space finite-difference method for conductance calculations," Phys. Rev. B 70, 195402 (2004).

[200] P. A. Khomyakov, G. Brocks, V. Karpan, M. Zwierzycki, and P. J. Kelly, "Conductance calculations for quantum wires and interfaces: Mode matching and green's functions," Phys. Rev. B 72, 035450 (2005).

[201] P. A. Khomyakov and G. Brocks, "Stability of conductance oscillations in monatomic sodium wires," Phys. Rev. B 74, 165416 (2006).

[202] M. Zwierzycki, P. A. Khomyakov, A. A. Starikov, K. Xia, M. Talanana, P. X. Xu, V. M. Karpan, I. Marushchenko, I. Turek, G. E. W. Bauer, G. Brocks, and P. J. Kelly, "Calculating scattering matrices by wave function matching," Phys. Status. Solidi. B 245, 623-640 (2008). 
[203] F. Tisseur and K. Meerbergen, "The quadratic eigenvalue problem," SIAM Rev. 43, 235-286 (2001).

[204] G.-B. Liu, W.-Y. Shan, Y. Yao, W. Yao, and D. Xiao, "Three-band tight-binding model for monolayers of group-VIB transition metal dichalcogenides," Phys. Rev. B 88, 085433 (2013).

[205] H. Rostami, R. Roldán, E. Cappelluti, R. Asgari, and F. Guinea, “Theory of strain in single-layer transition metal dichalcogenides," Phys. Rev. B 92, 195402 (2015).

[206] Y. Hatsugai, "Chern number and edge states in the integer quantum hall effect," Phys. Rev. Lett. 71, 3697-3700 (1993).

[207] Y. Hatsugai, T. Fukui, and H. Aoki, "Topological analysis of the quantum hall effect in graphene: Dirac-fermi transition across van hove singularities and edge versus bulk quantum numbers," Phys. Rev. B 74, 205414 (2006).

[208] J. C. Y. Teo, L. Fu, and C. L. Kane, "Surface states and topological invariants in three-dimensional topological insulators: Application to $\mathrm{bi}_{1-x} \mathrm{sb}_{x}$," Phys. Rev. B 78, 045426 (2008).

[209] R. S. K. Mong and V. Shivamoggi, "Edge states and the bulk-boundary correspondence in dirac hamiltonians," Phys. Rev. B 83, 125109 (2011).

[210] G. Golub and C. F. van Loan, Matrix Computations (Johns Hopkins University Press, Baltimore, 1996).

[211] W. A. Harrison, "Tight-binding theory of surface states in metals," Phys. Scripta. 67, 253 (2003).

[212] X. Dang, J. D. Burton, A. Kalitsov, J. P. Velev, and E. Y. Tsymbal, “Complex band structure of topologically protected edge states," Phys. Rev. B 90, 155307 (2014).

[213] K. S. Yong, D. M. Otalvaro, I. Duchemin, M. Saeys, and C. Joachim, “Calculation of the conductance of a finite atomic line of sulfur vacancies created on a molybdenum disulfide surface," Phys. Rev. B 77, 205429 (2008).

[214] S. KC, R. C. Longo, R. Addou, R. M. Wallace, and K. Cho, "Impact of intrinsic atomic defects on the electronic structure of $\mathrm{MoS}_{2}$ monolayers," Nanotechnology 25, 375703 (2014).

[215] M. Gilbertini and N. Marzari, "Emergence of one-dimensional wires of free carriers in transition-metal-dichalcogenide nanostructures," Nano Lett. 15, 6229-6238 (2015). 
[216] R. Coehoorn, C. Haas, J. Dijkstra, C. J. F. Flipse, R. A. de Groot, and A. Wold, "Electronic structure of $\mathrm{MoSe}_{2}, \mathrm{MoS}_{2}$, and WSe 2 . I. Band-structure calculations and photoelectron spectroscopy," Phys. Rev. B 35, 6195-6202 (1987).

[217] S. S. Pershoguba and V. M. Yakovenko, "Shockley model description of surface states in topological insulators," Phys. Rev. B 86, 075304 (2012).

[218] T. F. Jaramillo, K. P. Jørgensen, J. Bonde, J. H. Nielsen, S. Horch, and I. Chorkendorff, "Identification of Active Edge Sites for Electrochemical $\mathrm{H}_{2}$ Evolution from $\mathrm{MoS}_{2}$ Nanocatalysts," Science 317, 100-102 (2007).

[219] C. Ataca and S. Ciraci, "Functionalization of single-layer $\mathrm{MoS}_{2}$ honeycomb structures," J. Phys. Chem. C 115, 13303-13311 (2011).

[220] T. Li and G. Galli, "Electronic properties of $\mathrm{MoS}_{2}$ nanoparticles," J. Phys. Chem. C 111, 16192-16196 (2007).

[221] K. Dolui, C. D. Pemmaraju, and S. Sanvito, "Electric field effects on armchair $\mathrm{MoS}_{2}$ nanoribbons," ACS Nano 6, 4823-4834 (2012).

[222] S. Helveg, J. V. Lauritsen, E. Lægsgaard, I. Stensgaard, J. K. Nørskov, B. S. Clausen, H. Topsøe, and F. Besenbacher, "Atomic-Scale Structure of SingleLayer $\mathrm{MoS}_{2}$ Nanoclusters," Phys. Rev. Lett. 84, 951-954 (2000).

[223] Y. Li, Z. Zhou, S. Zhang, and Z. Chen, "MoS 2 nanoribbons: High stability and unusual electronic and magnetic properties," J. Am. Chem. Soc. 130, 1673916744 (2008).

[224] M. Gilbertini, G. Pizzi, and N. Marzari, "Emergence of one-dimensional wires of free carriers in transition-metal-dichalcogenide nanostructures," Nat. Commun. 5, 5157 (2014).

[225] A. Castellanos-Gomez, R. Roldán, E. Cappelluti, M. Buscema, F. Guinea, H. S. J. van der Zant, and G. A. Steele, "Local Strain Engineering in Atomically Thin $\mathrm{MoS}_{2}$," Nano Lett. 13, 5361-5366 (2013).

[226] J. C. Slater and G. F. Koster, "Simplified LCAO Method for the Periodic Potential Problem," Phys. Rev. 94, 1498-1524 (1954). 


\section{Summary}

The discovery of graphene and its intriguing properties has given birth to the field of two-dimensional (2D) materials. These materials are characterized by a strong covalent bonding between the atoms within a plane, but weak, van der Waals, bonding between the planes. Such materials can be isolated as single or few atomic layers, or controllably grown by van der Waals epitaxy. Graphene has no band gap, but other 2D materials are natural semiconductors, such as the (group VI) transition metal dichalcogenides (TMDs) $\mathrm{MX}_{2}, \mathrm{M}=\mathrm{Mo}, \mathrm{W}, \mathrm{X}=\mathrm{S}, \mathrm{Se}, \mathrm{Te}$. As the band gap of single layers of these TMDs is direct, they attract a large interest because of their potential applications in opto-electronics.

This thesis concerns interfaces and edges of TMDs. It can be divided into two major parts. The first part, chapters 2,3, and 4, deals with the interfaces between these materials and metal contacts, which play a crucial role in the successful operation of electronic devices. In part two, chapters 5 and 6, we focus on the edges of 2D TMDs. Whereas the interior is semiconducting, many TMD edges are metallic and show a rich structure of electronic states with energies in the band gap. The metallicity is localized at the first few atomic rows along the edges, and it has one-dimensional character.

In chapter 1 we give a brief introduction to 2D materials and their edges, and to the physics of metal contacts. In this thesis we use density functional theory (DFT) calculations to study these systems. A short review of the basic ideas behind this formalism is given.

In chapter 2 we examine the interface properties of $\mathrm{MoS}_{2}$ contacts with a wide range of metals, where the interactions vary from weak physisorption to strong chemisorption. The $\mathrm{MoS}_{2}$ and metal surface lattices generally do not match at the interface. We examine the effects of lattice mismatch on the interface structure. Weak interface interactions require the inclusion of van der Waals forces. We critically compare results obtained with different density functionals. Somewhat surprisingly, even a weak interaction between $\mathrm{MoS}_{2}$ and a metal surface gives interface states that pin the Fermi level at an energy in the band gap. This generates an undesirable Schottky barrier at the interface.

In order to unpin the Fermi level, we insert a layer of $h$-BN between $\mathrm{MoS}_{2}$ and the metal substrates, as discussed in chapter 3. A monolayer of $h$-BN breaks the interac- 
tion between the metal surface and the overlayer and eliminates the interface states. In addition, adsorbing a $h$-BN layer on a metal surface effectively reduces its work function. This work function reduction leads to zero Schottky-barrier-height $n$-type contacts in $\mathrm{MoS}_{2}|h-\mathrm{BN}| \mathrm{Co}$ and $\mathrm{Ni}$ structures. A monolayer of $h$-BN is sufficiently thin, such that electrons can easily tunnel through this layer.

In chapter 4, we expand upon this idea and investigate possible buffer layers to make zero Schottky-barrier-height $p$-type contacts to $\mathrm{MX}_{2}$ semiconductors. We consider buffer layers such as graphene, a monolayer of $h-\mathrm{BN}$, or an oxide layer with a high electron affinity, such as $\mathrm{MoO}_{3}$. The most elegant solution is a metallic $\mathrm{M}^{\prime} \mathrm{X}_{2}^{\prime}$ buffer layer with a high work function. For example, a layer of $\mathrm{NbS}_{2}$ adsorbed on metal substrates gives high work function contacts with zero Schottky barriers for holes to all $\mathrm{MX}_{2}$ semiconductors.

One-dimensional edge states are studied in chapters 5 and 6 . In chapter 5 , we formulate a Green's function technique to model the edges of semi-infinite two dimensional systems. We express Green's functions in terms of Bloch matrices that can be constructed from the solutions of a quadratic eigenvalue equation. The technique can be applied to any localized basis representation of the Hamiltonian. Here we use it to calculate edge states of $\mathrm{MX}_{2}$ monolayers by means of tight-binding models. We begin with a three-bands tight-binding model, and study the basic zigzag and armchair edges, as well as edges with a more general orientation, structurally modifed edges, and grain boundaries. We extend the study to an eleven-band model comprising all valence orbitals of the $\mathrm{M}$ and $\mathrm{X}$ atoms.

In chapter 6, we analyze the electronic structure of edges of transition metal dichalcogenides (TMDs) in a nanoribbon geometry, by means of first-principles DFT calculations. We consider pristine edges in zigzag and armchair orientations, dressing the edges with additional chalcogen atoms, edge reconstructions, and possible magnetic orderings. 


\section{Samenvatting}

De ontdekking van grafeen met haar intrigerende eigenschappen heeft het tijdperk van twee-dimensionele (2D) materialen ingeluid. Deze materialen worden gekenschetst door sterke covalente bindingen tussen de atomen in een vlak, en zwakke vanderwaalsbindingen tussen de atoomvlakken. Ze kunnen geïsoleerd worden in de vorm van één of enkele atoomlagen, of gecontroleerd worden gegroeid door middel van vanderwaalsepitaxie. Grafeen heeft geen bandkloof, maar andere 2D materialen zijn natuurlijke halfgeleiders, zoals de (groep VI) overgangsmetaaldichalcogeniden (TMDs) $\mathrm{MX}_{2}, \mathrm{M}=\mathrm{Mo}, \mathrm{W}, \mathrm{X}=\mathrm{S}$, Se, Te. Omdat de bandkloof van een enkele laag direct is, trekken deze TMDs grote belangstelling voor mogelijke toepassingen in de optoëlectronica.

Dit proefschrift houdt zich bezig met grensvlakken en randen van TMDs. Het kan verdeeld worden in twee delen. Het eerste deel, met de hoofdstukken 2, 3 en 4 , gaat over grensvlakken tussen TMDs en metaalcontacten, welke een cruciale rol spelen in het functioneren van elektronische devices. In het tweede deel, met de hoofdstukken 5 en 6, richten we ons op de randen van 2D TMDs. Terwijl het binnenste van een TMD halfgeleidend is, is menige rand metallisch, en vertoont een rijke structuur aan elektronische toestanden met energieën in de bandkloof. Het metaalgedrag is gelokaliseerd in de eerste paar atoomrijen aan de randen, en het heeft een één-dimensioneel karakter.

In hoofdstuk 1 geven we een korte inleiding in 2D materialen en hun randen, en in de fysica van metaalcontacten. In dit proefschrift gebruiken we dichtheidsfunctionaaltheorieberekeningen om deze systemen te bestuderen. We geven een kort overzicht van de basisideeën achter dit formalisme.

In hoofdstuk 2 bestuderen we de grensvlakeigenschappen van $\mathrm{MoS}_{2}$-contacten met een uitgebreide selectie van metalen, waarbij de interacties variëren van zwakke fysisorptie tot sterke chemisorptie. De $\mathrm{MoS}_{2}$ - en de metaal-roosters zijn niet passend aan het grensvlak in het algemeen. We onderzoeken de effecten van deze roostermismatch op de grensvlakstructuur. Zwakke grensvlakinteracties vereisen het meenemen van vanderwaalskrachten. Resultaten verkregen met verschillende dichtheidsfunctionalen worden kritisch vergeleken. Enigszins verrast stellen we vast dat zelfs een zwakke interactie tussen $\mathrm{MoS}_{2}$ en een metaaloppervlak leidt tot grensvlaktoestanden die het ferminiveau vastpinnen op een energie in de bandkloof. Dit leidt tot 
een onwenselijke schottkybarrière aan het grensvlak.

Om het ferminiveau te ontpinnen voegen we een laag $h$-BN tussen $\mathrm{MoS}_{2}$ en het metaalsubstraat, hetgeen besproken wordt in hoofdstuk 3. Een $h$-BN monolaag verbreekt de interactie tussen het metaaloppervlak en de $\mathrm{MoS}_{2}$-laag, en elimineert de grensvlaktoestanden. Daarenboven wordt de werkfunctie van het metaaloppervlak gereduceerd door de adsorptie van een $h$-BNlaag. Deze werkfunctiereductie geeft $n$ type contacten in $\mathrm{MoS}_{2} \mid h$-BN|Co- en Ni-structuren met een schottkybarrièrehoogte nul. Een monolaag $h$-BN is voldoende dun, zodat electronen gemakkelijk door de laag kunnen tunnelen.

In hoofdstuk 4 breiden we dit idee uit, en onderzoeken mogelijke bufferlagen voor $p$-type contacten met $\mathrm{MX}_{2}$ halfgeleiders met een schottkybarrièrehoogte nul. We bekijken bufferlagen zoals grafeen, een monolaag $h$-BN, of een oxidelaag met een hoge elektronenaffiniteit gelijk $\mathrm{MoO}_{3}$. De meest elegante oplossing wordt gevormd door een metallische $\mathrm{M}^{\prime} \mathrm{X}_{2}^{\prime}$-bufferlaag met een hoge werkfunctie. Bijvoorbeeld, een laag $\mathrm{NbS}_{2}$ geadsorbeerd op een metaalsubstraat, geeft hoge werkfuncties, wat leidt tot contacten met alle $\mathrm{MX}_{2}$ halfgeleiders zonder schottkybarrières voor gaten.

Eén-dimensionele randtoestanden worden bestudeerd in de hoofdstukken 5 en 6 . In hoofdstuk 5 formuleren we een Greense functietechniek voor het modelleren van de randen van twee-dimensionele systemen. We drukken de Greense functies uit in blochmatrices die geconstrueerd worden uit de oplossingen van een kwadratische eigenwaardevergelijking. Deze techniek kan toegepast worden op iedere gelokaliseerde basisrepresentatie van de Hamiltoniaan. Hier gebruiken we haar om de randtoestanden van $\mathrm{MX}_{2}$ monolagen te berekenen met behulp van tight-bindingmodellen. We starten met een driebandstight-bindingmodel, en bestuderen de elementaire zigzagen leunstoelranden, alsmede randen met een meer algemene richting, randen met een gemodificeerde structuur, en korrelgrenzen. We breiden deze studie uit naar een elfbandsmodel dat alle valentie-orbitalen van de $\mathrm{M}$ en de $\mathrm{X}$ atomen bevat.

In hoofdstuk 6 analyseren we de elektronische toestanden van TMD randen in een nanolint-geometrie, met behulp van dichtheidsfunctionaaltheorieberekeningen. We beschouwen ongerepte randen in zigzag- en in leunstoeloriëntatie, randen aangekleed met toegevoegde chalcogenatomen, gereconstrueerde randen, en mogelijke magnetische ordeningen. 


\section{Acknowledgement}

Nederland is a 2D land with a convenient railway system. You can access to tiny towns easily by train. During the last year of my PhD, I travelled between Arnhem Presikhaf and Enschede back and forth and everyday, I saw different people at different stations hopping on and off the train. It reminded me meeting people at different stages of my PhD, where some of them stayed with me to the final stop and some changed their trains to some other directions. I am very grateful to all the people I have met through this long journey.

First of all, I would like to express my gratitude to Prof. Paul Kelly. Thank you for giving me this ticket to be a member of CMS group. It was really enjoyable having discussion over various topics in the coffee corner. Thanks to your wife, Andrea, helping my wife and hosting CMS gathering at your place.

My daily supervisor, Dr. Geert Brocks, thanks for all the interesting discussions over these years. I learned from you how to simplify everything, how to have a physical intuition to a problem, how to solve a problem, and obviously a lot more. I enjoyed the moments you came over my office and having our "koffie tijd" with a long discussion over history, languages, politics, middle east, and etc. On top of that, you helped me to improve my sense of humor. Indeed, this dissertation could not have been finished without your advices.

I would like to thank Prof. Peeters, Prof. van der Zaant, Prof. Caux, Prof. Koster, and Prof. Briels for being in my committee and taking the time to read this thesis.

I would like to take this opportunity to thank the CMS group for their support. Els, thank you for all the secretary and paper works upon my arrival up to leaving this group. Deniz, thanks for sharing all your knowledge about VASP and I wish you luck in your career in Belgium. Yi and Zhe, thank you for being a good neighbor and officemate for a while and wish you both success in Beijing. Menno, I learned from you analyzing the VASP output. Thanks for your help and wish you luck in your future career in Vienna. Diana, I have shared my office with you during the last two years of my PhD. Thank you for being a good friend and colleague and a good listener when I was stressed of finishing my thesis. I really enjoyed all the coffee times (especially Colombian Coffee) we had with Geert. You will defend your thesis soon and I wish you luck with your new life in Stuttgart. Kriti, there was a time where we were the only members left going for lunch. Thanks for the lunch times and cof- 
fee breaks that we shared. I am also grateful to the following members of CMS, for their various forms of support during my PhD- Anton, Zhicheng, Suleyman, Nirmal, Jayita, Elmer, and Rien.

Taher, we started this journey long time ago since when we were MSc students in SBU in Iran. We both applied for PhD positions in the CMS group, and got accepted here, and now we are about to finish our journey. I would never forget the moments we had from SBU ('Darake') in Iran to Witbreuksweg in Nederland. You are like my bro. Hadi, thanks for all the support and guidance through these years. I would not forget those days in your house with Marten and having barbeque. I always miss your favorite dish (Kebab). Damon, I still remember the first time I met you in the Dutch class, time flies buddy. From Disco Damon to Carre Hal B, spending all the times together with you, Taher, and Hadi was the most enjoyable part of my PhD. Mohammad, it was quite an adventures establishing IrNUT group at UT. It was, indeed, a hard work with you and Hamed writing statutes and translate it into Dutch. We have had lots of memories which I can not forget them. Thank you for motivating me to be more involved in different groups and thank you for being a true friend. I wish you luck in your new job.

Mitra, Sivash, Niloofar, Gerard, Ana, and Nazli, thank you for sharing your time with me. I remember those days, we lived in Matenweg and you guys were in BOX building. Having dinner during week days and weekends was great with you. Special thanks to Siavash and Mitra for their hospitality in Enschede, since we moved to Arnhem.

It was a great experience to meet many friends because of my activities in IrNUT like, Hamed, Sara, Davood, Navid, Saeed, Mozhde, Morteza, Hasan, Saeed, and Foad. My friends in Eindhoven, Saber, Hamed, Sama, Rahele, Hadi, and Shirin, thanks for hosting us whenever we were in Eindhoven. I would like to thank my football teammates in LatPers (Latin/Persian) football team. It was fun playing football with Hamed, Siavash, Mohammad, Jair, Ricardo, Jorge, Eduardo, Joao, Ruben, Chris, Mark, and Enrike. Thanks to Michiel, Wietske, Maartje, Emma, Kaulane and Ruben, you guys made my life enjoyable in Arnhem.

A special word of thanks also goes to my family for their continuous support and encouragement. My family has helped me stay strong through these difficult years. Their support and care helped me overcome setbacks and stay focused on my study. I greatly value their support and I deeply appreciate their belief in me.

At the end, I would like to thank my wife and friend, Sadaf. I am grateful for your love, encouragement, and tolerance, you made all the differences in my life. Without your patience and sacrifice, I could not have completed my PhD. You were all the time with me whenever I was down, disappointed, and stressed. You tried to motivate me and push me forward all the times. Especially, those days where we lived apart.

'Dit is de eindpunt van deze trein'. 


\section{Publications}

1. M. Farmanbar, T. Amlaki, and G. Brocks, "Green's function approach to edge states in transition metal dichalcogenides", submitted to Phys. Rev. B, see Chapter of 5 this thesis.

2. M. Farmanbar and G. Brocks, "Ohmic contacts to $2 \mathrm{D}$ semiconductors through van der Waals bonding", Adv. Electron. Mater., 1500405 (2016), see Chapter 4 of this thesis.

3. M. Farmanbar and G. Brocks, "First-principles study of van der Waals interactions and lattice mismatch at $\mathrm{MoS}_{2}$ /metal interfaces", Phys. Rev. B 93, 085304 (2016), see Chapter 2 of this thesis.

4. A. Acun, L. Zhang, P. Bampoulis, M Farmanbar, A. van Houselt, A. N. Rudenko, M. Lingenfelder, G. Brocks, B. Poelsema, M. I. Katsnelson, H. J. W. Zandvliet, "Germanene: the germanium analogue of graphene", J. Phys.: Condens. Matter 27, 443002 (2015).

5. M. Farmanbar and G. Brocks, "Controlling the Schotkky barrier at $\mathrm{MoS}_{2} / \mathrm{metal}$ contacts by inserting a BN monolayer", Phys. Rev. B 91, 161304(R), 2015, see Chapter 3 of this thesis.

6. R. Heimbuch, A. van Houselt, M. Farmanbar, G. Brocks, H. J. W. Zandvliet, "Interfering Bloch waves in a 1D electron system", J. Phys.: Condens. Matter 25, 014014 (2013). 


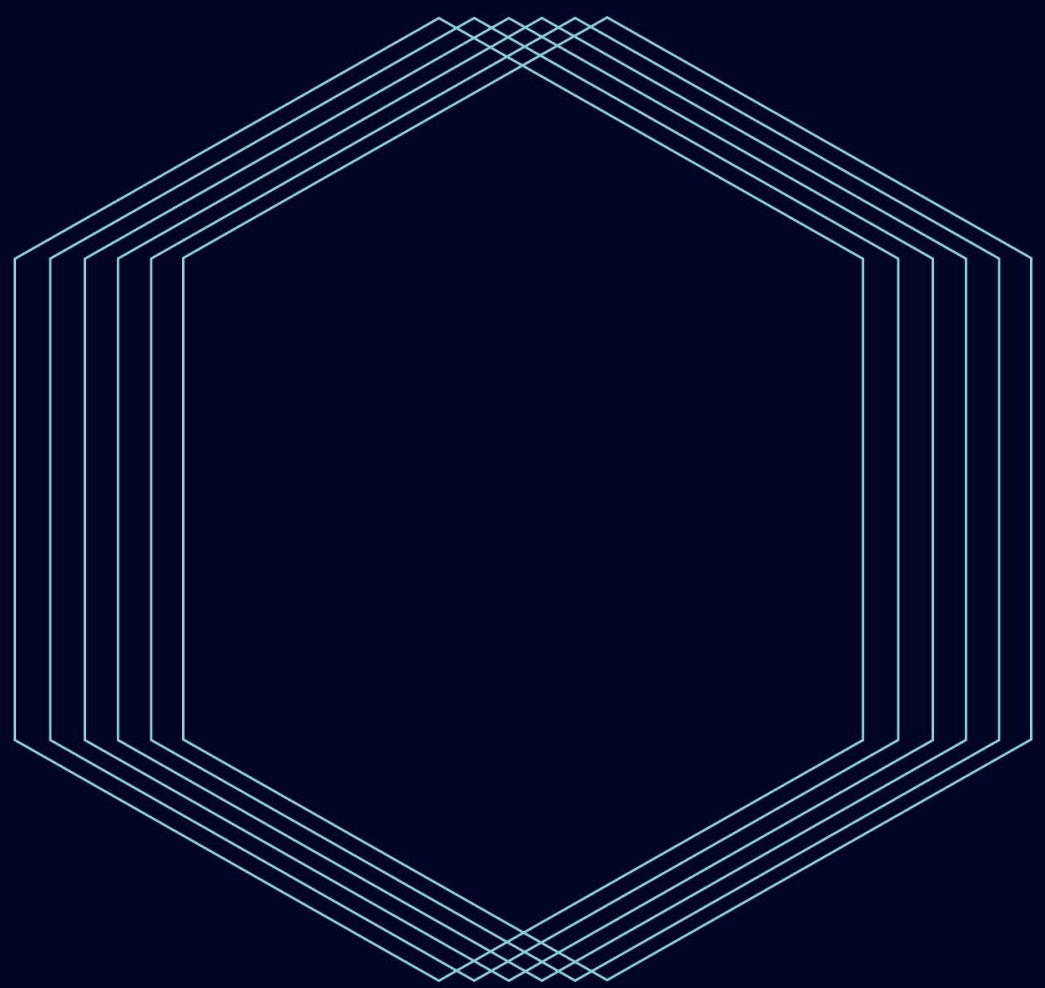

"Everything should be made as simple as possible, but not simpler."

Albert Einstein 